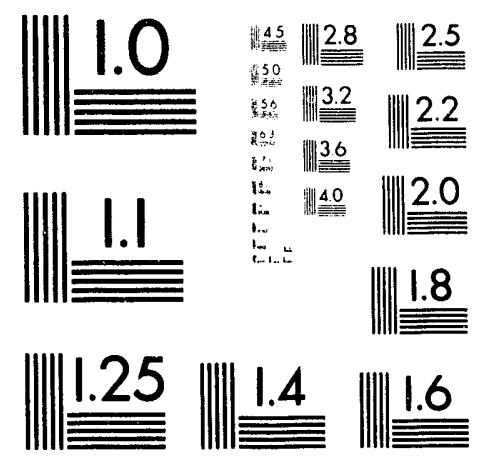



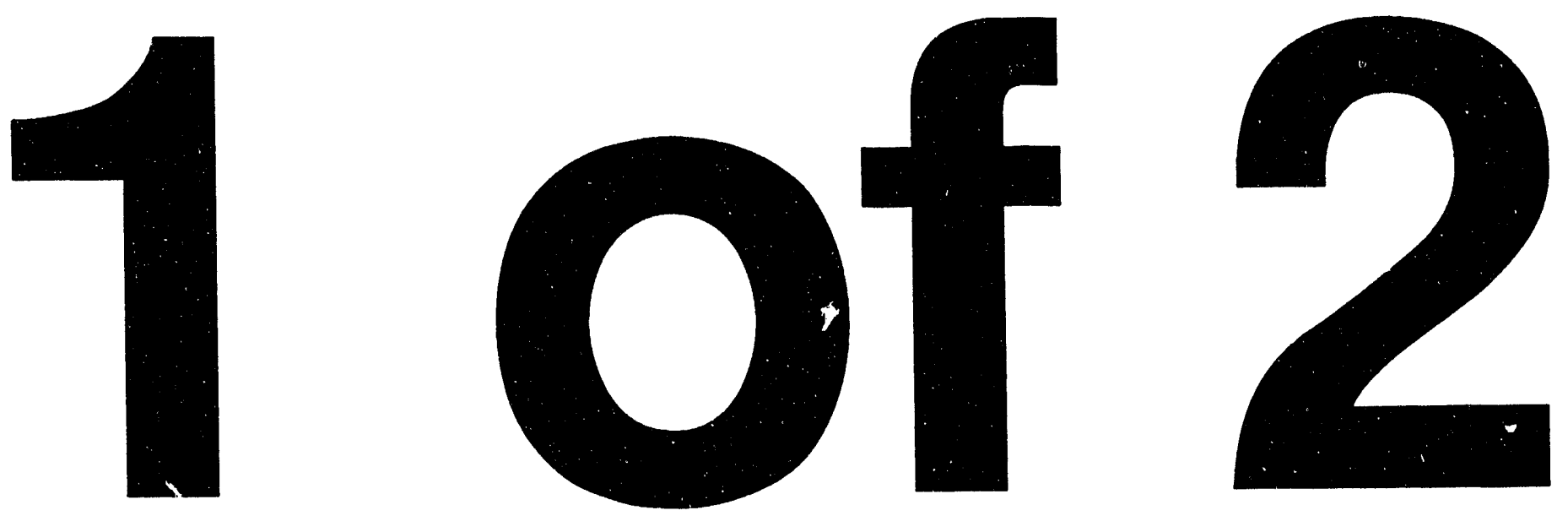
An Investigation of the Impact of Inorganic Air Pollutants on Soils in Saguaro National Monument, Tucson, Arizona

Ernest S. Gladney

Roger W. Ferenbaugh

Kenneth W. Stolte*

Daniel M. Duriscoe** 


\title{
AN INVESTIGATION OF THE IMPACT OF INORGANIC AIR POLLUTANTS ON SOILS IN SAGUARO NATIONAL MONUMENT, TUCSON, ARIZONA
}

by

\author{
Ernest S. Gladney \\ Roger W. Ferenbaugh \\ Kenneth W. Stolte \\ Daniel M. Duriscoe
}

\begin{abstract}
Environmental data related to the evaluation of inorganic air pollution input to the Saguaro National Monument ecosystem were collected over four years. The data specific to soils are presented in this document. The enrichment factor approach is employed to provide a framework for simplified interpretation of this large collection of data.
\end{abstract}

\section{INTRODUCTION}

Under the Clean Air Act, Federal Land Managers, including the National Park Service (NPS), have direct responsibility for managing certain designated federal lands to actively protect their "air quality related values" [Section 165(d)(2)]. The NPS Air Quality Division is concerned about the potential impact of trace element emissions from various sources on resources of national parks and monuments throughout the Southwest.

Saguaro National Monument (SAGU) was created by Executive Proclamation in 1933, in response to concerns for preservation of stands of saguaro cacti in the vicinity of Tucson, Arizona. Nearly 45 years passed before settlement of boul.dary disputes and acquisition of land resulted in the establishment of the final boundaries shown in Fig. 1 (1). In the meantime, in the early 1940s, observations were made that older saguaro cacti in the Monument were dying and, furthermore, that reestablishment of saguaro seedlings was not occurring.

After the origiral observation in 1940 that saguaro cacti were dying, the :0-called "saguaro decline" was initially attributed to bacterial infection. During the winter of 1941-1942, large numbers of presumably diseased cacti were removed and buried in an effort to stop the spread of the alleged infection (1). Subsequently, references to saguaro decline dating as far back as 1896 were discovered, and the prevailing viewpoint of the condition changed to regard it as a naturallyoccurring cyclical fluctuation resulting from general overmaturity of the saguaro population. 
With this change in viewpoint, the concern with respect to the declining saguaro population broadened to include failure of seedling reestablishment as well as death of older individuals. Various factors investigated as potential influences on seedling reestablishment included cattle grazing (1), nutritional deficiencies, and changes in other edaphic facto:s (2)

Although some effects on saguaro populations and seedling reestablishment could be linked to climatic conditions and cattle grazing, no satisfactory overall explanation of the continuing saguaro decline has been forthcoming. Studies of saguaro populations in other locations indicate that some populations are flourishing (3), reinforcing the conclusion that the decline of the population at SAGU is somewhat of a local phenomenon. This continuing decline is, however, real, and it is documented in a series of photographs taken from the same vantage point in 1935, 1965, and 1985. The extreme thinning of the cactus forest is obvious and dramatic.

More recently, air pollution has become suspect as a potential causal factor in the continuing saguaro decline. Air pollution sources in the form of copper smelters throughout southern Arizona date back to the late 19th century. Although emissions from copper smelters have fluctuated with economic conditions, the Tucson metropolitan area represents a continuouslygrowing source of urban air pollution. The combined emissions from these sources include particulate matter containing metals and other inorganic compounds, sulfur dioxide, and a variety of organic compounds. Aircraft application of agricultural chemicals in the vicinity of Tucson represents another airborne source of potentially toxic organic compounds.

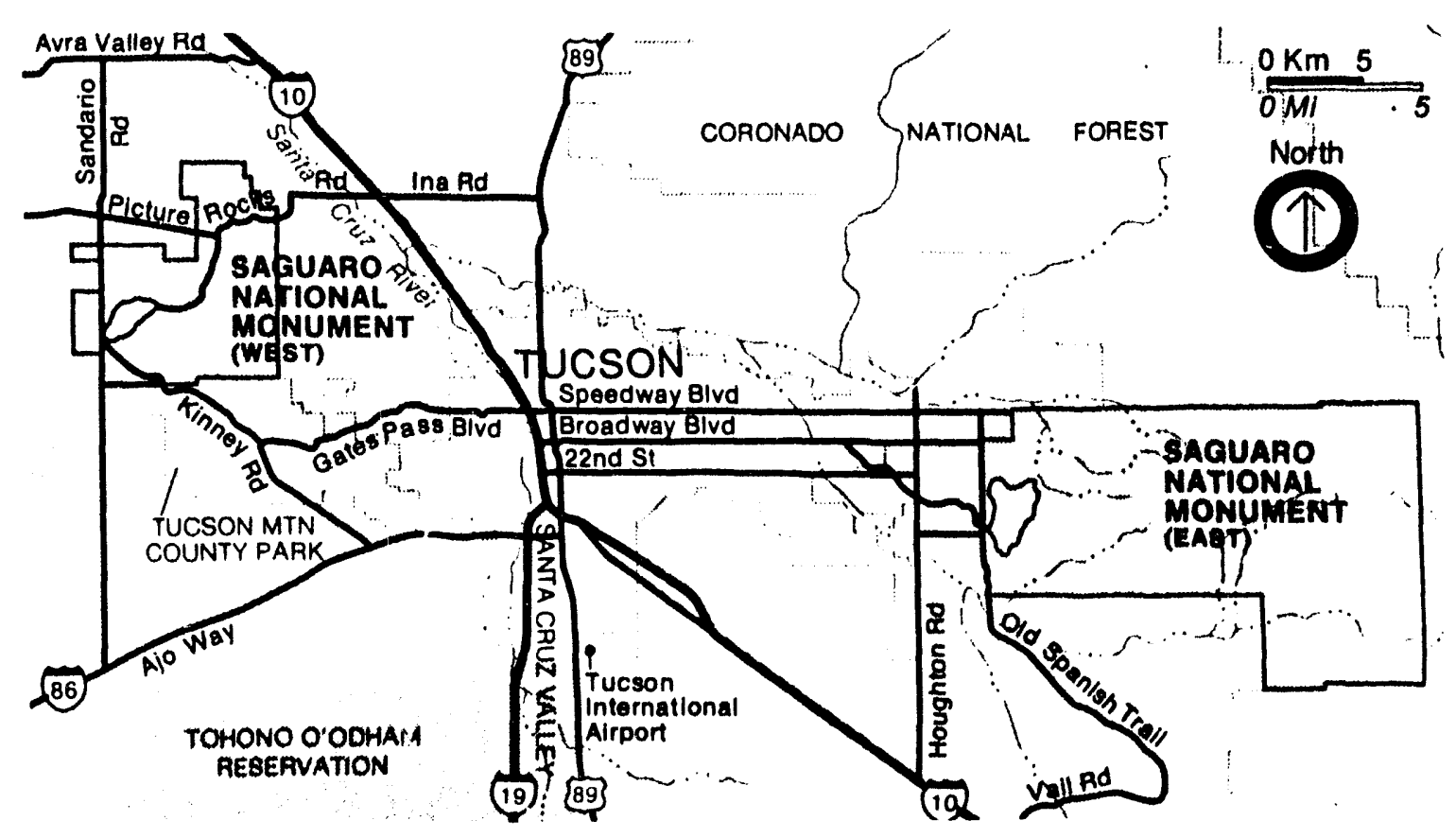

Figure 1. Saguaro National Monument, Arizona. 
Another change in the biological resource of the Monument has been recently documented. Graybill and Rose (4), at the Laboratory of Tree Ring Research at the University of Arizona, have observed extreme growth declines in number of individuals in the Ponderosa and White Pine populations above 6500 feet elevation in the Rincon Mountain District while conducting a longrange study of growth trends in pines, in Arizona. Air pollution is suspected as possibly playing a major role. Stolte (5) established a number of control plots in the same areas to monitor ozone injury to vegetation.

In 1987, a preliminary survey of soils and selected vegetation was undertaken at SAGU under the auspices of an Interagency Agreement between the NPS and the Los Alamos National Laboratory (LANL). The purpose of this survey was an attempt to identify chemical profiles that might indicate air pollution impact upon the Monument. These efforts were expanded in subsequent years, with the last sampling taking place in early 1990. An overview of sampling site locations is shown in Fig. 2 and the detailed locations are given in Appendix A. Samples of soil were collected inside and outside the Monument (including soil at several distances from a nearby smelter), from within the drip line at the base of four species of trees in the Catalina and Rincon Mountains along the northern and eastern boundaries of the Rincon Mountain District (RMD) of

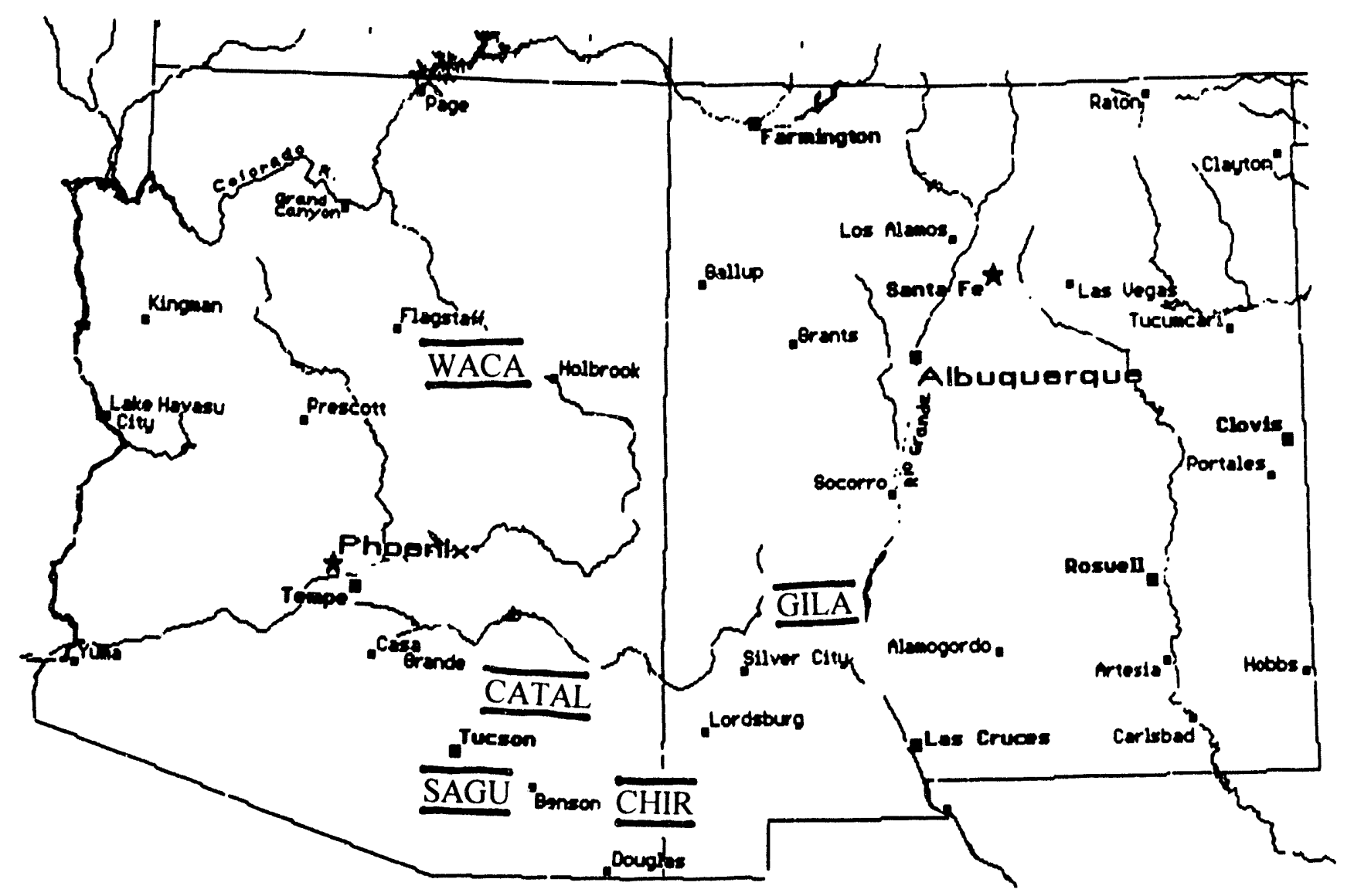

Figure 2. Overview of regional sampling sites. 
SAGU, where tree grouth decline is being obscrved, and from near the bases of numbers of both healthy and browned saguaro cacti. Only trees that had been cored as part of the Graybill and Rose study (4) were selected for soil sampling at their bases. Regional control samples of soils were collected from Walnut Canyon and Chiricahua National Monuments in Arizona, and Gila Cliffs National Monument in New Mexico from within the drip line at the base of Ponderosa pines included in the Graybill and Rose study (4). This report presents the chemical composition data that have been collected on these soils

\section{METHODS}

Surface soil samples (0-2 cm depth) were collected using sampling tools made of either polyechylene or Teflon. Deeper samples were taken using standard U.S. Forest Service soil coring tools made of stainless steel. The soil samples were collected in polyethylene bags and returned to Los Alamos for chemical analysis. Samples were air-dried for several days and then sieved through a standard 20-mesh brass screen to remove organic debris and larger rock fragments. The soils from the Tucson Mountain District (TMD) were further separated into four size fractions $(20,60,100$, and $<100$ mesh) using stacked standard brass screens. The sieved samples were stored in polyethylene bottles prior to analysis.

To date, most elemental analysis has been conducted via instrumental neutron activation analysis at the LANL Omega West Reactor by the authors and the staff of the reactor facility. These methods are described in detail in References 6 and 7. Up to 45 elements have been quantitatively determined. A small set of these samples has been analyzed for fluorine using ion selective electrode techniques. Carbonate carbon has been measured in some of the soils via acidevolution gravimetry, and both bound and unbound water have been determined by coulometry in the same subset of soils. These methods are described in detail in Gautier and Gladney (8). All chemical analyses have been quality assured by concurrent analysis of National Institute of Standards and Technology (formerly National Bureau of Standards), U. S. Geological Survey, and Canadian Geological Survey reference materials according to our established policies (9). All quality control/quality assurance data are presented in Appendix B.

Two approaches have proven especially valuable in attempting to study the impact of atmospheric particulates on various air sheds: 1) source characterization of emissions focusing on the elemental composition as a function of particle size; and 2) the determination of enrichment factors (EF) of trace elements in particulates, vegetation, ard soils to suggest elements which may show impact from atmospheric input. In general, one can divide atmospheric particulate sources into two categories: natural background and anthropogenic. Natural sources of particulates include continental weathering products (soils, rocks), marine aerosols, volcanic materials injected during eruptions, and meteoritic debris ablated during atmospheric entry (10-12). Anthropogenic sources may be large "point-sources" such as smelters, specialized industries (microelectronics), energy conversion facilities (electric generation, coal gasification, etc.), and waste incineration (13-15). They may also be widespread, diffuse sources such as internal combustion engines, agricultural operations, or aircraft (16). 
Dilution of soil samples with varying amounts of organic matter may give the appearance of wide differences in elemental concentration. Similarly, changes in total atmospheric particulate loading can mask potential source patterns These variations can be eliminated by dividing the absolute concentrations of elements of interest by that of a reference element that is abundant in the atmospheric aerosol, or common in the soils, or easily measured by the analytical method of choice with a high degree of precision and accuracy.

One can obtain even more useful information by performing a second normalization to the elemental abundance pattern that has been established for individual sources of airborne materials Enrichment factors can be calculated using the following equation:

$$
E F=\frac{[X] a /[R] a}{[X] c /[R] c}
$$

where $[X] \mathrm{a}=$ concentration of element $\mathrm{X}$ in atmospheric aerosol or soil

$[R] a=$ concentration of reference element $R$ in atmospheric aerosol or soil

$[X] c=$ concentration of element $X$ in average crustal material, and

$[R] c=$ conceritration of reference element $R$ in average crustal material

The reference element for this calculation must be chosen with some care. It is important that the one chosen have only one source in the atmosphere or soil, be fairly abundant, and/or easily measured with high precision and accuracy. The usual reference elements are $\mathrm{Si}, \mathrm{Al}$, and $\mathrm{Sc}$ for the soils cemponent; $\mathrm{Na}$ and $\mathrm{Cl}$ for the marine component; and $\mathrm{Pb}$ and $\mathrm{Br}$ for the automotive component. If neutron activation analysis is used as the analytical methodology, $\mathrm{Si}$ is eliminated since it is difficult to detect using this technique. More detailed discussion of the enrichment factor concept can be found in References 17-19.

Much suspended particulate matter is soil and rock dust injected by wind erosion. Relative concentrations of elements borne by such dust are probably the same as those of the major element used in the double normalization. Also, relative concentrations of many elements in several source materials are similar to those of crustal material. For example, the alumino-silicate portion of coal (ash) has a concentration pattern much like that of average crustal material. Thus, when an element has a large EF after the double normalization, one must look to special sources to find explanations for its anomalous concentration.

If the airborne or soil materials are primarily from the source from which the reference element is selected, the EF values should be close to unity. For elements that display EF values strongly in excess of 1 , the implication is that there are one or more additional sources that contribute significantly to the concentration of those elements. We have chosen the EF range of $0.5-2.5$ to 
represent the "no impact" level. Since natural variability in soils can be quite high, one must be careful not to over interpret EF data near 1.0, but rather lonk for large deviations, preferably an order of magnitude or more.

\section{RESIUTS AND DISCUSSION}

Over 825 soil samples from the various sites have been analyzed for up to 48 constituents. All individual concentration data are presented in Appendix C. The EF approach, detailed in Ref. 19 , has been used in an attempt to summarize this mass of data for more incisive interpretation and eventual resource management use. All EFs were computed relative to Wedepohl's average elemental abundance in the earth's crust (20). Overall means and standard deviations of these EFs are presented in Table 1 as a function of sampling site and depth. While all elements were measured at all sites, certain constituents were consistently below our limits of detection and could not be used to calculate EFs. This is shown as "---" if all samples were below detection limits, and as a mean without a standard deviation where only one or two samples yielded EFs.

Several elements ( $\mathrm{Ag}, \mathrm{As}, \mathrm{Br}, \mathrm{Ga}, \mathrm{In}, \mathrm{Pb}, \mathrm{Sb}, \mathrm{Se}, \mathrm{V}$, and $\mathrm{Zn}$ ) have become established as indicators of various types of sources for regional inorganic air pollution (19). Silver and $\mathrm{Ga}$ are often associated with waste incineration; $\mathrm{As}, \mathrm{Sb}, \mathrm{Se}$, and $\mathrm{V}$ with electric power production; In with metal smelter emissions; and $\mathrm{Br}, \mathrm{Pb}$, and $\mathrm{Zn}$ with transportation. This list is worth keeping in mind during the following discussion on interpretation of the data gathered at SAGU.

Our soil data in Table I can be broken down into roughly four groups for the purposes of this presentation: 1) elements which are so often at or below our limits of detection that no valid conclusions may be drawn; 2) elements which show depletions relative to Wedepohl's crustal abundances, with EFs consistently below $0.5 ; 3)$ those which exhibit EFs near $1.0(0.5-2.5)$; and 4) constituents which have EFs greater than 2.5 for a significant number of sites. This last group should be examined for evidence of anthropogenic air pollution input to the SAGU ecosystem. The reason we have chosen the EF range of $0.5-2.5$ to represent "no impact" for this approach is that natural variability in soils can be quite high. One must be careful not to over interpret EF data near 1.0, but instead be looking for large deviations, preferably an order of magnitude or more. Even elevated levels in soils may not be biologically available, and plants growing in these soils need to be analyzed in addition to the soils to provide complete pathway analysis.

The data in Table I indicate that $\mathrm{Ag}, \mathrm{I}, \mathrm{In}$, and Se are candidates for the first group; i.e. uninterpretable because of limited data. There are some data for $\mathrm{In}$ and Se with above detection limit concentrations, and these will be discussed along with the other indicator elements, although any conclusions for these two elements have high levels of uncertainty. Several elements which fall into the second category (depleted) seldom exhibit this behavior elsewhere, in our experience. Calcium, $\mathrm{Cl}, \mathrm{Co}, \mathrm{Mg}$, and sometimes $\mathrm{Na}$ and $\mathrm{Sc}$ show depletions over large areas of SAGU and in many of the background sites as well. Although this has no air pollution implication, it seems to represent an unanticipated regisnal characteristic of southwestern soils. This also provides a counter argument to the hypothesis that the saguaro cacti are suffering from elevated levels of 
作 Table 1. Sumary of Enrichment Factors for Soils Collected at Saguaro Nat lonal Monument, Catalina Mountains, Chirlcahua National Monument, Walnut Canyon National Monument, and Gila Cliffs Natlonal Monument, 1907 - 1990

\section{San Manuel Smelter}

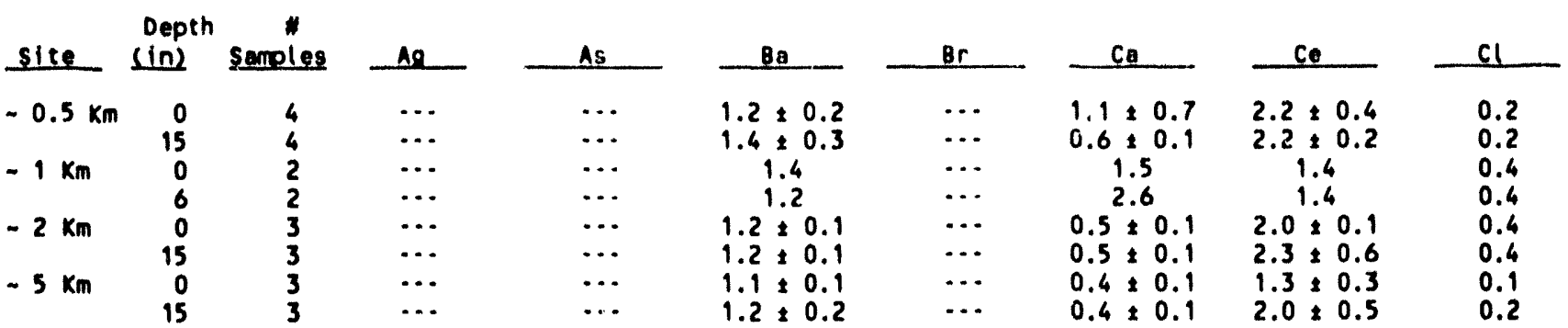

Saquare Mational Monument

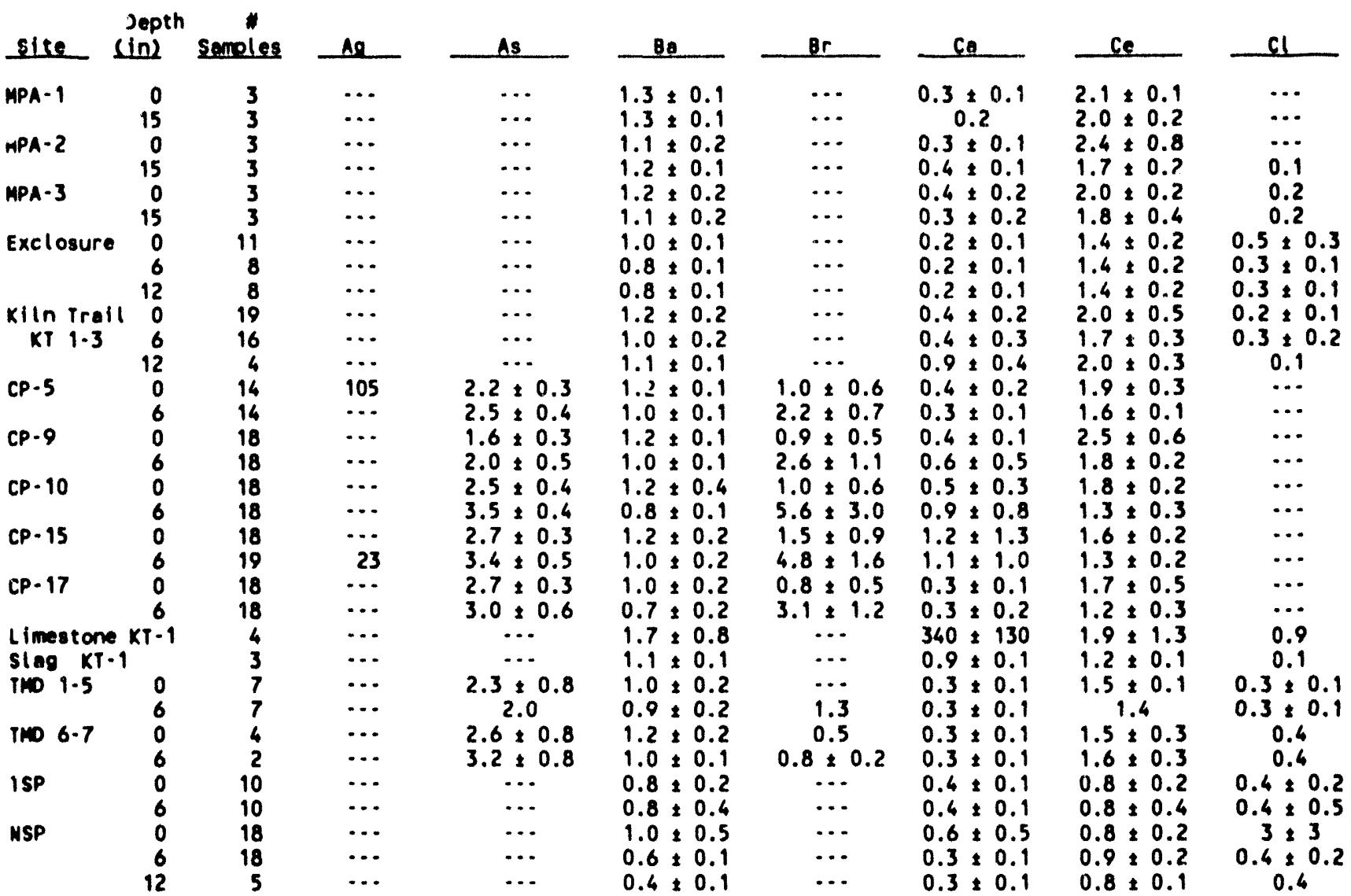

Regional Backeround

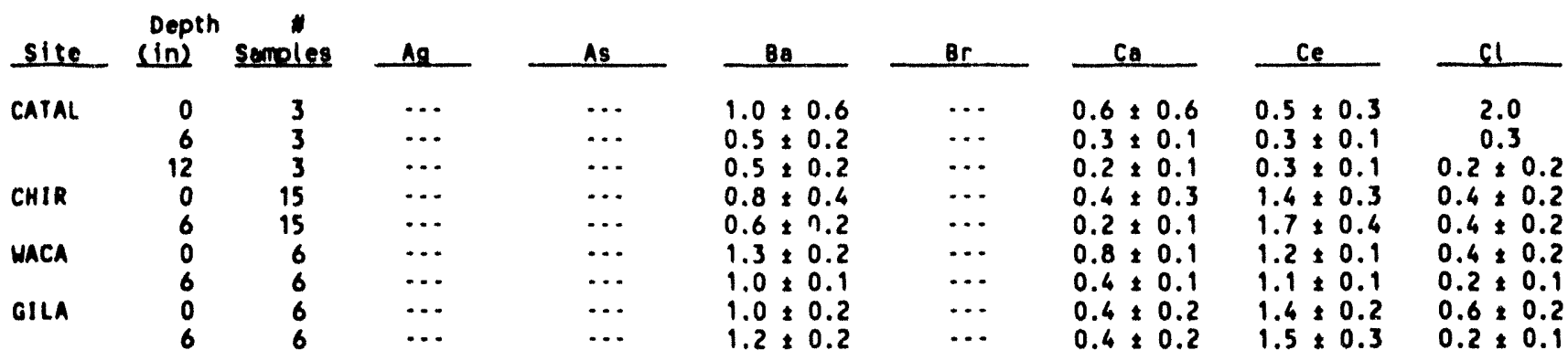


Table L. Sumary of Enrichment factors for Solls Collaceded of Saguaro Mational Monument Cataline Mountaing Chiricahua National Monument. Walnut Canyon National Monument, and Gila Cliffs National Monument, 1987. 1990 (cont.)

San Manuel Smelter

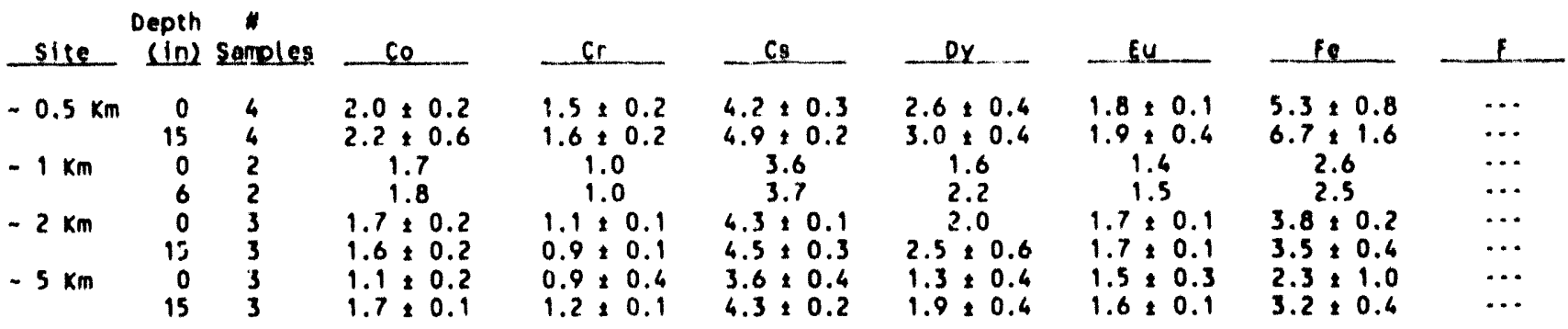

Saquare National Monument

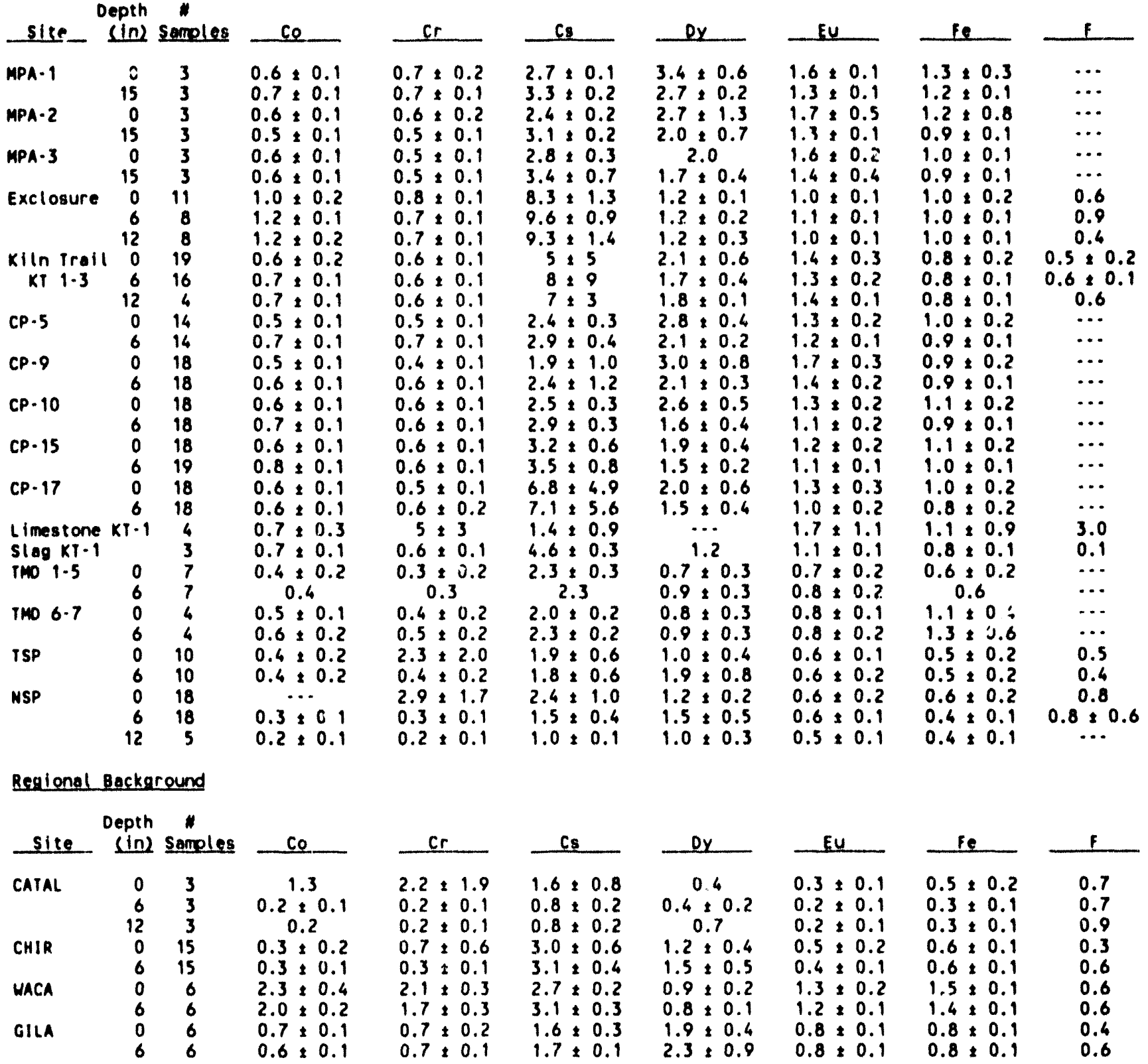




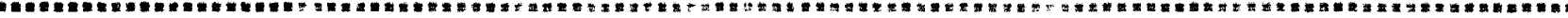

Toble 1. Sumary of Enrichment factore for Solls Colleced at Sagusro Mational Momment, Catalina Mountains, Chirlcahue Mational Monument, Walrut Canyon National Monument, and Gila Cliffs Matlonal Monument, 1987 . 1990 (cont.)

Sen Honuel smelter

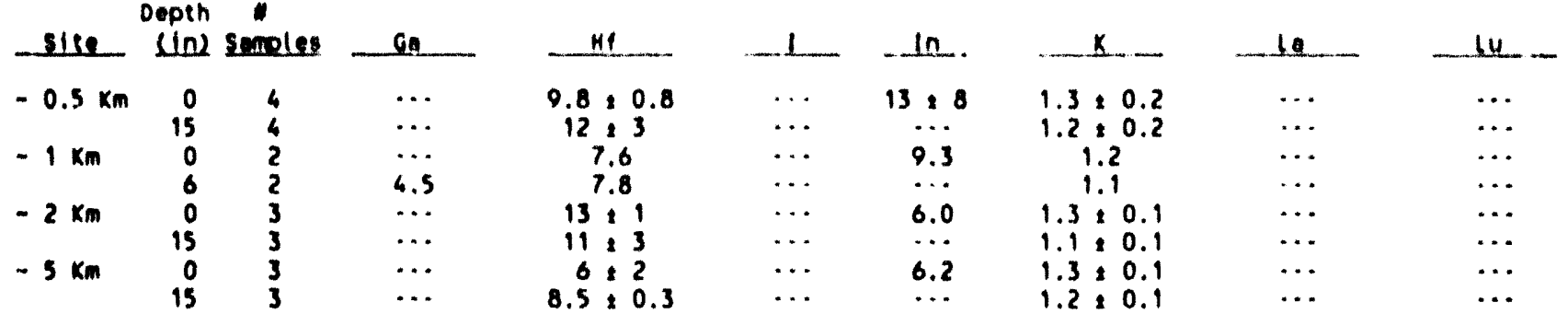

sesure Netional Homment

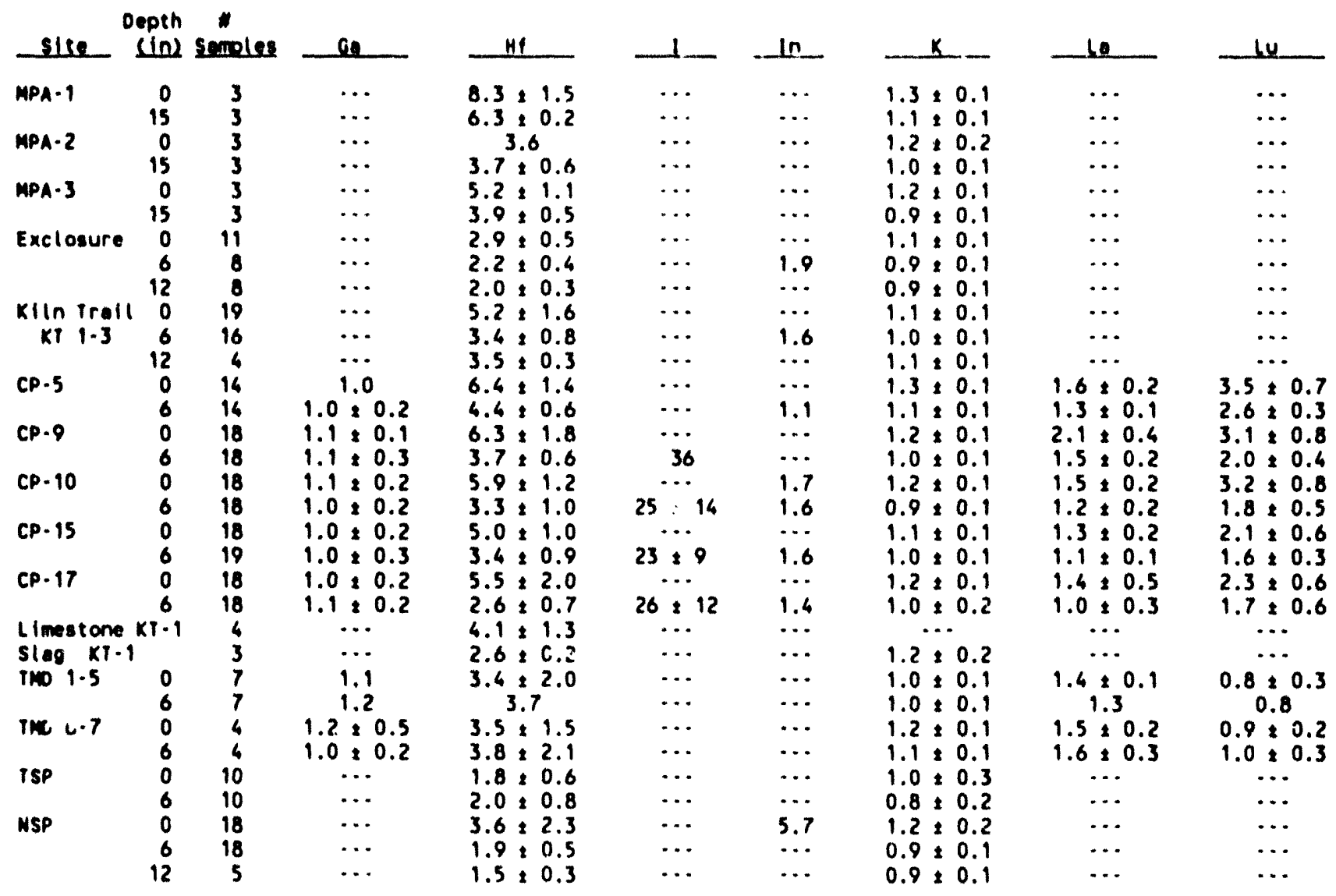

Begionel Beckgroud

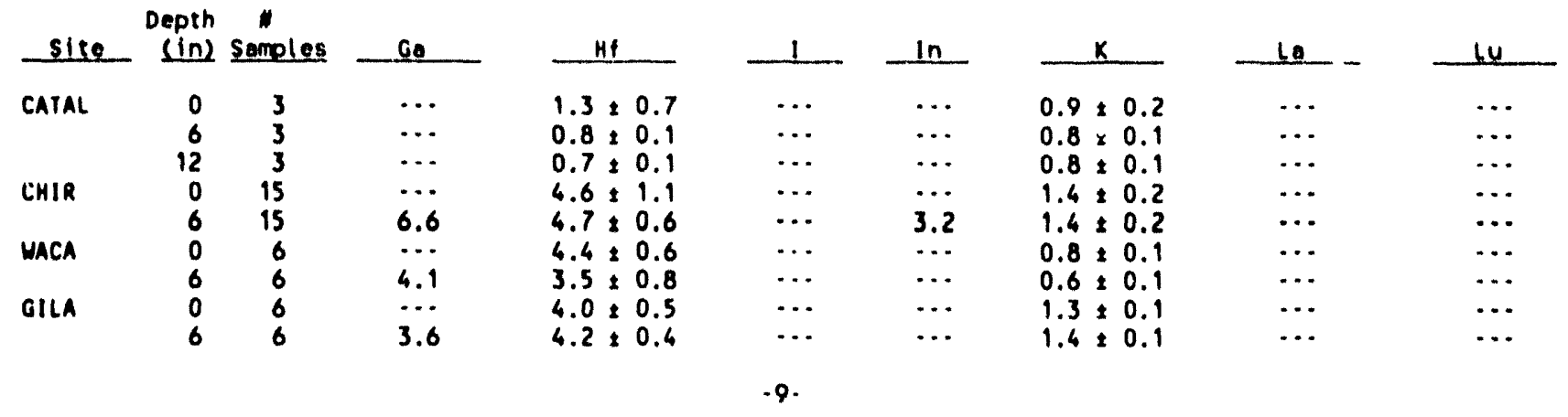




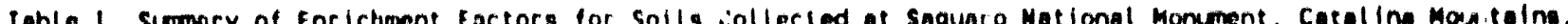

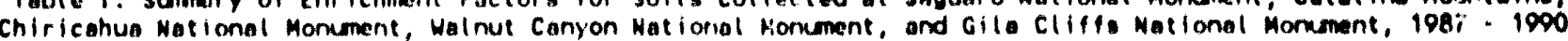
(cont.)

San Manuel Smelter

\begin{tabular}{|c|c|c|c|c|c|c|c|c|c|}
\hline slte & (n) & semples & $\mathrm{Mg}$ & An & $\mathrm{Na}$ & Nd & Rb & sh & S6 - \\
\hline $\begin{array}{l}-0.5 \mathrm{~km} \\
-1 \mathrm{~km}\end{array}$ & $\begin{array}{r}6 \\
15 \\
0 \\
6\end{array}$ & $\begin{array}{l}4 \\
4 \\
2 \\
2\end{array}$ & $\begin{array}{l}0.7 \\
0.7 \\
0.8 \\
0.9\end{array}$ & $\begin{array}{c}4.6: 1.4 \\
5.6: 1.1 \\
2.2 \\
2.0\end{array}$ & $\begin{array}{c}0.7+0.2 \\
0.7 \div 0.1 \\
0.6 \\
0.5\end{array}$ & $\begin{array}{l}\cdots \\
\cdots \\
\cdots \\
\cdots\end{array}$ & $\begin{array}{c}2.2 \neq 0.3 \\
1.9: 0.1 \\
1.4 \\
1.2\end{array}$ & $\begin{array}{c}34: 14 \\
14 \div 4 \\
36 \\
13\end{array}$ & $\begin{array}{c}1.8 * 1.2 \\
2.3 \times 1.5 \\
1.2 \\
1.0\end{array}$ \\
\hline $\begin{array}{l}-2 \mathrm{~km} \\
-5 \mathrm{~km}\end{array}$ & $\begin{array}{r}0 \\
15 \\
0 \\
15\end{array}$ & $\begin{array}{l}3 \\
3 \\
3 \\
3\end{array}$ & $\begin{array}{l}0.6 \\
0.4 \\
0.4 \\
0.5\end{array}$ & $\begin{array}{c}3.3: 0.4 \\
3.7: 0.4 \\
2.4 \\
2.5: 0.4\end{array}$ & $\begin{array}{l}0.8: 0.1 \\
0.7: 0.1 \\
0.7: 0.1 \\
0.6: 0.1\end{array}$ & $\begin{array}{l}\cdots \\
\cdots \\
\cdots \\
\cdots\end{array}$ & $\begin{array}{l}2.1 * 0.2 \\
1.7: 0.2 \\
2.0 * 0.3 \\
1.6 * 0.1\end{array}$ & $\begin{array}{rll}22 & : 4 \\
10.5 & : 0.3 \\
20 & : 7 \\
13.9 & : 1.3\end{array}$ & $\begin{array}{l}1.6: 0.1 \\
1.6: 0.2 \\
1.1: 0.3 \\
1.6=0.1\end{array}$ \\
\hline
\end{tabular}

sequare Netionel Menument

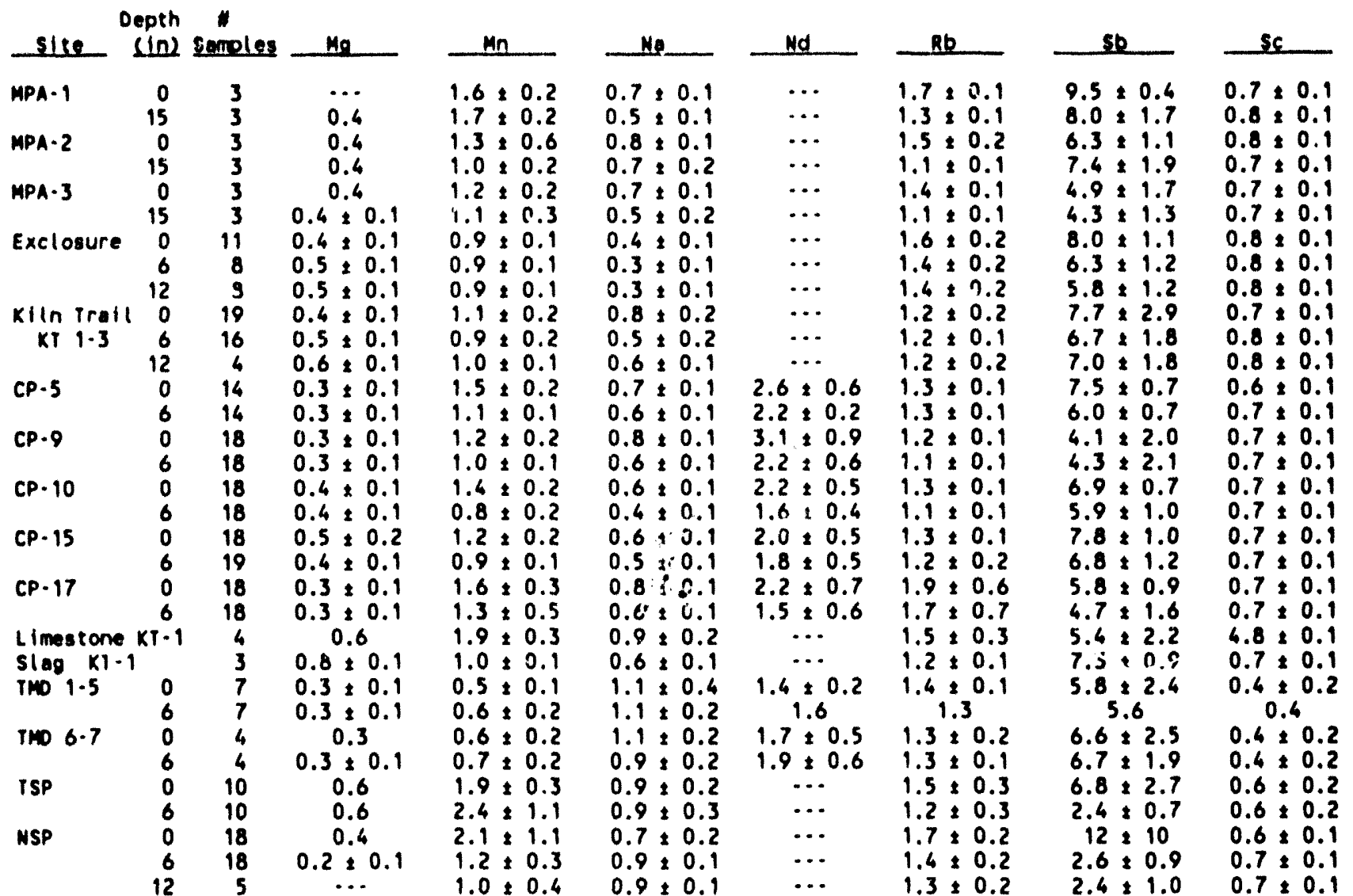

Regional Background

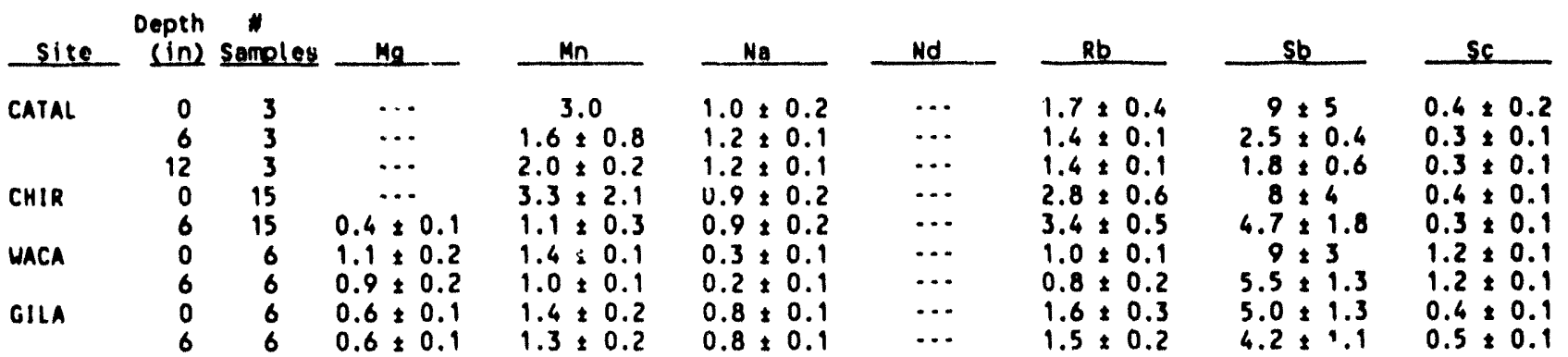




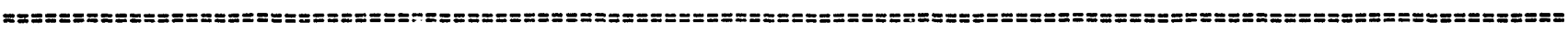

Table 1. Summary of Enrichment Factors for Soils Collected at Saguaro National Monument, Catalina Mountains, Chiricahua National Monument, Walnut Canyon National Monument, and Gila Cliffs National Monument, 1987 - 1990 (cont.)

San Manuel Smelter

\begin{tabular}{|c|c|c|c|c|c|c|c|c|c|}
\hline site & $\begin{array}{r}\text { Depth } \\
\text { (in) }\end{array}$ & $\begin{array}{c}\# \\
\text { Samples }\end{array}$ & Se & $\mathrm{si}$ & $\mathrm{Sm}$ & $\mathrm{Sr}$ & Ta & Tb & Th \\
\hline $\begin{array}{l}-0.5 \mathrm{~km} \\
-1 \mathrm{~km}\end{array}$ & $\begin{array}{r}0 \\
15 \\
0 \\
6\end{array}$ & $\begin{array}{l}4 \\
4 \\
2 \\
2\end{array}$ & $\begin{array}{l}\ldots \\
\ldots \\
\ldots \\
\ldots\end{array}$ & $\begin{array}{c}1.2 \pm 0.1 \\
1.2 \pm 0.2 \\
1.2 \\
1.1\end{array}$ & $\begin{array}{c}2.4 \pm 0.1 \\
3.0 \pm 0.7 \\
1.3 \\
2.2\end{array}$ & $\begin{array}{l}\cdots \\
\cdots \\
\cdots\end{array}$ & $\begin{array}{c}1.8 \pm 0.4 \\
2.8 \pm 0.9 \\
0.9 \\
1.0\end{array}$ & $\begin{array}{l}\cdots \\
1.0 \\
1.6\end{array}$ & $\begin{array}{c}3.2 \pm 0.6 \\
4.4 \pm 2.2 \\
2.2 \\
1.8\end{array}$ \\
\hline $\begin{array}{l}-2 \mathrm{~km} \\
-5 \mathrm{~km}\end{array}$ & $\begin{array}{r}0 \\
15 \\
0 \\
15\end{array}$ & $\begin{array}{l}3 \\
3 \\
3 \\
3\end{array}$ & $\begin{array}{l}\cdots \\
\ldots \\
\cdots \\
\cdots\end{array}$ & $\begin{array}{l}1.2 \pm 0.1 \\
1.2 \pm 0.1 \\
1.3 \pm 0.1 \\
1.2 \pm 0.1\end{array}$ & $\begin{array}{l}2.1 \pm 0.1 \\
2.4 \pm 0.3 \\
1.6 \pm 0.4 \\
2.0 \pm 0.2\end{array}$ & 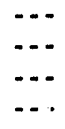 & $\begin{array}{l}1.7 \pm 0.2 \\
1.7 \pm 0.2 \\
1.1 \pm 0.3 \\
1.3 \pm 0.4\end{array}$ & $\begin{array}{l}\cdots \\
\cdots \\
\cdots\end{array}$ & $\begin{array}{l}3.7 \pm 1.6 \\
3.0 \pm 0.1 \\
2.2 \pm 1.5 \\
2.1 \pm 0.5\end{array}$ \\
\hline
\end{tabular}

Saguaro National Monument

\begin{tabular}{|c|c|c|c|c|c|}
\hline Site & $\begin{array}{l}\text { epth } \\
\text { (in) }\end{array}$ & $\begin{array}{c}\# \\
\text { Samples }\end{array}$ & Se & Si & $\mathrm{Sm}$ \\
\hline \multirow[t]{2}{*}{ MPA-1 } & 0 & 3 & $\cdots$ & $1.4 \pm 0.1$ & $2.4 \pm 0.4$ \\
\hline & 15 & 3 & $\cdots$ & $1.1 \pm 0.1$ & $1.9 \pm 0.3$ \\
\hline \multirow[t]{2}{*}{ MPA-2 } & 0 & 3 & $\cdots$ & $1.2 \pm 0.1$ & $2.0 \pm 0.7$ \\
\hline & 15 & 3 & $\cdots$ & $1.1 \pm 0.1$ & $1.8 \pm 0.3$ \\
\hline \multirow[t]{2}{*}{ MPA-3 } & 0 & 3 & $\cdots$ & $1.2 \pm 0.1$ & $2.3 \pm 0.2$ \\
\hline & 15 & 3 & $\cdots$ & $0.9 \pm 0.1$ & $2.1 \pm 0.3$ \\
\hline \multirow[t]{3}{*}{ Exclosure } & 0 & 11 & $\cdots$ & $0.9 \pm 0.1$ & $1.2 \pm 0.2$ \\
\hline & 6 & 8 & $\cdots$ & $0.8 \pm 0.1$ & $1.3 \pm 0.2$ \\
\hline & 12 & 8 & $\cdots$ & $0.7 \pm 0.1$ & $1.2 \pm 0.2$ \\
\hline \multirow{3}{*}{$\begin{array}{c}\text { Kiln Trail } \\
\text { KT } 1-3\end{array}$} & 10 & 19 & $\cdots$ & $1.1 \pm 0.1$ & $1.9 \pm 0.5$ \\
\hline & 6 & 16 & $\cdots$ & $0.9 \pm 0.2$ & $1.6 \pm 0.2$ \\
\hline & 12 & 4 & $\cdots$ & $1.0 \pm 0$. & $1.8 \pm 0.3$ \\
\hline \multirow{2}{*}{$C P-5$} & 0 & $\because$ & 9.8 & $\cdots$ & $2.1 \pm 0.3$ \\
\hline & 6 & 4 & 7.7 & $\cdots$ & $1.6 \pm 0.1$ \\
\hline \multirow[t]{2}{*}{$C P-9$} & 0 & 18 & $\cdots$ & $\cdots$ & $2.7 \pm 0.6$ \\
\hline & 6 & 18 & $\cdots$ & $\cdots$ & $1.9 \pm 0.3$ \\
\hline \multirow[t]{2}{*}{$C P-10$} & 0 & 18 & $\cdots$ & $\cdots$ & $1.9 \pm 0.2$ \\
\hline & 6 & 18 & $\cdots$ & $\cdots$ & $1.4 \pm 0.3$ \\
\hline \multirow[t]{2}{*}{ CP- 15} & 0 & 18 & $\cdots$ & $\cdots$ & $1.6 \pm 0.2$ \\
\hline & 6 & 19 & $\cdots$ & $\cdots$ & $1.4 \pm 0.2$ \\
\hline \multirow[t]{2}{*}{ CP- 17} & 0 & 18 & 8.8 & $\cdots$ & $1.8 \pm 0.6$ \\
\hline & 6 & 18 & $\cdots$ & $\cdots$ & $1.4 \pm 0.4$ \\
\hline \multicolumn{2}{|c|}{$\begin{array}{l}\text { Limestone } \mathrm{KT}-1 \\
\mathrm{Sl} \\
\mathrm{KT}-1\end{array}$} & 4 & 0.1 & $\cdots$ & $1.1 \pm 0.5$ \\
\hline \multirow{2}{*}{\multicolumn{2}{|c|}{$\begin{array}{l}\text { Slag } K T-1 \\
\text { TMD } 1-5\end{array}$}} & 3 & $\cdots$ & $1.0 \pm 0.1$ & $1.2 \pm 0.1$ \\
\hline & & 7 & 7.3 & $\cdots$ & $1.0 \pm 0.2$ \\
\hline TMD 6-7 & 6 & 7 & $\cdots$ & $\cdots$ & 0.9 \\
\hline TMD 6-7 & $\begin{array}{l}0 \\
6\end{array}$ & $\begin{array}{l}4 \\
4\end{array}$ & $\begin{array}{l}200 \\
280\end{array}$ & $\cdots$ & $\begin{array}{l}1.1 \pm 0.3 \\
1.1 \pm 0.4\end{array}$ \\
\hline \multirow[t]{2}{*}{ TSP } & 0 & 10 & $\cdots$ & $1.0 \pm 0.1$ & $1.2-0.5$ \\
\hline & 6 & 10 & $\cdots$ & $0.9 \pm 0.2$ & $1.1 \pm 0.5$ \\
\hline \multirow[t]{2}{*}{ NSP } & 0 & 18 & $\cdots$ & $1.1 \pm 0.3$ & $0.8 \pm 0.2$ \\
\hline & $\begin{array}{r}6 \\
12\end{array}$ & $\begin{array}{r}18 \\
5\end{array}$ & $\cdots$ & $\begin{array}{l}0.8 \pm 0.1 \\
0.7 \pm 0.1\end{array}$ & $\begin{array}{l}0.8 \pm 0 . ? \\
0.8 \pm 0.2\end{array}$ \\
\hline
\end{tabular}

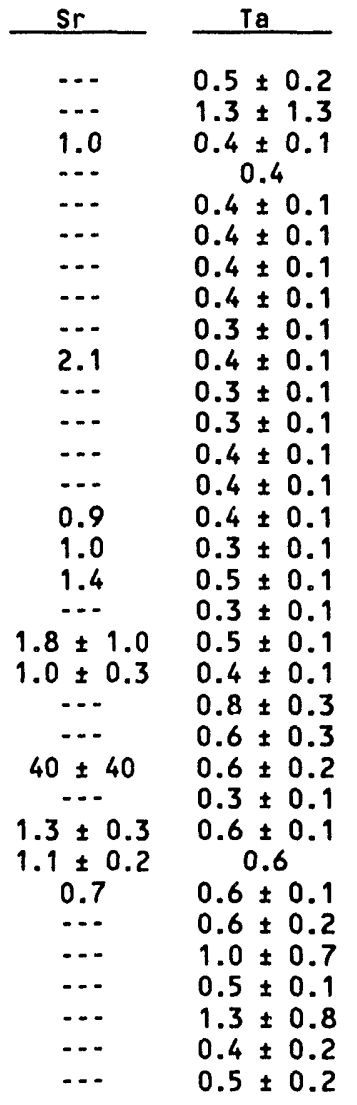

\begin{tabular}{|c|c|}
\hline Tb & Th \\
\hline$\cdots$ & $2.9 \pm 0.2$ \\
\hline$\cdots$ & $2.4 \pm 0.3$ \\
\hline$\cdots$ & $2.8 \pm 1.0$ \\
\hline$\cdots$ & $2.0 \pm 0$ \\
\hline$\cdots$ & $2.4 \pm 0.4$ \\
\hline$\cdots$ & $2.0 \pm$ \\
\hline $0.7 \pm 0.1$ & $1.6 \pm 0.2$ \\
\hline $0.7 \pm 0.1$ & $1.5 \pm 0.2$ \\
\hline $0.7 \pm 0.2$ & $1.4 \pm 0.2$ \\
\hline $\begin{array}{l}1.4 \pm 0.5 \\
1.2 \pm 0.2\end{array}$ & $\begin{array}{l}2.5 \pm 0.7 \\
1.9 \pm 0.2\end{array}$ \\
\hline $2 \pm 0.1$ & $1.9 \pm 0$ \\
\hline $6 \pm 0.2$ & $2.4 \pm 0$ \\
\hline $1.4 \pm 0.1$ & $2.0 \pm$ \\
\hline $2.0 \pm 0.5$ & $3.0 \pm 0.8$ \\
\hline $1.6 \pm 0.3$ & $2.3 \pm 0$ \\
\hline $1 \pm 0.2$ & \pm \\
\hline $1.2 \pm 0.3$ & \pm \\
\hline $1.0 \pm 0.2$ & \pm 0.2 \\
\hline $1.3 \pm 0.4$ & $2.1 \pm 0.7$ \\
\hline $\begin{array}{l}0.9 \pm 0.2 \\
1.7 \pm 0.7\end{array}$ & $\begin{array}{l}1.5 \pm 0 . \\
0.8 \pm 0 .\end{array}$ \\
\hline \pm 0 & $1.4 \pm 0$ \\
\hline $\begin{array}{c}5 \pm 0.2 \\
0.4\end{array}$ & $2.9 \pm 1$. \\
\hline $.6 \pm 0.2$ & $3.4 \pm 2.3$ \\
\hline $0.6 \pm 0.2$ & $3.6 \pm 1$ \\
\hline $\begin{array}{l}1.1 \pm 0.5 \\
1.1 \pm 0.4\end{array}$ & $\begin{array}{l}1.1 \pm 0 . \\
1.0 \pm 0 .\end{array}$ \\
\hline$\cdots$ & $1 \pm$ \\
\hline- & $3 \pm 0.3$ \\
\hline$\ldots$ & + \\
\hline
\end{tabular}

Regional Background

\begin{tabular}{|c|c|c|c|c|c|c|c|c|c|}
\hline Site & $\begin{array}{r}\text { Depth } \\
\text { (in) }\end{array}$ & $\begin{array}{c}\# \\
\text { Samples } \\
\end{array}$ & Se & si & $\mathrm{sm}$ & $\mathrm{Sr}$ & Ta & Tb & Th \\
\hline CATAL & 0 & 3 & ... & $1.0 \pm 0.1$ & $0.7 \pm 0.4$ & $\cdots$ & $0.9 \pm 0.5$ & $\cdots$ & $0.6 \pm 0.3$ \\
\hline & 6 & 3 & $\cdots$ & $0.9 \pm 0.1$ & $0.4 \pm 0.1$ & $\ldots$ & $0.5 \pm 0.1$ & $\cdots$ & $0.4 \pm 0.1$ \\
\hline & 12 & 3 & -- & $0.9 \pm 0.1$ & $0.4 \pm 0.1$ & $\cdots$ & $0.5 \pm 0.1$ & $\cdots$ & $0.5 \pm 0.1$ \\
\hline CHIR & 0 & 15 & $\cdots$ & $1.2 \pm 0.2$ & $1.5 \pm 0.4$ & $\cdots$ & $1.0 \pm 0.2$ & $\cdots$ & $2.4 \pm 0.6$ \\
\hline & 6 & 15 & $\cdots$ & $1.3 \pm 0.2$ & $1.5 \pm 0.4$ & $\cdots$ & $1.1 \pm 0.2$ & $\cdots$ & $3.0 \pm 0.4$ \\
\hline MACA & 0 & 6 & $\cdots$ & $1.3 \pm 0.2$ & $1.2 \pm 0.1$ & 1.8 & $0.5 \pm 0.1$ & $\cdots$ & $1.1 \pm 0.1$ \\
\hline & 6 & 6 & $\cdots$ & $1.0 \pm 0.1$ & $1.2 \pm 0.1$ & $\ldots$ & $0.5 \pm 0.1$ & $\ldots$ & $1.0 \pm 0.1$ \\
\hline GILA & 0 & 6 & $\cdots$ & $1.0 \pm 0.1$ & $1.9 \pm 0.2$ & $\ldots$ & $0.5 \pm 0.1$ & $\cdots$ & $1.6 \pm 0.2$ \\
\hline & 6 & 6 & $\cdots$ & $1.1 \pm 0.1$ & $1.7 \pm 0.2$ & 1.7 & $0.5 \pm 0.1$ & $\cdots$ & $1.8 \pm 0.1$ \\
\hline
\end{tabular}


San Manuel Smelter

\begin{tabular}{|c|c|c|c|c|c|c|c|c|c|}
\hline Site & $\begin{array}{r}\text { Depth } \\
\text { (in) }\end{array}$ & $\begin{array}{c}\# \\
\text { Samples }\end{array}$ & $\mathrm{Ti}$ & $\mathrm{U}$ & $v$ & $\mathbf{H}$ & Yb & $2 n$ & $2 r$ \\
\hline $\begin{array}{l}\sim 0.5 \mathrm{~km} \\
\sim 1 \mathrm{~km}\end{array}$ & $\begin{array}{r}0 \\
15 \\
0 \\
6\end{array}$ & $\begin{array}{l}4 \\
4 \\
2 \\
2\end{array}$ & $\begin{array}{c}5.2 \pm 1.6 \\
6.1 \pm 1.6 \\
2.8 \\
2.8\end{array}$ & $\begin{array}{c}1.7 \pm 0.1 \\
2.5 \pm 0.4 \\
1.3 \\
1.4\end{array}$ & $\begin{array}{c}4.6 \pm 1.1 \\
5.8 \pm 1.4 \\
2.0 \\
2.2\end{array}$ & $\begin{array}{l}\cdots \\
\cdots \\
\cdots \\
\cdots\end{array}$ & $\begin{array}{c}3.2 \pm 0.6 \\
4.0 \pm 0.8 \\
2.0 \\
1.8\end{array}$ & $\begin{array}{c}6.3 \pm 2.7 \\
4.1 \pm 1.1 \\
5.2 \\
2.6\end{array}$ & $\begin{array}{l}\ldots \\
\ldots \\
\ldots\end{array}$ \\
\hline $\begin{array}{l}\sim 2 \mathrm{~km} \\
-5 \mathrm{~km}\end{array}$ & $\begin{array}{r}0 \\
15 \\
0 \\
15\end{array}$ & $\begin{array}{l}3 \\
3 \\
3 \\
3\end{array}$ & $\begin{array}{l}3.7 \pm 0.6 \\
3.7 \pm 0.3 \\
3.4 \pm 1.0 \\
4.0 \pm 0.5\end{array}$ & $\begin{array}{l}1.7 \pm 0.1 \\
1.5 \pm 0.2 \\
1.3 \pm 0.3 \\
1.3 \pm 0.2\end{array}$ & $\begin{array}{l}3.3 \pm 0.2 \\
2.8 \pm 0.2 \\
2.2 \pm 1.0 \\
2.7 \pm 0.3\end{array}$ & $\begin{array}{l}\cdots \\
\cdots \\
\cdots\end{array}$ & $\begin{array}{l}3.0 \pm 0.2 \\
2.9 \pm 0.6 \\
1.8 \pm 0.6 \\
2.0 \pm 0.1\end{array}$ & $\begin{array}{l}4.1 \pm 0.3 \\
2.4 \pm 0.2 \\
2.2 \pm 0.6 \\
2.4 \pm 0.4\end{array}$ & $\begin{array}{l}\cdots \\
\ldots \\
\ldots\end{array}$ \\
\hline
\end{tabular}

SEquaro National Monument

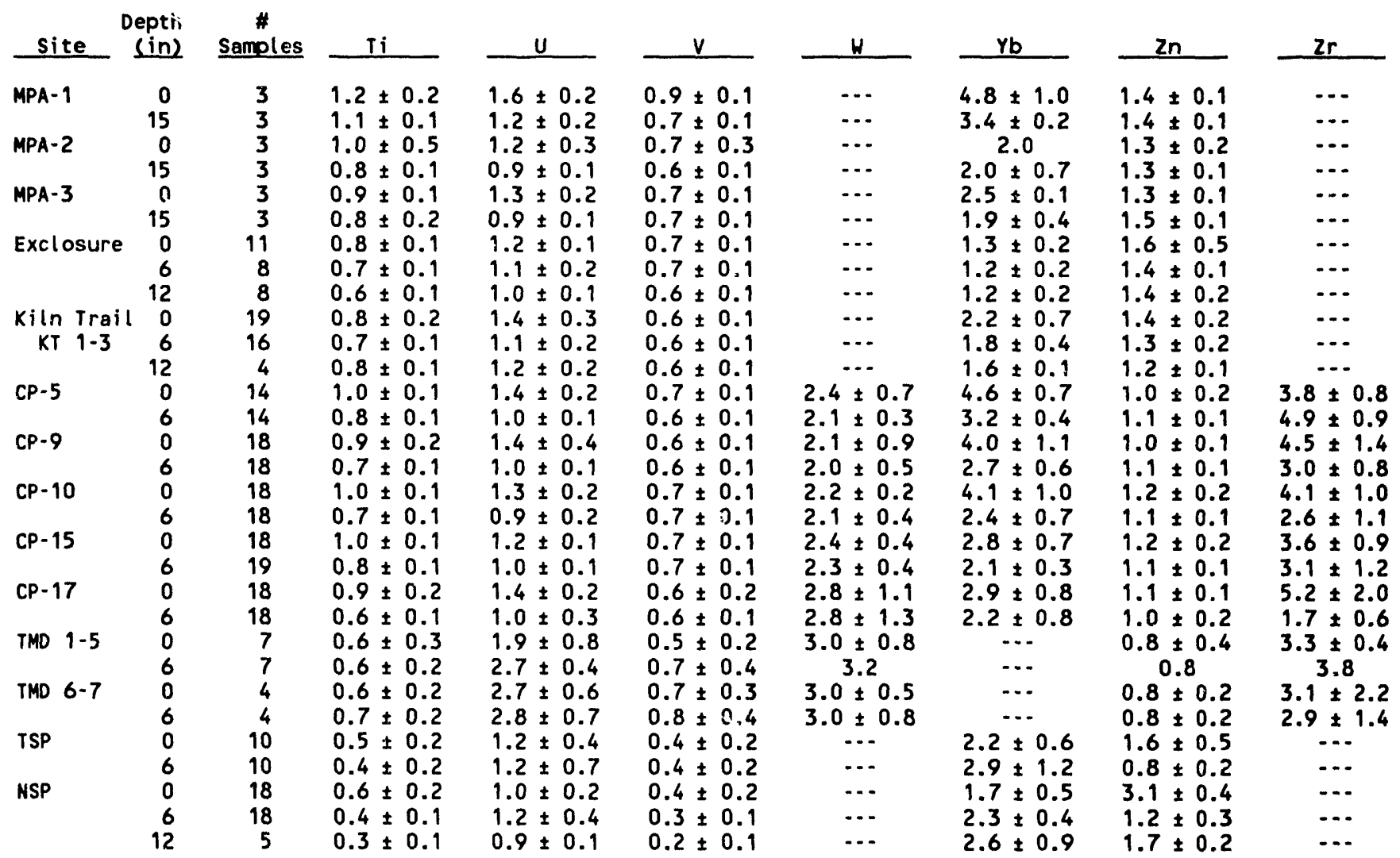

Regional Background

\begin{tabular}{|c|c|c|c|c|}
\hline site & $\begin{array}{r}\text { Depth } \\
\text { (in) }\end{array}$ & $\begin{array}{c}\# \\
\text { Samples }\end{array}$ & $\mathrm{Ii}$ & $\underline{U}$ \\
\hline CATAL & $\begin{array}{r}0 \\
6 \\
12\end{array}$ & $\begin{array}{l}3 \\
3 \\
3\end{array}$ & $\begin{array}{c}0.4 \pm 0.2 \\
0.4 \\
0.2\end{array}$ & $\begin{array}{l}0.7 \pm 0.2 \\
0.6 \pm 0.1 \\
0.6 \pm 0.1\end{array}$ \\
\hline CHIR & $\begin{array}{l}0 \\
6\end{array}$ & $\begin{array}{l}15 \\
15\end{array}$ & $\begin{array}{l}0.7 \pm 0.2 \\
0.6 \pm 0.2\end{array}$ & $\begin{array}{l}2.0 \pm 0.4 \\
2.3 \pm 0.3\end{array}$ \\
\hline HACA & $\begin{array}{l}0 \\
6\end{array}$ & $\begin{array}{l}6 \\
6\end{array}$ & $\begin{array}{l}1.5 \pm 0.2 \\
1.2 \pm 0.1\end{array}$ & $\begin{array}{l}0.9 \pm 0.1 \\
0.8 \pm 0.1\end{array}$ \\
\hline GILA & $\begin{array}{l}0 \\
6\end{array}$ & $\begin{array}{l}6 \\
6\end{array}$ & $\begin{array}{l}0.9 \pm 0.2 \\
1.0 \pm 0.1\end{array}$ & $\begin{array}{l}1.1 \pm 0.2 \\
1.2 \pm 0.1\end{array}$ \\
\hline
\end{tabular}

\begin{tabular}{cc}
$V$ & $U$ \\
\cline { 2 - 2 } $0.3 \pm 0.2$ & $\ldots$ \\
$0.1 \pm 0.1$ & $\cdots$ \\
0.1 & $\cdots$ \\
$0.4 \pm 0.1$ & $\cdots$ \\
$0.3 \pm 0.1$ & $\cdots$ \\
$1.3 \pm 0.1$ & $\cdots$ \\
$1.3 \pm 0.1$ & $\cdots$ \\
$0.6 \pm 0.1$ & $\cdots$ \\
$0.7 \pm 0.1$ & $\cdots$
\end{tabular}

$-12-$

\begin{tabular}{l}
$Y b$ \\
\hline $0.8 \pm 0.2$ \\
$0.6 \pm 0.2$ \\
$0.9 \pm 0.2$ \\
$1.5 \pm 0.3$ \\
$1.7 \pm 0.3$ \\
$1.1 \pm 0.2$ \\
$0.9 \pm 0.1$ \\
$1.8 \pm 0.3$ \\
$1.8 \pm 0.4$
\end{tabular}
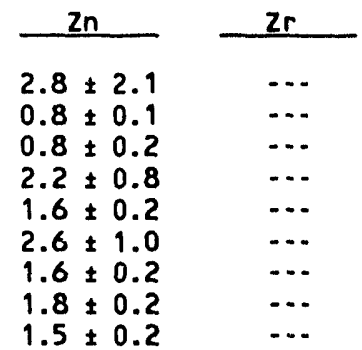
"salt" exposure, because these readily mobile elements seem to have been effectively leached from the soils studied. The third group (within normal natural ranges) has the largest membership: $\mathrm{Ba}$, $\mathrm{Br}, \mathrm{Ce}, \mathrm{Cr}, \mathrm{Dy}, \mathrm{Eu}, \mathrm{Fe}, \mathrm{F}, \mathrm{Ga}, \mathrm{K}, \mathrm{La}, \mathrm{Lu}, \mathrm{Mn}, \mathrm{Na}, \mathrm{Nd}, \mathrm{Rb}, \mathrm{Sc}, \mathrm{Si}, \mathrm{Sm}, \mathrm{Sr}, \mathrm{Ta}, \mathrm{Tb}, \mathrm{Ti}, \mathrm{U}, \mathrm{V}, \mathrm{Yb}$, and $\mathrm{Zn}$. We have discovered little, if any, evidence that their concentrations in SAGU soils are being affected by any anthropogenic source.

The fourth group, those with EFs consistently in excess of 2.5, contain $s$ me unusual members. We place As, $\mathrm{Cs}, \mathrm{Hf}, \mathrm{In}, \mathrm{Sb}, \mathrm{Se}, \mathrm{Th}, \mathrm{W}$, and $\mathrm{Zr}$ in this group; they require further scrutiny concerning possible non-natural sources for their elevated levels (relative to Wedepohl's crustal average) observed in many or most samples taken during this study. Concentrations of these elements (except Cs, In, and Se) on the Tucson Mo intain District samples that were separated into four particle-size groups also exhibited mark ad preference for the smallest size fraction of soil particles. This latter phenomenon suggests that the enrichment of six of these elements may be related, although $\mathrm{Hf}, \mathrm{Th}, \mathrm{W}$, and $\mathrm{Zr}$ are rarely found in excess concentrations in air-borne emissions (except for some municipal incinerator air particulates in the Eastern US, Ref. 21). These four elements are associated and enriched in naturally-occurring zircons and zeolites. These minerals are highly resistant to chemical weathering and may be ground to fine particles that are retained for extended time periods in this ecosystem. This is in concert with their system-wide higher EFs. We have no explanation for the unusually high EFs observed for Cs in the San Manuel Canyon and SAGU Cactus Forest. Ordinarily, this element has no air pollution input or implications.

Indium, $\mathrm{Sb}$, and $\mathrm{Zn}$ are significantly enriched in the smelter canyon, in the SAGU soils from the RMD unit, and in many of the regional "background" sites. These elements are more strongly enriched in the surface soils of the smelter canyon, and are commonly associated with the roasting process for sulfide ore oxidation and metal recovery. There is evidence for regional impact for these elements, probably from the metal smelting industry in southern Arizona. Throughout the regional samples we examined, these elements are significantly more enriched in the surface soils relative to the subsurface soils, implying that air pollutant deposition can be a major source. Arsenic, another element commonly associated with copper ore reduction, does not yield such a clear-cut interpretation. The problem is largely due to arsenic concentrations being near our detection limits, thereby reducing our ability to make a meaningful regional assessment of its distribution. Arsenic is a well-known air pollutant arising from the metal smelting industry, and we suspect that it would show a similar distribution to $\mathrm{Sb}$, were our analytical method more sensitive for this important element. Furthermore, before the widespread use of neutron activation analysis, Sb was often measured with relatively poor precision and accuracy in geological materials. The crustal abundance of this element merits recalculation.

\section{SUMMARY AND MANAGEMENT IMPLICATIONS}

Previous application of the EF approach to the air particulate data taken on the dichotomous sampler at the SAGU air monitoring station clearly indicates that certain elements $(\mathrm{Mg}, \mathrm{Na}, \mathrm{Ca}$, $\mathrm{Zn}, \mathrm{Br}, \mathrm{Pb}, \mathrm{S}$, and $\mathrm{Cu}$ ) are currently enriched on the fine fraction of air particulates coming into 
the Monument (19). Similarly, several trace elements in the soil samples taken as part of this study also indicate anthropogenic impact, probably from dry deposition of air pollutants. The present soil data do not reflect the apparent enrichment of $\mathrm{Mg}, \mathrm{Na}, \mathrm{Ca}$, and $\mathrm{Br}$ observed on the fine particle fraction. Zinc was shown to be enriched in some of our SAGU soil samples, 'while the other enriched elements from the air particulate study were not determined in our samples.

Clearly, the saguaro cactus resource (and perhaps other cactus species) are undergoing a continuing decline within the SAGU ecosystem. While our data do not implicate anthropogenic air pollution impact for inorganic elements, atmospheric emissions related to the Tucson metropolitan area cannot yet be disregarded as a potential cause for the decline. The complex nature of anthropogenic modification of the organic compound burden of atmospheric aerosols is just beginning to be understood. Direct impact from the leeward drift of agricultural chemicals (herbicides and pesticides) from air-borne spraying operations or other organic chemical enrichment from diffuse urban sources should be considered a high priority for future investigation as a possible mechanism to explain the observed decline in the cactus populations. Unfortunately, organic chemic: 1 analyses in natural materials are far more difficult and expensive to conduct than inorganic determinations, and the spectrum of compounds runs into the millions. There has also been some preliminary evidence from unpublished work conducted by colleagues at LANL that short-chained organic acids readily penetrate the waxy cuticles of cacti, perhaps having a here-to-fore unanticipated "acid-rain" effect on desert ecosystems (22). Evans (23) has also speculated on the permeability of waxy cuticles by polar vs. non-polar compounds.

\section{ACKNOWLEDGMENTS}

The success of our Interagency Agreement has been a result of ongoing efforts by our project managers, Ken Stolte, Kathy Tonnessen, and Tanni Manerio of the NPS Air Quality Division. We also want to recosisize the enthusiastic cooperation from NPS staff in Saguaro National Monument: Bill Palik, Superintendent, emeritus; Robert Hall, Chief of Resource Management, emeritus; Bobbi Simpson and Shelly Sparhawk from the Resource Management Staff; and Jim Young, a Park Volunteer in 1990. In addition, we appreciate the generous assistance from Don Graybill, Frank Telewski, and all the staff at the Tree Ring Research Laboratory of the University of Arizona. We thank the NPS staffs at the background sites: Chiricahua, Gila Cliffs, and Walnut Canyon National Monuments. We also thank Christy Gladney for her assistance with some of the sample collection and preparation.

\section{REFERENCES}

(1) A. B. Clemensen, "Cattle, Copper, and Cactus: The History of Saguaro National Monument," National Park Service Report, U. S. Government Printing Office, Denver, 271 pp. (1987).

(2) R. M. Turner, S. M. Alcorn, G. Olin, and J. A. Booth, "The Influence of Shade, Soil, and Water on Saguaro Seedling Reestablishment," Botanical Gazette, 127, 95-102 (1966). 
(3) R. M. Turner, "Long-term Vegetation Change at a Fully Protected Sonoran Desert Site," Ecology, 71, 464-477 (1990).

(4) D. A. Graybill and M. R. Rose, "Analysis of Growth Trends and Variation in Conifers from Central Arizona: I. Network Chronology Development and Analysis," Laboratory of Tree Ring Research, final report, University of Arizona, Tucson, Arizona (1989).

(5) K. W. Stolte, "Air Pollution Threats to Biological Resources in Saguaro National Monument," in Proceedings of the Symposium on Research in Saguaro National Momument, C. Stone and E. Bellantoni, Editors, (Southwest Parks and Monuments Association, Tucson, Arizona, 1992).

(6) E. S. Gladney, D. B. Curtis, D. R. Perrin, J. W. Owens, and W. E. Goode, "Nuclear Techniques for the Chemical Analysis of Environmental Materials," Los Alamos Scientific Laboratory report LA-8192-MS (1980).

(7) S. R. Garcia, W. K. Hensley, M. M. Minor, M. M. Denton, and M. A. Fuka, "An Automated Multidetector System for Instrumental Neutron Activation Analysis of Geological and Environmental Materials," in Atomic and Nuclear Methods in liossil linergy Research, R. H. Filby, B. S. Carpenter, and R. C. Ragaini, Editors, pp. 133-140 (1982).

(8) M. A. Gautier and E. S. Gladney (editors), "Health and Environmental Chemistry: Analytical Techniques, Data Management, and Quality Assurance," Los Alamos National Laboratory report LA-10300-M, Vols. I and II (1986).

(9) M. A. Gautier, E. S. Gladney, M. B. Phillps, and B. T. O'Malley, "Quality Assurance for Health and Environmental Chemistry: 1988," Los Alamos National Laboratory report LA-11637-MS, 479 pp. (1989)

(10) A. Stern, Air Pollution (Academic Press, New York, 1968)

(11) G. Hidy, Aerosols: An Industrial and Limironmental Science (Academic Press, NY, 1984)

(12) J. Seinfeld, Atmospheric ('hemistry and Physics of Air Pollution (Wiley, New York, 1987)

(13) E. S. Gladney, "Trace Element Emissions from a Coal-fired Power Plant: A Study of the Chalk Point Electric Generating Station," Ph.D. thesis, Dept of Chemistry, University of Maryland, 350 pp. (1974)

(14) G. E. Gordon, W. H. Zoller, E. S. Gladney, and R. R. Greenberg, "The Use of Instrumental Nuclear Activation Methods in the Study of Particulates from Major Air Pollution Sources," in Proceedings of the Second International ('onference on Nuclear Methods in linvirommental Research, J. R. Vogt and W. Meyer, Editors (University of Missouri, Columbia, Missouri, 1974) pp. $344-353$ 
(15) M. Gallorini, E. Orvini, A. Rolla, and M. Burdisso, "Destructive Neutron Activation Analysis of Toxic Elements in Suspended Materials Released from Refuse Incinerators," Analyst, 106, 328-334 (1981).

(16) J. M. Ondov, W. H. Zoller, and G. E. Gordon, "Trace Element Emissions on Arrosols from Motor Vehicles," Environmental Science and Technology, 16, 318-328 (1982)

(17) G. E. Gordon, W. H. Zoller, E. S. Gladney, and A. G. Jones, "Trace Elements in the Urban Atmosphere," in P'roceedings of the American Nuclear Society Topical Meeting on Nuclear Methods in linvironmental Research, J. Vogt, Editor (University of Missouri, Columbia, Missouri, 1971), pp. 30-37.

(18) G. E. Gordon, W. H. Zoller, and E. S. Gladney, "Abnormally Enriched Trace Elements in the Atmosphere," in Trace Substances in linvironmental Health, VII, D. D. Hemphill, Editor (University of Missouri, Columbia, Missouri, 1973), pp. 167-174.

(19) E. S Gladney, "Origins and Effects of Dry-deposited Minerals in Desert Ecosystems: Some Atmospheric Chemistry Considerations," in Acid Rain and Air Pollution in Desert Park Areas: Proceedings of a Workshop, 16-18 May 1988, and Management Recommendations, Tucson, Arizona, Technical Report NPS/NRAQD/NP.TR-91/02, pp. 52-67 (1991).

(20) K. H. Wedepohl, (rigin and Distribution of the Lilements, L. H. Ahrens, Editor (Pergamon Press, London, 1968), pp. 999 - 1016.

(21) R. R. Greenberg, "Trace Element Emissions from Municipal Incinerators," Ph D. thesis, Dept. of Chemistry, University of Maryland (1976)

(22) E. Gaffney, Private C`mmunication, Los Alamos National Laboratory (1988).

(23) L. . Evans, "Acidic Precipitation Effects on Terrestrial Vegetation," Ann. Rev. Phytopathol., 22, 397-420 (1984).

(24) E. S. Gladney, B. T. O'Malley, I. Roelandts, and T. E. Gills, "Compilation of Elemental Concentration Data for NBS Clinical, Biological, Geological and Environmental Standari Reference Materials," NBS Special Publication 260-111 (1987).

(25) E. S. Gladney and I. Roelandts, "1988 Compilation of Elemental Concentration Data for CCRMP Soils SO-1 to SO-4," Geostandards Newsletter, 13, 21 7-268 (1989).

(26) E. S. Gladney and I. Roelandts, "1988 Compilation of Elemental Concentration Data for CCRMP Reference Rock Samples SY-2, SY-3, and MRG-1," Geostandards Newsletter, 14, 373 458 (1990) 
Appendix A: Sampling Site Locations

$-17-$ 


\section{Sampling Site Locations}

Sites selected for this study included three regional background sites, one local background site, seven sites in the western portion of the Monument (Tucson Mountain District), four areas associated with the Tree Ring Lab's growth suppression study, five cactus diversity monitoring plots, a variety of sites within the Cactus Forest section of the eastern portion of the Monument (Rincon Mountain District), and the San Manuel Smelter Canyon northeast of the Catalina Mountains.

Acronym

CHIR Rhyolite Canyon, Chiricahua National Monument, Arizona

Gil.A Gila Cliffs National Monument, New Mexico

WACA Walnut Canyon National Monument, Arizona

CATA Catalina Mountains, Arizona

SMEL.TER San Manuel Smelter Canyon

RMI)

('SP

(P-5

(P.9

(P-10

CP-15

CP.17

EXCl.

KT

HDP

NSP

MPA

HVP

RPP

TSP

TMD
Rincon Mountain District, Saguaro National Monument (East)

Cowhead Saddle, Tanque Verde Ridge, RMD

RMD Cactus Plot \# 5 (1989-1990)

RMD Cactus Plot \# 9 (1989-1990)

RMD Cactus Plot \#10 (1989-1990)

RMD Cactus Plot \#15(1989-1990)

RMD Cactus Plot \# 17 (1989-1990)

Exclosure Site, RMD

Kiln Train, Cactus Forest, RMD

Helen's Dome, Ponderosa and White Pine, RMD

North Slope, Ponderosa Pine, White Fine,

and Douglas Fir, RMD

Mica Picnic Area, RMD

Happy Valley Saddle, RMD

Rincon Peak, RMD

Tucson Side, Ponderosa and White Pine, RMD

Tucson Mountain District, Saguaro National Monument (West)
Figures

2, A.1, A-2

2, A..3, A-4

2, A-5

2

A-6

1. A-7

A-7

A-8

A-8

A.8

A 9

A.8

A.8

A-8

A-7, A-9 $\wedge-10$

A-7, A-11

A- 12

A-8

A.7

A-7

A-7, A-13

1. A-14 


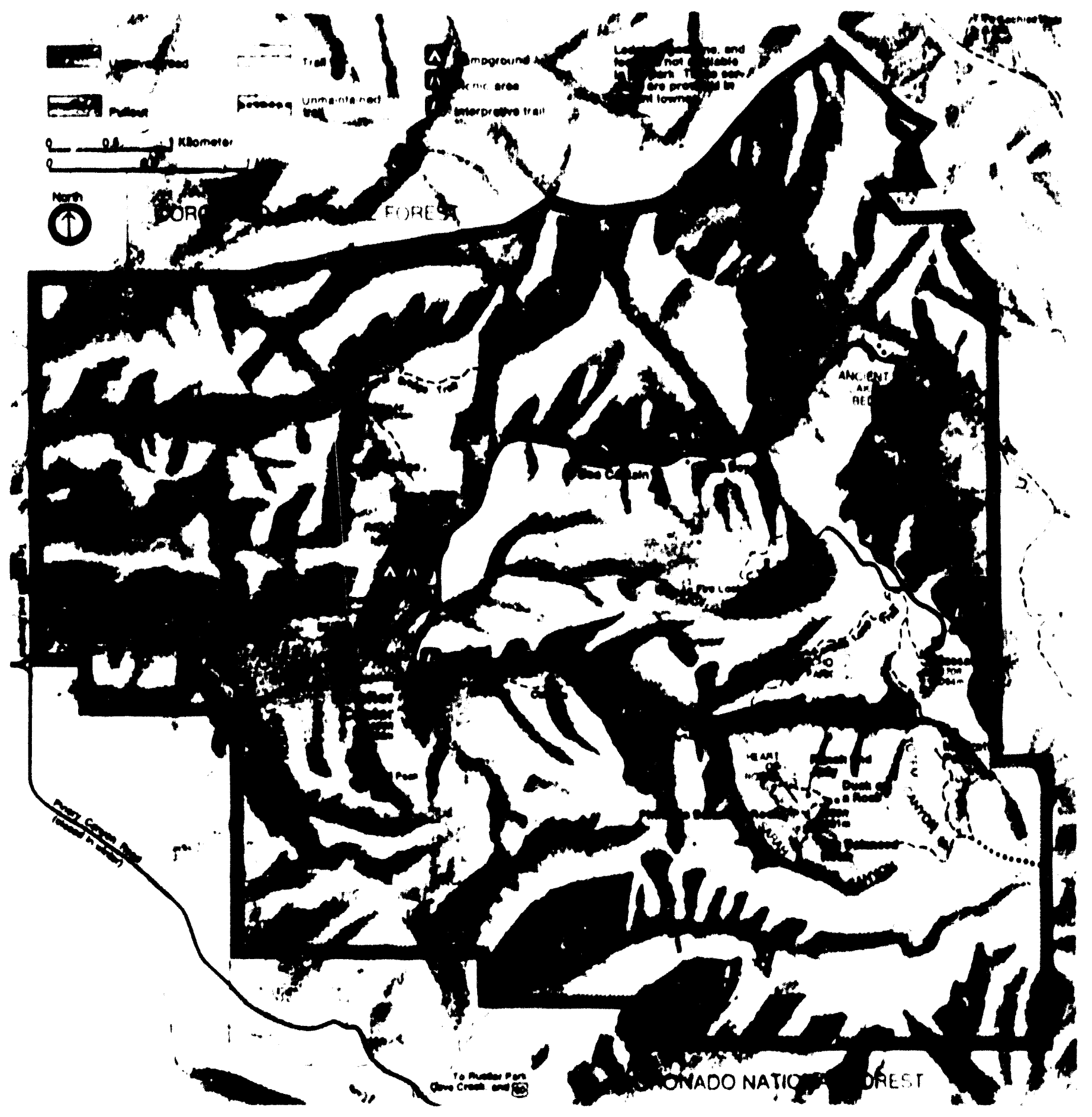

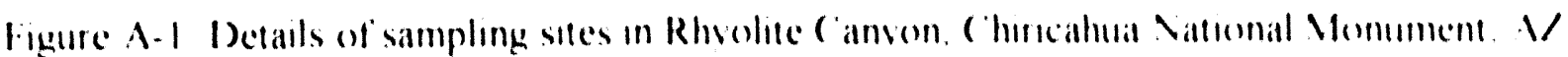




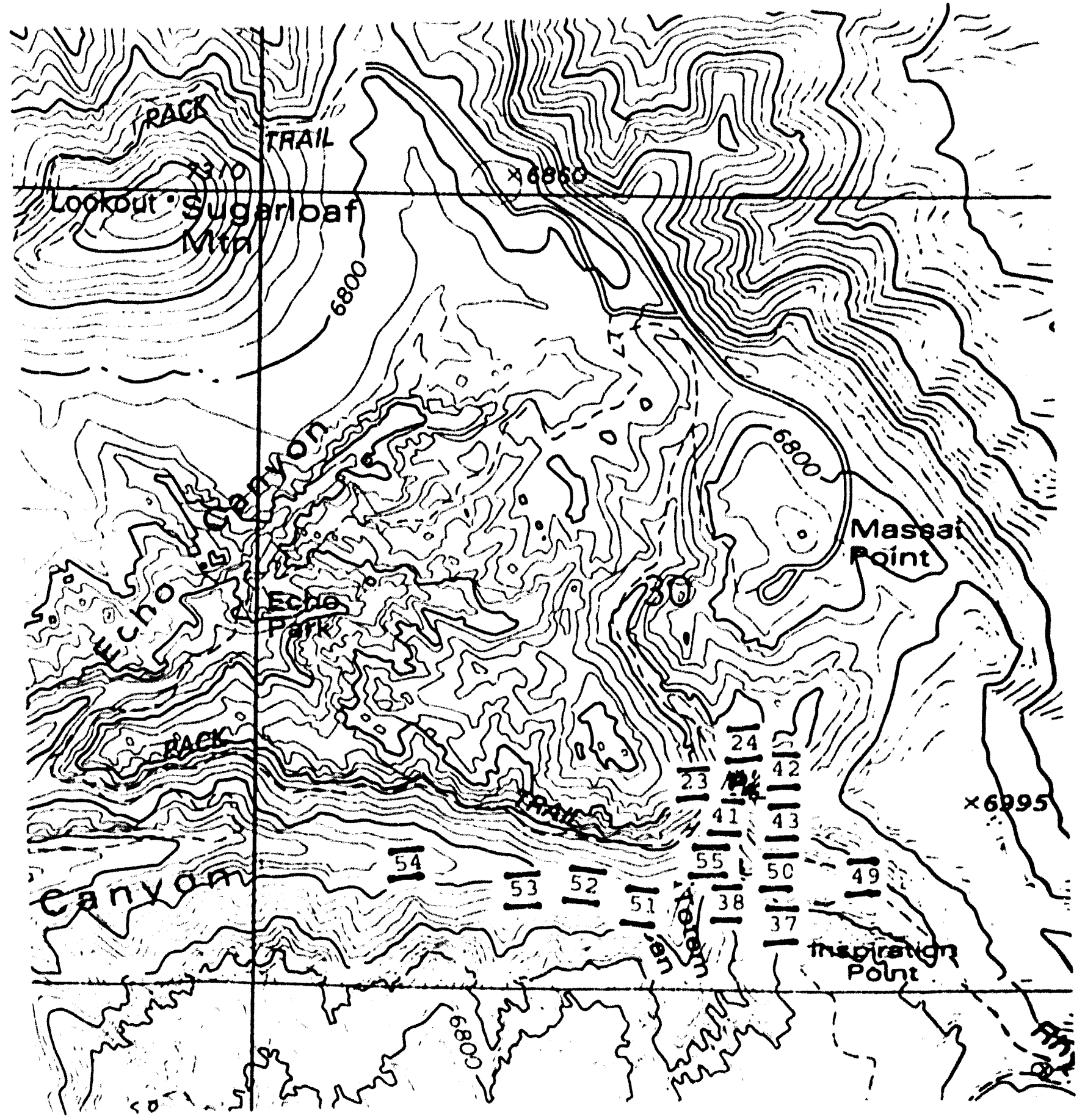

Figure A-2 Details of sampling sites in Rhyolite ('anyon, (hiricahua National Monument, $\mathrm{AZ}$. 


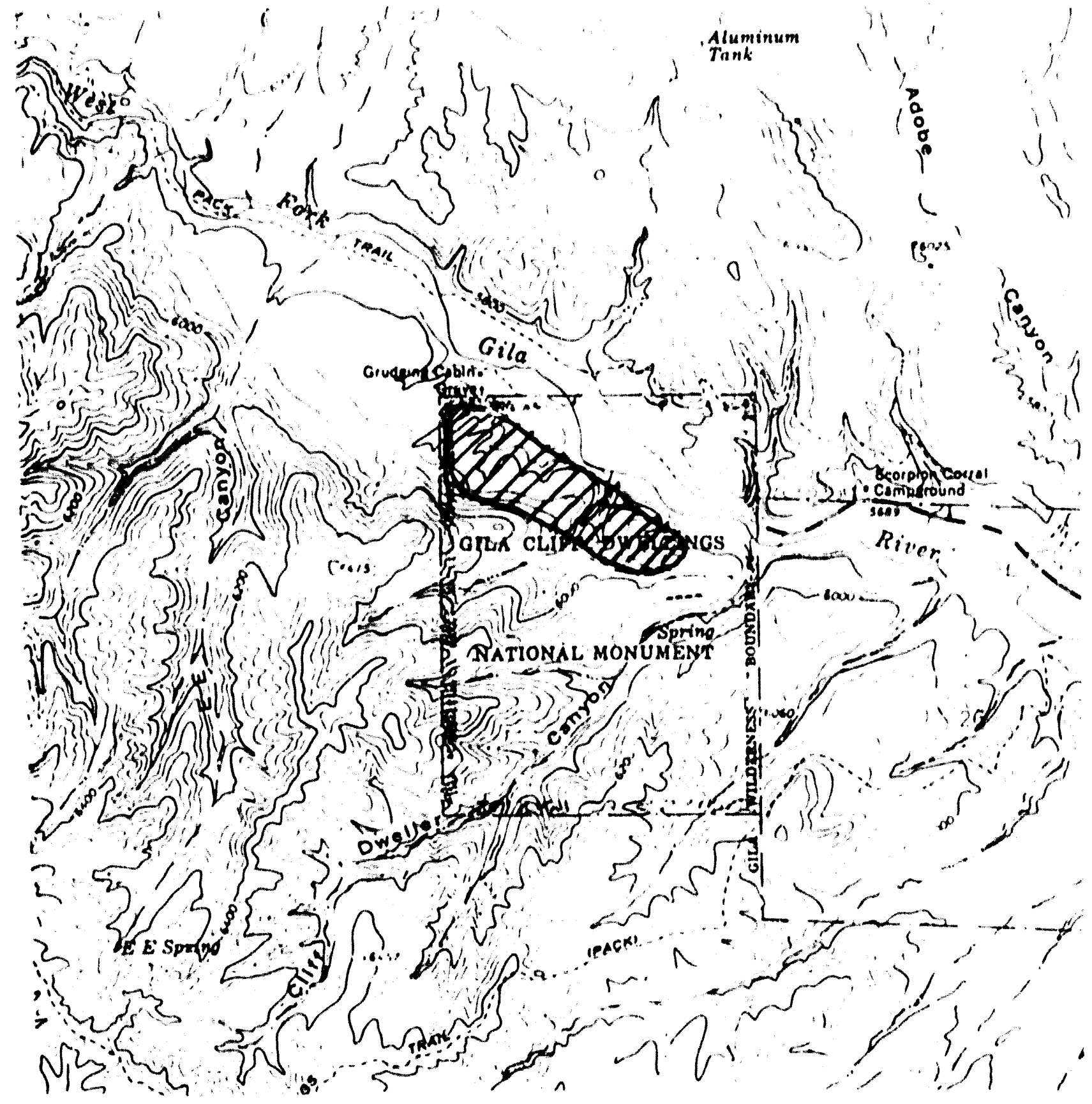

Figure A-3 Details of sampling sites in (jila Cliffs National Monument, New Mexico 


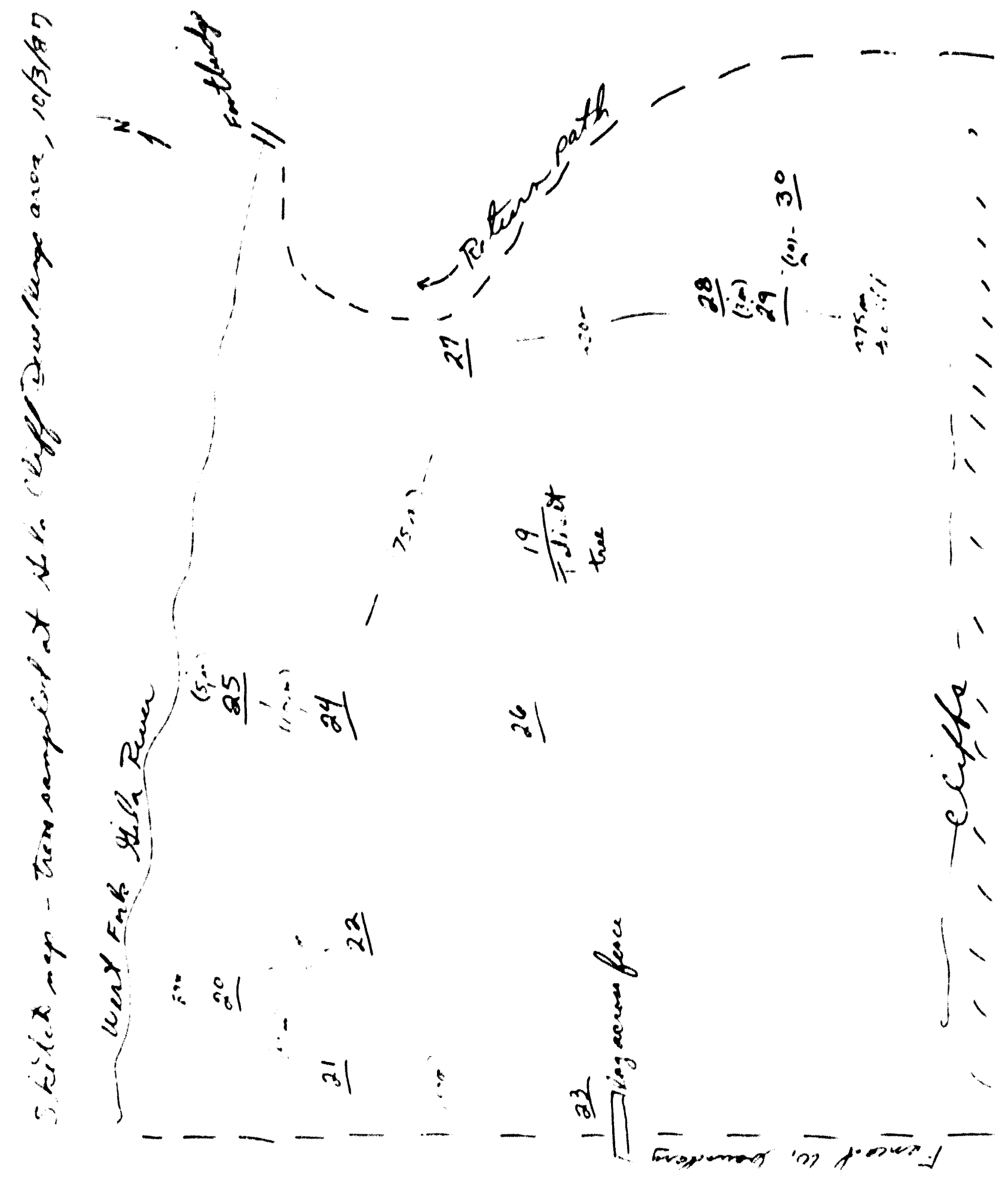

Figure A-4. D tails of sampling sites in Gila Cliffs National Monument, New Mexico 


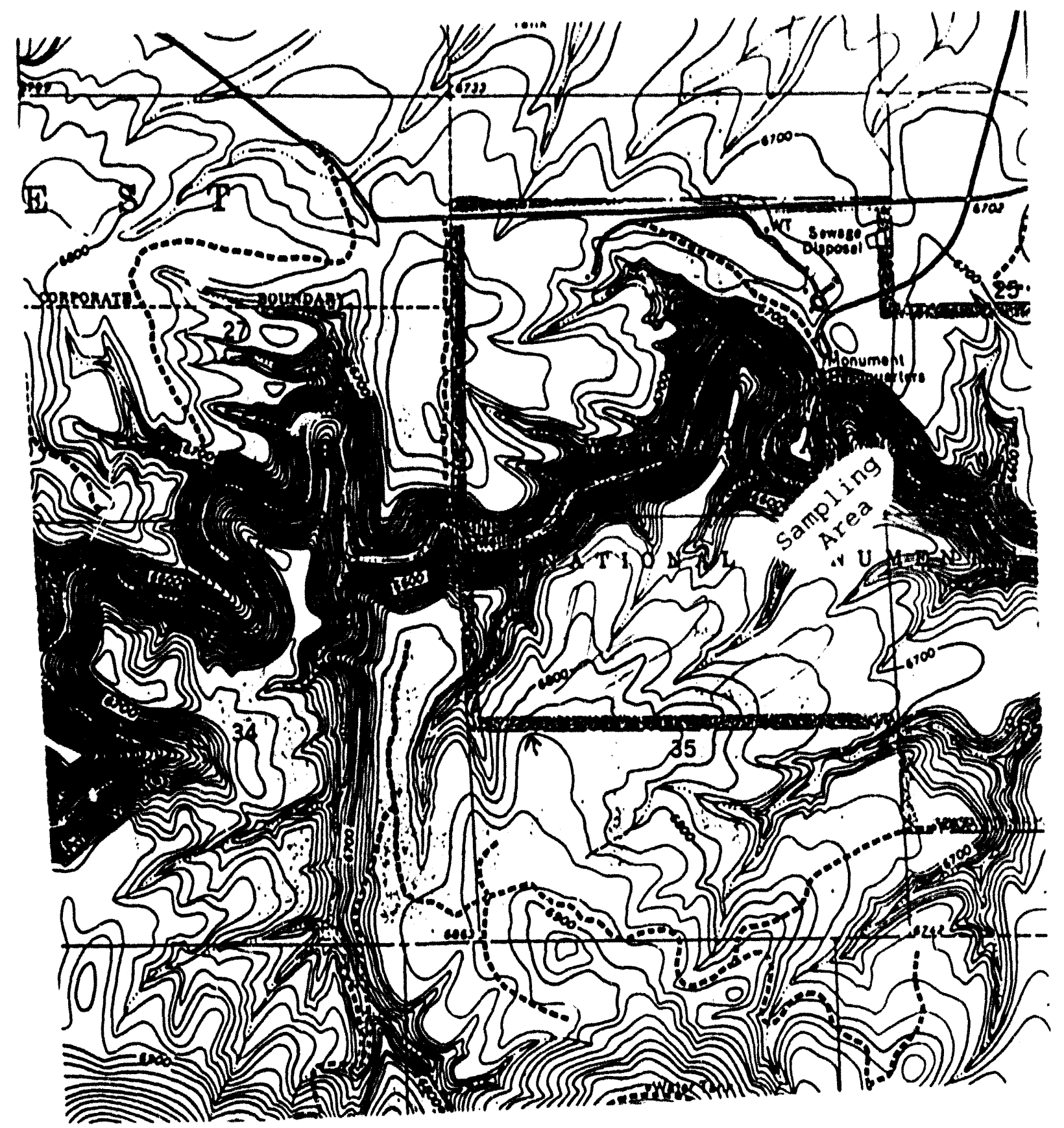

Figure A-5 Details of sampling sites in Walnut Canyon National Monument. Arizona 


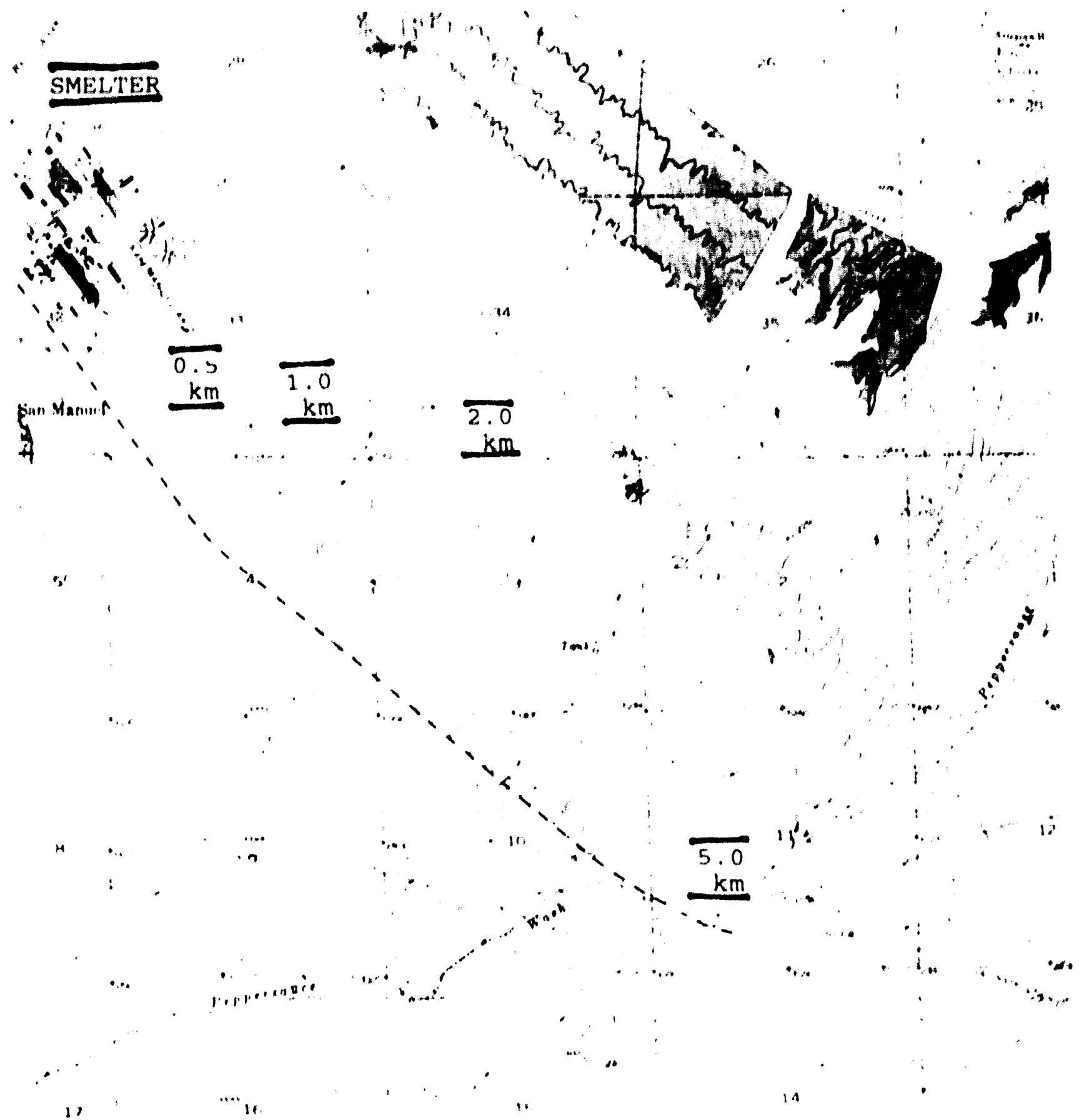

Figure A-6 Details of sampling sites in San Manuel Smelter ('anyon, Arizona 


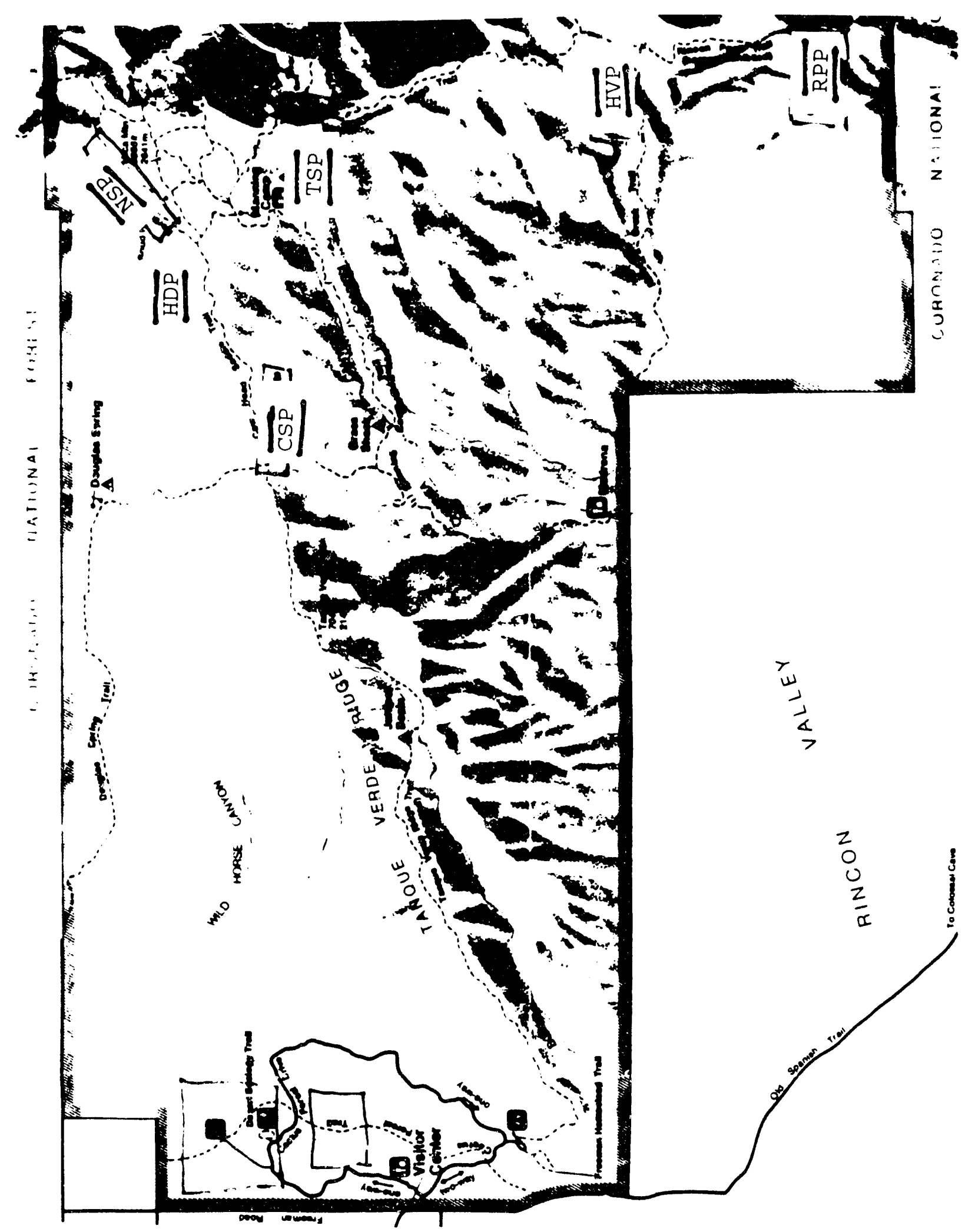

Figure A-7. Overview of sampling sites in RMD, Saguaro National Monument (East), AZ. 


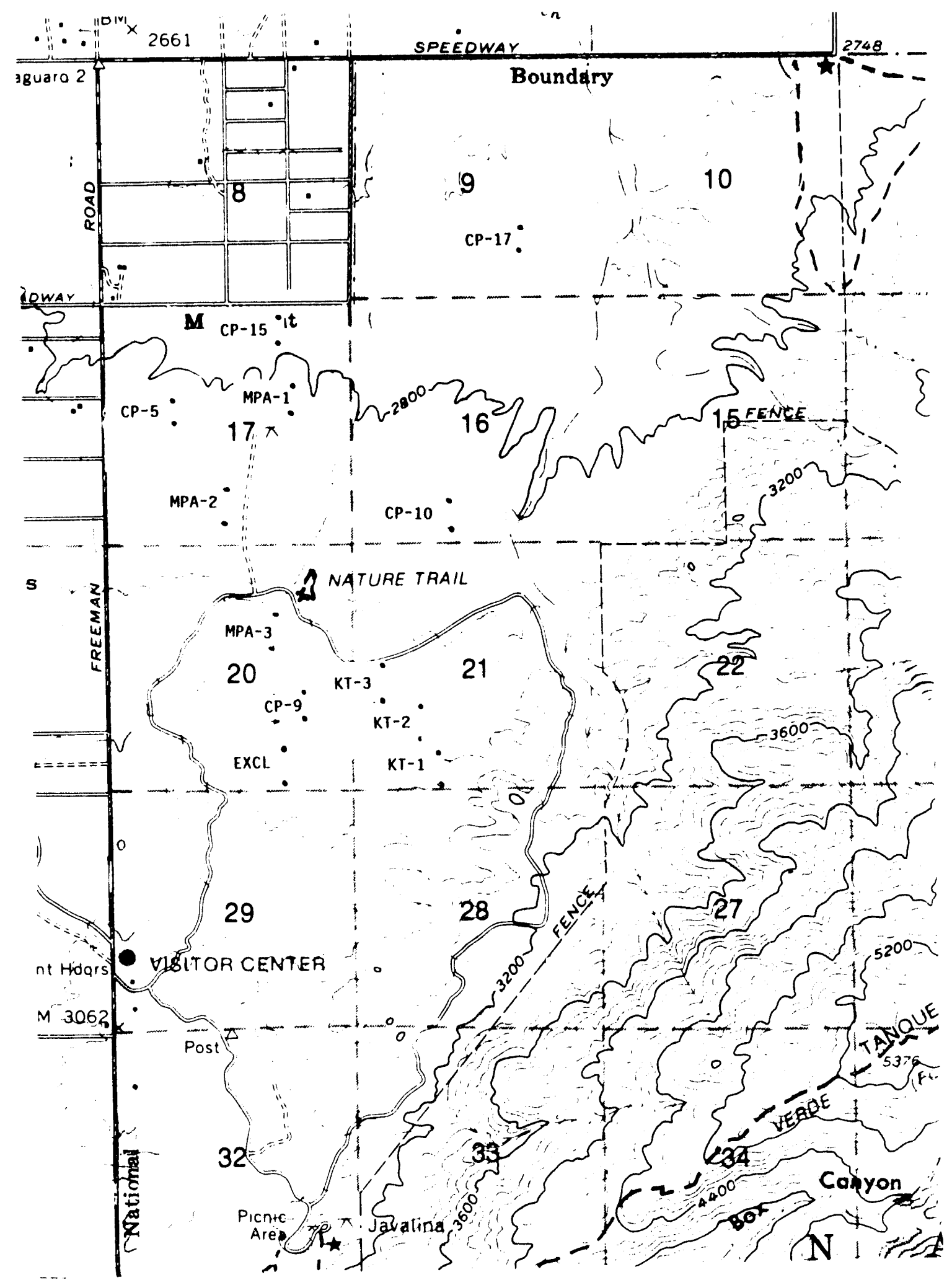

Figure A-8. Details of sampling sites in Cactus Forest, RMD. 


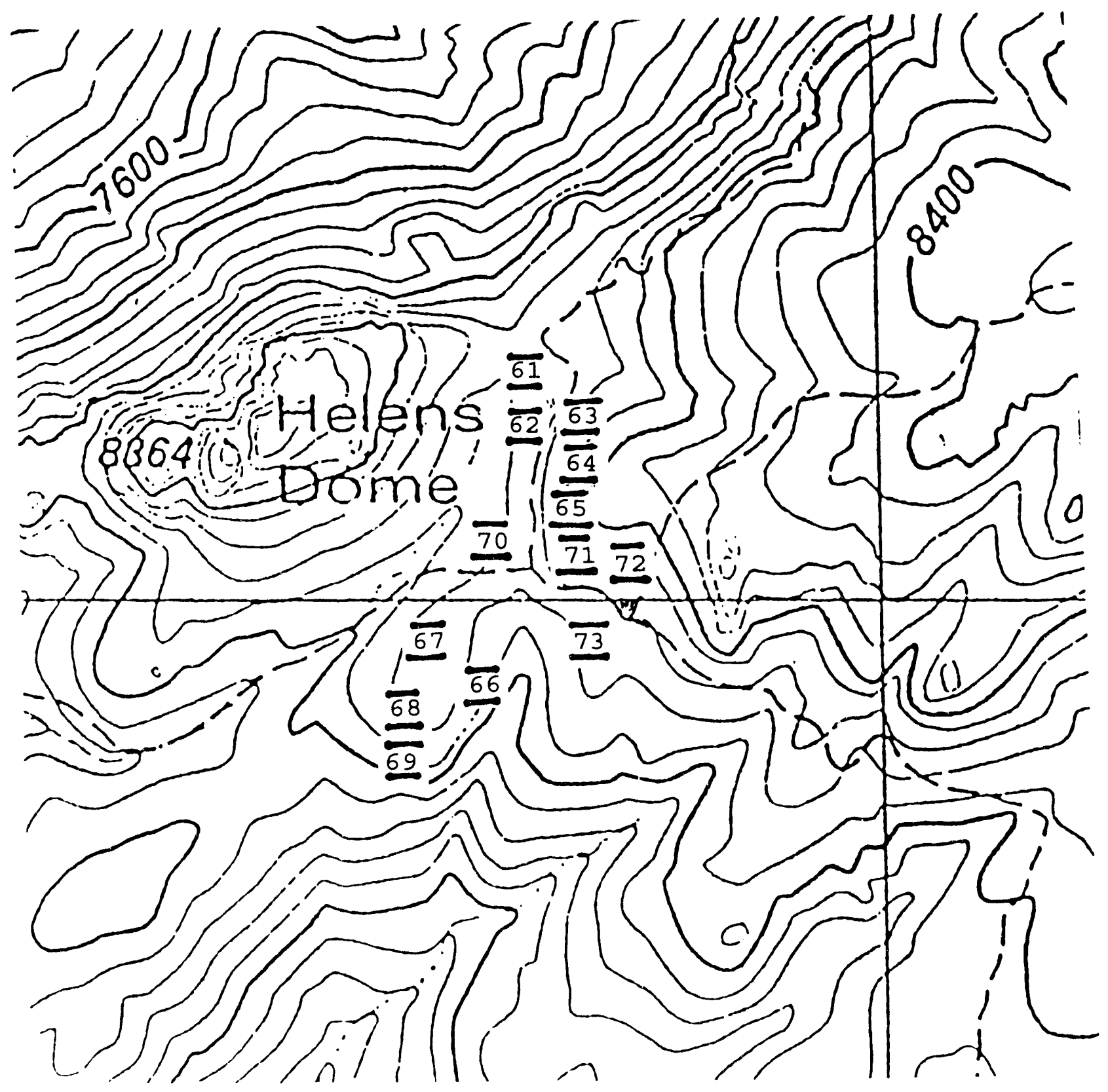

Figure A-9. Details of sampling sites in Helen's Dome, Ponderosa and White Pine, RMD. 


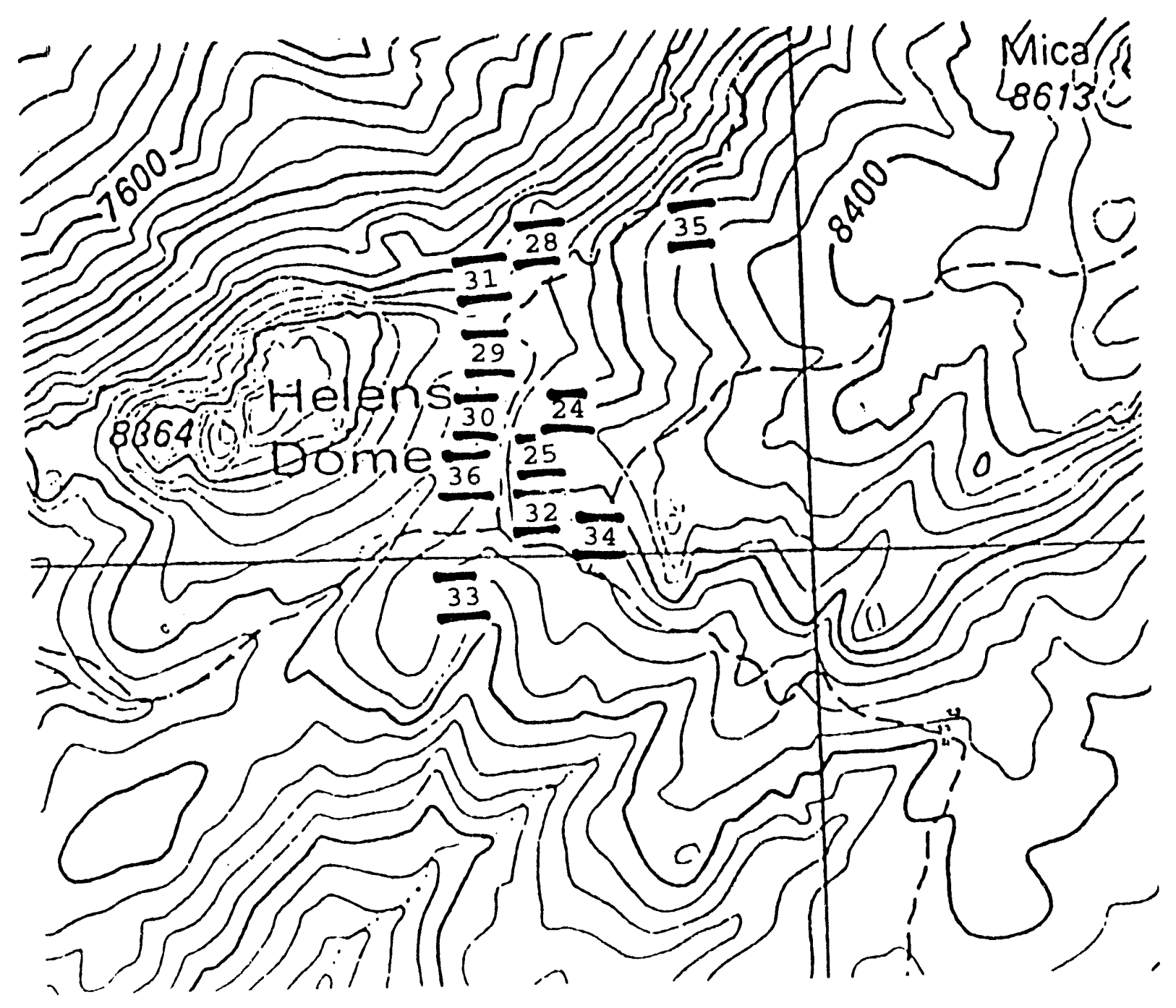

Figure A-10. Details of sampling sites in Helen's Dome, Ponderosa and White Pine, RMD. 


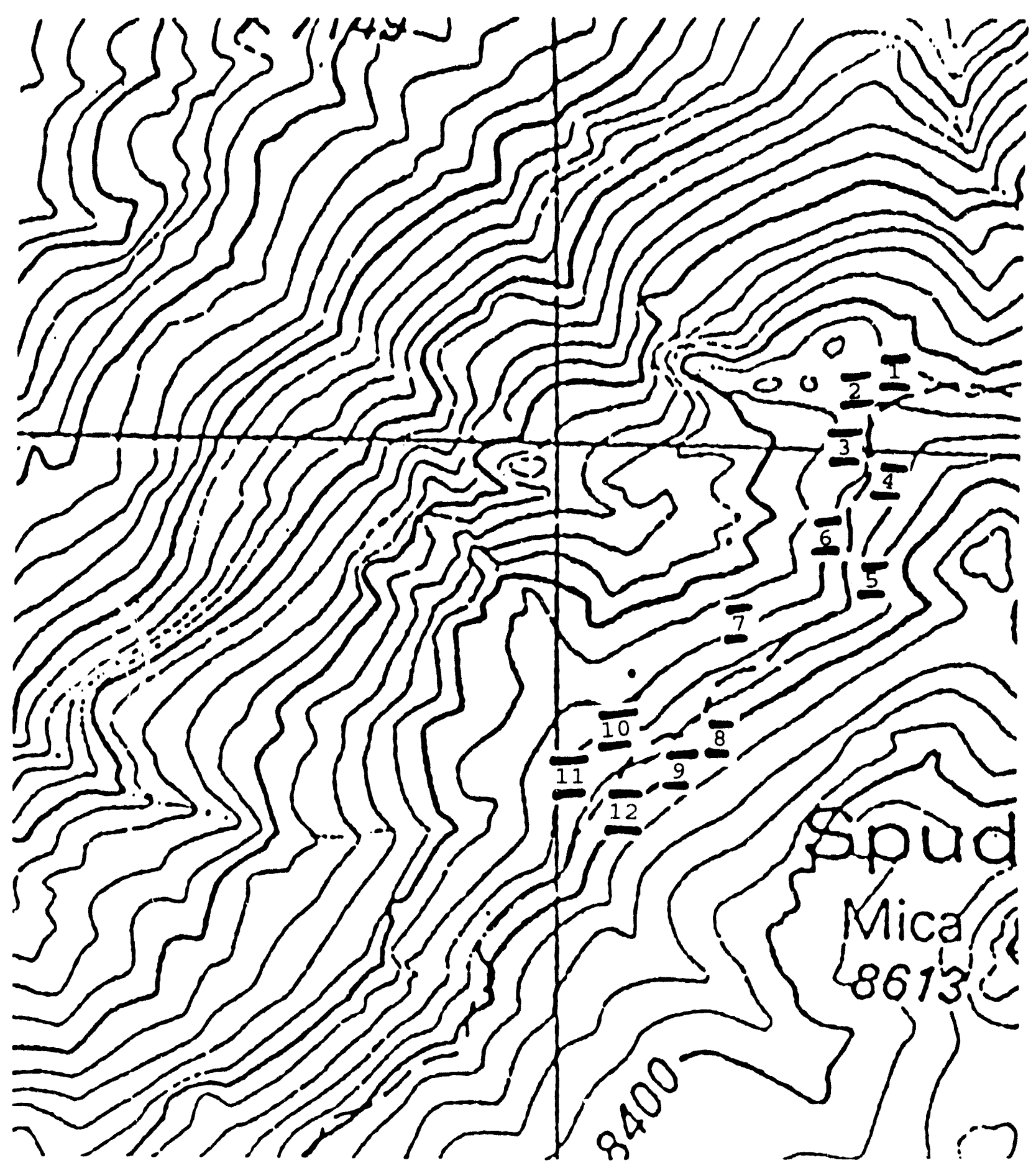

Figure A-11. Sampling sites in North Slope, Ponderosa Pine, White Pine, and Douglas Fir, RMD 


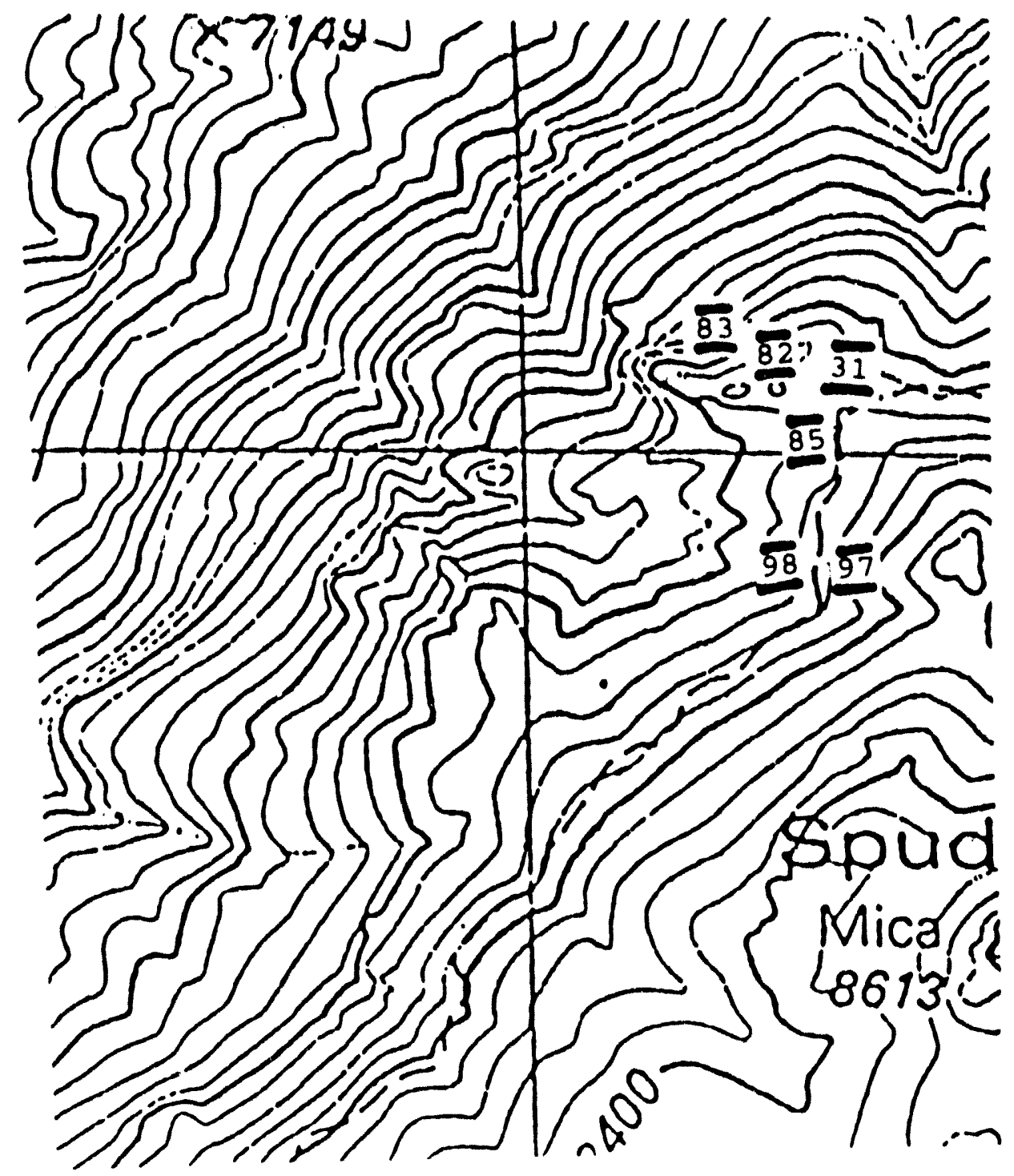

Figure A-12. Sampling sites in North Slope, Ponderosa Pine, White Pine, and Douglas Fir, RMD 


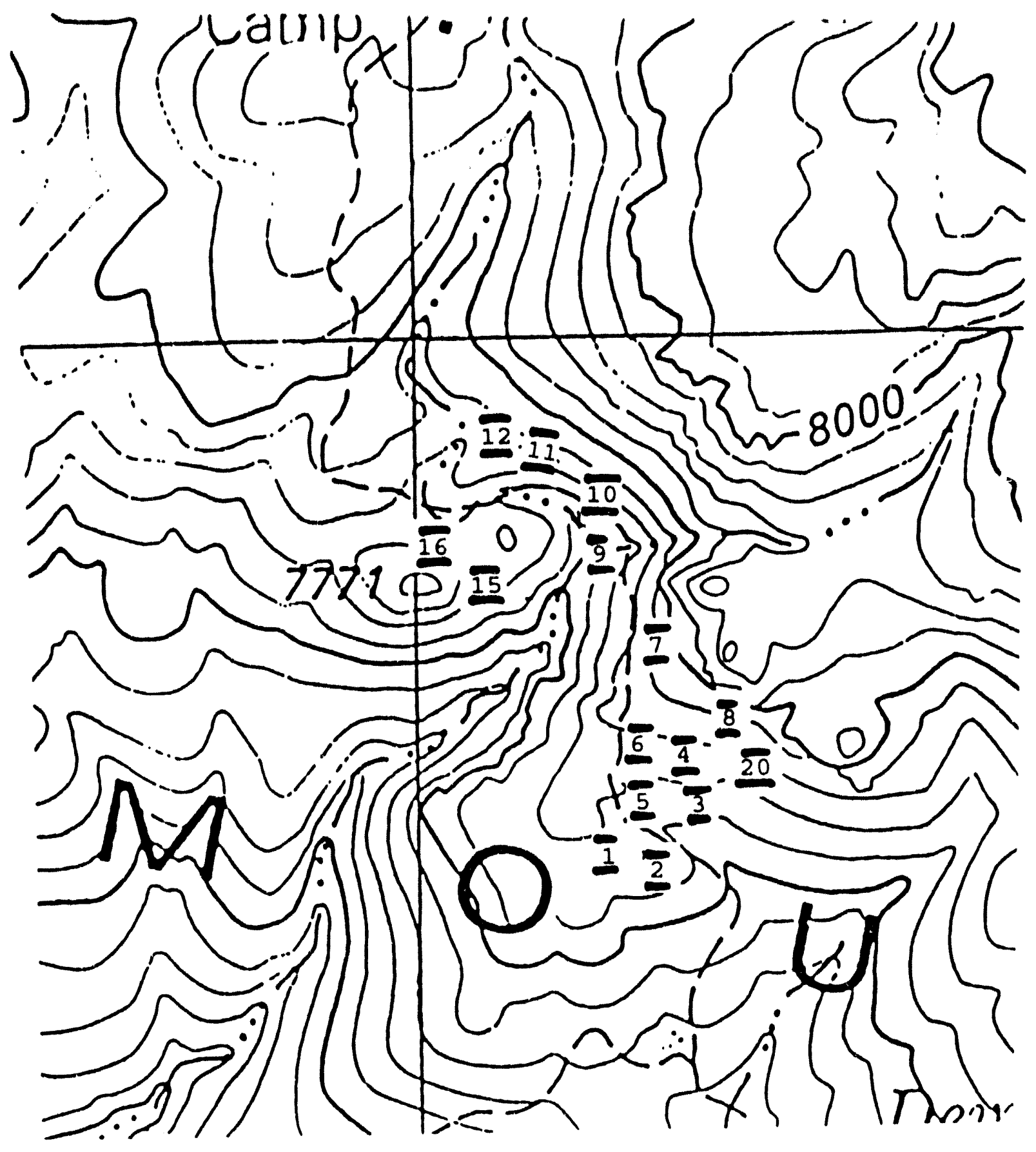

Figure A-13. Details of sampling sites in Tucson Side, Ponderosa and White Pine, RMI) 


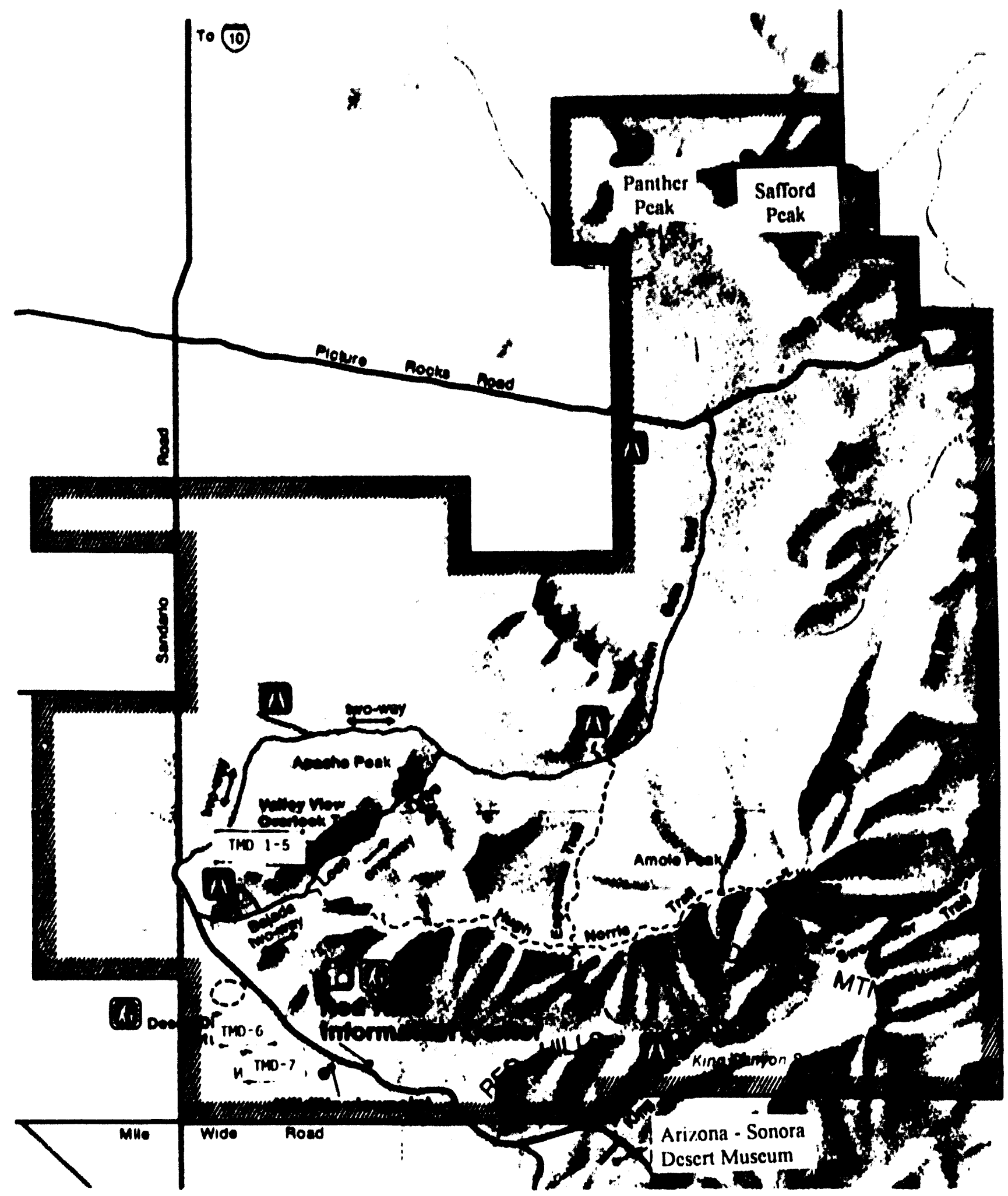

Figure A-14. Sampling sites in Tucson Mountain District, Saguaro National Monument (West). 
Appendix B Flemental Concentrations in Soil Quality Assurance Materials 


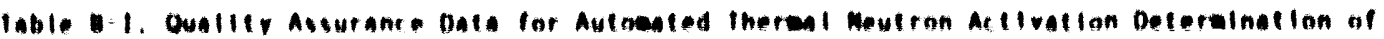

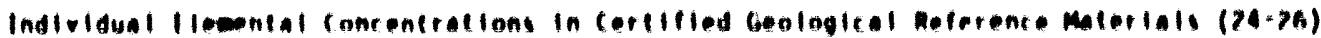

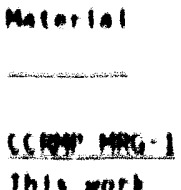

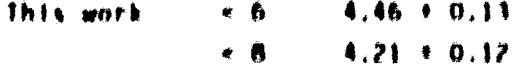

cerilliod $0.11 \cdot 0.01+.46 \cdot 0.15$

$$
\begin{array}{cc}
1.1 & +100 \\
+1.51 .1 .7 .5 & \cdot 600 \\
0.11 \cdot 0.110 .4 & 61.71
\end{array}
$$

$$
\begin{array}{r}
7.5 \\
+7.0 \\
\ldots .
\end{array}
$$

$11.0 \cdot 0.5$

$10.1 \cdot 0.4$

$10.5 \cdot 0.7$
$26 \cdot 7 \quad 160 \cdot 10$
$76 \cdot 1$
$76 \cdot 4 \cdot 167 \cdot 72$

1) 2104

thl wort

$*$

$0.15 \cdot 0.16$

- 1

$0.75,0.16$

cortited

$0.11 \cdot 0.16$

$71.1 \cdot 2.7$

$73.1 \cdot 7.4$

$+0$

$195 \cdot 41$

71.4 .0 .6

$+$

$100 \div 60$

$411+12$

$6.1 \cdot 0.17 .51 \cdot 0.14$

$6.6 \cdot 0.07 .57 \cdot 0.11$

$1.0 \quad 7.60,0.01$

$68 \cdot 109 \cdot 16$

64. $127 \cdot 16$

$17 \quad 100$

\section{1646}

inis wort

$\cos 11100$

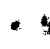

4

$-4$

$0.70 \cdot 0.11$

$0.02 \cdot 0.11$

5. As 0.11

$0.0 \mathrm{~ns}$

$6.75 \cdot 0.70$
$10.5 \cdot 1$.

$11.0 \cdot 1.1$

$10.6 \cdot 1.7$

$17 \cdot 1$

$0.10 \quad 0.24$

$9.18 \cdot 0.05$

in 1 .

certifled

$$
+5
$$

$x *$

$$
\begin{gathered}
7.110 .4 \\
7.5
\end{gathered}
$$

$+17$

- 11

- I1

a*
$116 \cdot 44$

$170 \cdot 50$

450.70

409
$191 \cdot 16$

$119 \cdot 17$

$171 \cdot 11$

III
$0.00,0.01$

$0.00 \cdot 0.01$

$0.66 \cdot 0.06$

$0.01 \cdot 0.01$
I6 $1,300,000$ $11 \cdot 11100 \cdot 900$

$60 \cdot 4$

13000
$16 \cdot 17900 \cdot 900$

Nos 164

Ihls work cerilifed

$1.7 \cdot 0.47 .45 \cdot 0.01$ $1.137 .76 \cdot 0.04$

CCAP $50-1$

Ints wort

certilled
- 6

$9.20 \cdot 0.10$

- 4

$0.04,0.25$

0.1

$9.16 \cdot 0.11$
$69 \cdot 0$

a) 1

$7.3 \div 0$.

1.9 .0 .4
7.0 .0
$+11$

7.1
$151 \cdot 10$

$191 \cdot 17$
.7 .1

$6.10 \cdot 0.11$

$11+1 \quad 100$

0.76

A. $4,0.16$
$11,213.9 \cdot 2.6$

\section{CCOA: $50-2$}

Ints wot

- J

$1.11 \cdot 0.27$

$1.6 \cdot 0.4 \cdot 12$

$1.6 \cdot 0.4 \times 15$

a.7

- 10

$<2.1$

- 10

< 1

<?

$0.01 \div 0.22$

Cerllled

0.19

8.01 0.18
-

$100 \cdot 40$

$1 / 26$
$1.6 \cdot 0$

$+\infty$
900.90

910.60

819:4

\section{$4.31 .67 \cdot 0.10$ $7.0,0.41 .11 \cdot 0.11$}

7.7

$1.00 \cdot 0.01$
$1020 \cdot 60$ $1050+60$ $10 \% 0+60$ $1020 \cdot 80$ 1000,10 $966 \cdot 67$
10.?

$$
16 \cdot ?
$$

$$
16+7
$$

$$
11.2
$$

$11 \cdot 2$

11.2
$110 \cdot 5$

$109 \cdot 3$

$102 \cdot 10$
$116 \cdot 25$

- 130

150
CCBap $50 \cdot 3$

Inls work

$1.11 \cdot 0.09$

1.11 .0 .13
$1.85 \cdot 0.11$

$$
1.61 \cdot 0.10
$$

$1.90 \cdot 0.10$

$1 . \omega 6 \cdot 0.10$

$1.13 \cdot 0.10$

$1.96 \cdot 0.10$
$111 \cdot 5111 \cdot 71$

$119 \cdot 5117,77$

$1115 \cdot 110$

$1115 \quad 100$

\begin{tabular}{|c|c|c|}
\hline $\begin{array}{r}2.5 \\
\end{array}$ & 5.45 & 10.16 \\
\hline$* 3$ & 5.19 & .0 .16 \\
\hline$<2.6$ & 5.59 & +0.15 \\
\hline 4.2 & 3.15 & 0.15 \\
\hline 0.17 & 5.46 & 10.14 \\
\hline
\end{tabular}

$123,6,136,20$

$112 \cdot 6$

84

$$
-2.0
$$

1.07. 0.09

certifled

1.06:0.11

\section{$7.6 \div 0.4 \div$ \\ $2.1 \cdot 0.3 \div 8$}

$2.51 \cdot 0.130 .99$
$370 \cdot 10$
$310 \cdot 10$
$300 \cdot 10$

$5.1,0$. $.7 \cdot 0.6$ 5.5
CCnes SO-4

Ihls work

$1.6 \cdot 0.8$

I,0 0 ,

$1.1 * 0.8$

$1.1 \cdot 0.8$

- 10

$110 \cdot 50$

$5.1+0.11 .08+0.01$

A00 $: 50$

$120 \div 10$

$1.7 \cdot 0.8 \quad 1.15 \cdot 0.01$

$6.0 \cdot 0.1 \quad 0.1$

$160 \times 40$

$0.6+0.6 \quad 1.01,0.01$

$1.4 \cdot 0.20 .64$
$100 \cdot 40$ 
table - 1. Oublity Asurance Deth for Automated therent Meutron Artivation Detereinatlon of

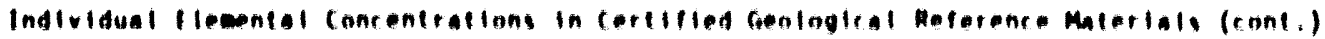

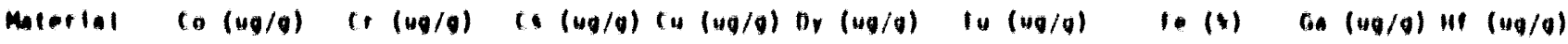

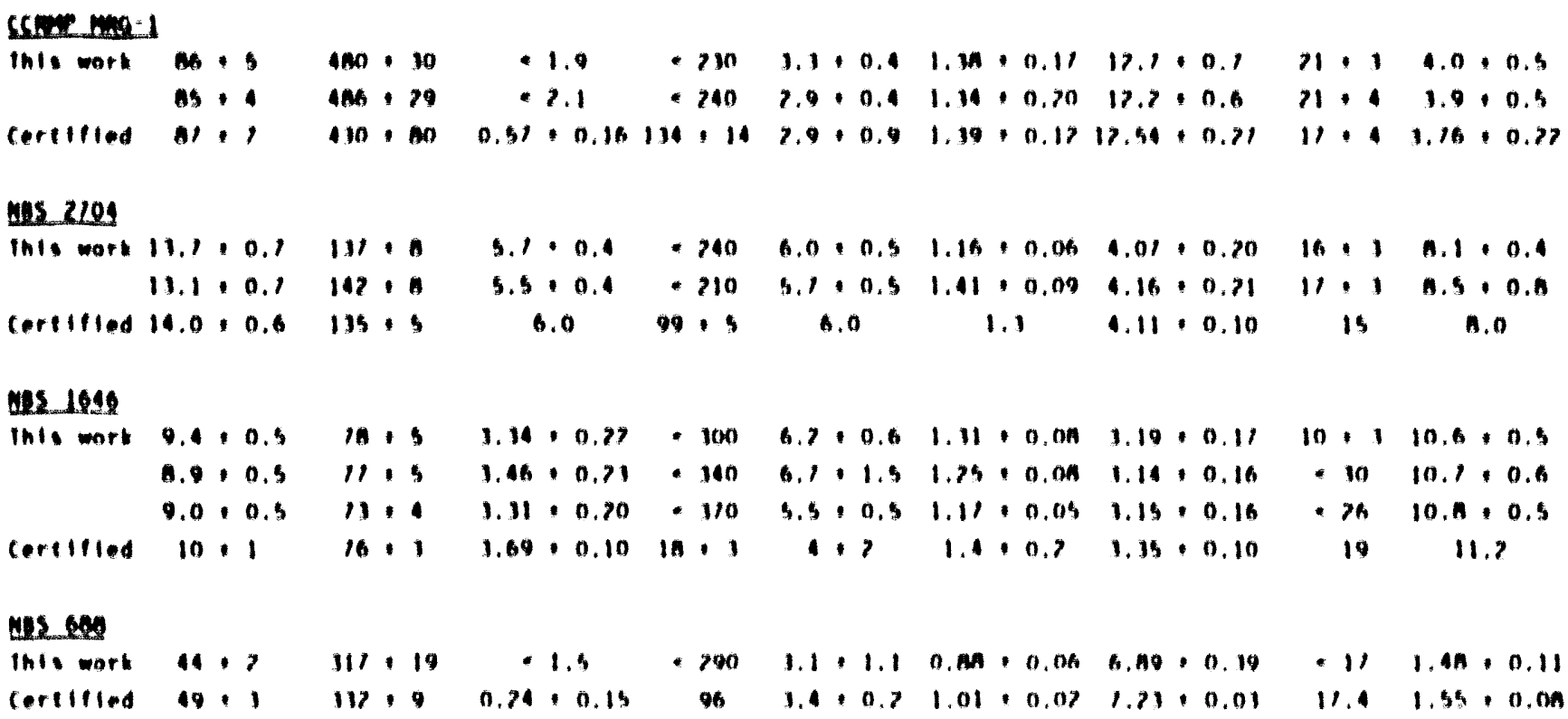

\section{wes 1645}

Ints wort $0.0,0.476100,17007.44,0.71770,60$

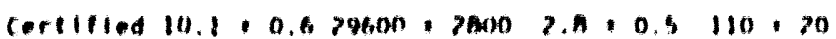

$*$

7.0
$0.17 \cdot 0.0410 .4 \cdot 0.5$

0.5

$11 \cdot 1$

$11.31 \cdot 0.16$

11.701 .19

CSer: $50^{\circ}$

Inl w wort

$76 \cdot 1$

fertilied 17,1
$170 \cdot 10$

$111 \cdot 10$

160.15
$4.1 \cdot 0.1 \cdot 170$

$4.6 \cdot 0.1 \cdot 100$

$5.01 \cdot 0.1601 .1$
$4.5 \cdot 0.41 .44 \cdot 0.07$

$1.0,0.41 .57 \cdot 0.0 \mathrm{~h}$

$1.5 \cdot 0.41 .31 \cdot 0.05$ a.6.0.1

$4.4,0.1$

$6,00 \cdot 0.11$
$71,3.5 \cdot 0.4$

$1,6,2.5,0.1$

$24,17.5,0.4$
SCR: $50:$ ?

InI wort $1.2,0.417 .9,1.10 .17,0.09,260$

$1.1 \cdot 0.413 .0 \cdot 1.10 .11 \cdot 0.09 * 100$

$1.7 \cdot 0.411 .6,1.1 * 1.790$

$1.4 \cdot 0.417 .0 \cdot 1.0 * 0.9 * 300$

$6.9 \cdot 0.111 .1 \cdot 1.0 \cdot 1.1 .100$

certifled $1.6,0.617 .3,1.30 .41,0.05,1,1$ a.) $10 ., 1.36 \cdot 0.10$ $0.7,0.1,1.70 \cdot 0.70$ $9.1,0.0,1.19,0.11$ $0.7 \cdot 0.1,1.24 \cdot 0.18$ $10.0 \cdot 1.0 \quad 1.10 \cdot 0.20$

A.6.1.1.15.0.06 $5.1 \cdot 0.1$ 5.) 0.1 $0.39 \cdot 0.79$ $5.10 * 0.21$ $3.36 \cdot 0.16$
$75 \cdot 170: 7$

2n:4 $20: 7$

$19 \cdot 470 \cdot 7$

$71,419.1,1.1$ $73,410.0,1.3$ $21,111.1 .1$

\section{CCBN: $50=3$}

Ints wort $5.6,0.3$

$5.5,0.3$

$2 A, 7$

$1.71,0.09$

$7) \div$

certified $5.5,0.6$
$1.01: 0.0$ a

- 700

110

$1.17 \cdot 0.0911 \cdot 1$
$2.8 \cdot 0.30 .11 \cdot 0.04 \quad 1.67,0.00$ $2.1,0.10 .67,0.04 \quad 1.67,0.09$

$2.0 .30 .14 \cdot 0.04,41,0.06$

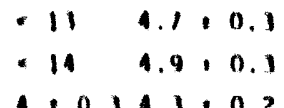

$.114 .1 \cdot 0.1$ $0.4 \cdot 0.1+1 \cdot 0.7$

ccoup so:

inls work $9.1,0.5$

ค. $\div 0.5$

$9.7 \cdot 0.5$

$10.0 * 0.5$

certilled $10.4,1.4$
$64 \cdot 4,2.16 \cdot 0.27 \times 160$

$11 \cdot 4$

66. 4

on 1

$61 \cdot 6$
$2.17 \cdot 0.16$

$2.16 \cdot 0.13$

$7.34,0.16$

$2.4 \cdot 0.19 \quad 27 \cdot 1$
$1.9+0.11 .00+0.012 .29 \cdot 0.17$ $4.7,0.60 .99,0.06,2.10,0.17$ $3.8,0.40 .0 A, 0.05,2.40,0.12$ $1.5,0.10 .89,0.04$ $1.5,0.60 .91 \cdot 0.01$

$0.25 \cdot 0.11$
- I1 a.1. 0.6 $11 \cdot 4$ I.6, 0.A $14,1,1.5,0.4$ A? $1 . \mathrm{H}, 0.4$ 


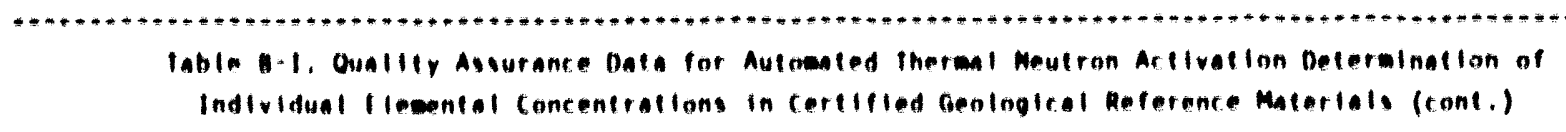

Indlaldual lleental toncentratlons in Certliled oeologlcel Reference Materlals (tont.)

\begin{tabular}{|c|c|c|c|c|c|c|c|c|c|}
\hline matertal & $110(n g / 0)$ & $1(40 / \theta)$ & $\ln (n 0 / 0)$ & $x(x)$ & I. (ug/g) & Iu $(n g / g)$ & $M(v)$ & $\operatorname{Mn}(u g / 0)$ & Na $(0)$ \\
\hline \multicolumn{10}{|c|}{ SCRAP 1} \\
\hline thls wort & $\begin{array}{r}1400 \\
+1000\end{array}$ & $\begin{array}{r}+74 \\
+72\end{array}$ & $\begin{array}{r}+270 \\
* 720\end{array}$ & $\begin{array}{l}0.11: 0.07 \\
0.16 \cdot 0.01\end{array}$ & $\begin{array}{l}9.3 \cdot 0.5 \\
9.6 \cdot 0.5\end{array}$ & $\begin{array}{r}114 \cdot 70 \\
95 \cdot 77\end{array}$ & $\begin{array}{l}1.16: 0.44 \\
1.10: 0.40\end{array}$ & $\begin{array}{l}1400 \cdot 60 \\
1110 \cdot 50\end{array}$ & $\begin{array}{l}0.545: 0.023 \\
0.536: 0.074\end{array}$ \\
\hline certillied & $140 \cdot 10$ & $\ldots$ & - $\$ 00$ & $0.15: 0.07$ & $9.6 \cdot 0.6$ & $120 \cdot 10$ & $0.17 \cdot 0.19$ & $1110 \cdot 100$ & $0.55 \div 0.06$ \\
\hline
\end{tabular}

\section{Ans 2109}

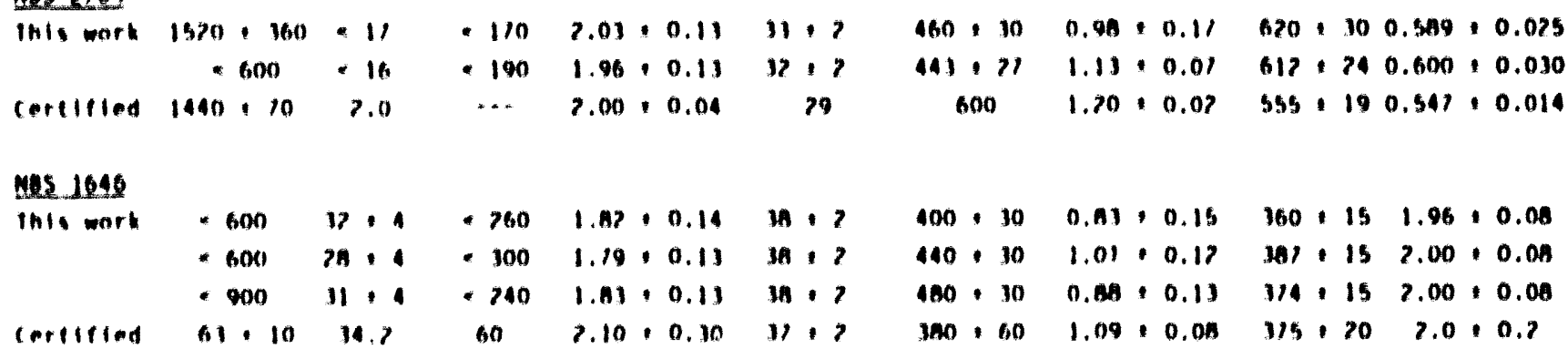

\section{N45. 64}

thls work 0 ono $* 19 \div 2100.130,0.0114 .1,0.1$

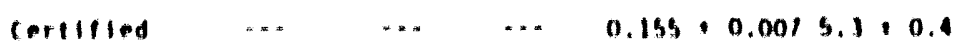

$110 \cdot 73$

$1.69 \cdot 0.19$

$350 \cdot 10$

$5.26 \cdot 0.27$

$1110 \div 60 \quad 1.56 \div 0.01$

N85 1645

Inl. wor

cercilled

$+1100 \cdot 14 \quad 720 \cdot 10$

$1.11 \cdot 0.09 \cdot 0.1 \cdot 0.3$

- 100

$0.58 \cdot 0.05$

$1290 \cdot 20 \quad 1.60: 0.02$

ccoup $50 \cdot 1$

\begin{tabular}{|c|c|c|c|c|c|}
\hline this wot & $\begin{array}{r}+1000 \\
+600\end{array}$ & $\begin{array}{l}=24 \\
=21\end{array}$ & $\begin{array}{r}.300 \\
.300\end{array}$ & $\begin{array}{l}2.60: 0.11 \\
2.16: 0.16\end{array}$ & $\begin{array}{l}35: 1 \\
51 \cdot 1\end{array}$ \\
\hline certilled & $27 \cdot 1$ & 17 & 100 & $7.68 \cdot 0.08$ & $34: ?$ \\
\hline
\end{tabular}

CCoup 50:?

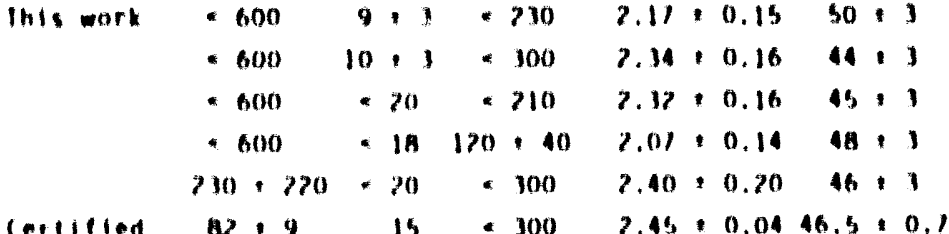

ienisted

cowis 50.

Inis wort

intilled

$\begin{array}{ccc}* 100 & * 11 & * 110 \\ * 100 & * 11 & * 140 \\ 11,1 & 1,0 & 100\end{array}$

$1.21 \cdot 0.0 \mathrm{~B} 11.2 \cdot 1.1$

$1.19 \cdot 0.0816 .9,0.9$

$1.16 * 0.0516 .9 \cdot 1.3$

ccoup 50 - 4

\begin{tabular}{|c|c|c|c|}
\hline \multirow{4}{*}{ inis work } & . 400 & -15 & - 160 \\
\hline & $\leftarrow 5,00$ & $* 16$ & $=180$ \\
\hline & $<1 / 0$ & .14 & .200 \\
\hline & $\times 100$ & -11 & .180 \\
\hline Centilled & $17 \cdot 10$ & 1.0 & 100 \\
\hline
\end{tabular}

$1.64 * 0.1128 .1 \cdot 1.6$

$1.11 \cdot 0.1121 .9 \cdot 1.1$

$1.85 \cdot 0.1321 .5 \cdot 1.4$

$1.85 \cdot 0.1329 .1 \cdot 1.1$

$1.13 \times 0.0128 .2 \cdot 1.1$

$158 \cdot 24$

$7.05 \cdot 0.16$

$2.01 \cdot 0.13$

$310 \cdot 20$

$2.31 \cdot 0.11$

539 11

525,30

sno: 10

$560 \cdot 30$

$310 \cdot 10$

$460+60$

$267 \cdot 15$

$261 \cdot 16$

$4.94 \cdot 0.73$

$1.00+0.30$

730,30

$4.9 n \cdot 0.10$

$511,730.15 \cdot 0.03$

$510,300.13 \cdot 0.03$

$520 \div 200.14: 0.04$

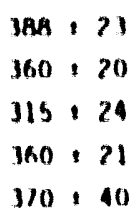

$0.33: 0.06$ $0.56: 0.06$ $0.45: 0.05$ $0.5 \mathrm{~s}, 0.06$ $0.36+0.04$ 


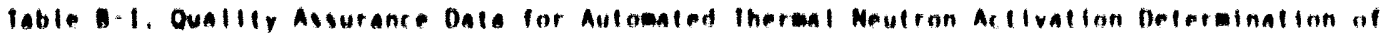

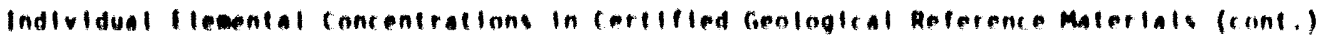

scher meis

inls work

$\begin{array}{ll}* 50 & +20 \\ 71.6 & .18\end{array}$

$410 \cdot 60$

$44 \cdot 1$

* I6

$4.4 \cdot 0.7 \quad .100$

IAD 100

certified

$19.76 .3,7.4$

$660 \cdot 160 \quad 35 \cdot 5$

$0.19,0.01$

$4.40 .2 * 110$

$170 \cdot 100$

Nos 2104

IIII) work

$29 \cdot 1$

$114 \cdot 1$

$3120+7 \mathrm{r}$

$11.6 \cdot 0.6$
$7.7 \cdot 0.1$
4

A.6.0.

$+750$

910.90

- $20 \quad 101 \cdot 6$

1650,100

$11.1,0.6$

$6.5+0.1+760$

940. 40

certilied

$100 \quad 1190 \cdot 150$

12

1.1

6. 1

110

\section{uns 1646}

inls wet

$31,65,5$

- 310

$10.1 \cdot 0.5$

$0.5 \cdot 0.4$

$6.8+0.1 \cdot 400$

$1110 \cdot 90$ $40 \cdot 197 \cdot 6$

$450 \cdot 170$

$10.3+0.6$

$0.9 \cdot 0.1$

$6.1+0.1 \quad+740$

$910 \cdot$ an

4), 85,5

$310 \cdot 110$

$10.0 \cdot 0.5$

$1.8 \cdot 0.6$

$1.1 \cdot 0.1 \times 110$

$980 \cdot B 0$

1000

cerilled

B) 100,200

$10.8 \cdot 0.4$

$0.51 \cdot 0.09$

$6.4 .0 .1 \quad 720$

\section{M⿻5 698}

Ints work

410

ceriflied

$9.6 \cdot 1,90 \cdot 0.01300,200$

$34: ?$
$16: ?$

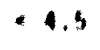

$2.7 \cdot 0.1$

* 310

600

1

$2.5 \cdot 0.2$

$169 \cdot 1$

$110 \cdot 10$

\section{NeS 1645}

inis work

cercllled

- 16 43:4 346000, 11000

$2.0 \cdot 0.1$

- 1

8.0

$1.1+0.4$

$1.75,0.06$

$140: 90$

$-300$

... $11,4310000,60000$

CCBup SO-1

inls work

3) A $|2| \cdot 1$

$+100$

$35: 9174,1$

430

$16.8 \cdot 0.9$

4

$16.4,0.9$

- 5

$11.1 \cdot 0.80 .091 \cdot 0.001$

ค. $3: 0$.

A. 0,0 .

$110 \cdot 90$

- 110

$610 \cdot 10$

cercilled

$44,139+17 \quad 291,25$

$1.9 \cdot 0.5$

$12 A+29$

$100: 120$

CLOAP 50-?

ints work

64.

$66 \cdot 4$

$+400$

$60 \div$

(), 4

$12+8$

$14 \cdot 5$

$60,111 \cdot 3$

$36 \div 6 \quad 11 \cdot 5$

cerified

18:6

CCRAP 50-3

Ints work

$79 \cdot 4$

40,1

$350 \cdot 60$

$+200$

$10.1 \cdot 0.6 \quad 1.9 \cdot 1.7$

$17.1+0.6$

e 0.1

$11.4 \cdot 0.5$

$1340 \cdot 100$

- 100

$10.8 \cdot 0.6$

0.4

$11.4,0.5$

430,90

$1100 \cdot 100$

- 140

11.0 .0 .6

- 0.3

$14.6,0.1$

390,60

$1180 \cdot 120$

- 140

$11.0 \cdot 0.6 \quad 0.3$

$11.1,0.5$

$160 \cdot 100$

$1190: 100$

$110+20$

$11.1: 0.3$

$0.40 \div 0.08$

$11.8 \cdot 1.2$

- 2a0

$1770 \cdot 90$

cerellied

$21 \cdot 5$

$35 \cdot 2$

4.0

$+3$

$1.30 \cdot 0.15=230$

$110 \cdot 10$

11,1

$19 \cdot 3$

$32) 6$

5.; 0.1

$+2.4$

$3,37 \cdot 0,15 \times 100$

360,30

$5.2 \cdot 0.30 .034 \cdot 0.015$

$3.41 \cdot 0.19720: 30$

430.60

CCROP SO-4

Ints wark

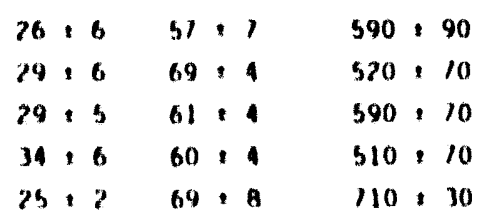

6.0.0.4

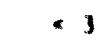

$1.8 * 0.4$

$1.5 \cdot 0.4$

$$
<1
$$

$0.83+0.34$

8. $3,0.4$

$+0.9$

$4.60 \cdot 0.2$

* 270

$610+10$

8. 0.4

$0.49 \cdot 0.01$

$4.86+0.2$

$+300$

$600+60$

Certllied 


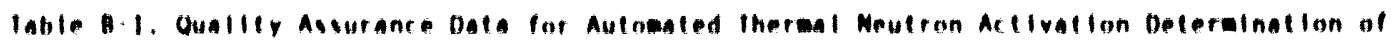
Individual I Iomental Concentrations In Cortilled Geologleal hoterence Materlals (cont.)

Mateital if (nq/g) in $(40 / 9)$ if $(40 / 9)$

SCH: MRO

Ints wort $100 \cdot 100.86 \cdot 0.1172000 \cdot 1000$ $100 \cdot 1100.14 \cdot 0.1021800 \cdot 7800$

Certifled $310,600.93,0.1872600,000$

NaS 2109

Int wort

B20 - Bn

$9.1,0.4$

$1100 \cdot 600$

Q4O + BO

$9.1 \cdot 0.4$

0.7

$4600+600$

(ertilled

...

$45 / 0 \cdot 180$

$1730 \cdot 120$

$1070 \cdot 120$

$1110 \cdot 110$

$99 \cdot 47.7 \cdot 0.41 .1 \cdot 0.7$

$91+2.4+0.63 .5,0.2$

95.4 .6
$470 \cdot 50 \quad 190 \cdot 10$

$430 \cdot 60780 \cdot 80$

$138 * 17 \quad 300$
NBS 1616

Ints work

certiled

$110 \times 10$

610.80

130,10

950

$10.7 \cdot 0.4$

$9.4 * 0.4$

$9.7 \cdot 0.1$

$10.0 \cdot 0.6$

$4100+600$

1900,100

$1800+600$

1200,800

\section{NBS 689}

IhIs work

cerllled

$100 * 110$

$570+40$

$0.79,0.00$

$5800+800$

$1010 \cdot 60$

$168 \cdot 18$

$310 \cdot 24$

$715 \cdot 9$

$\ldots$
$\ldots$

$1 . \mathrm{AA} \cdot 0.15$

$7.05 \cdot 0.20$
$90 * 30600 \cdot 140$

$104 \cdot 21,400 \cdot 100$

$111 \cdot 15110 \cdot 60$

$116 \cdot 635$

MBS 1645

Ihtswork $\quad 4.14 \cdot 0.209 / 0 \cdot 180$

certified

$\ldots \quad 1.6,0.7 \quad 500+200$

$1100: 110$

$21 \cdot 21.1,0.40 .68 \cdot 0.10$

- $16 \quad 710$

$84+1060.6,1.9$

C CBN: SO-1

this wort

$180 \cdot 80 \quad 11.2 \cdot 0.45000 \cdot 100$

$850 \cdot 100 \quad 10.6 \cdot 0.94600 \cdot 600$

$1110 \cdot 50$

$24 \cdot 1$

44

0.6

$1540+190<160$

$12.4 \cdot 1.2$

$4,600 \cdot 600$

$1620+10$

117

$16 / 0 \cdot 70$

171

6
0,6

$2.15 \cdot 0.23$

$7.41 \cdot 0.70$

$1 / 10 \cdot 40$

111

).

$7.24 \cdot 0.24$

$120 \cdot 16$

$120 \cdot 10$

certlled

cckn so-?

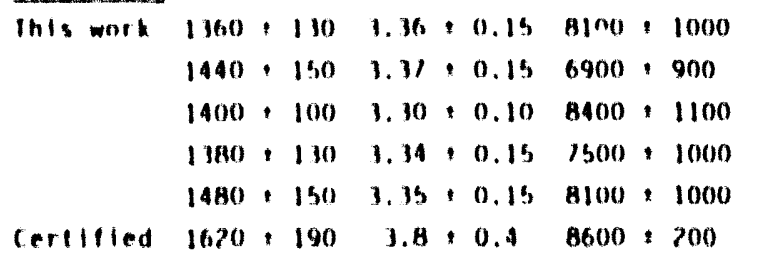

$968 \cdot 43$

$990 \cdot 40$

890: 40

930,40

$889+19$

980,50

$59 \cdot 1<5$

$35+1=5$

$50+1<5$

$56+1=5$

$50,1=0$

$5,40.4$
$4.0 \cdot 0.3$

$4.0: 0.1$

$1.96 \cdot 0.76$

$3.67+0.24$

$1.9 * 0.3$

$3.5: 0.4$

$146 \cdot 584 \cdot 10$

\section{CCMP 50-3}

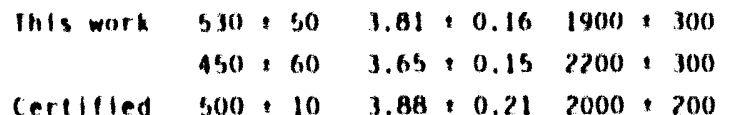

\section{COAI' SO-4}

lilis work

610,60
580,10
600,60
Certifled 610,30

$$
\begin{aligned}
& \text { 6. } 1 \cdot 0.3 \\
& \text { 1.8:0.3 }
\end{aligned}
$$

$1120+50$

$1100 \div 40$

$1110 \cdot 20$

$$
\begin{array}{r}
35+2 \times 3 \\
31+2 \times 3 \\
36+4
\end{array}
$$

$1.17 \cdot 0.12$

$1.59 \cdot 0.13$

$1.61 \cdot 0.18$
$115 \cdot 25,100,100$

$120 \cdot 161510,720$

$140+20800,100$

$100 \cdot 30950 \cdot 110$

$119: 16,120 \cdot 120$

$124: 5160,60$
$2140 \cdot 90$

2350,90

$7190+80$

$2320 \div 40$

$2380+10$

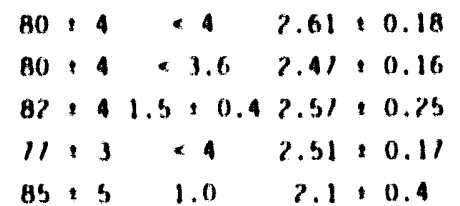

$58 \cdot 8 \quad 260 \cdot 50$ $55,1390,60$ $46.3 \cdot 1.8156 \cdot 13$
93 $12 \quad 300 \cdot 50$

$95 \cdot 17 \quad 250 * 40$

$85+11260+40$

$91 \cdot 12 \quad 560 \cdot 90$

$94+3 \quad 210+15$ 
Appendix C: Elemental Concentrations in Soils

$-39-$ 


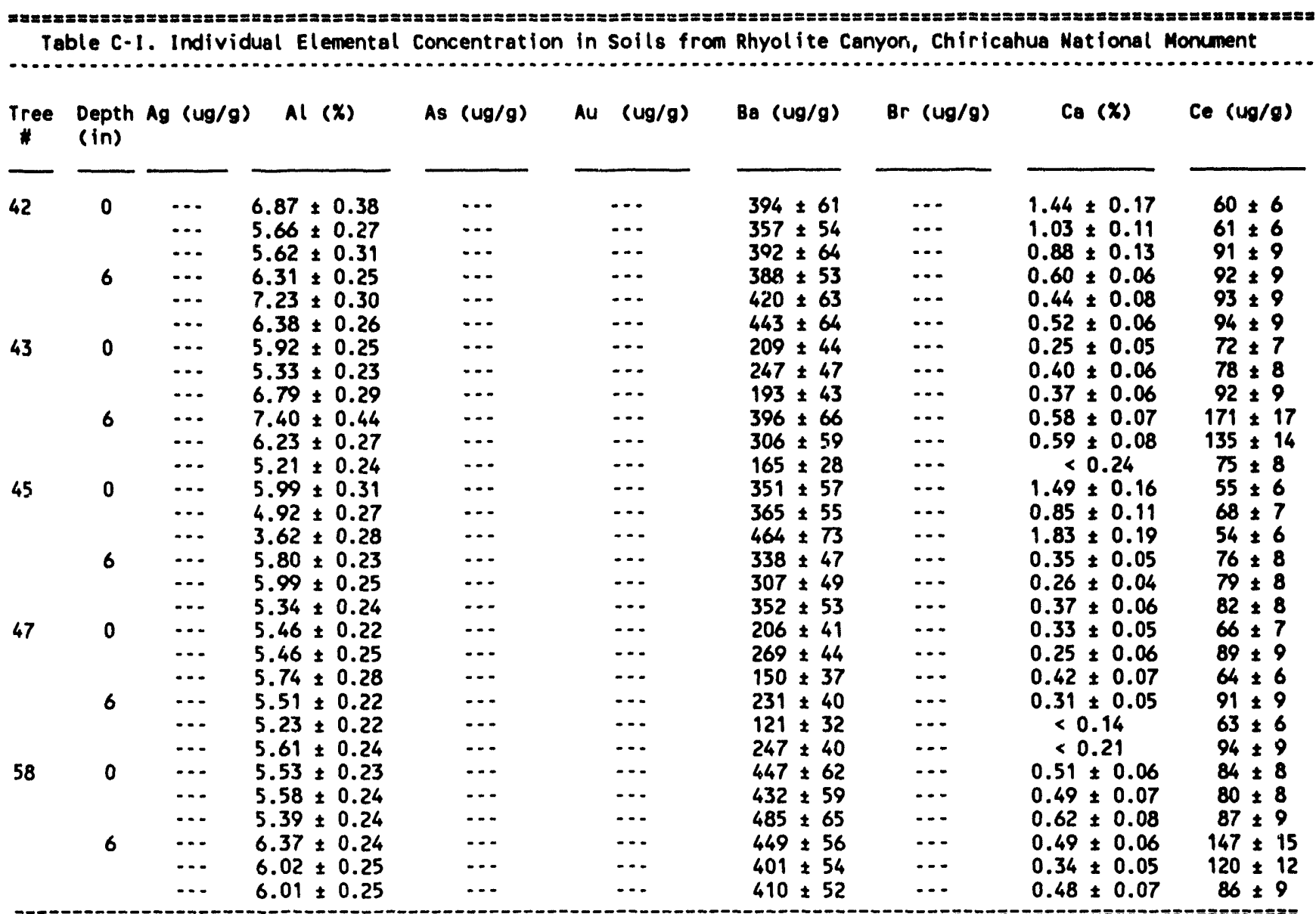

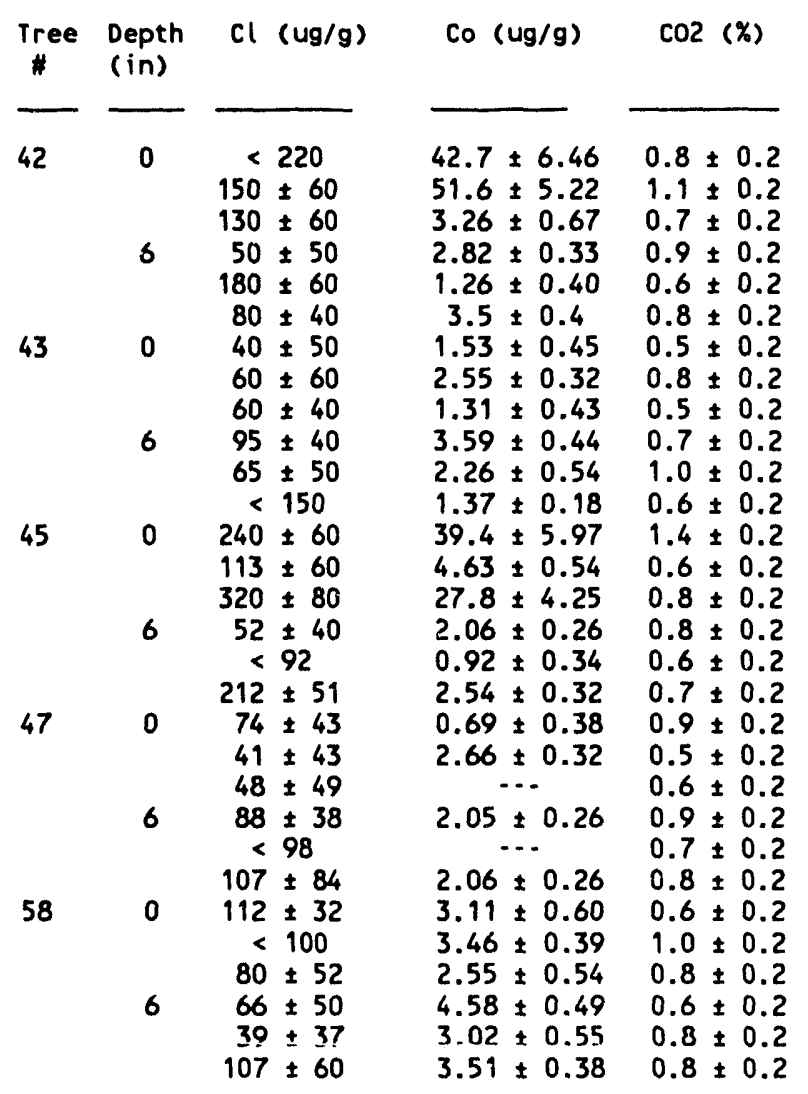
-

$80.3 \pm 8.27$ $91.7 \pm 6.63$ $22.5 \pm 2.6$ $25.1 \pm 2.08$ $22.2 \pm 2.47$ $25.5 \pm 2.16$ $15.2 \pm 2.0$ $13.6 \pm 1.35$ $15.0 \pm 1.9$ $23.7 \pm 2.09$ $15.7 \pm 1.94$ $7.76 \pm 0.88$ $77.4 \pm 7.96$ $23.0 \pm 2.02$ $62.5 \pm 6.55$ $17.0 \pm 1.43$ $15.9 \pm 1.87$ $22.0 \pm 1.81$ $9.46 \pm 1.3$ $14.6 \pm 1.38$ $8.82 \pm 1.25$ $11.2 \pm 1.15$ $5.43 \pm 0.99$ $12.4 \pm 1.28$ $26.1 \pm 2.9$ $24.4 \pm 1.96$ $20.6 \pm 2.32$ $28.7 \pm 2.27$ $23.4 \pm 2.54$ $22.9 \pm 1.82$

Dy (ug/g)

Eu (ug/g)

$6.0 \pm 0.6$ $5.73 \pm 0.62$ $6.91 \pm 0.62$ $7.44 \pm 0.78$ $7.68 \pm 0.67$ $7.77 \pm 0.82$ $5.49 \pm 0.50$ $5.14 \pm 0.57$ $5.23 \pm 0.47$ $7.01 \pm 0.75$ $7.05 \pm 0.63$ $4.31 \pm 0.46$ $5.83 \pm 0.54$ $6.39 \pm 0.69$ $5.19 \pm 0.51$ $7.14 \pm 0.74$ $6.67 \pm 0.57$ $5.99 \pm 0.64$ $4.68 \pm 0.44$ $5.4 \pm 0.6$ $5.01 \pm 0.46$ $5.06 \pm 0.54$ $3.81 \pm 0.36$ $5.53 \pm 0.59$ $6.1 \pm 0.6$ $6.0 \pm 0.6$ $6.87 \pm 0.60$ $7.41 \pm 0.77$ $7.4 \pm 0.6$ $3.39 \pm 0.67$
$4.0 \pm 0.7$

$4.5 \pm 0.7$

$8.0 \pm 0.9$

$6.2 \pm 0.6$

$6.6 \pm 0.6$ $<5.7$

$4.2 \pm 0.8$

$5.3 \pm 0.4$

$6.9 \pm 0.6$

$14.5 \pm 1.2$

$10.4 \pm 0.9$

$3.1 \pm 0.3$

$3.4 \pm 0.6$

$4.2 \pm 0.6$ $<11$

$4.3 \pm 0.4$

$5.2 \pm 0.6$

$5.5 \pm 0.5$

$4.4 \pm 0.5$

$6.1 \pm 0.6$

$4.1 \pm 0.5$

$7.4 \pm 0.7$

$4.7 \pm 0.4$

$7.0 \pm 0.6$

$8.0 \pm 0.8$

$6.1 \pm 0.6$

$5.8 \pm 0.6$

$9.0 \pm 0.8$

$8.0 \pm 0.6$

$7.8 \pm 0 . \overline{7}$
$0.513 \pm 0.079$

$0.422 \pm 0.066$

$0.661 \pm 0.083$

$0.599 \pm 0.078$

$0.544 \pm 0.070$

$0.575 \pm 0.079$

$0.31 \pm 0.05$

$0.309 \div 0.057$

$0.304 \neq 0.051$

$0.55 \neq 0.08$

$0.378 \pm 0.069$

$0.181 \pm 0.033$

$0.466 \pm 0.076$

$0.51 \div 0.08$

$0.506 \pm 0.086$

$0.346 \pm 0.057$

$0.367 \pm 0.051$

$0.455 \pm 0.067$

$0.194 \pm 0.046$

$0.336 \pm 0.055$

$0.369 \pm 0.056$

$0.271 \pm 0.048$

$0.202 \pm 0.041$

$0.283 \pm 0.049$

$0.675 \pm 0.079$

$0.543 \pm 0.071$

$0.587 \pm 0.071$

$0.606 \pm 0.076$

$0.511 \pm 0.061$

$0.6 \overline{4} \pm 0.074$ 


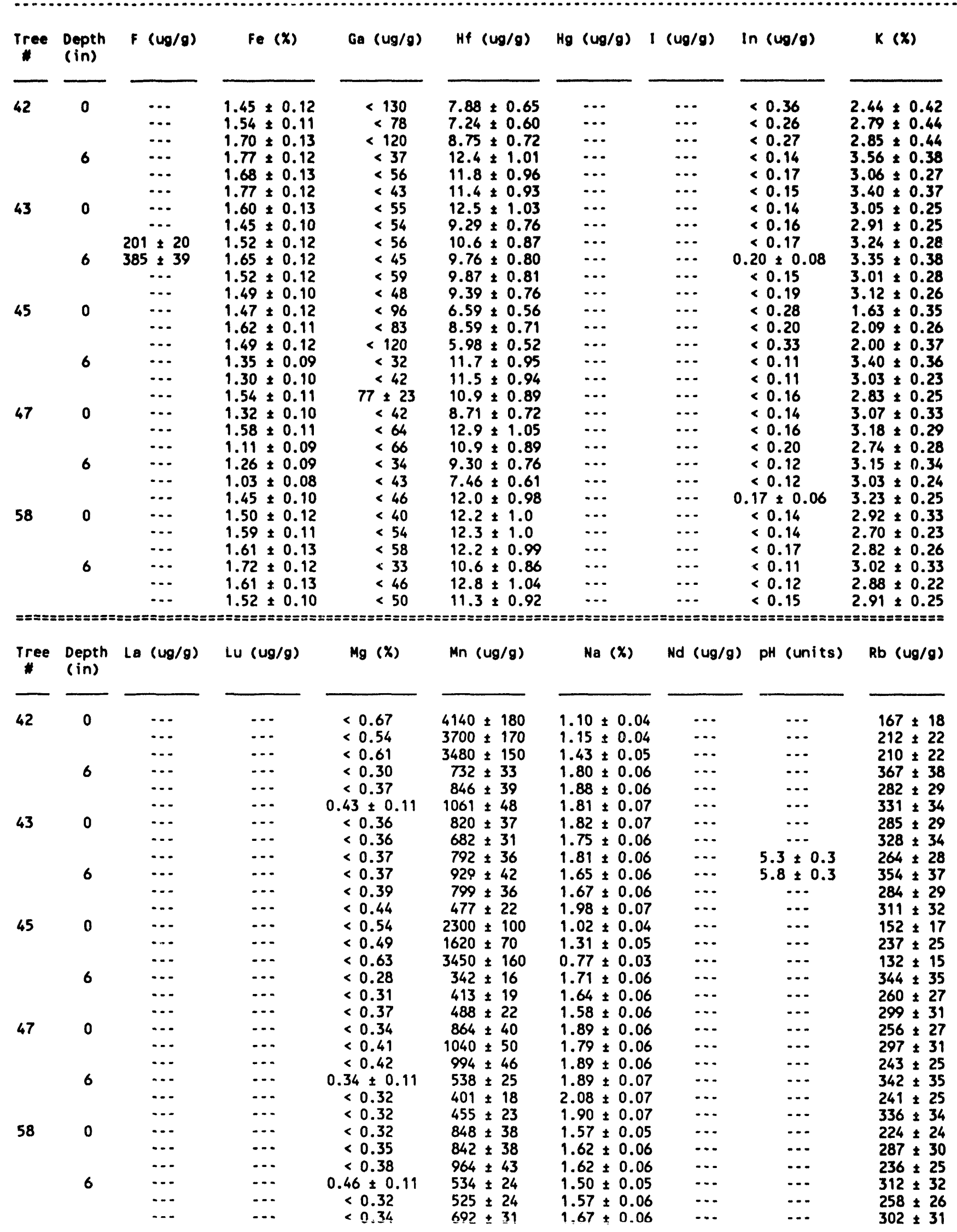




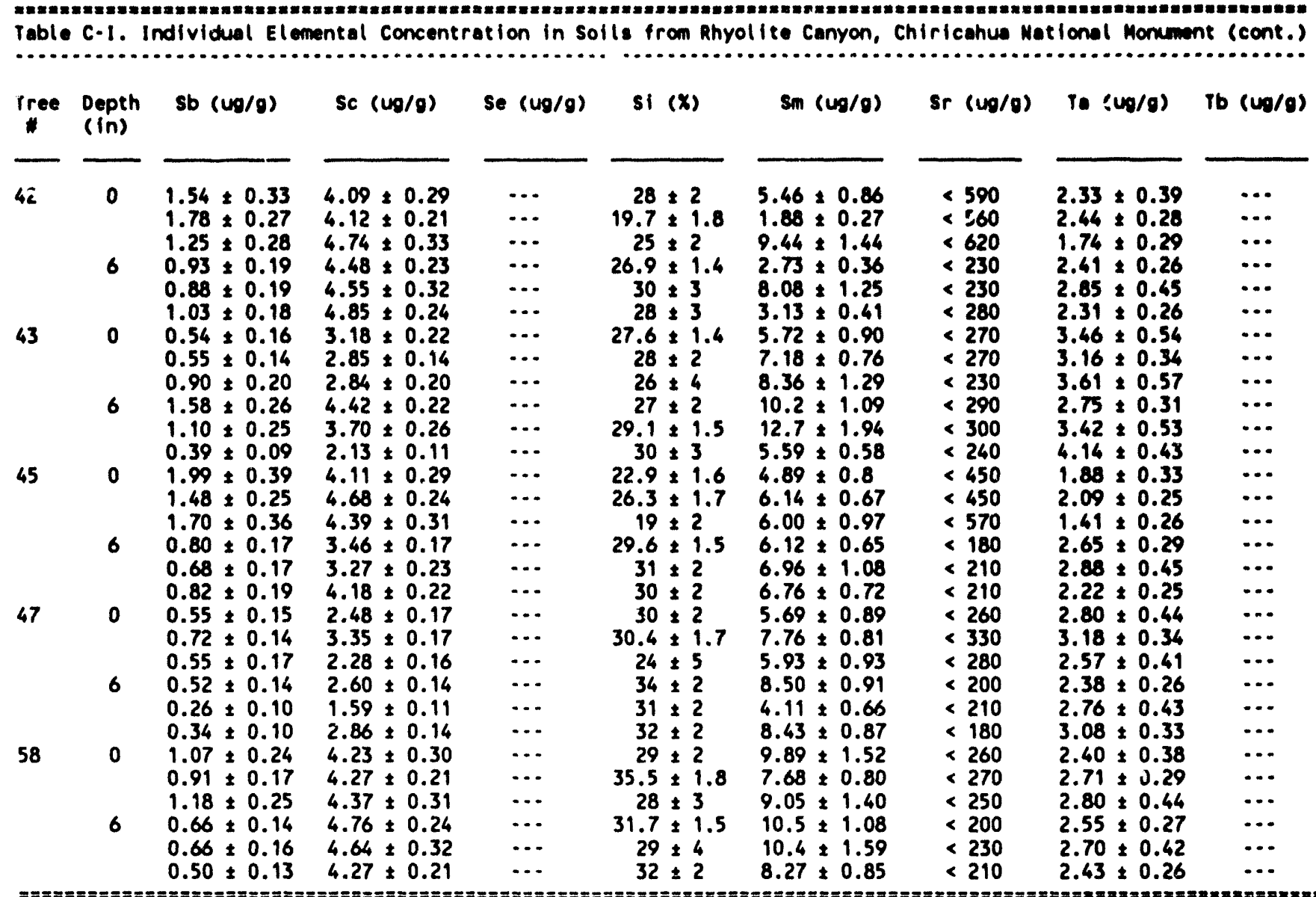

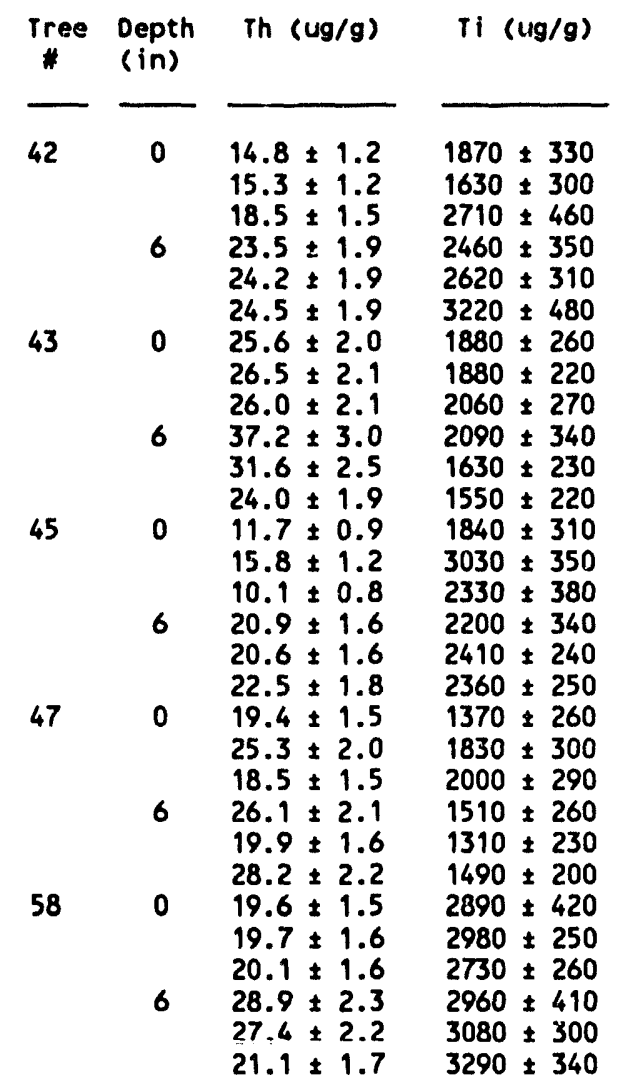

$U(u g / g)$

$v(u g / g)$

$H(u g / g)$

Yb (ug/g)

$\operatorname{Zn}(u g / g) \operatorname{2r}(u g / g)$

$3.73 \pm 0.3$

$3.89 \pm 0.30$

$4.92 \pm 0.40$

$5.48 \pm 0.45$

$5.64 \pm 0.40$

$5.37 \pm 0.40$

$5.55 \pm 0.40$

$5.70 \pm 0.45$

$5.87 \pm 0.50$

$7.54 \pm 0.60$

$6.29 \pm 0.50$

$5.28 \pm 0.40$

$3.46 \pm 0.30$

$4.82 \pm 0.40$

$2.73 \pm 0.25$

$6.73 \pm 0.60$

$6.93 \pm 0.60$

$5.89 \pm 0.50$

$5.11 \pm 0.40$

$6.09 \pm 0.50$

$5.24 \pm 0.40$

$6.55 \pm 0.55$

$5.30 \pm 0.45$

$6.28 \pm 0.50$

$6.4 \pm 0.5$

$5.95 \pm 0.50$

$5.98 \pm 0.50$

$7.36 \pm 0.60$

$6.84 \pm 0.60$

$6.51 \pm 0.60$
$24.9 \pm 3.1$

$28.0 \pm 2.4$

$30.1 \pm 2.7$

$22.6 \div 1.9$

$29.7 \pm 2.3$

$19.8 \pm 2.0$

$18.0 \pm 2.2$

$15.1 \pm 2.0$

$23.2 \pm 2.0$

$16.8 \pm 2.3$

$<6.1$

$26.4 \pm 2.7$

$31.9 \pm 2.8$

$28.3+2.7$

$19.0 \pm 1.9$

$14.6 \pm 1.8$

$21.5 \pm 2.4$

$11.0 \pm 1.5$

$17.6 \pm 2.3$

$16.8 \pm 2.6$

$13.7 \pm 1.7$

$6.9 \pm 1.4$

$14.1 \pm 2.0$

$36.4 \pm 2.6$

$32.0 \pm 2.5$

$29.4 \pm 2.7$

$37.2+2.5$

$28.3 \pm 2.3$

$29.1 \pm 2.4$
$23.6 \pm 2.3$
$370 \pm 100$

$400 \div 68$

$<280$

$<150$

$<140$

$<150$

$<130$

$<180$

$<140$

$<160$

$<140$

$<170$

$257 \pm 84$ $<200$

$300 \pm 110$ $<99$

$<93$

$<130$

$<140$

$<150$

$<180$

$<110$

$<94$

$<110$

$<140$

$<120$

$<150$

$<110$

$<110$

$<130$
$3.01 \pm 0.35$

$3.14 \pm 0.34$

$4.18 \pm 0.46$

$4.44 \pm 0.46$

$4.49 \pm 0.48$

$4.56 \pm 0.48$

$3.47 \pm 0.38$

$3.74 \pm 0.40$

$4.43 \pm 0.47$

$7.67 \pm 0.79$

$6.23 \div 0.65$

$3.09 \pm 0.32$

$2.41 \pm 0.31$

$3.03 \pm 0.35$

$2.45 \pm 0.31$

$3.73 \pm 0.39$

$3.70 \pm 0.40$

$3.75 \pm 0.39$

$3.16 \pm 0.35$

$4.46 \pm 0.46$

$3.36 \pm 0.37$

$4.74 \pm 0.49$

$2.93 \pm 0.31$

$4.68 \pm 0.49$

$5.03 \pm 0.53$

$4.05 \pm 0.42$

$4.28 \pm 0.45$

$5.37 \pm 0.55$

$5.60 \pm 0.58$

$4.49 \pm 0.46$

\begin{tabular}{rlll}
\hline 138 & \pm 28 & $\ldots$ \\
134 & \pm 20 & $\ldots$ \\
$116 \pm 24$ & $\ldots$ \\
$76 \pm 12$ & $\ldots$ \\
$89 \pm 18$ & $\ldots$ \\
$84 \pm 13$ & $\ldots$ \\
$94 \pm 19$ & $\ldots$ \\
$77 \pm 12$ & $\ldots$ \\
$78 \pm 16$ & $\ldots$ \\
$109 \pm 17$ & $\ldots$ \\
$90 \pm 18$ & $\ldots$ \\
$66 \pm 10$ & $\ldots$ \\
$109 \pm 22$ & $\ldots$ \\
$112 \pm 17$ & $\ldots$ \\
$115 \pm 23$ & $\ldots$ \\
$67 \pm 10$ & $\ldots$ \\
$56 \pm 11$ & $\ldots$ \\
$70 \pm 11$ & $\ldots$ \\
$71 \pm 14$ & $\ldots$ \\
$86 \pm 13$ & $\ldots$ \\
$56 \pm 11$ & $\ldots$ \\
$77 \pm 12$ & $\ldots$ \\
$49 \pm 10$ & $\ldots$ \\
$71 \pm 11$ & $\ldots$ \\
$88 \pm 18$ & $\ldots$ \\
$70 \pm 11$ & $\ldots$ \\
$67 \pm 13$ & $\ldots$ \\
$79 \pm 12$ & $\ldots$ \\
$65 \pm 13$ & $\ldots$ \\
$65 \pm 9$ & $\ldots$
\end{tabular}




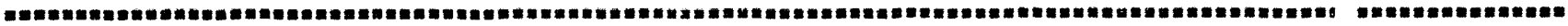
Toble C-i!. Individual Elemental Concentration In Solls from Gila Cliffe Mational Monument, Mew Mexico

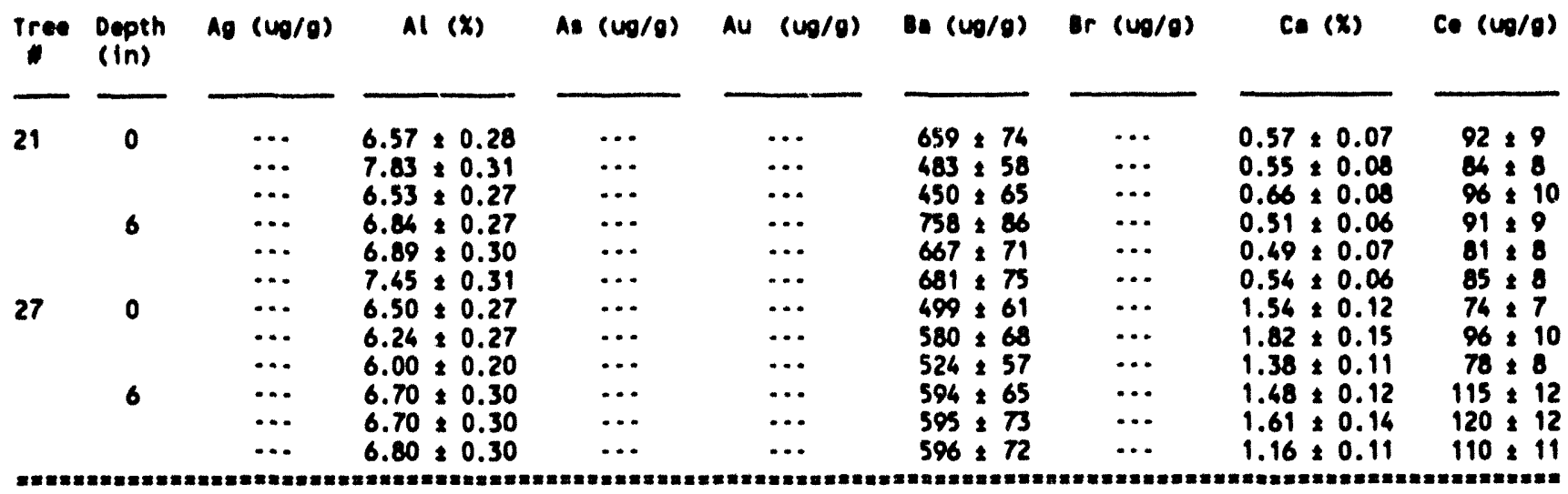

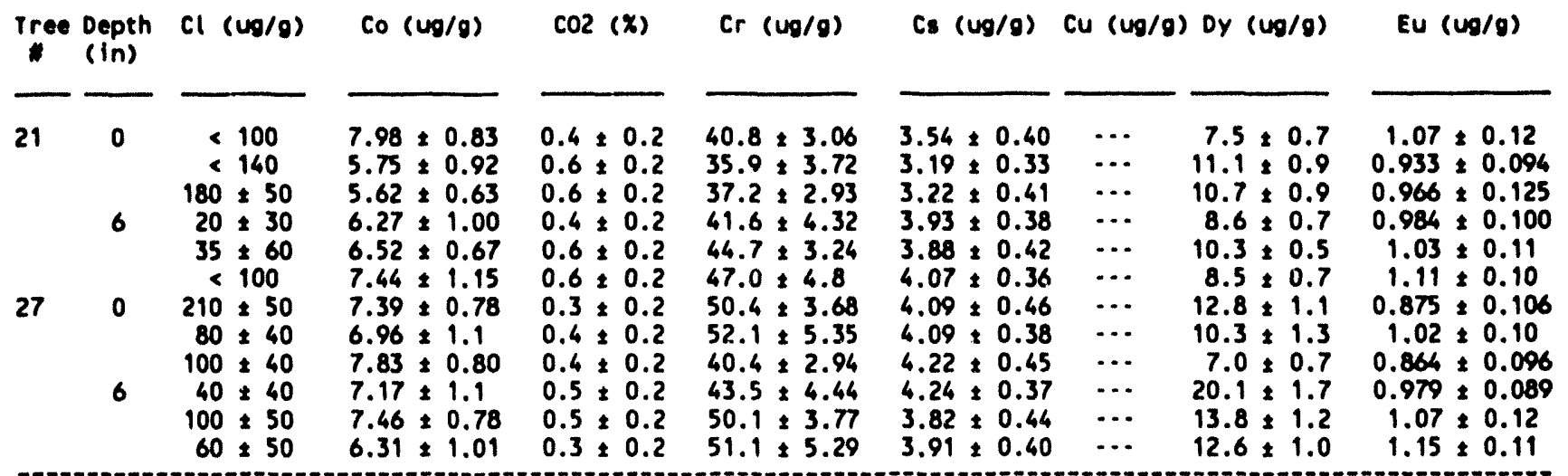

\begin{tabular}{|c|c|c|c|c|c|c|c|c|c|}
\hline Tree & $\begin{array}{l}\text { Depth } \\
\text { (in) }\end{array}$ & F $(10 \mathrm{~g} / g)$ & Fe $(x)$ & Go (ug/g) & Hf $(u g / g)$ & Hg (ug/g) & I (ug/g) & $\ln (\log / g)$ & $K(x)$ \\
\hline \multirow[t]{6}{*}{21} & 0 & $\cdots$ & $2.33 \neq 0.16$ & $<58$ & $10.6 \div 0.86$ & $\cdots$ & $\cdots$ & $<0.18$ & $3.63 \neq 0.31$ \\
\hline & & $\cdots$ & $2.40 \pm 0.19$ & $<58$ & $9.75 \div 0.79$ & $\ldots$ & $\ldots$ & $<0.18$ & $3.06 \neq 0.24$ \\
\hline & & $250 \div 25$ & $2.32 \times 0.16$ & $<45$ & $11.0 \div 0.90$ & $\cdots$ & $\cdots$ & $<0.16$ & $3.16 \pm 0.36$ \\
\hline & 6 & $352 \pm 35$ & $2.28 \pm 0.18$ & $<48$ & $10.4 \pm 0.85$ & $\cdots$ & $\cdots$ & $<0.12$ & $3.44 \div 0.27$ \\
\hline & & $\cdots$ & $2.26 \pm 0.16$ & $<54$ & $10.4 \pm 0.84$ & $\cdots$ & $\cdots$ & $<0.21$ & $3.47 \pm 0.30$ \\
\hline & & $\cdots$ & $2.45 \neq 0.19$ & $<54$ & $11.1=0.90$ & $\cdots$ & $\cdots$ & $<0.17$ & $3.63 \pm 0.29$ \\
\hline \multirow[t]{5}{*}{27} & 0 & $\cdots$ & $2.35 \pm 0.16$ & $<45$ & $10.0 \div 0.81$ & $\cdots$ & $\cdots$ & $<0.16$ & $3.01 \pm 0.35$ \\
\hline & & $\cdots$ & $2.68 \pm 0.21$ & $<59$ & $10.5 \div 0.86$ & $\cdots$ & $\cdots$ & $<0.15$ & $3.06 \div 0.27$ \\
\hline & 6 & $\ldots$ & $\begin{array}{l}2.09 \pm 0.14 \\
2.57 \div 0.20\end{array}$ & $\begin{array}{l}<40 \\
<50\end{array}$ & $7.82 \pm 0.63$ & $\cdots$ & $\cdots$ & $<0.13$ & $2.90 \pm 0.30$ \\
\hline & 0 & $\cdots$ & $\begin{array}{l}2.57 \pm 0.20 \\
2.54 \pm 0.18\end{array}$ & $\begin{array}{l}<50 \\
<60\end{array}$ & $\begin{array}{l}10.2 \pm 0.83 \\
10.9 \pm 0.89\end{array}$ & $\cdots$ & $\cdots$ & $\begin{array}{l}<0.14 \\
<0.20\end{array}$ & $\begin{array}{l}2.9 \neq 0.2 \\
3.0 \neq 0.3\end{array}$ \\
\hline & & $\cdots$ & $2.38 \neq 0.1$ & $53 \pm 18$ & $12.9 \pm 1.05$ & $\cdots$ & $\cdots$ & $<0.16$ & $3.5 \pm 0.3$ \\
\hline
\end{tabular}




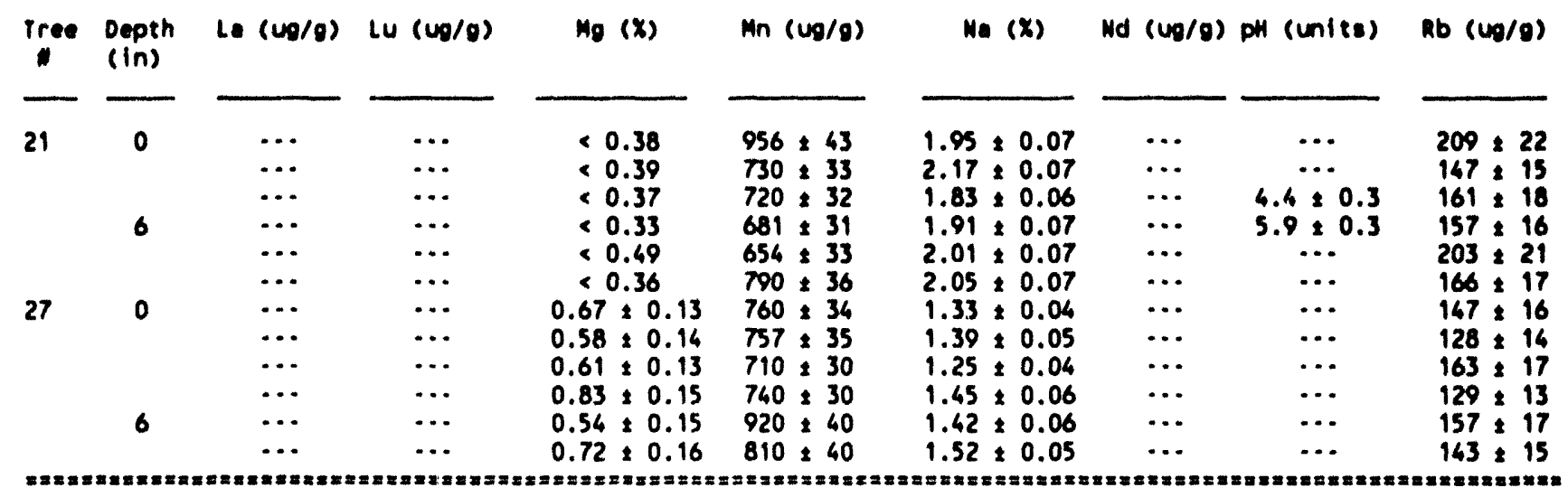

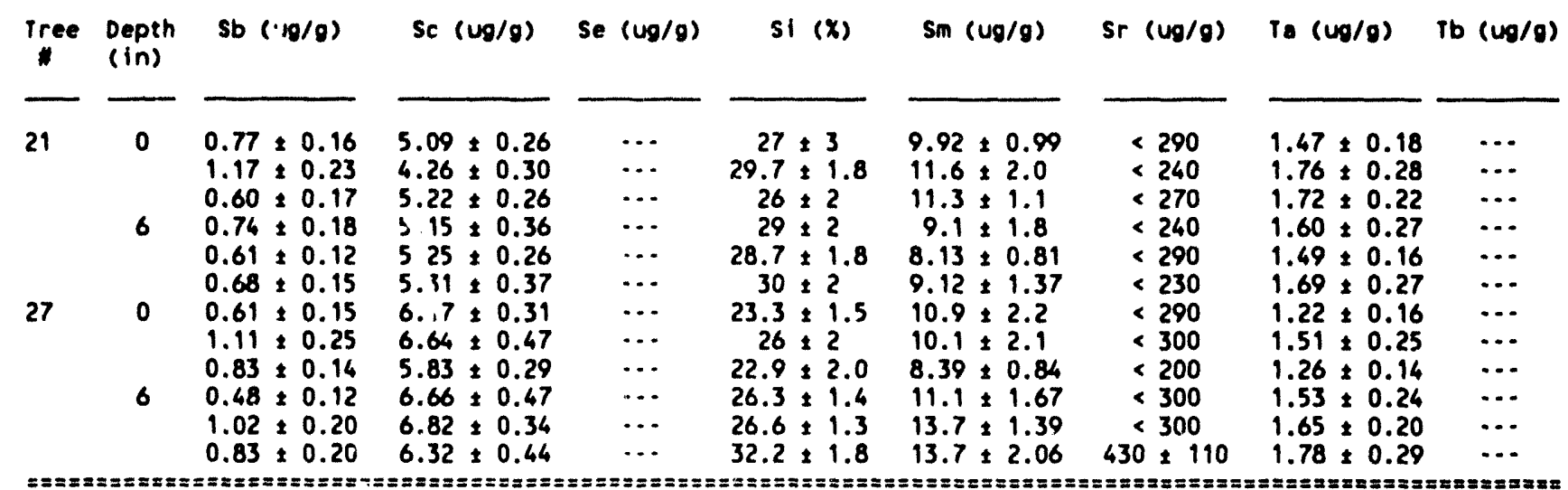

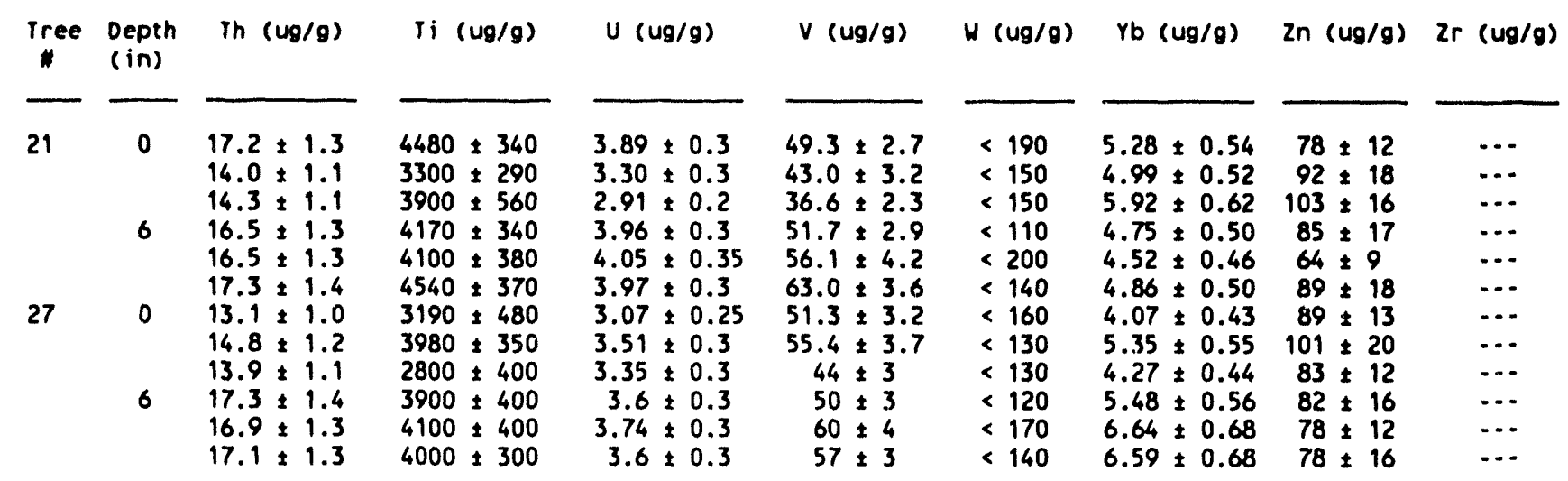


Table c.111. Individual Elemental Concentration in solls of Cactus plot 5, saguro Mational Monument

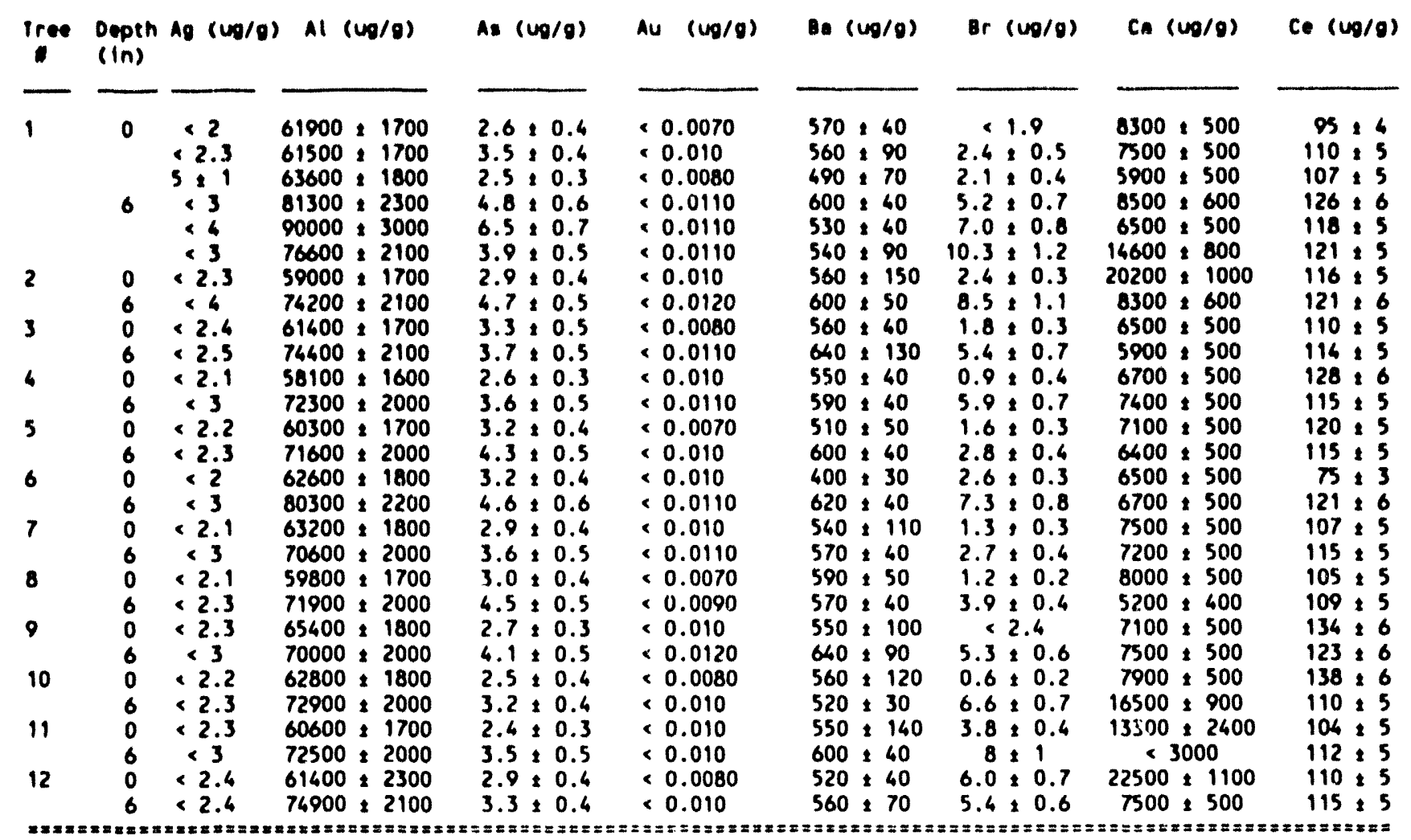

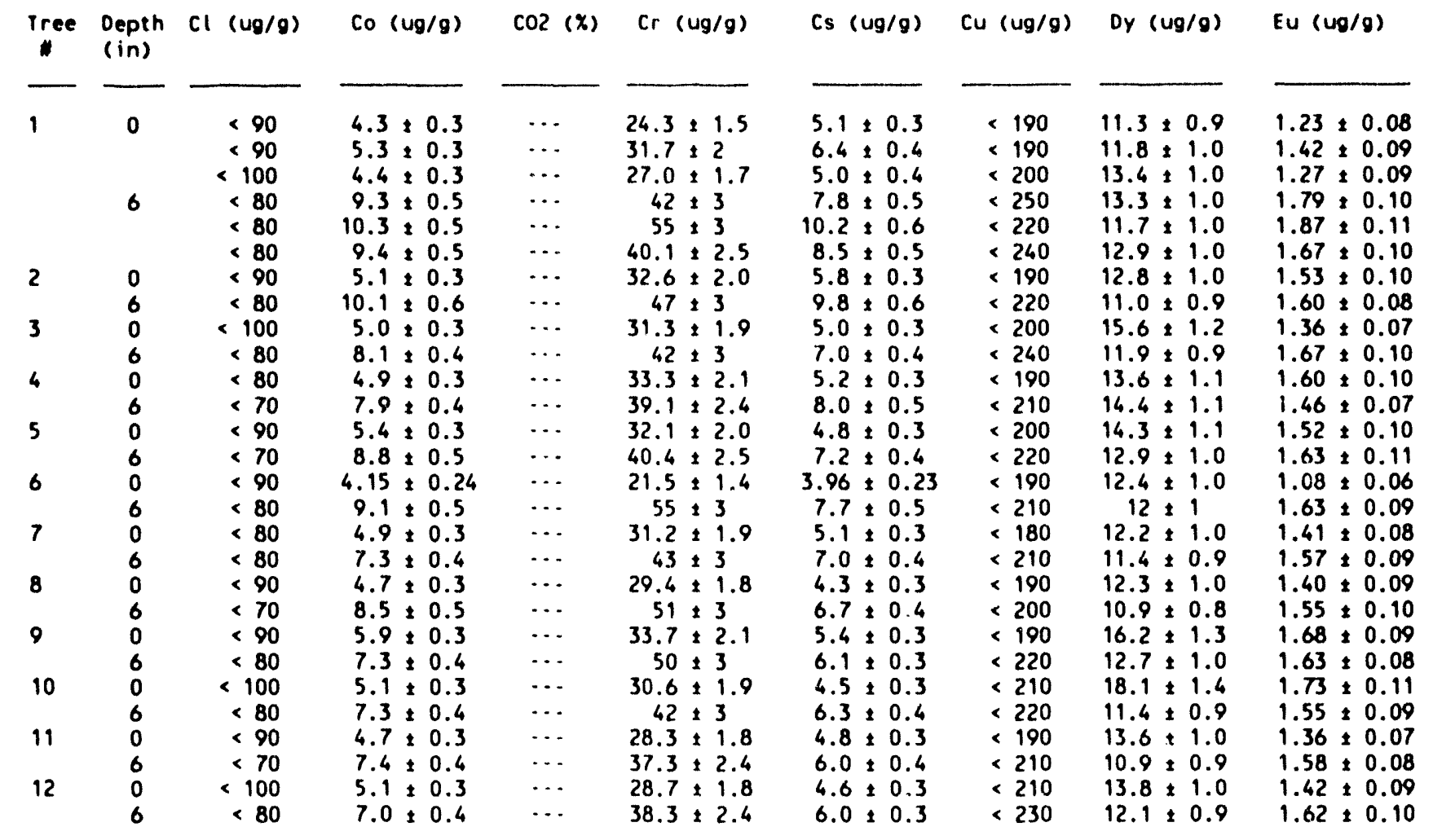




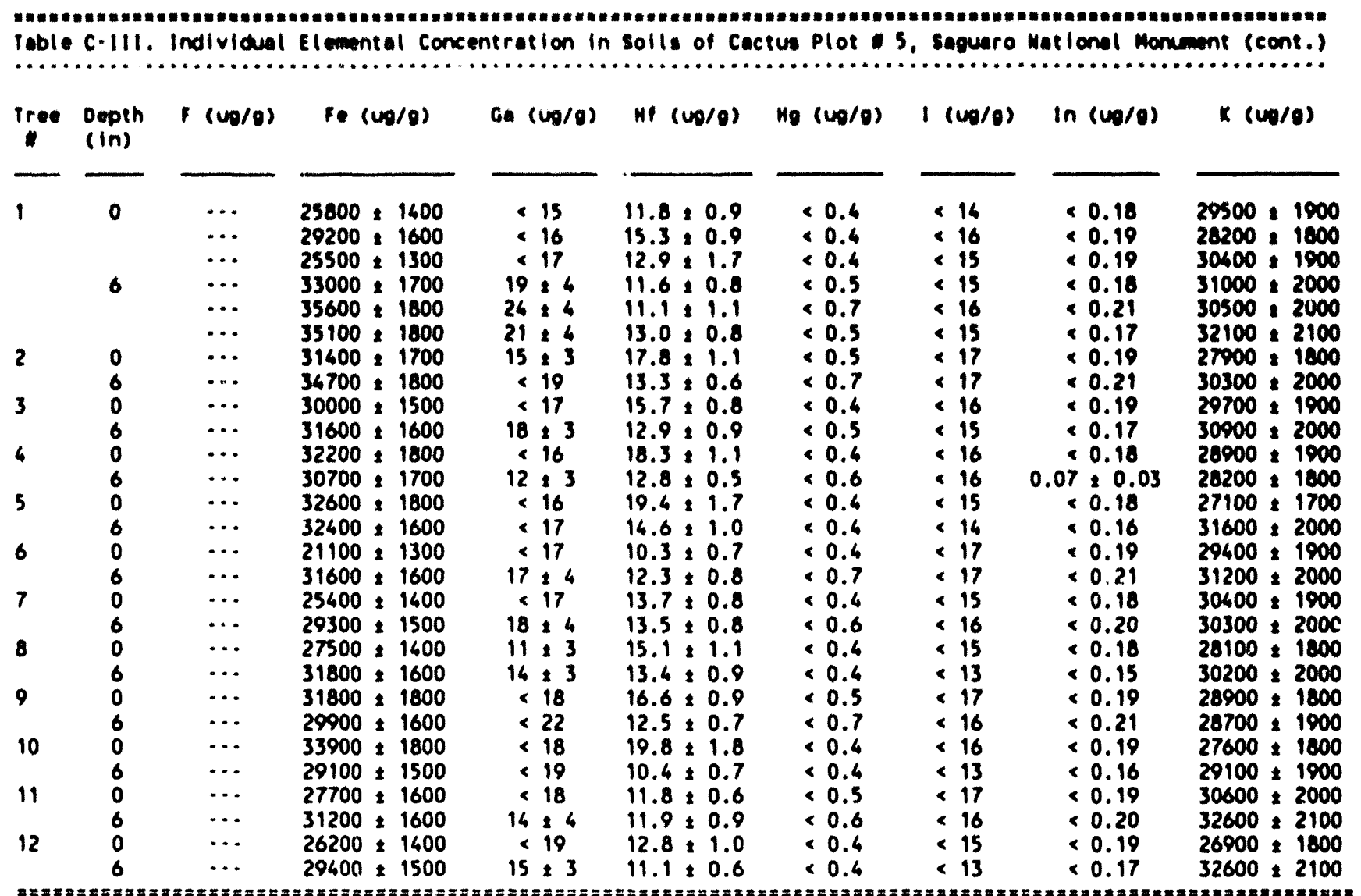

\begin{tabular}{|c|c|c|c|}
\hline Tree & $\begin{array}{l}\text { Depth } \\
\text { (in) }\end{array}$ & 1. $(u g / \theta)$ & $\operatorname{lu}(u g / g)$ \\
\hline 1 & 0 & $\begin{array}{l}48 \div 3 \\
52 \div 3 \\
52 \div 3 \\
56 \div 3 \\
54 \div 3 \\
54 \div 3\end{array}$ & $\begin{array}{l}1.35 \neq 0.06 \\
1.30 \neq 0.06 \\
1.71 \pm 0.08 \\
1.58 \neq 0.07 \\
1.30 \div 0.06 \\
1.63 \neq 0.08\end{array}$ \\
\hline 2 & $\begin{array}{l}0 \\
6\end{array}$ & $\begin{array}{l}55 \div 3 \\
55 \div 3\end{array}$ & $\begin{array}{l}1.63 \neq 0.08 \\
1.25 \neq 0.06\end{array}$ \\
\hline 3 & $\begin{array}{l}0 \\
6\end{array}$ & $\begin{array}{l}54 \pm 3 \\
53 \pm 3\end{array}$ & $\begin{array}{l}2.07 \neq 0.10 \\
1.56 \neq 0.07\end{array}$ \\
\hline 4 & $\begin{array}{l}0 \\
6\end{array}$ & $\begin{array}{l}59 \pm 3 \\
53 \pm 3\end{array}$ & $\begin{array}{l}1.68 \div 0.08 \\
1.64 \div 0.08\end{array}$ \\
\hline 5 & $\begin{array}{l}0 \\
6\end{array}$ & $\begin{array}{l}61 \pm 4 \\
52 \pm 3\end{array}$ & $\begin{array}{l}1.74 \div 0.08 \\
1.76 \div 0.08\end{array}$ \\
\hline 6 & $\begin{array}{l}0 \\
6\end{array}$ & $\begin{array}{l}52 \div 3 \\
54 \pm 3\end{array}$ & $\begin{array}{l}1.07 \neq 0.05 \\
1.43 \neq 0.07\end{array}$ \\
\hline 7 & $\begin{array}{l}0 \\
6\end{array}$ & $\begin{array}{l}52 \pm 3 \\
52 \pm 3\end{array}$ & $\begin{array}{l}1.53 \pm 0.07 \\
1.32 \pm 0.06\end{array}$ \\
\hline 8 & $\begin{array}{l}0 \\
6\end{array}$ & $\begin{array}{l}52 \div 3 \\
52 \pm 3\end{array}$ & $\begin{array}{l}1.47 \pm 0.07 \\
1.38 \div 0.06\end{array}$ \\
\hline 9 & $\begin{array}{l}0 \\
6\end{array}$ & $\begin{array}{l}62 \div 3 \\
56 \div 3\end{array}$ & $\begin{array}{l}1.99 \neq 0.09 \\
1.57 \div 0.08\end{array}$ \\
\hline 10 & $\begin{array}{l}0 \\
6\end{array}$ & $\begin{array}{l}70 \pm 4 \\
52 \pm 3\end{array}$ & $\begin{array}{l}2.29 \pm 0.11 \\
1.40 \pm 0.07\end{array}$ \\
\hline 11 & 0 & $\begin{array}{l}50 \div 3 \\
50 \div 3\end{array}$ & $\begin{array}{l}1.74 \pm 0.08 \\
1.39 \pm 0.07\end{array}$ \\
\hline 12 & $\begin{array}{l}0 \\
6\end{array}$ & $\begin{array}{l}52 \pm 3 \\
53 \pm 4\end{array}$ & $\begin{array}{l}1.78 \pm 0.08 \\
1.48 \pm 0.07\end{array}$ \\
\hline
\end{tabular}

Mg (ug/g)

Mn $(u g / g)$

$\mathrm{Ma}(u g / g)$

$3300 \pm 400$

$3600 \pm 500$

$<1400$

$4300 \div 500$

$3800 \div 500$

$3500 \pm 400$

$5100 \div 1400$

$4500 \pm 600$

$2600 \div 400$

$3400 \pm 400$

$2900 \pm 500$

$3000 \div 500$

$2600 \div 400$

$3000 \pm 400$

$3300 \div 400$

$4800 \pm 500$

$2600 \pm 400$

$3300 \div 400$

$1800+400$

$3300 \pm 400$

$3900 \pm 500$

$3400 \pm 500$

$3000 \pm 500$

$3500 \pm 400$

$3700 \pm 600$

$3000 \pm 400$

$5100 \pm 500$

$2800 \pm 400$

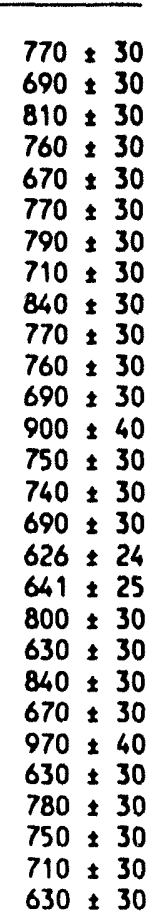

$14200 \div 600$

$14900 \div 700$

$14100 \pm 600$

$14200 \pm 600$

$12400 \div 500$

$12800 \div 600$

$13000 \div 600$

$11800 \div 500$

$13300+600$

$13600 \div 600$

$14600 \div 700$

$13100+600$

$13600 \div 600$

$13400 \div 600$

$13200 \pm 600$

$13400+600$

$14900 \div 700$

$13900 \div 600$

$13100 \div 600$

$11600 \pm 500$

$14500 \div 600$

$14300 \div 600$

$14900 \div 600$

$14900 \pm 600$

$13100 \div 600$

$13000+500$

$13300 \pm 600$

$14600 \pm 600$
Nd (ug/g) PH (units) Rb (ug/g)

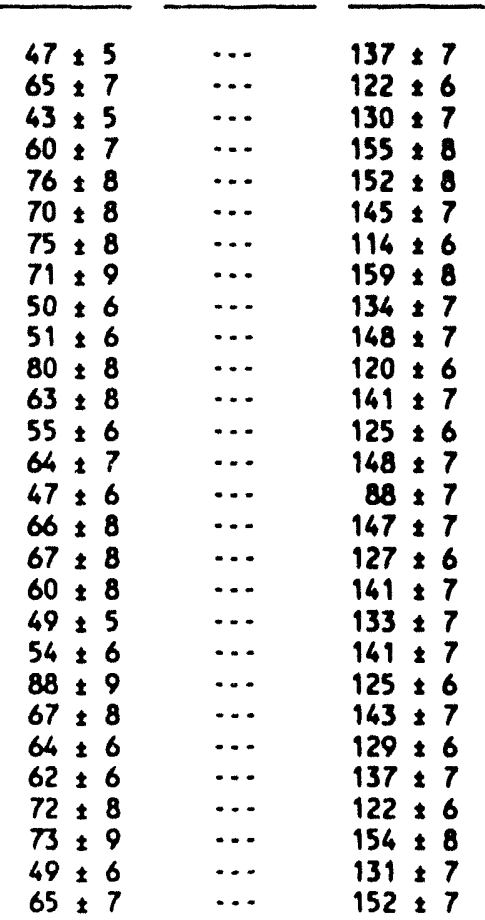




\begin{tabular}{|c|c|c|c|c|c|c|c|c|c|c|c|c|}
\hline Iree & $\begin{array}{l}\text { Depth } \\
(\ln )\end{array}$ & sb & $(\omega / \theta)$ & 86 & $(\omega 0 / 0)$ & se $(\omega s / 0)$ & $51(x)$ & $\ln (v / 9)$ & $\operatorname{se}(\omega / \theta)$ & is 1 & $(40 / \theta)$ & ib $(\omega / \theta)$ \\
\hline $\begin{array}{l}2 \\
3 \\
4 \\
5 \\
6 \\
7 \\
8\end{array}$ & $\begin{array}{l}0 \\
6 \\
0 \\
6 \\
0 \\
6 \\
0 \\
6 \\
0 \\
6 \\
0 \\
6 \\
0 \\
6 \\
0 \\
6 \\
0 \\
6 \\
0 \\
6 \\
0 \\
6\end{array}$ & $\begin{array}{l}1.06 \\
1.35 \\
1.17 \\
1.21 \\
1.30 \\
1.13 \\
1.20 \\
1.39 \\
1.16 \\
1.10 \\
1.22 \\
1.28 \\
1.13 \\
1.09 \\
1.16 \\
1.26 \\
1.32 \\
1.00 \\
1.23 \\
1.22 \\
1.31 \\
1.17 \\
1.16 \\
0.82 \\
1.06 \\
1.07 \\
0.93 \\
1.06\end{array}$ & 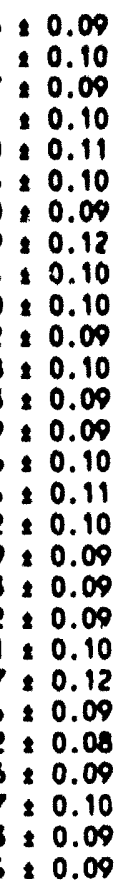 & $\begin{array}{r}6.5 \\
7.5 \\
6.3 \\
10.3 \\
11.6 \\
10.4 \\
7.3 \\
10.3 \\
7.1 \\
9.3 \\
7.0 \\
9.0 \\
7.3 \\
9.6 \\
5.4 \\
10.6 \\
6.8 \\
8.8 \\
6.8 \\
9.3 \\
8.2 \\
8.9 \\
7.8 \\
9.3 \\
7.1 \\
8.9 \\
7.2 \\
9.3\end{array}$ & 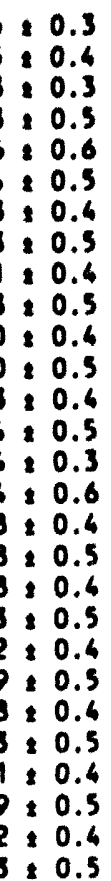 & 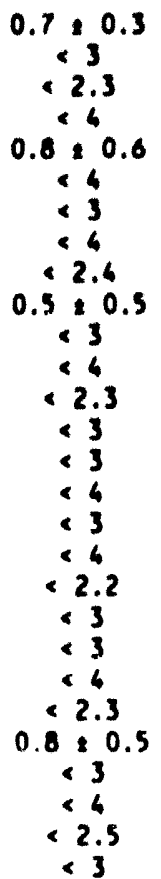 & 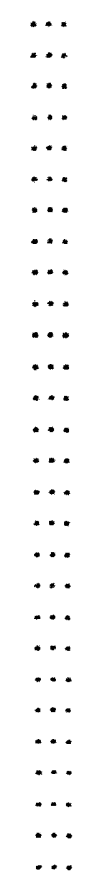 & $\begin{array}{r:r}9.6 & 0.6 \\
10.3 & 0.5 \\
10.6 & 0.5 \\
10.6 & 0.5 \\
11.4 & 0.5 \\
10.1 & 0.5 \\
10.9 & 0.5 \\
10.8 & 0.5 \\
11.0 & 0.5 \\
9.8 & 0.6 \\
11.6 & 0.5 \\
10.6 & 0.5 \\
11.9 & 0.5 \\
9.4 & 0.6 \\
9.7 & 0.6 \\
10.6 & 0.5 \\
10.1 & 0.5 \\
10.5 & 0.5 \\
10.2 & 0.5 \\
9.3 & 0.6 \\
12.5 & 0.6 \\
11.7 & 0.5 \\
16.2 & 0.6 \\
9.9 & 0.6 \\
9.6 & 0.6 \\
10.2 & 0.5 \\
10.6 & 0.5 \\
9.9 & 0.4\end{array}$ & 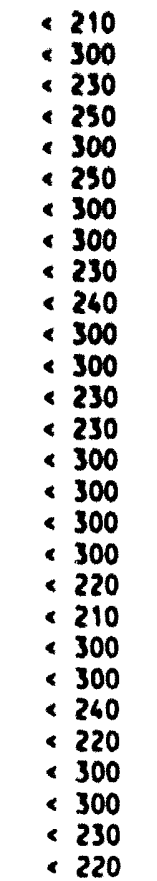 & $\begin{array}{l}0.97 \\
1.27 \\
1.07 \\
1.12 \\
1.27 \\
1.31 \\
1.16 \\
1.30 \\
1.36 \\
1.17 \\
1.36 \\
1.23 \\
1.64 \\
1.26 \\
0.84 \\
1.20 \\
1.08 \\
1.25 \\
1.13 \\
1.18 \\
1.26 \\
1.10 \\
1.38 \\
1.07 \\
1.20 \\
1.39 \\
1.21 \\
1.08\end{array}$ & $\begin{array}{l}0.07 \\
0.09 \\
0.10 \\
0.08 \\
0.00 \\
0.09 \\
0.11 \\
0.09 \\
0.10 \\
0.08 \\
0.10 \\
0.08 \\
0.10 \\
0.08 \\
0.07 \\
0.08 \\
0.08 \\
0.08 \\
0.08 \\
0.08 \\
0.08 \\
0.08 \\
0.00 \\
0\end{array}$ & $\begin{array}{l}1.42: 0.13 \\
1.69: 0.16 \\
1.70: 0.17 \\
1.99: 0.19 \\
1.77: 0.17 \\
1.92: 0.17 \\
1.69: 0.16 \\
1.70: 0.17 \\
2.10: 0.19 \\
1.81: 0.17 \\
1.85: 0.16 \\
1.92: 0.18 \\
1.82: 0.17 \\
1.89: 0.18 \\
1.19: 0.11 \\
1.78: 0.17 \\
1.65: 0.15 \\
1.73: 0.16 \\
1.68: 0.16 \\
1.70: 0.16 \\
2.19: 0.20 \\
1.91: 0.18 \\
2.23: 0.21 \\
1.80: 0.17 \\
1.75: 0.16 \\
1.64: 0.15 \\
1.79: 0.17 \\
1.82: 0.17\end{array}$ \\
\hline
\end{tabular}

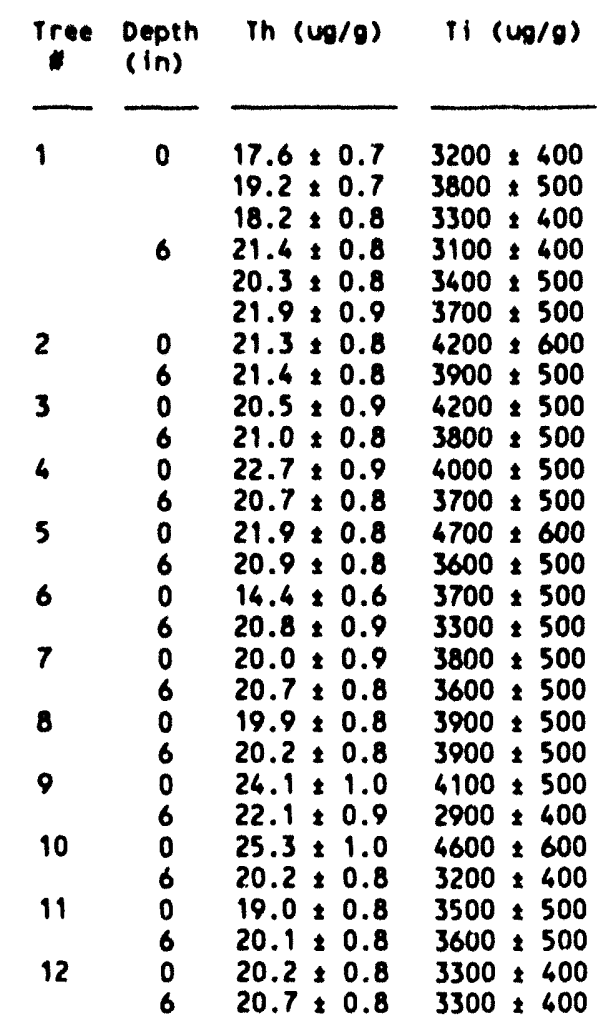

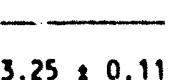

$3.68 \div 0.1348 .5 \div 2.5$

$3.54 \div 0.12$

$3.47 \div 0.13$

$3.26 \div 0.12$

$3.31 \div 0.12$

$3.98 \div 0.14$

$3.41 \div 0.12$

$4.64 \div 0.16$

$3.66 \div 0.13$

$4.06=0.14$

$3.93 \div 0.14$

$4.37 \div 0.15$

$3.76 \div 0.13$

$3.51 \div 0.12$

$3.49 \div 0.13$

$3.66 \div 0.13$

$3.41 \div 0.12$

$3.73 \div 0.13$

$3.66 \div 0.13$

$4.42 \div 0.15$

$3.45 \div 0.12$

$4.43 \div 0.16$

$3.05 \div 0.11$

$3.38 \div 0.12$

$3.82 \div 0.13$

$3.55 \div 0.12$

$3.03 \div 0.11$

\section{$47 \div 3$}

$55 \div 3$

$68: 3$

$54 \div 3$

$54 \div 3$

$63 \div 3$

$51 \div 3$

$57: 3$

$53 \div 3$

$59 \div 3$

$63 \div 3$

$56 \div 3$

$51 \div 3$

$53: 3$

$53 \div 3$

$57 \div 3$

$60 \div 3$

$52 \div 3$

$56 \div 3$

$52+3$

$46 \pm 3$

$47 \div 3$

$54 \pm 3$

$49+3$

$51 \div 3$
$V(u g / g) \quad W(u g / g)$

$44.6 \div 2.3$
$1.9: 0.5$

$3.3: 0.5$

$2.0 \div 0.4$

$2.3: 0.9$

$3.0: 0.7$

$2.1 \div 0.5$

$2.2 \div 0.6$

$1.9 \div 0.7$

$3.8 \div 0.6$

$2.9 \div 0.5$

$2.0 \div 0.4$

$2.8: 0.5$

$2.6 \div 0.6$

$2.7 \div 0.6$

$2.9: 0.7$

$3.1,0.6$

$1.4 \div 0.4$

$3.0 \div 0.5$

$3.1 \div 0.6$

$2.5+1.9$

$1.7 \div 0.6$

$2.4 \div 0.5$

$3.3 \div 0.7$

$2.8 \div 0.6$

$2.5 \div 1.1$

$2.5 \div 0.6$

$2.2 \div 0.5$

$2.7: 0.7$
Yo $(u g / g)$

In $(\omega 0 / 0)$

$\operatorname{2r}(\omega 0 / 9)$

$10.0 \div 0.5$

$9.6: 0.5$

$12.5: 0.6$

$11.3 \div 0.6$

$9.0 \div 0.5$

$11.0: 0.6$

$11.5 \div 0.6$

$8.9 \div 0.5$

$16.6 \div 0.7$

$10.3 \div 0.5$

$11.9: 0.6$

$12.0 \div 0.6$

$13.0 \div 0.7$

$12.0 \div 0.6$

$11.0: 0.6$

$10.7 \div 0.6$

$10.1: 0.5$

$9.3 \div 0.5$

$10.9 * 0.6$

$9.5 \div 0.5$

$14.8 \div 0.7$

$11.0 \div 0.6$

$16.3 \div 0.8$

$10.1: 0.5$

$12.3 \div 0.6$

$9.8 \div 0.6$

$12.5 \div 0.6$

$9.8 \div 0.5$
$43 \div 15 \quad 370 \div 50$

$61 \div 670 \div 60$

$27: 17 \quad 430: 80$

$70 \div 9720 \div 110$

$73 \div 10 \quad 560: 90$

$73: 10 \quad 910: 160$

$65: 9 \quad 530 \div 120$

$68: 9 \quad 890: 120$

$28 \div 18590 \div 70$

$64 \div 9 \quad 780 \div 110$

$50: 7 \quad 560: 100$

$55 \div 8710 \div 100$

$50 \div 16 \quad 620 \div 70$

$63 \div 840 \div 140$

$46 \div 6 \quad 320 \div 50$

$65: 9 \quad 730 \div 130$

$52 \div 7 \quad 440 \div 70$

$48 \div 7780 \div 210$

$36 \div 15 \quad 480 \div 60$

$63: 820 \div 130$

$65 \div 9510 \div 80$

$58: 8 \quad 740 \div 100$

$41 \div 15 \quad 660 \div 80$

$62 \div 8 \quad 610 \div 100$

$48 \div 7330 \div 60$

$54 \div 7 \quad 700 \div 130$

$38 \div 19 \quad 420 \div 60$

$64 \div 9680 \div 100$ 


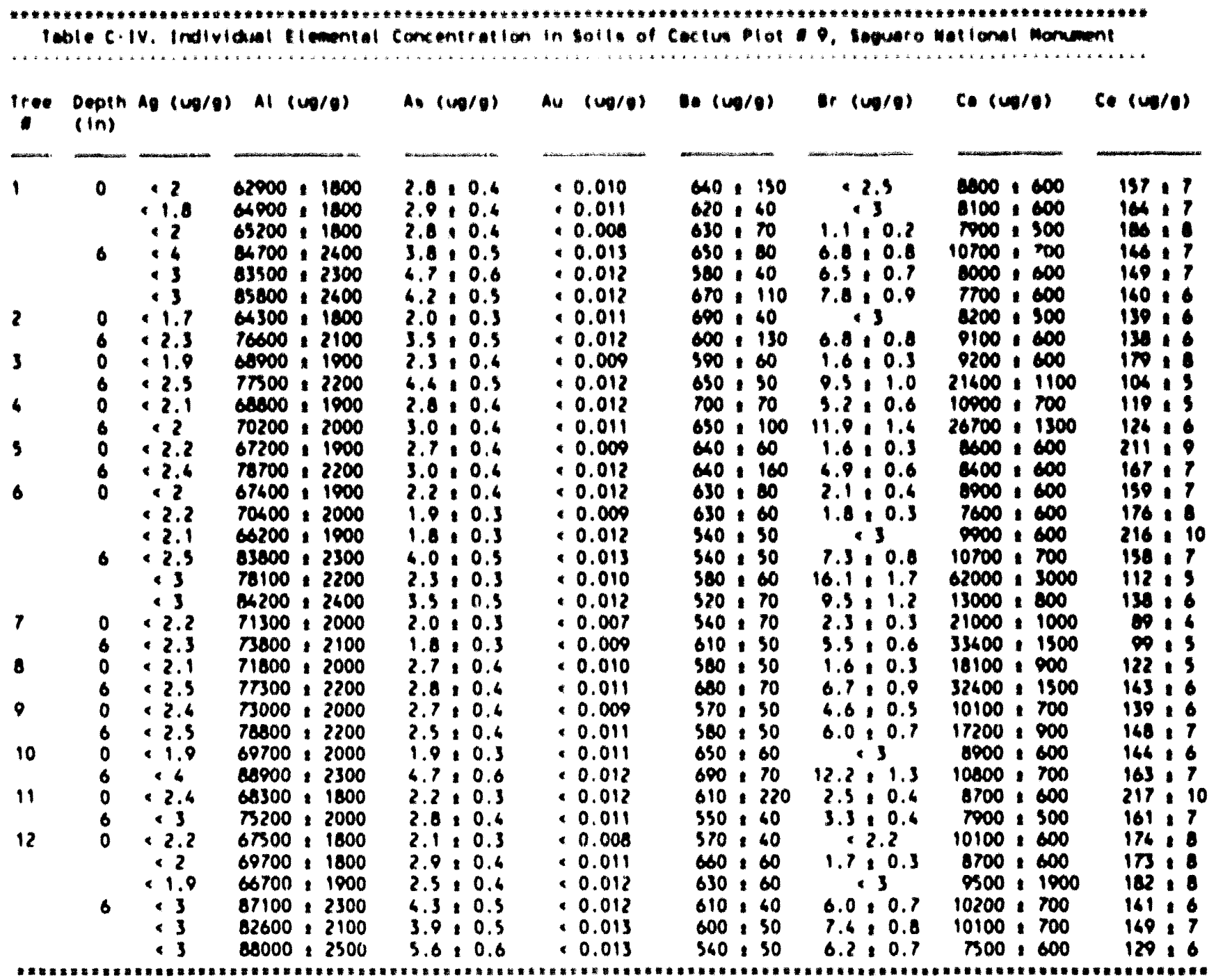

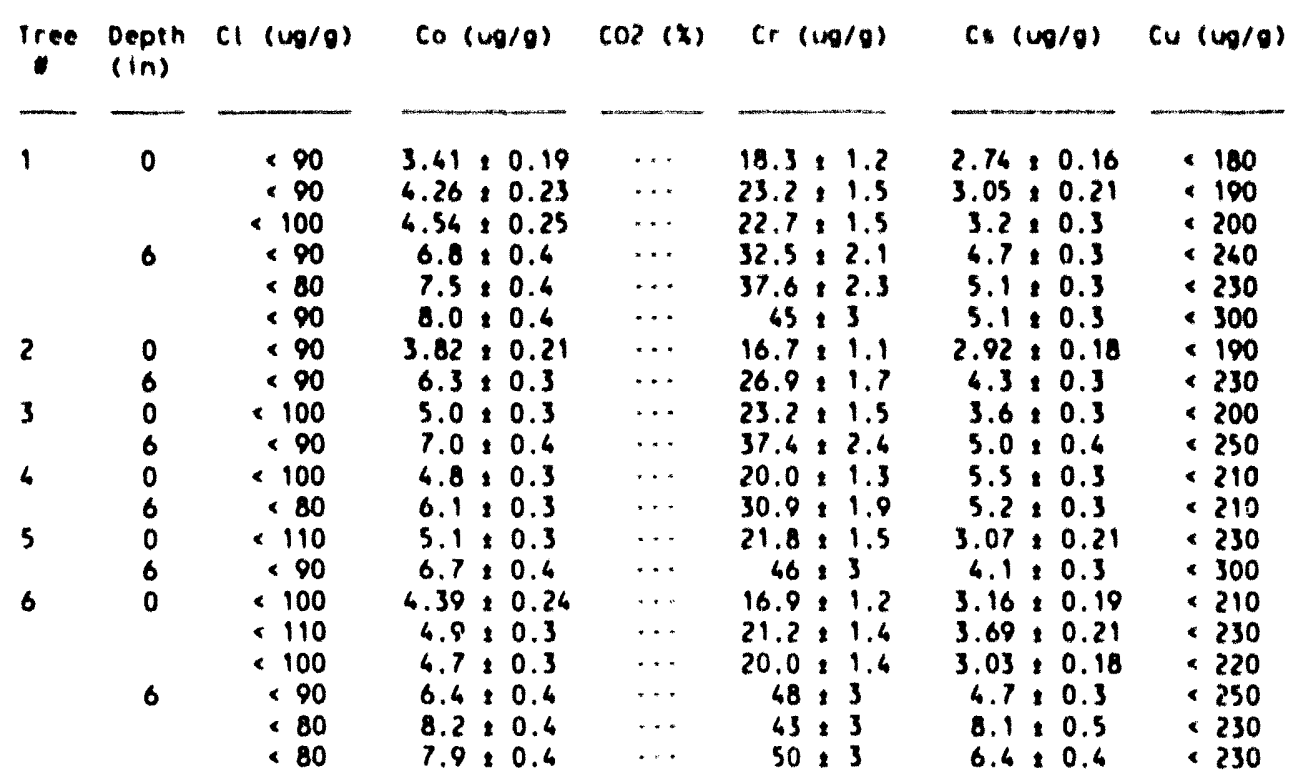

Or $(w 0 / g)$

Iu $(00 / 0)$

$16.0 \div 1.3$

$15.6,1.2$

$18.5 \div 1.5$

$13.6: 1.1$

$13.7: 1.6$

$13.7,1.1$

$13.6: 1.1$

$13.7: 1.2$

$17.4: 1.3$

$8.0: 0.7$

$10.6: 0.9$

$11.2: 0.9$

$20.0 \div 1.5$

$16.5: 1.2$

$16.6: 1.3$

$18.2: 1.6$

$21.6,1.8$

$15.2,1.2$

$12.2=0.9$

$12.8 \div 1.0$
$1.06=0.09$

$2.11: 0.11$

$2.36: 0.16$

$2.22: 0.12$

$1.97: 0.08$

$1.07: 0.09$

$1.80: 0.10$

$1.90: 0.13$

$2.30: 0.12$

$1.67: 0.07$

$1.70 \div 0.08$

$1.87: 0.10$

$2.57: 0.13$

$2.22: 0.12$

$2.00: 0.10$

$2.14: 0.10$

$2.70: 0.13$

$2.09: 0.13$

$1.7 \times 0.10$

$1.02 \cdot 0.09$ 
(4)

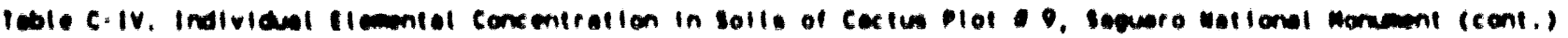

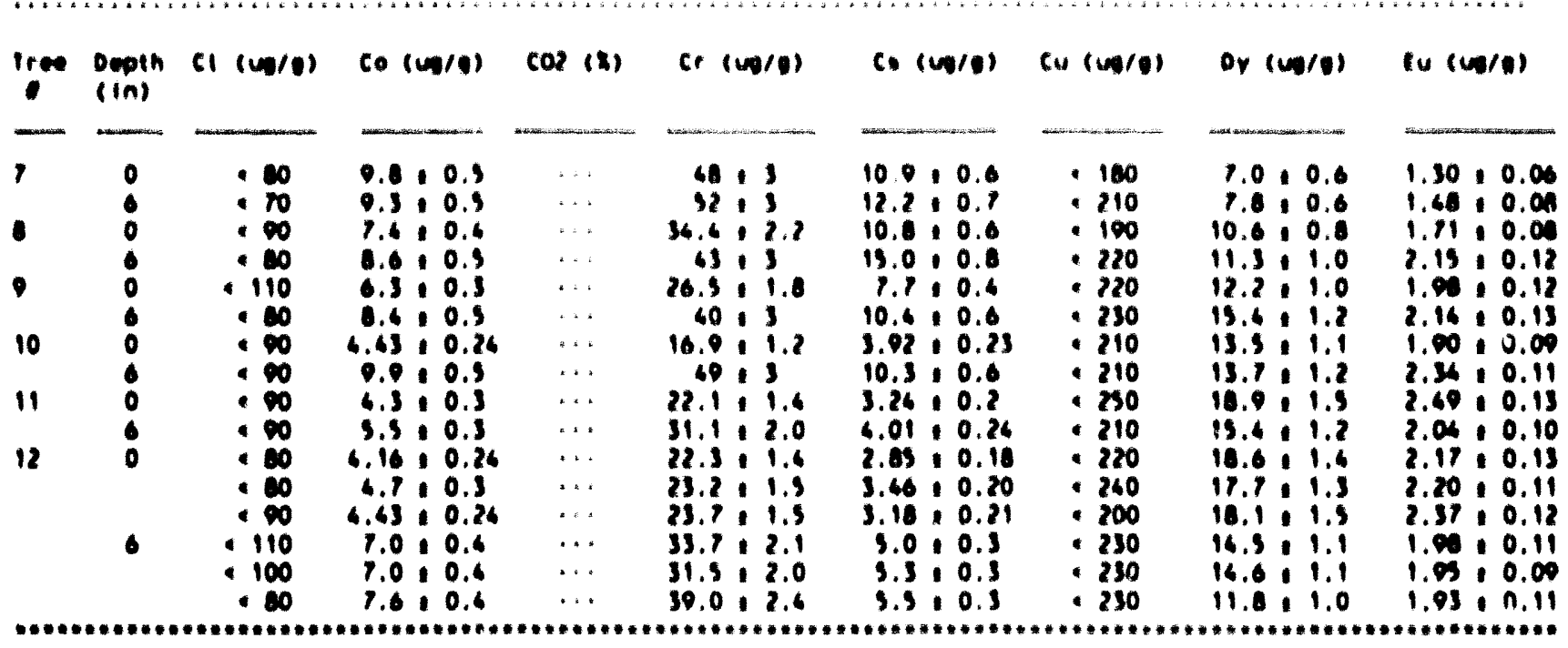

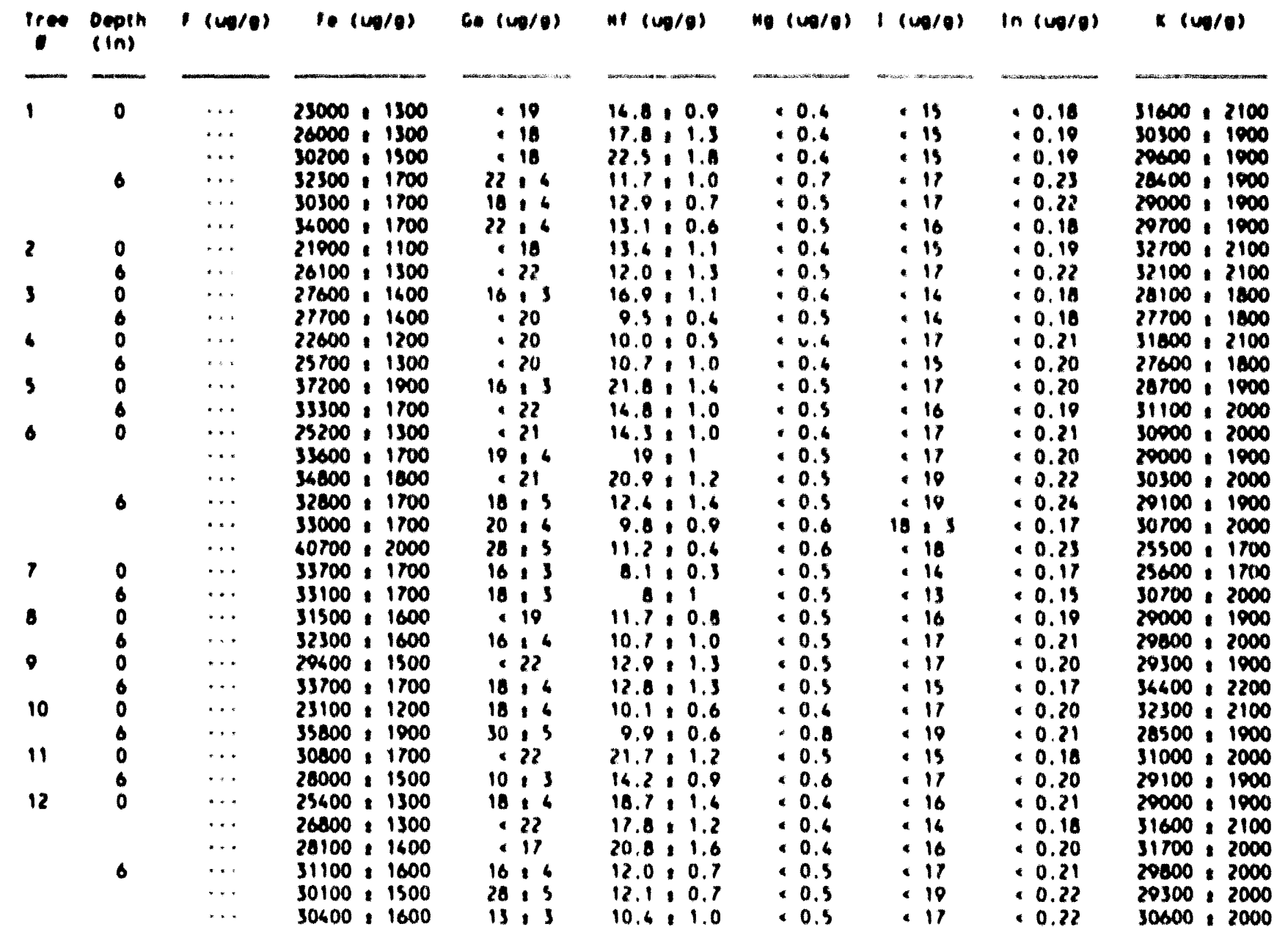




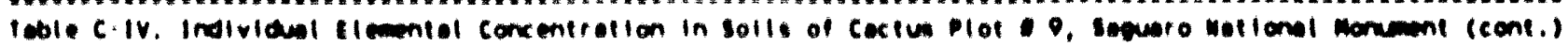

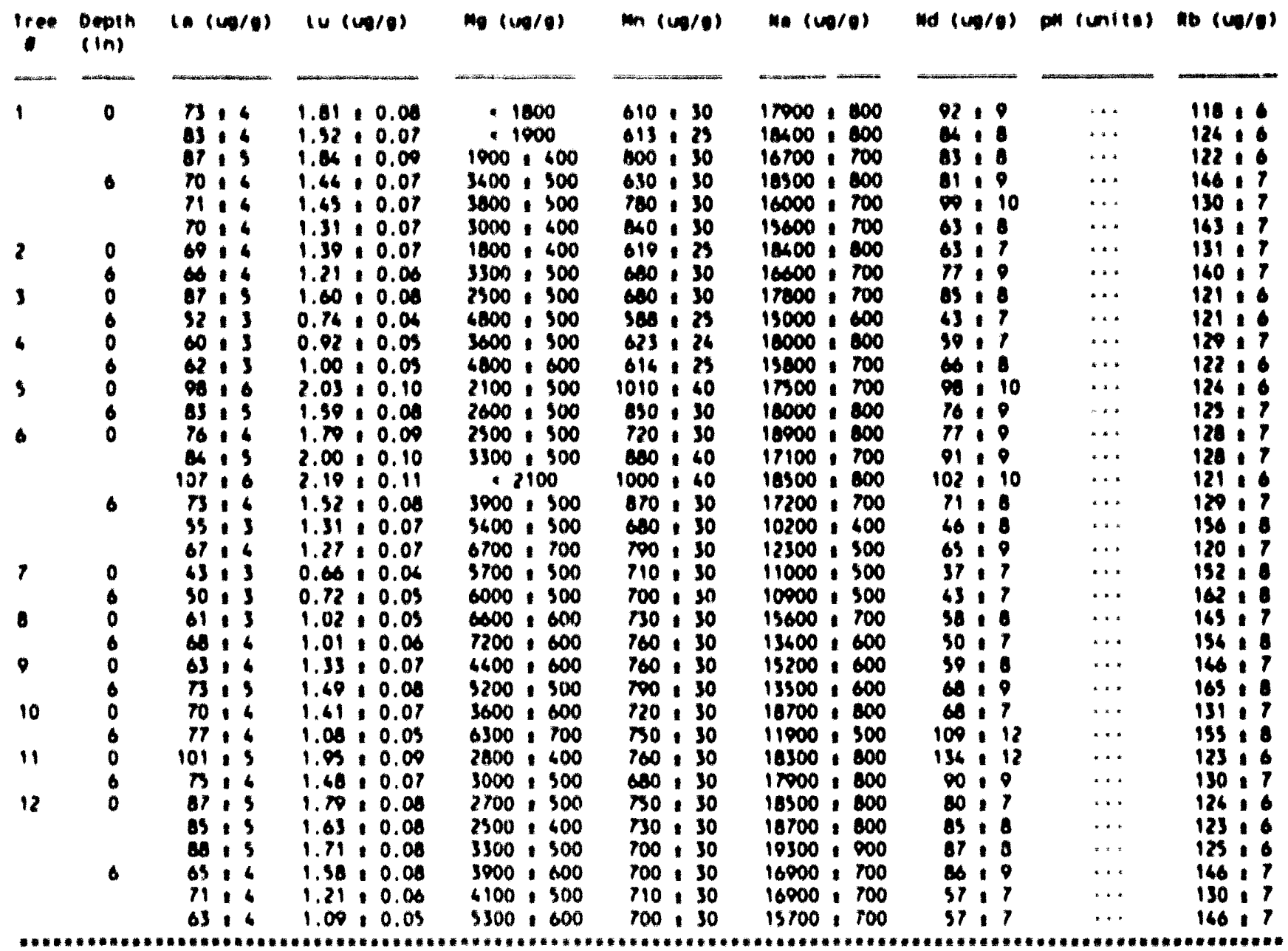

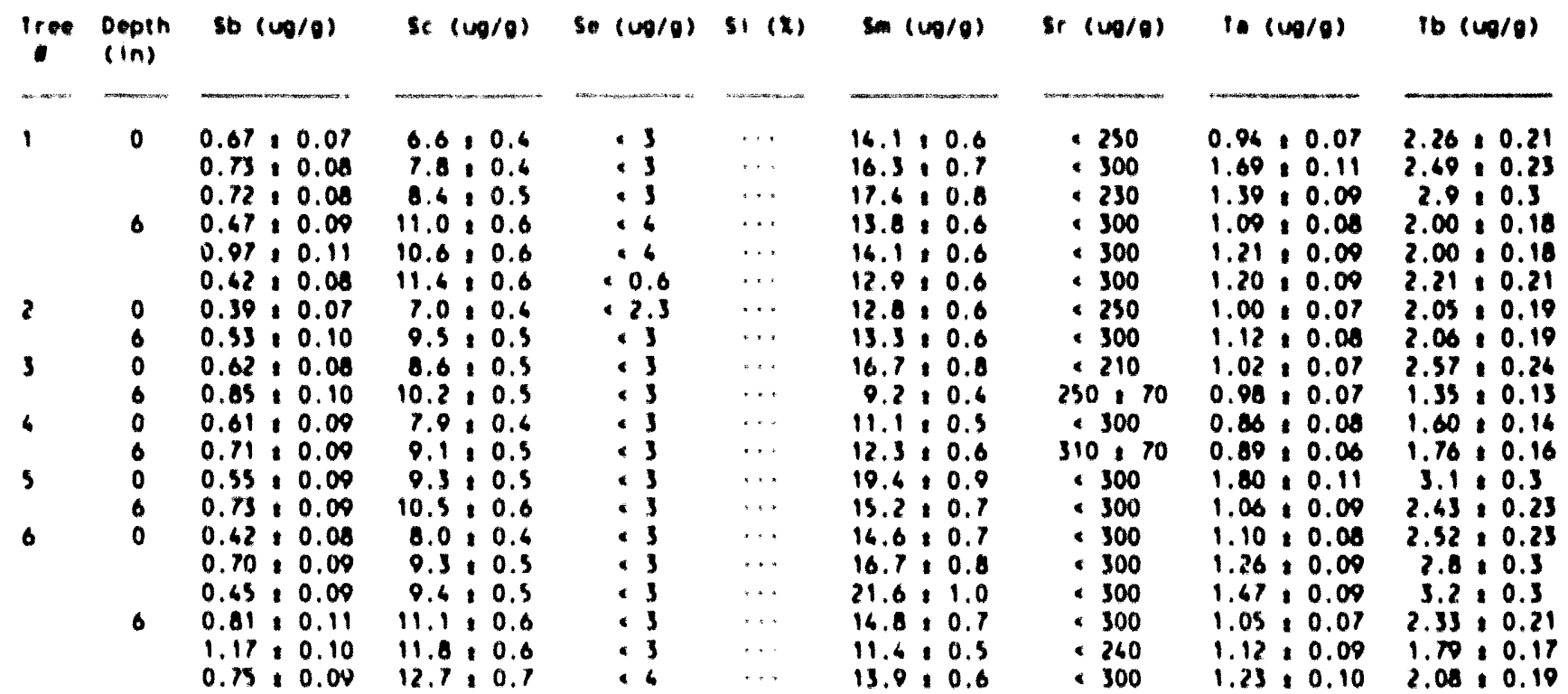




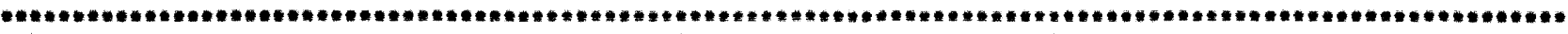

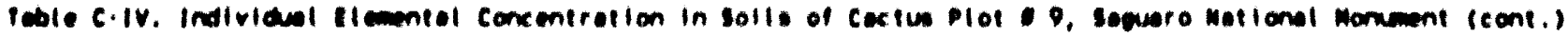

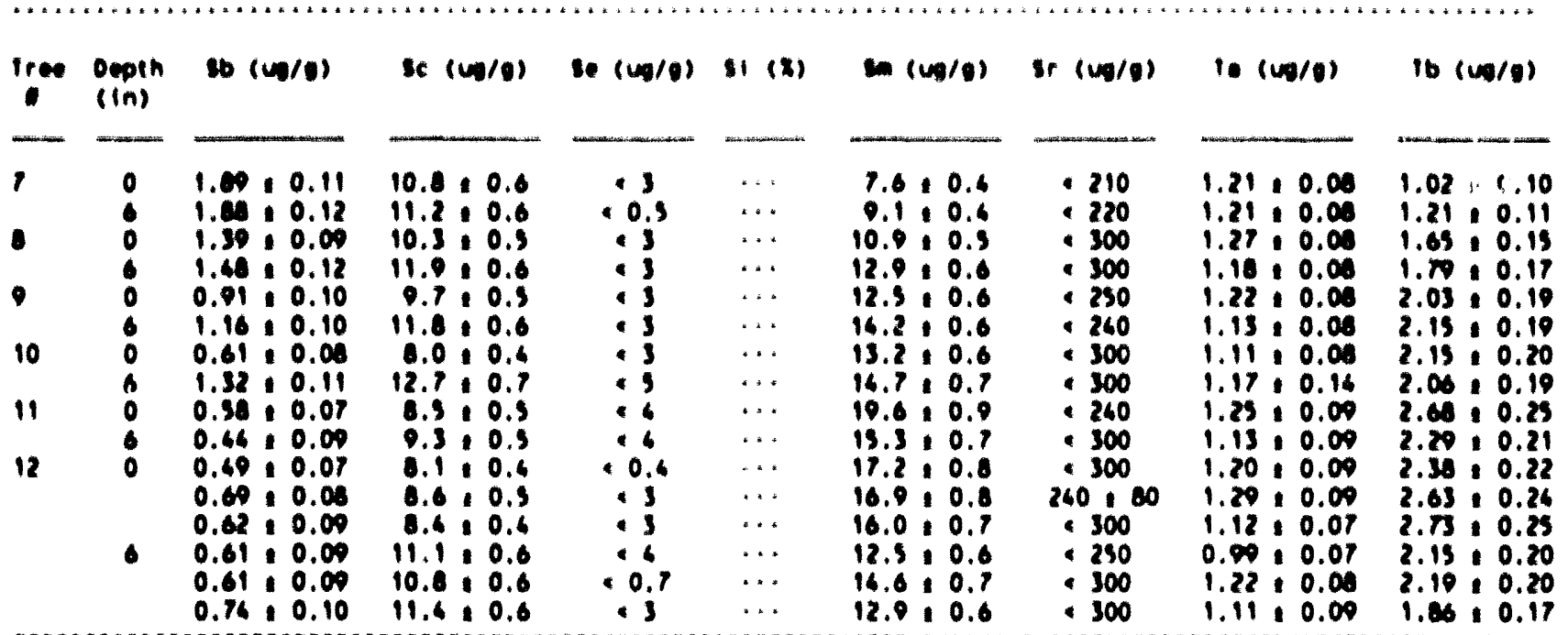

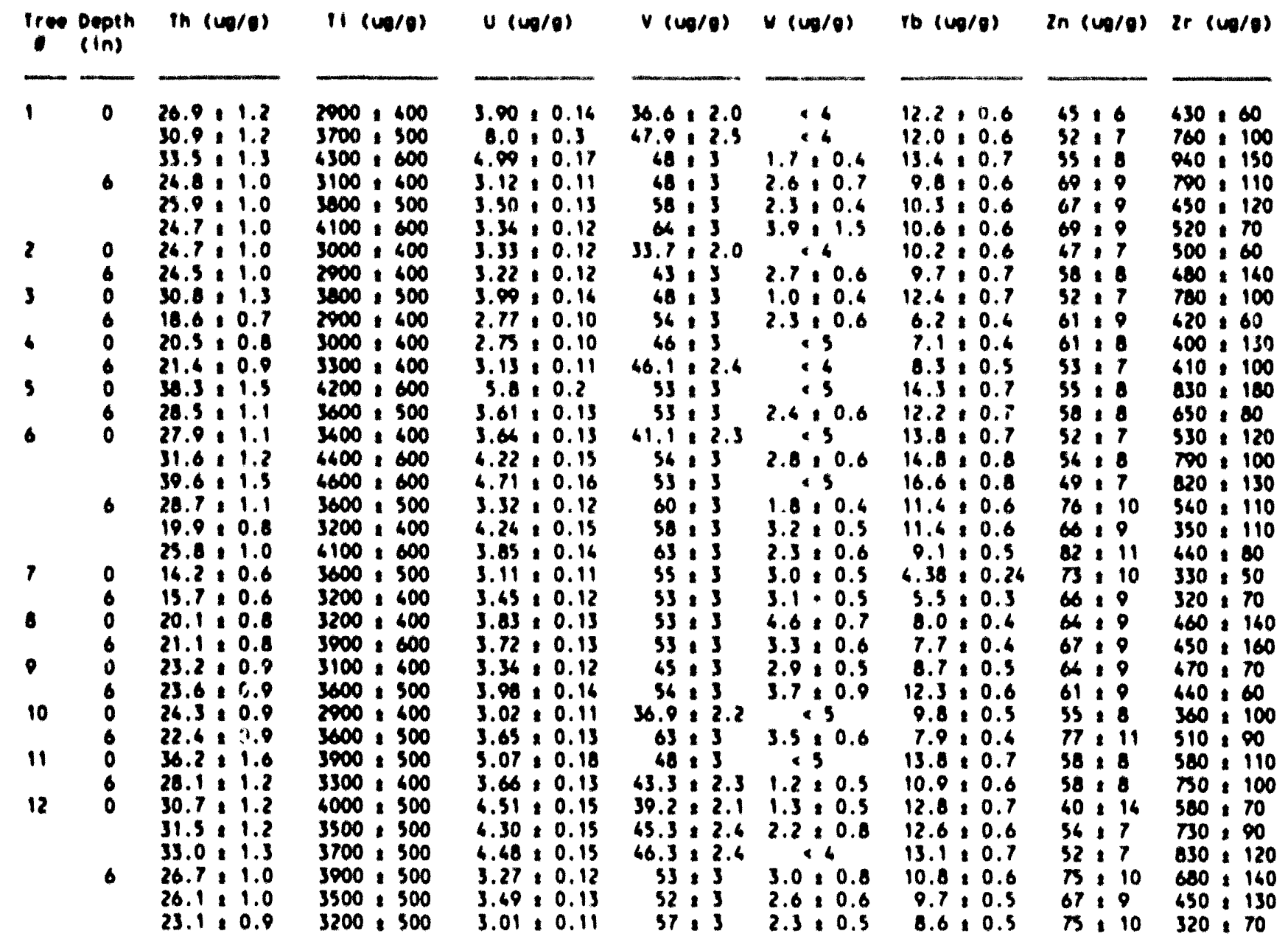




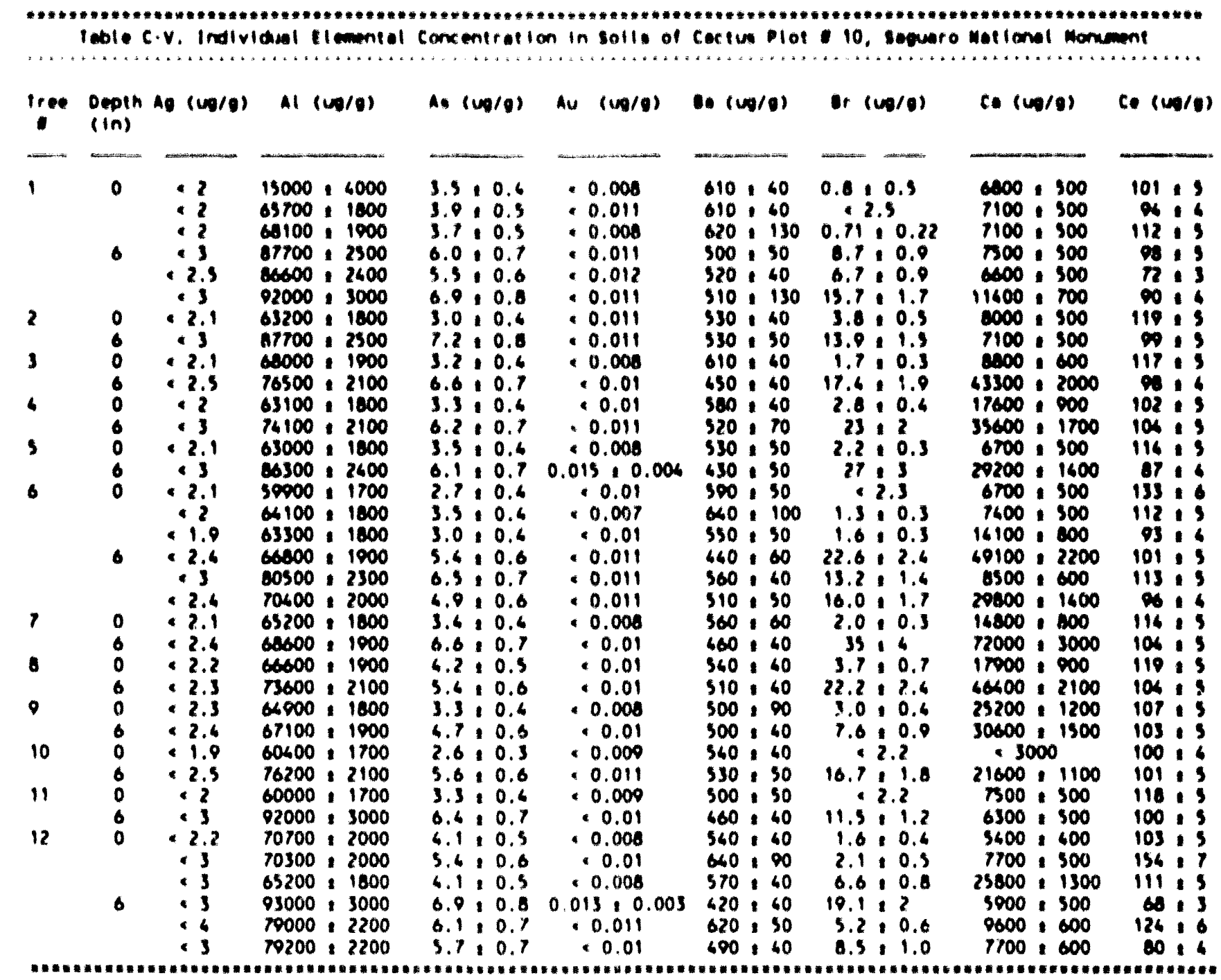

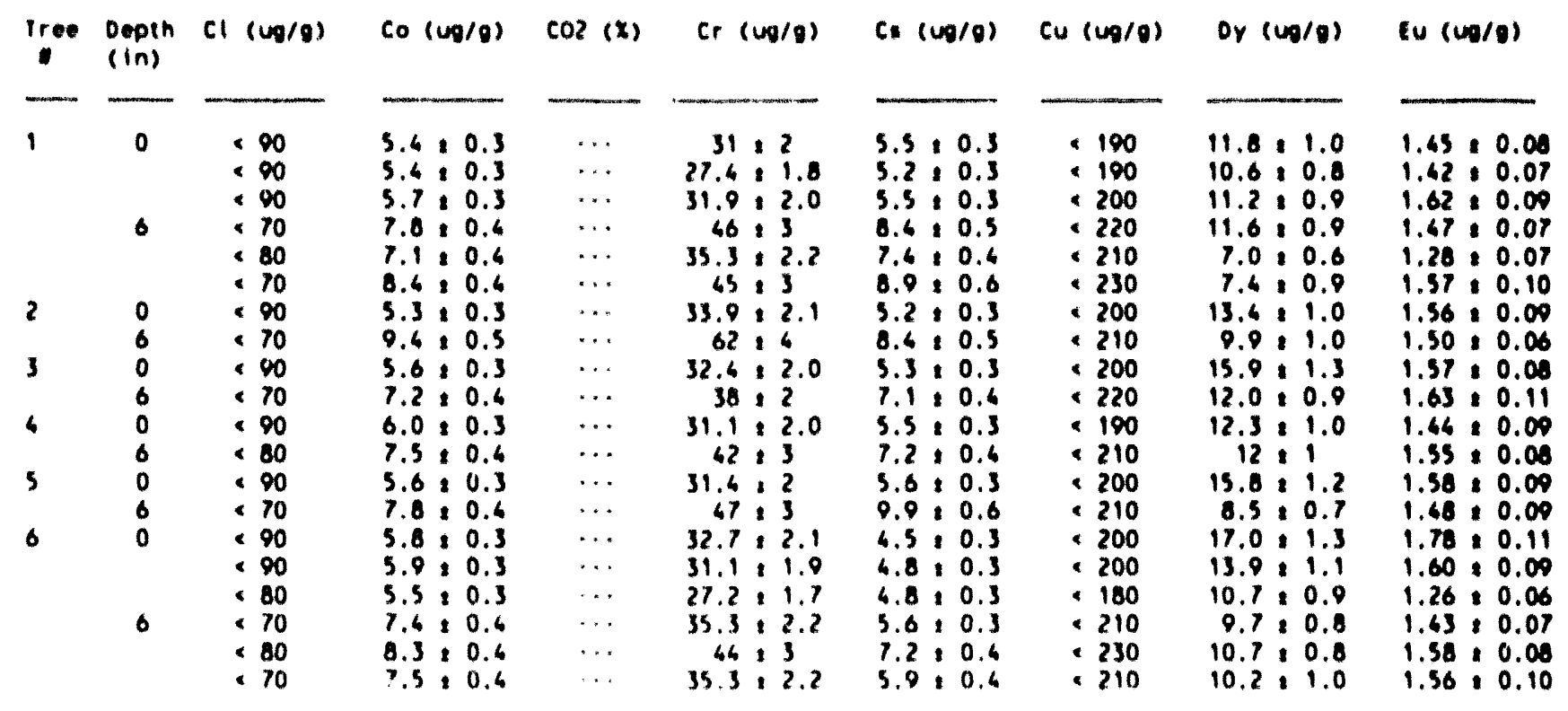




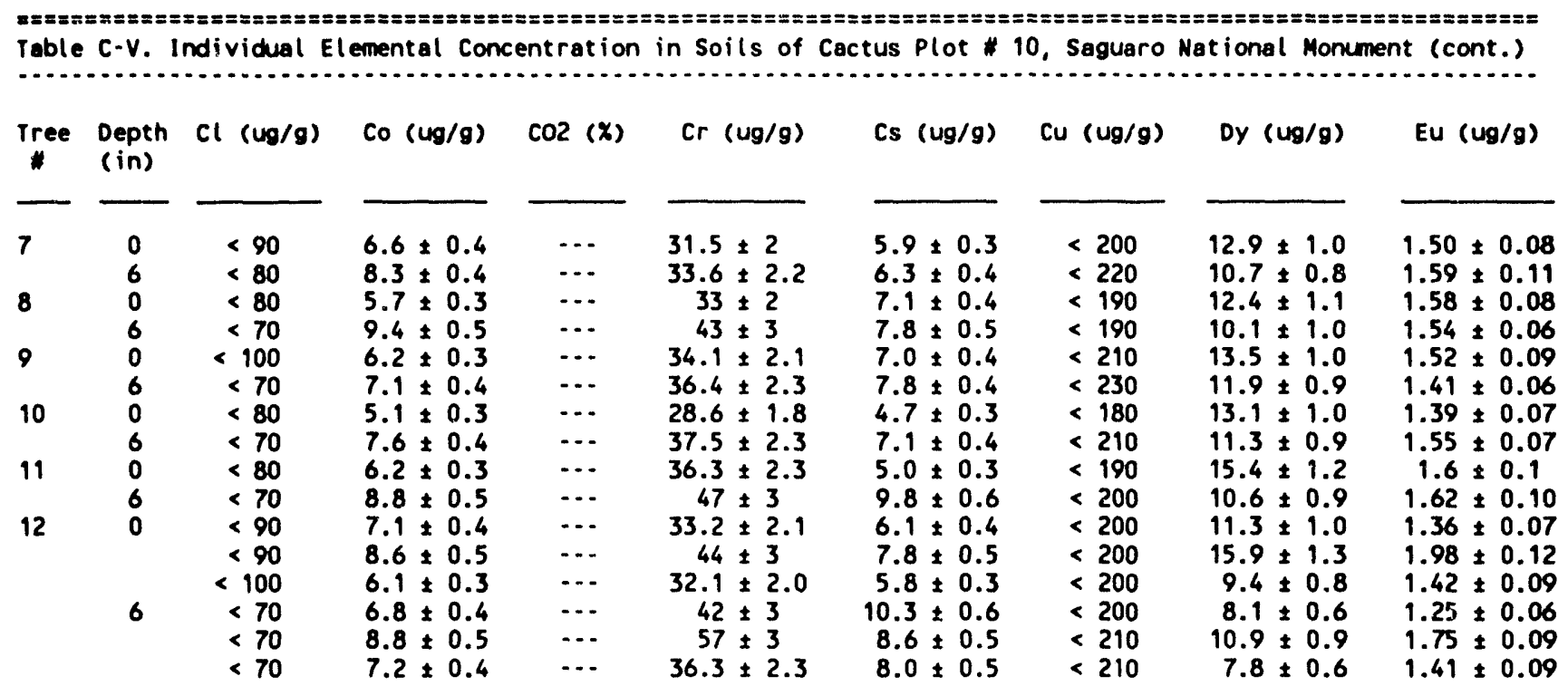

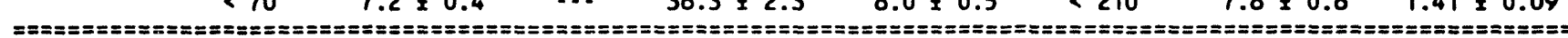

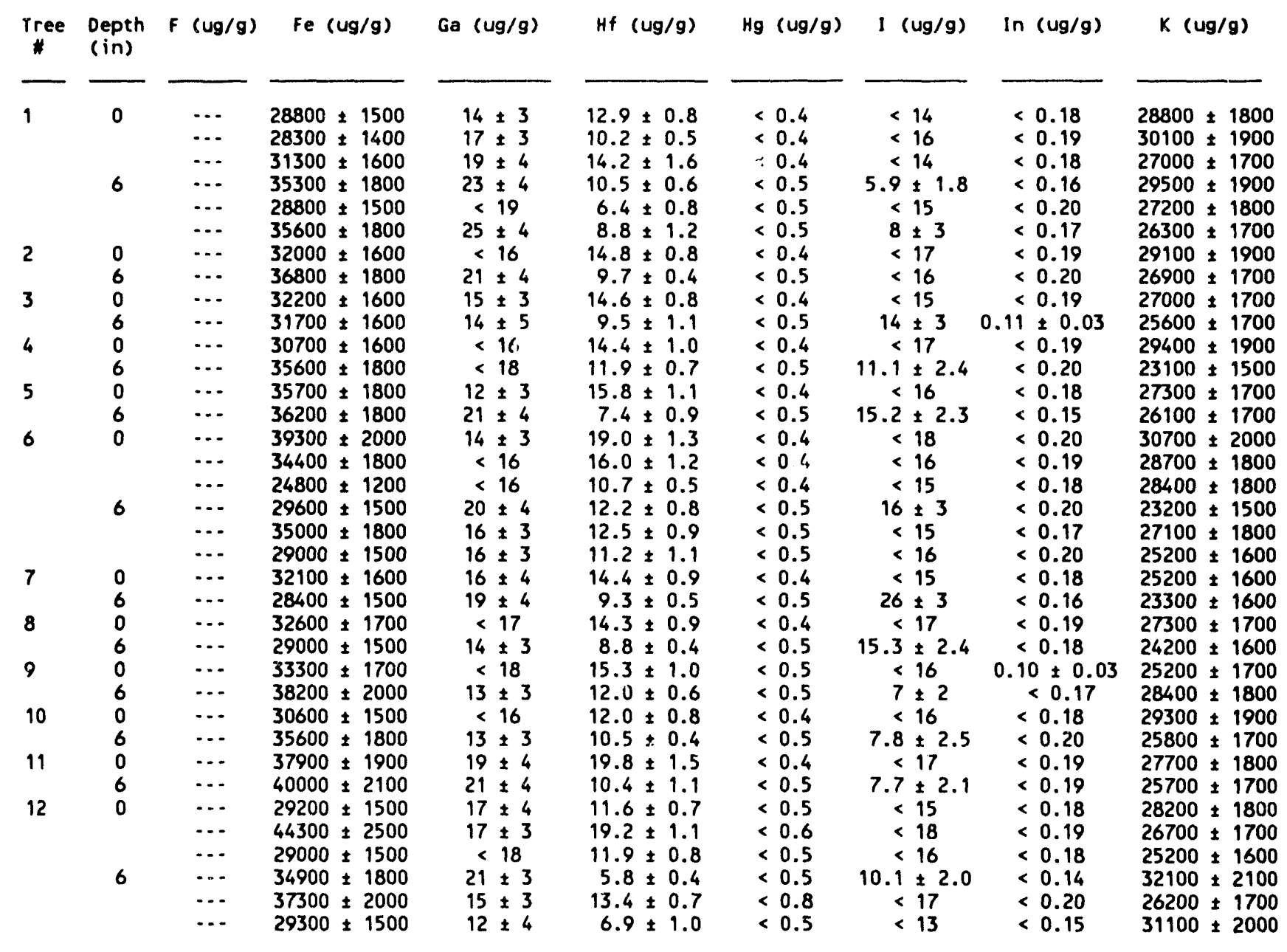




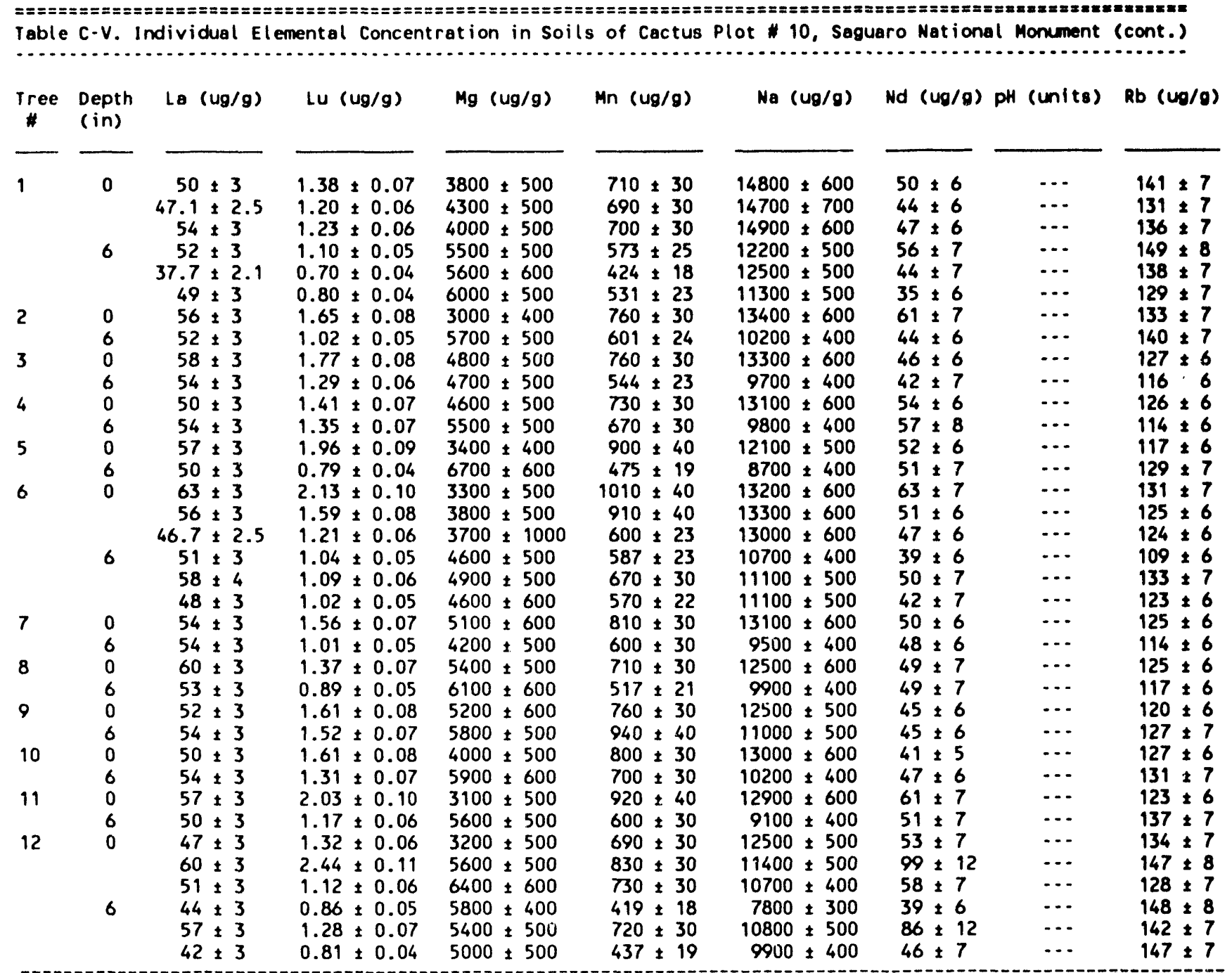

$============-==-42 \pm 3 \quad 0.81 \pm 0.04 \quad 5000 \pm 500$

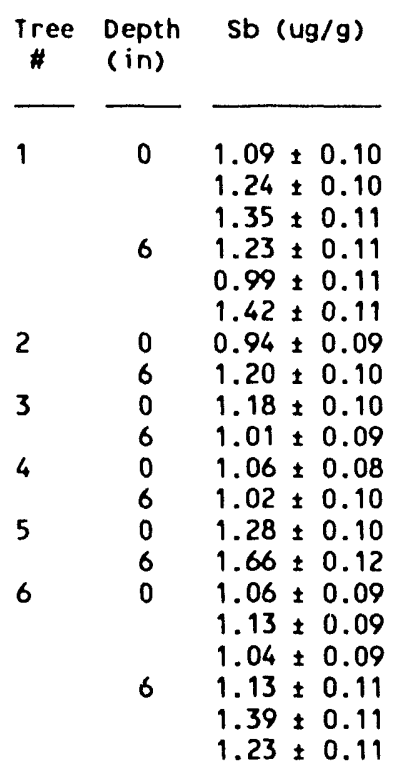

Se $(u g / g)$ si (\%)

$\operatorname{Sm}(u g / g) \quad \operatorname{Sr}(u g / g)$

Ta $(u g / g)$

Tb $(u g / g)$

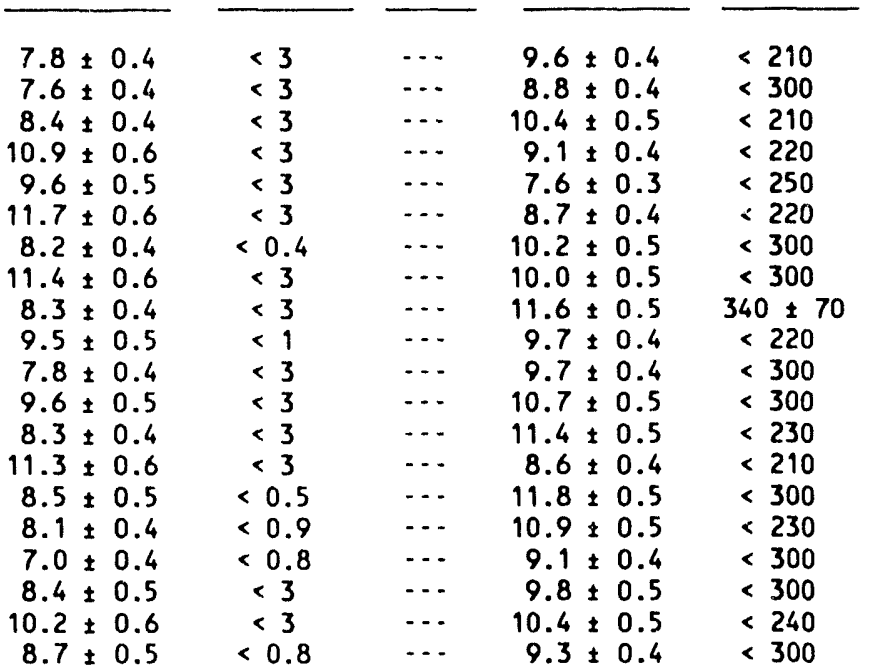

$1.16 \pm 0.08$

$1.02 \pm 0.08$

$1.20 \pm 0.12$

$0.97 \pm 0.07$

$0.79 \pm 0.06$

$1.00 \pm 0.09$

$1.32 \pm 0.08$

$1.10 \div 0.08$

$1.16 \pm 0.08$

$1.14 \pm 0.08$

$1.09 \pm 0.09$

$1.17 \pm 0.08$

$1.53 \pm 0.10$

$1.09+0.08$

$1.52 \pm 0.10$

$1.32 \pm 0.09$

$0.93 \pm 0.06$

$1.12 \pm 0.08$

$1.11 \pm 0.09$

$1.41 \pm 0.09$

$1.68 \pm 0.17$
$1.48 \pm 0.13$
$1.73 \pm 0.16$
$1.62 \pm 0.15$
$1.07 \pm 0.11$
$1.41 \pm 0.13$
$1.95 \pm 0.18$
$1.52 \pm 0.14$
$2.25 \pm 0.21$
$1.73 \pm 0.16$
$1.83 \pm 0.17$
$1.72 \pm 0.16$
$2.2 \pm 0.2$
$1.25 \pm 0.11$
$2.36 \pm 0.22$
$1.93 \pm 0.19$
$1.53 \pm 0.14$
$1.57 \pm 0.14$
$1.63 \pm 0.15$
$1.37 \pm 0.13$ 


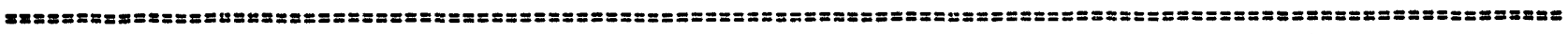
Table C-V. Individual Elemental Concentration in Soils of Cactus Plot $\%$ 10, Saguaro National Monument (cont.)

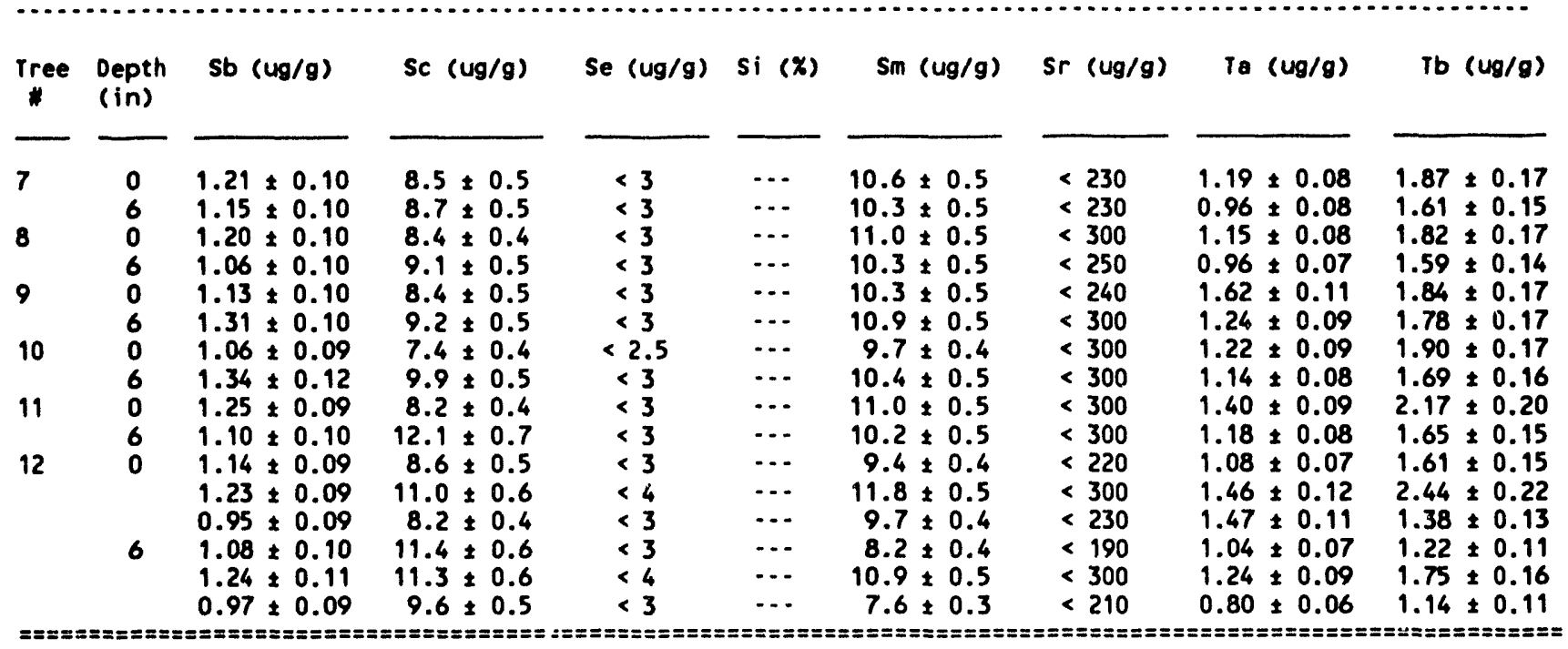

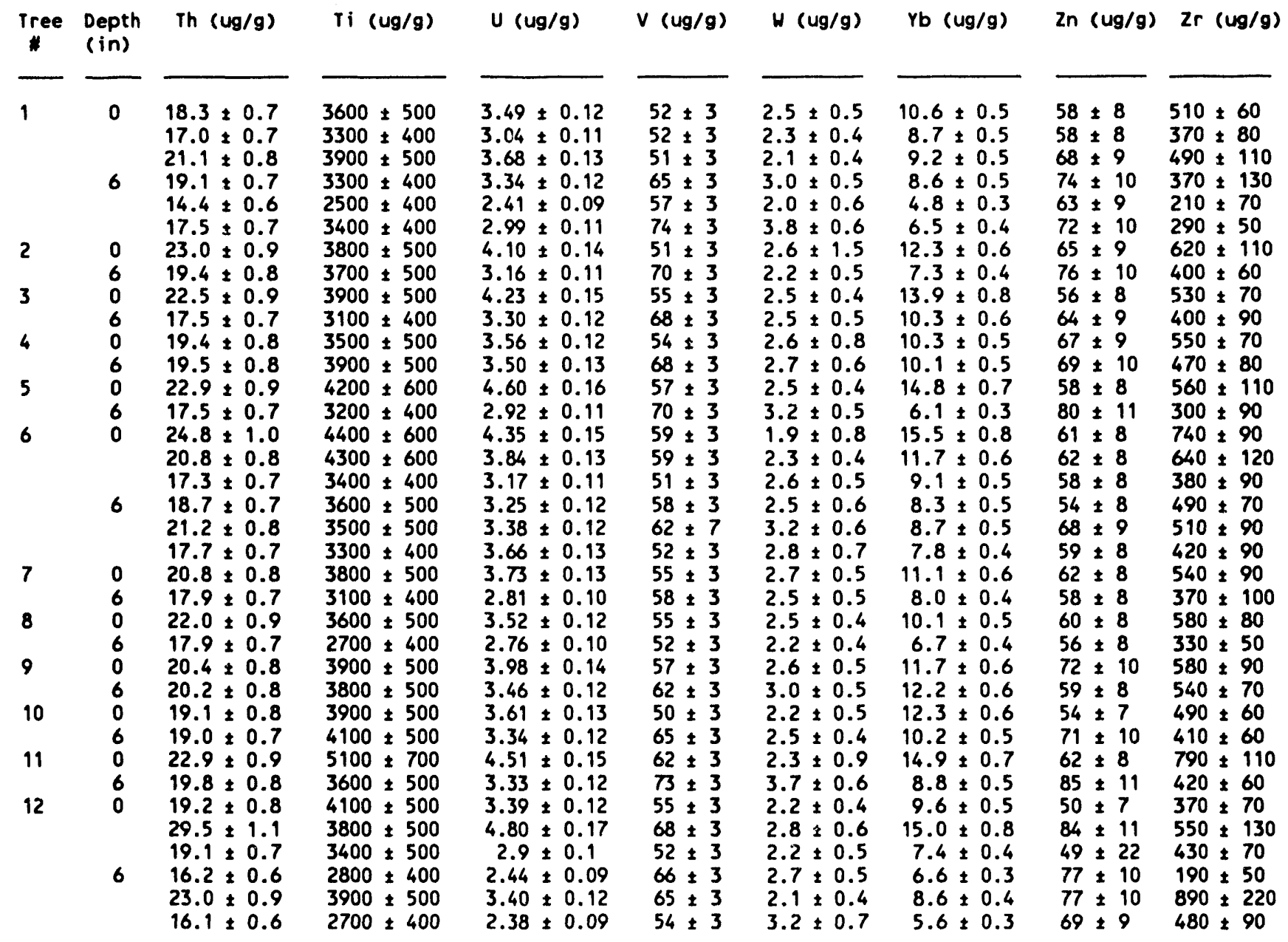




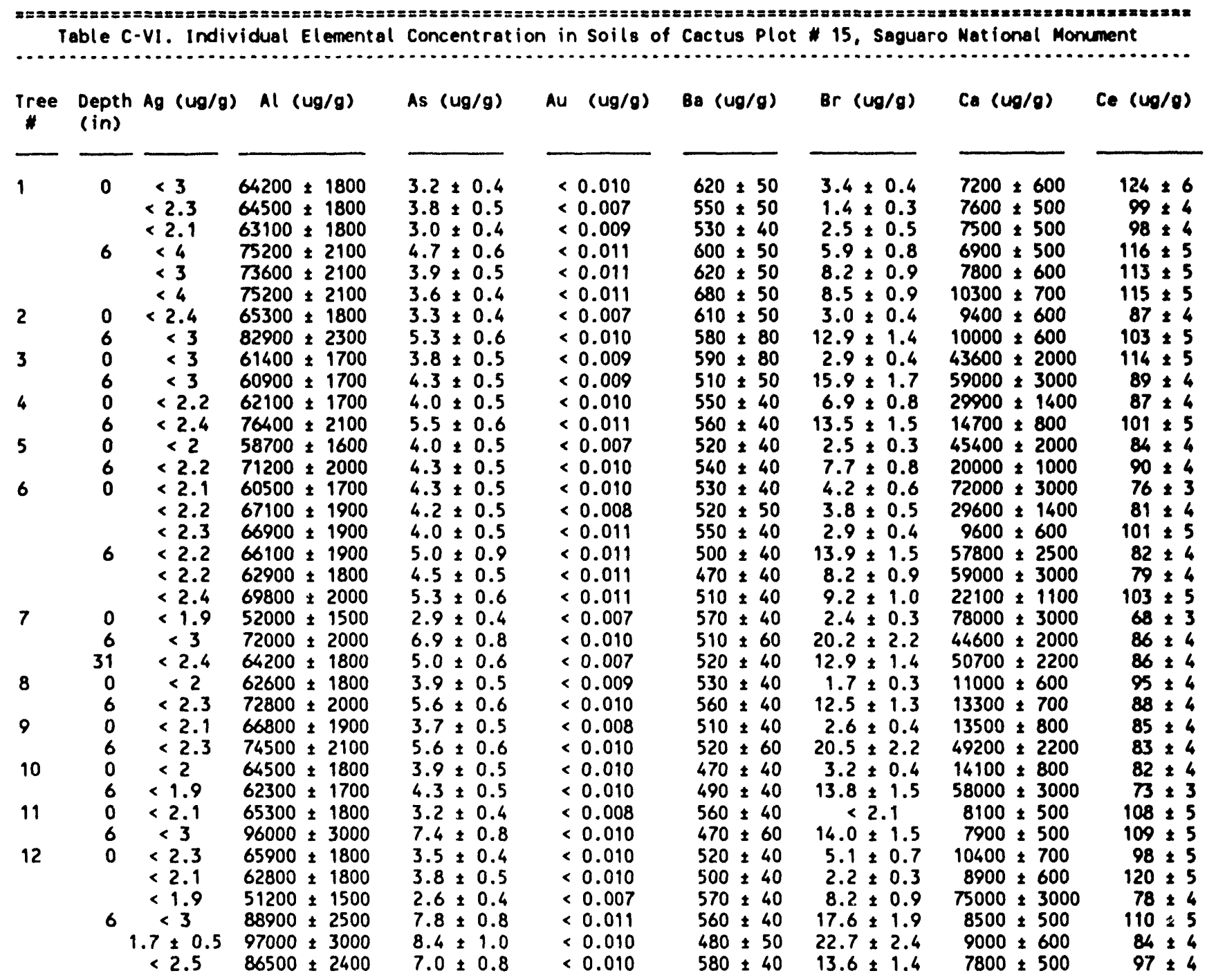




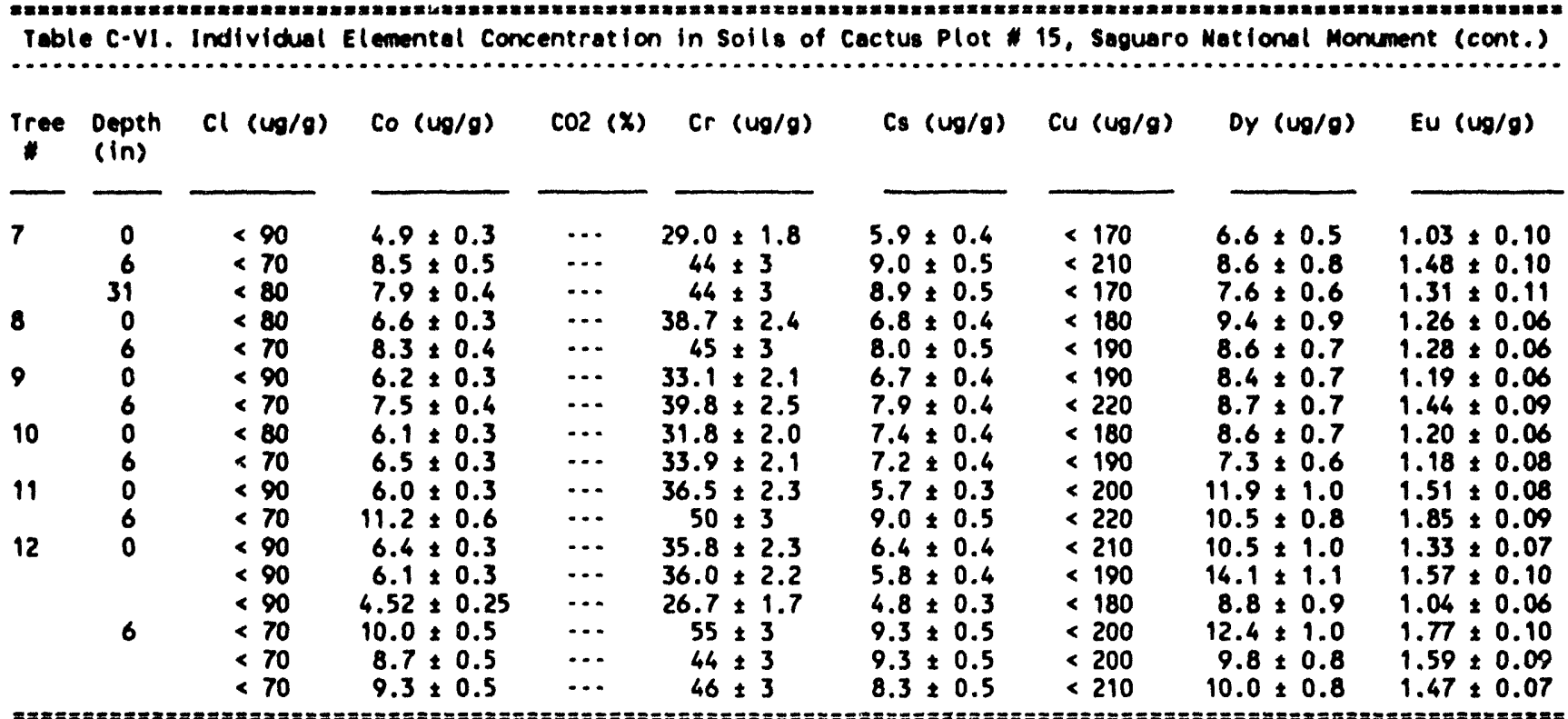

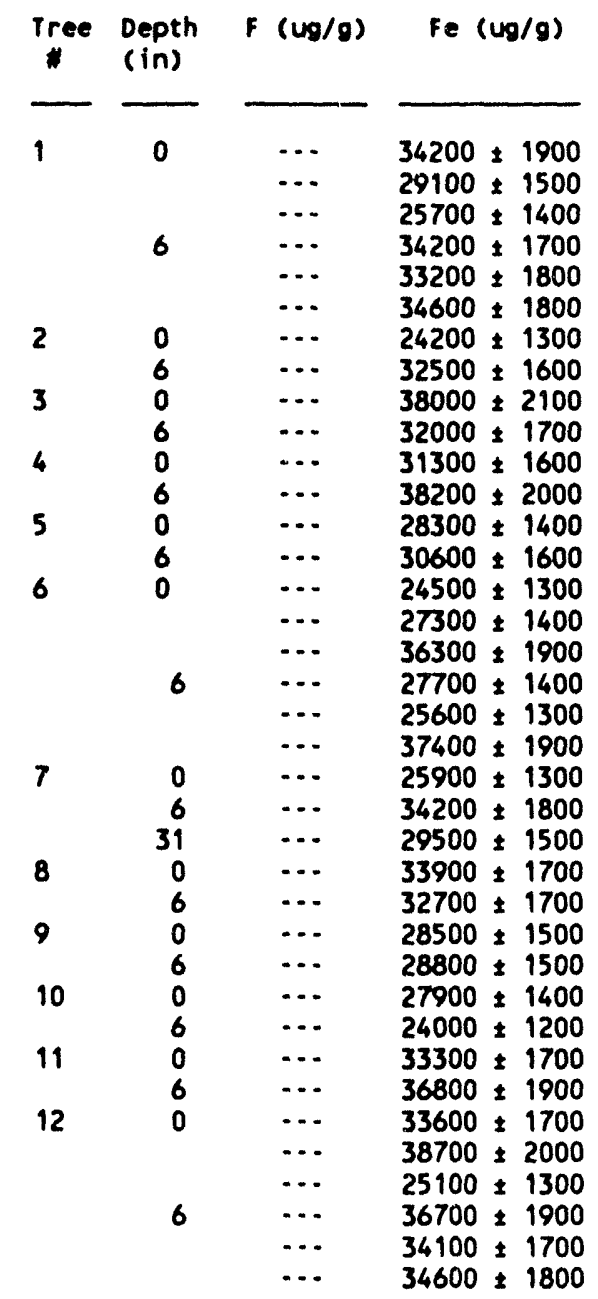

Ga $(u g / g)$

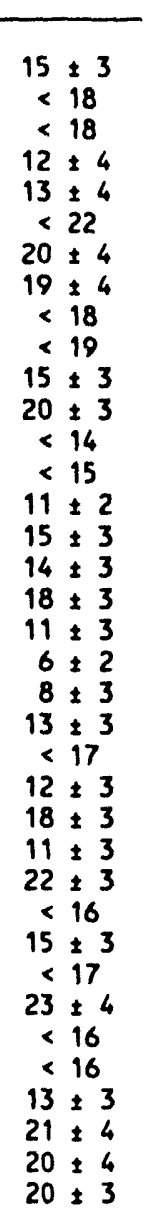

Hf $(u g / g)$

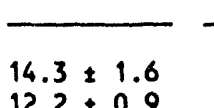

$9.6 \div 0.6$

$10.2 \pm 0.5$

$11.6 \pm 0.7$

$11.5 \div 0.5$

$8.8 \div 0.5$

$7.7 \pm 0.3$

$13.5 \pm 1.5$

$9.8 \pm 1.4$

$10.4 \div 0.7$

$10.7 \pm 1.3$

$11.3 \pm 0.8$

$10.2 \pm 0.6$

$8.6 \div 1.3$

$9.1 \pm 1.1$

$12.5 \pm 0.9$

$8.5 \pm 0.9$

$8.3 \pm 0.9$

$13.4 \pm 0.7$

$11.1 \pm 1.4$

$10.5 \pm 1.1$

$9.2 \pm 0.6$

$14.2 \pm 0.8$

$11.6 \pm 0.6$

$10.3 \pm 0.5$

$7.7 \pm 0.7$

$11.4 \pm 0.7$

$7.6 \pm 0.8$

$16.2 \pm 1.0$

$9.0 \pm 1.1$

$12.6 \pm 0.8$

$17.2 \pm 1.3$

$10.6 \pm 0.7$

$8.9 \pm 0.8$

$6.7 \pm 0.8$

$10.6 \pm 0.5$
$H g(u g / g)$

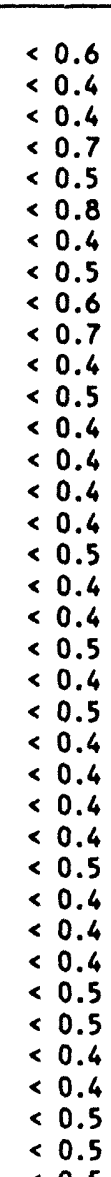

$1(49 / 9)$

$\ln (\log / g)$

K $(u g / g)$
$<0.19$

$<0.17$

$<0.18$

$<0.21$

$<0.17$

$<14$

$4.9 \pm 1.9$

$<14$

$8 \pm 2$

$<16$

$12 \pm 2$

$<17$

$<15$

$<14$

$6.7 \pm 2.0$

$<15$

$<15$

$<15$
$<17$

$10 \pm 2$

$<13$

$<17$

$<13$

$13 \pm 2$

$14 \pm 2$

$<15$

$<15$

$<14$

$13 \pm 2$

$<15$

$13.2 \pm 2.3$

$<15$

$<15$

$<19$

$<17$

$<14$

$<16$

$9.8 \pm 1.9$

$<13$
$<0.21$

$0.12 \pm 0.03$

$<0.18$

$<0.18$

$<0.19$

$<0.19$

$<0.17$

$<0.16$

$<0.18$

$<0.18$

$<0.19$

$<0.19$

$<0.16$

$<0.20$

$<0.15$

$<0.16$

$<0.16$

$<0.17$

$<0.18$

$<0.18$

$<0.16$

$<0.18$

$<0.18$

$<0.18$

$<0.17$

$<0.21$

$<0.19$

$<0.17$

$<0.19$

$<0.19$

$<0.15$
$28200 \pm 1800$ $26400 \pm 1700$ $27500 \pm 1800$ $28100 \div 1900$ $30700 \pm 2000$ $27300 \div 1800$ $27100 \pm 1800$

$30500 \pm 2000$ $25200 \pm 1600$ $23200 \pm 1500$ $27000 \div 1700$ $27300 \pm 1800$ $23500 \div 1500$ $28000 \pm 1800$ $23700 \pm 1500$ $26400 \div 1700$ $30700 \div 2000$ $25100 \div 1600$ $25800+1700$ $27500 \div 1800$ $21300 \div 1400$ $23500 \pm 1500$ $21400 \div 1400$ $28000 \div 1800$ $25500 \pm 1600$ $26000 \pm 1700$ $26300 \pm 1700$ $26600 \pm 1700$ $23500 \pm 1500$ $26000 \pm 1700$ $27000 \pm 1800$ $23400 \pm 2100$ $28100 \pm 2400$ $20500 \div 1400$ $27400 \div 1800$ $27100 \div 1800$ $28100 \pm 1800$ 


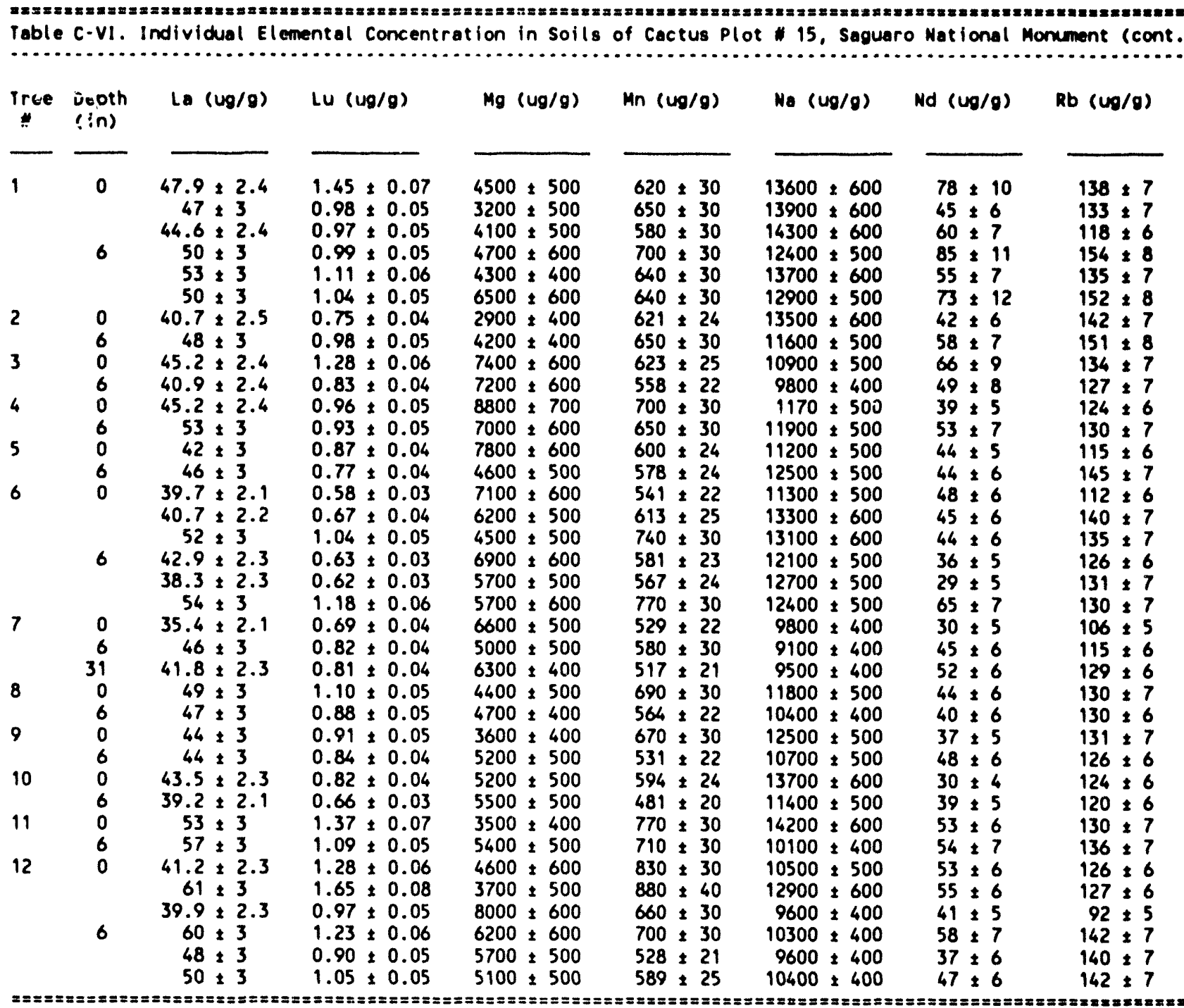

\begin{tabular}{|c|c|c|c|c|c|c|c|c|c|}
\hline$\underset{*}{\text { Tree }}$ & $\begin{array}{l}\text { Depth } \\
\text { (in) }\end{array}$ & Sb $(u g / g)$ & Sc $(u g / g)$ & Se $(u g / g)$ & si $(x)$ & $\mathrm{Sm}(u g / g)$ & $\operatorname{Sr}(u g / g)$ & To (ug/g) & Tb $(u g / g)$ \\
\hline \multirow[t]{5}{*}{1} & 0 & $1.09 \pm 0.09$ & $9.00 \pm 0.5$ & $\because$ & 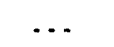 & $9.2 \pm 0.4$ & $<300$ & $1.27 \div 0.10$ & $2.04 \div 0.20$ \\
\hline & & $\begin{array}{l}1.21 \div 0.09 \\
1.10 \div 0.08\end{array}$ & $7.6 \div 0.4$ & $<2.3$ & $\cdots$ & $9.0 \pm 0.4$ & $<200$ & $1.25 \div 0.09$ & $1.32 \div 0.12$ \\
\hline & 6 & $1.09 \pm 0.10$ & $10.8 \div 0.6$ & $<3$ & $\cdots$ & $\begin{array}{l}8.4 \pm 0.4 \\
9.7\end{array}$ & 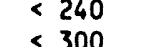 & $1.00 \pm 0.08$ & $1.17 \div 0.10$ \\
\hline & & $1.34 \pm 0.10$ & $9.8 \div 0.5$ & $<3$ & & $9.4 \div 0.4$ & $<230$ & $1.08 \div 0.07$ & $1.48 \pm 0.14$ \\
\hline & & $1.21 \div 0.10$ & $10.6 \div 0.6$ & $<4$ & 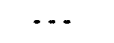 & $9.9 \div 0.5$ & $<300$ & $1.22 \div 0.09$ & $1.58 \div 0.15$ \\
\hline \multirow[t]{2}{*}{2} & 0 & $1.08 \div 0.09$ & $7.4 \pm 0.4$ & $<2.4$ & $\cdots$ & $7.8 \pm 0.4$ & $<210$ & $1.15 \div 0.08$ & $1.03 \div 0.09$ \\
\hline & 6 & $1.12 \pm 0.10$ & $11.2 \pm 0.6$ & $<3$ & $\cdots$ & $8.9 \pm 0.4$ & $<220$ & $1.10 \pm 0$ & $1.50 \div 0.14$ \\
\hline \multirow[t]{2}{*}{3} & 0 & $1.23: 0.09$ & $9.6 \pm 0.5$ & $<4$ & $\cdots$ & $8.6 \div 0.4$ & $<300$ & $1.41 \pm 0.11$ & $1.64 \pm 0.20$ \\
\hline & 6 & $1.11 \pm 0.10$ & $8.8 \pm 0.5$ & $<4$ & $\cdots$ & $7.9 \pm 0.4$ & $<240$ & $1.26 \pm 0.08$ & $1.11 \div 0.10$ \\
\hline \multirow[t]{2}{*}{4} & 0 & $1.25 \pm 0.10$ & $8.1 \pm 0.4$ & $<3$ & $\cdots$ & $8.3 \div 0.4$ & $<300$ & $1.21 \times 0.09$ & $1.40 \pm 0.13$ \\
\hline & 6 & $1.42 \pm 0.12$ & $10.5 \pm 0.6$ & $<3$ & $\cdots$ & $10.5 \div 0.5$ & $<300$ & $1.39 \pm 0.09$ & $1.39 \div 0.13$ \\
\hline \multirow[t]{2}{*}{5} & 0 & $1.33 \div 0.10$ & $7.5 \pm 0$ & $<3$ & $\cdots$ & $8.0 \pm 0.4$ & $280 \pm 70$ & $1.18 \div 0.08$ & $1.23 \div 0.11$ \\
\hline & 6 & $1.31 \pm 0.11$ & $8.8 \div 0.5$ & $<3$ & $\cdots$ & $8.0 \pm 0.4$ & $<220$ & $1.14 \pm 0.08$ & $1.28 \div 0.12$ \\
\hline \multirow[t]{6}{*}{6} & 0 & $1.25 \pm 0.10$ & $7.7 \pm 0.4$ & $<3$ & $\cdots$ & $7.5 \pm 0.3$ & $210 \pm 50$ & $0.99 \pm 0.07$ & $1.00 \div 0.09$ \\
\hline & & $139+01$ & $8.3 \pm 0.4$ & $<3$ & $\cdots$ & $7.3 \pm 0.3$ & $<220$ & $1.13 \pm 0.08$ & $1.04=0.10$ \\
\hline & & & $9.0 \pm 0$. & $<3$ & $\cdots$ & $10.1 \pm 0.5$ & $<300$ & $1.43 \pm 0.09$ & $1.53 \div 0.14$ \\
\hline & 6 & $1.50 \pm 0.12$ & $8.6 \pm 0.5$ & $<3$ & $\cdots$ & $8.2 \pm 0.4$ & $330 \pm 80$ & $1.11 \pm 0.08$ & $1.05 \pm 0.10$ \\
\hline & & $1.23 \pm 0.10$ & $8.3 \pm 0$. & $<3$ & $\cdots$ & $6.8 \pm 0.3$ & $<210$ & $1.20 \div 0.08$ & $1.08 \div 0.10$ \\
\hline & & $1.67 \pm 0.13$ & $9.8 \div 0.5$ & $<3$ & $\cdots$ & $10.7 \pm 0.5$ & $<300$ & $1.38 \pm 0.09$ & $1.58 \div 0.15$ \\
\hline
\end{tabular}




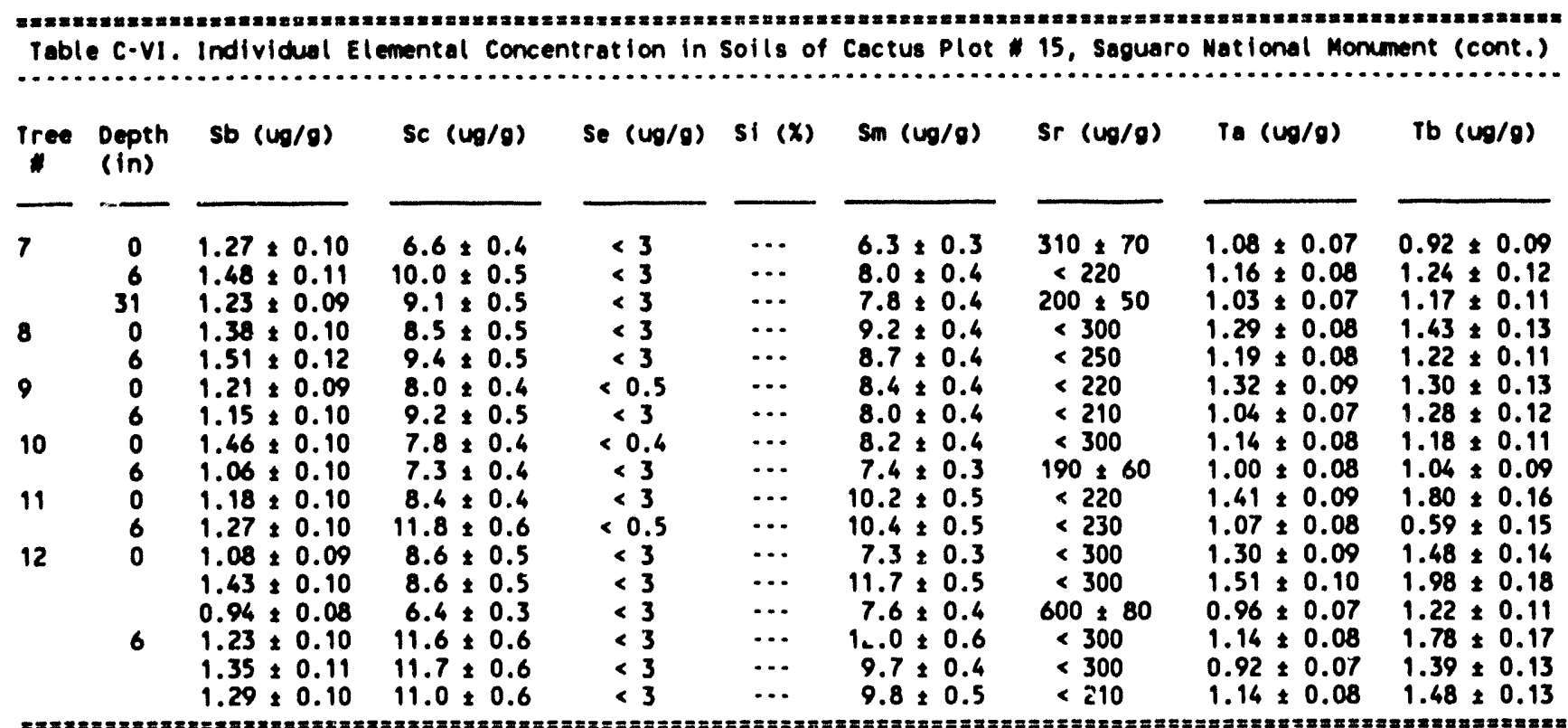

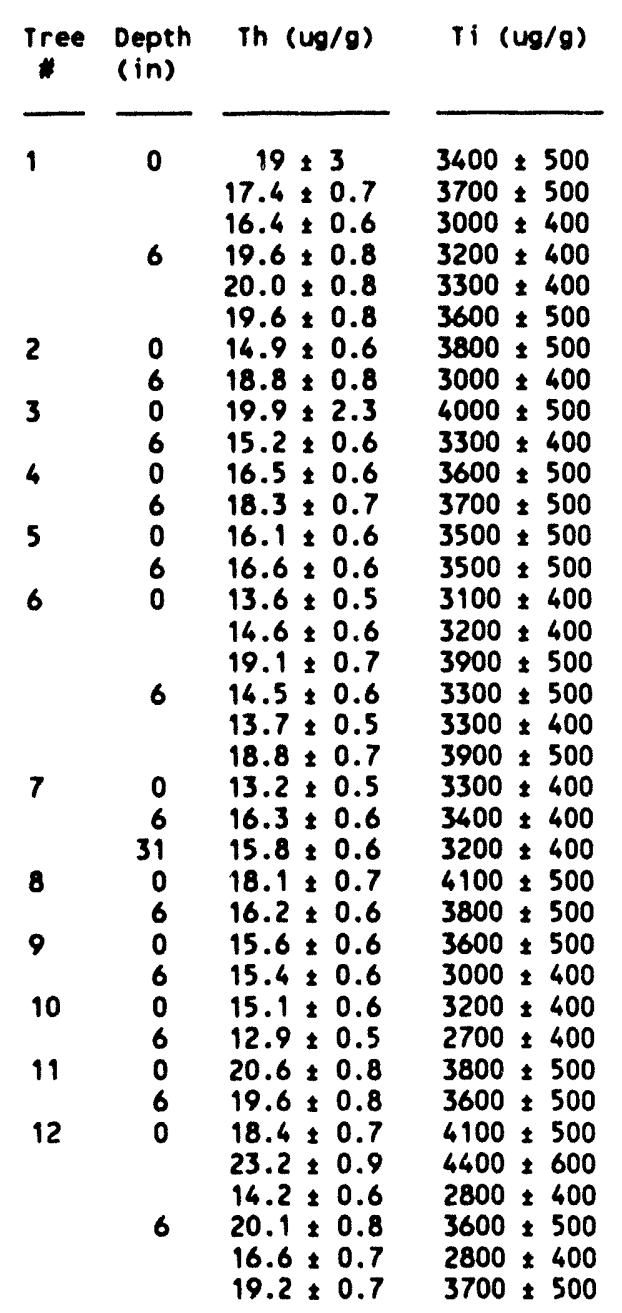

$U(u g / g)$

$\overline{3.44 \pm 0.12}$

$3.23 \pm 0.11$

$2.91 \div 0.10$

$3.20 \div 0.12$

$3.27 \pm 0.12$

$3.19 \div 0.12$

$2.76 \div 0.10$

$3.04 \div 0.11$

$3.45 \div 0.12$

$2.84 \pm 0.10$

$3.21 \div 0.11$

$3.36 \div 0.12$

$3.11 \div 0.11$

$3.07 \pm 0.11$

$2.69 \div 0.10$

$2.94 \div 0.11$

$3.39 \pm 0.12$

$2.86 \div 0.10$

$3.02 \div 0.11$

$3.72 \pm 0.13$

$2.71 \pm 0.10$

$3.10 \pm 0.11$

$3.07 \div 0.11$

$3.56 \div 0.12$

$3.39 \pm 0.12$

$3.56 \div 0.12$

$2.74 \div 0.10$

$3.26+0.11$

$2.92 \div 0.11$

$3.87 \div 0.14$

$3.14 \pm 0.11$

$3.25 \pm 0.12$

$4.18 \div 0.15$

$2.7 \pm 0.1$

$3.65 \div 0.13$

$2.75 \pm 0.10$

$3.31 \div 0.12$
$V(u g / g)$

$W(u g / g)$

$\begin{aligned} 56 & \pm 3 \\ 54 & \pm 3 \\ 45.9 & \pm 2.3 \\ 61 & \pm 3 \\ 58 & \pm 3 \\ 60 & \pm 3\end{aligned}$

$2.6 \pm 0.5$

$2.3 \pm 0.4$

$2.4 \pm 0.5$

$2.9 \div 0.6$

$3.2 \div 1.4$

$42.1 \pm 2.32 .3 \pm 0.4$

$63 \pm 3 \quad 2.7 \pm 0.5$

$56 \pm 3 \quad 2.4 \pm 0.6$

$56 \pm 3 \quad 2.8 \pm 0.5$

$55 \pm 3 \quad 2.9 \pm 1.1$

$71 \pm 3 \quad 2.7 \pm 0.5$

$52 \pm 3 \quad 2.4 \pm 0.4$

$53 \pm 3 \quad 3.0 \pm 0.6$

$44 \pm 2 \quad 1.8 \pm 0.3$

$53 \pm 3 \quad 3.4 \pm 0.6$

$62 \pm 3 \quad 3.0 \pm 0.6$

$56 \pm 6$

$49 \div 5$

$68 \pm 3$

$46 \pm 2$

$69 \div 3$

$55 \pm 3$

$59+3$

$68 \pm 3$

$50 \pm 2$

$53 \pm 3$

$52 \pm 3$

$44.7 \pm 2.3$

$57 \pm 3$

$71 \pm 3$

$2.0 \div 0.4$

$2.3 \div 0.4$

$3.4 \div 0.5$

$2.9 \pm 0.7$

$3.0 \pm 0.5$

$2.5 \neq 0.5$

$2.6 \pm 0.6$

$2.6 \pm 0.4$

$2.1 \pm 0.5$

$3.2 \div 0.5$

$2.6 \pm 0.7$

$55 \pm 3$

$3.0 \div 0.5$

$2.2 \div 0.4$

$62 \pm 3$

$4.1 \pm 0.7$

$3 \quad 2.7 \pm 0.5$

$45.6 \pm 2.32 .0 \pm 0.4$

$70 \pm 6 \quad 3.3 \pm 0.6$

$\begin{array}{ll}66 \pm 3 & 2.1 \pm 0.5 \\ 70 \div 3 & 3.2 \pm 0.6\end{array}$
Yb $(u g / g)$

Zn $(u g / g)$

$\operatorname{2r}(u g / g)$

$8.7 \pm 0.5$

$7.1 \pm 0.4$

$6.5 \pm 0.3$

$7.1 \div 0.4$

$7.1 \pm 0.4$

$7.3 \pm 0.4$

$5.4 \pm 0.3$

$6.8 \div 0.4$

$7.1 \pm 0.4$

$5.4 \pm 0.3$

$8.0 \pm 0.4$

$7.9 \pm 0.4$

$6.8 \div 0.4$

$6.2 \div 0.3$

$4.6 \pm 0.3$

$5.3 \div 0.3$

$8.3 \pm 0.4$

$5.4 \pm 0.4$

$4.7 \pm 0.3$

$9.2 \pm 0.5$

$5.3 \div 0.3$

$6.5 \div 0.4$

$5.1 \pm 0.3$

$8.5 \div 0.4$

$6.8 \pm 0.4$

$6.8 \pm 0.4$

$7.0 \div 0.4$

$6.5 \div 0.3$

$5.2 \div 0.3$

$10.4 \div 0.6$

$8.1 \pm 0.4$

$8.0 \pm 0.4$

$13.1 \div 0.7$

$7.6 \div 0.4$

$10.5 \div 0.6$

$6.9 \div 0.4$

$7.9 \div 0.4$
$72 \pm 10$

$42 \div 17$

$58 \pm 8$

$75 \div 10$

$70 \pm 9$

$71 \div 10$

$46 \pm 20$

$75 \pm 10$

$76 \pm 10$

$56 \pm 8$

$61 \pm 8$

$66 \pm 9$

$56 \pm 8$

$61 \pm 8$

$58 \pm 8$

$63 \pm 9$

$57 \pm 8$

$56 \div 8$

$53 \pm 7$

$60 \div 8$

$47 \pm 7$

$62 \div 9$

$65 \pm 9$

$62 \pm 8$

$54 \div 8$

$66 \pm 9$

$59 \pm 8$

$60 \div 8$

$50 \div 7$

$62 \pm 9$

$72 \pm 10$

$66 \div 9$

$60 \pm 8$

$57 \pm 8$

$78 \div 10$

$77 \pm 10$

$68 \pm 9$
$460 \div 60$

$380 \pm 50$

$600 \pm 110$

$760 \div 110$

$740 \pm 110$

$300 \pm 50$

$530 \pm 90$

$380 \div 50$

$610 \div 90$

$460 \pm 60$

$420 \pm 60$

$470 \pm 130$

$420 \pm 100$

$340 \pm 50$

$360 \pm 90$

$510 \pm 70$

$350 \div 70$

$330 \pm 50$

$560 \div 80$

$480 \div 60$

$420 \pm 60$

$690=110$

$600 \div 70$

$420+60$

$460 \div 70$

$310 \div 50$

$400 \div 60$

$260 \pm 70$

$570 \pm 90$

$300+80$

$490 \div 80$

$780 \div 120$

$420 \pm 60$

$280 \div 110$

$240 \pm 50$

$490 \pm 70$
$330 \pm 60$ 


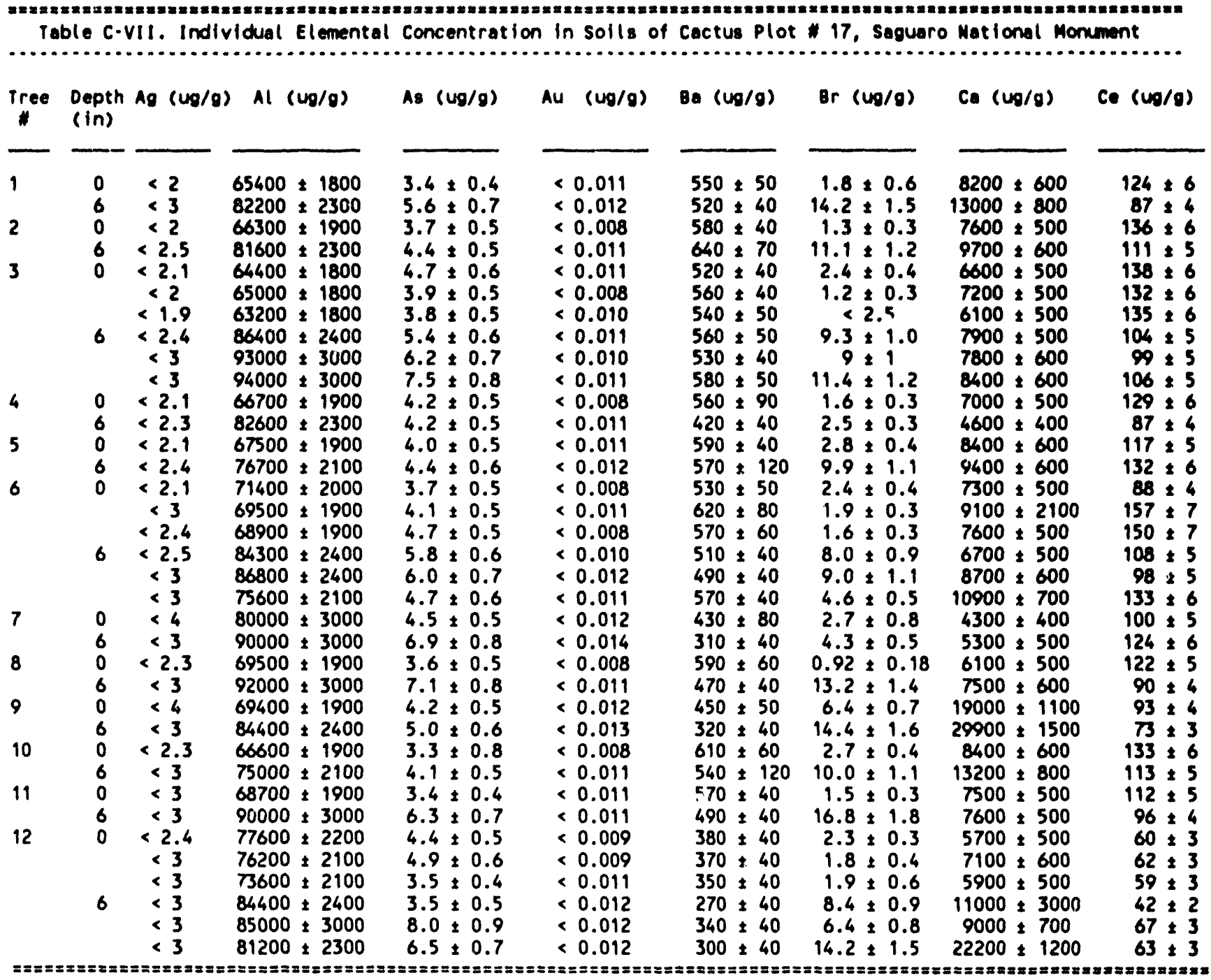

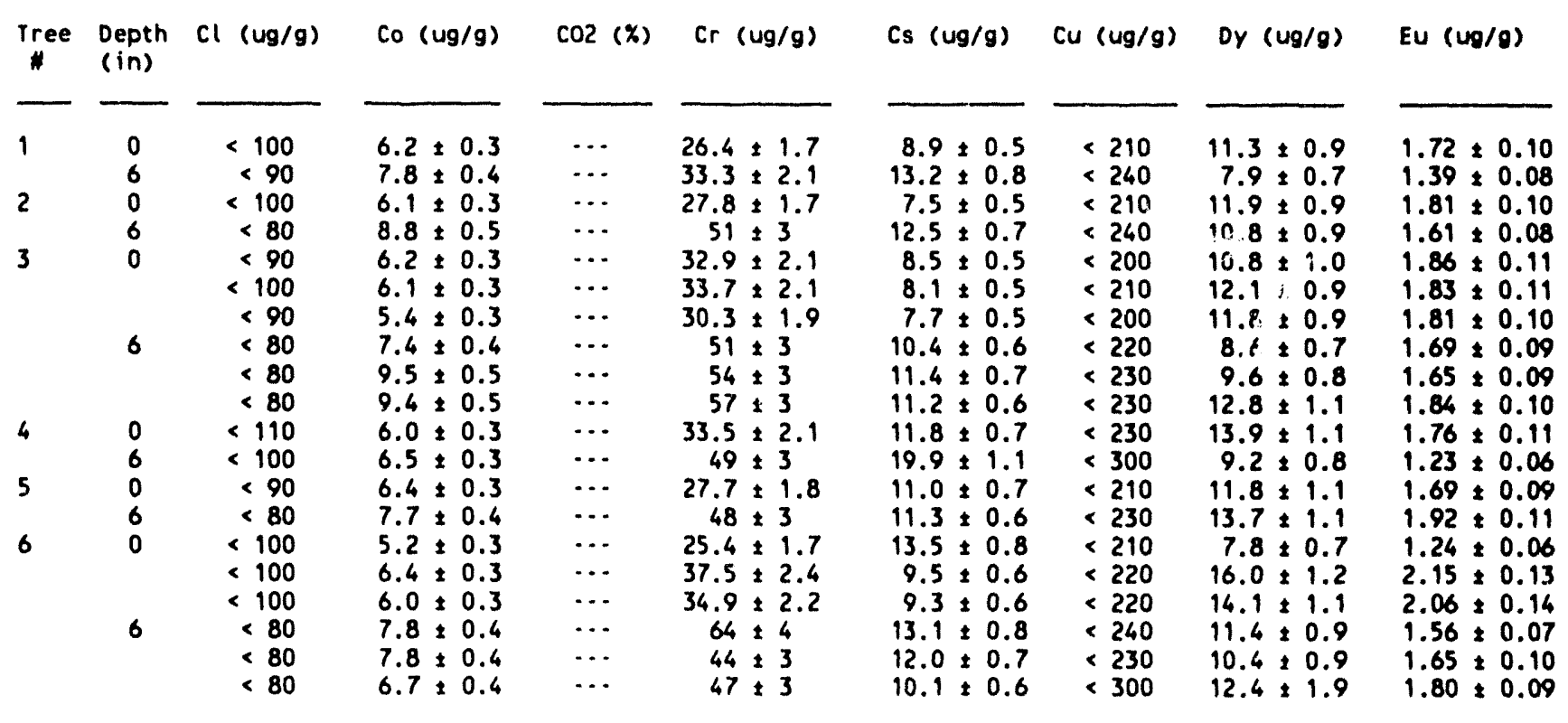




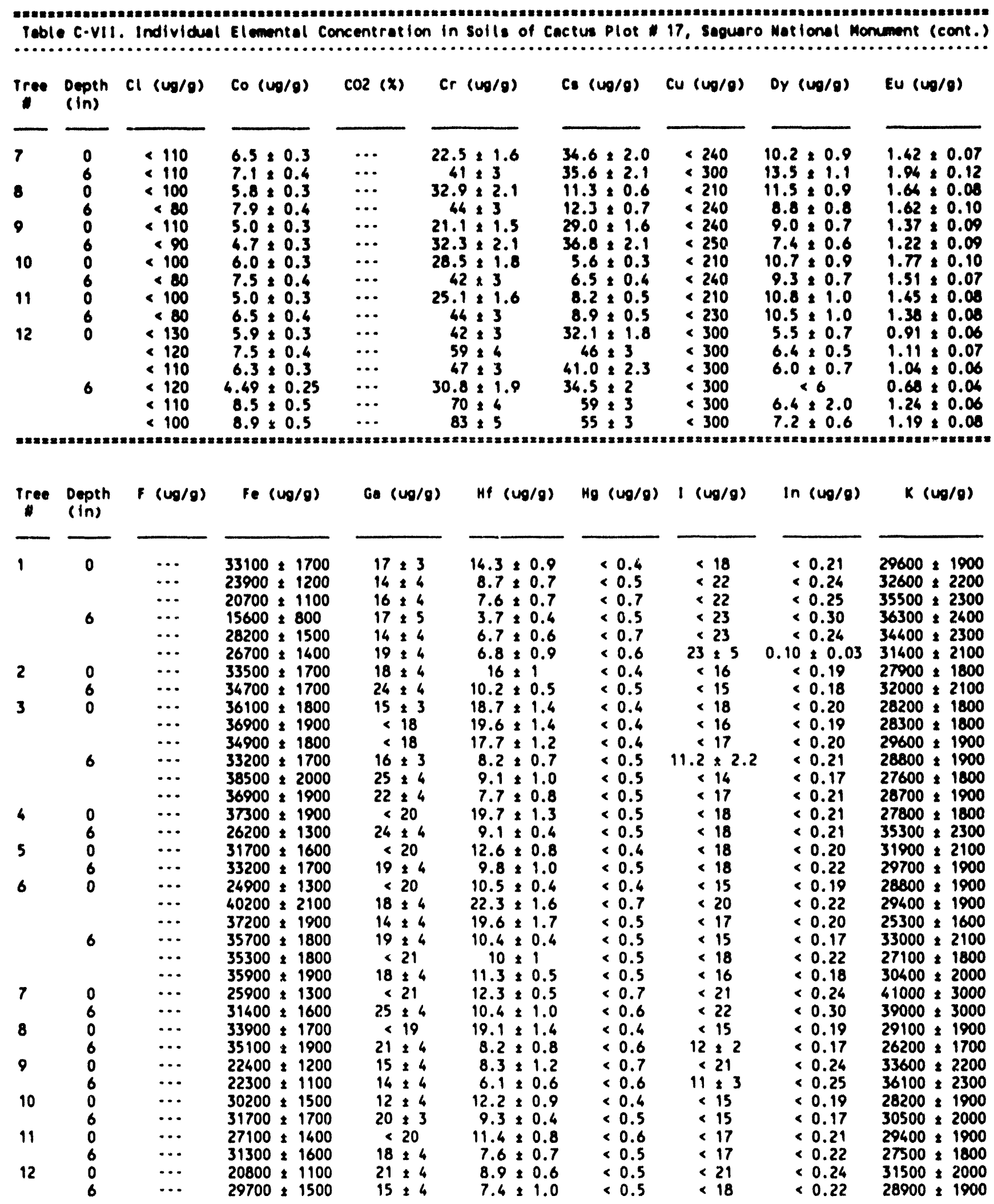




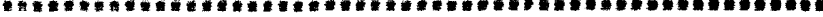

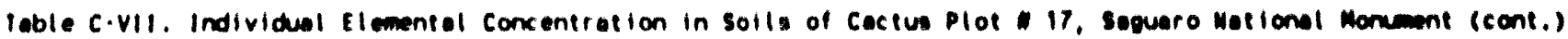

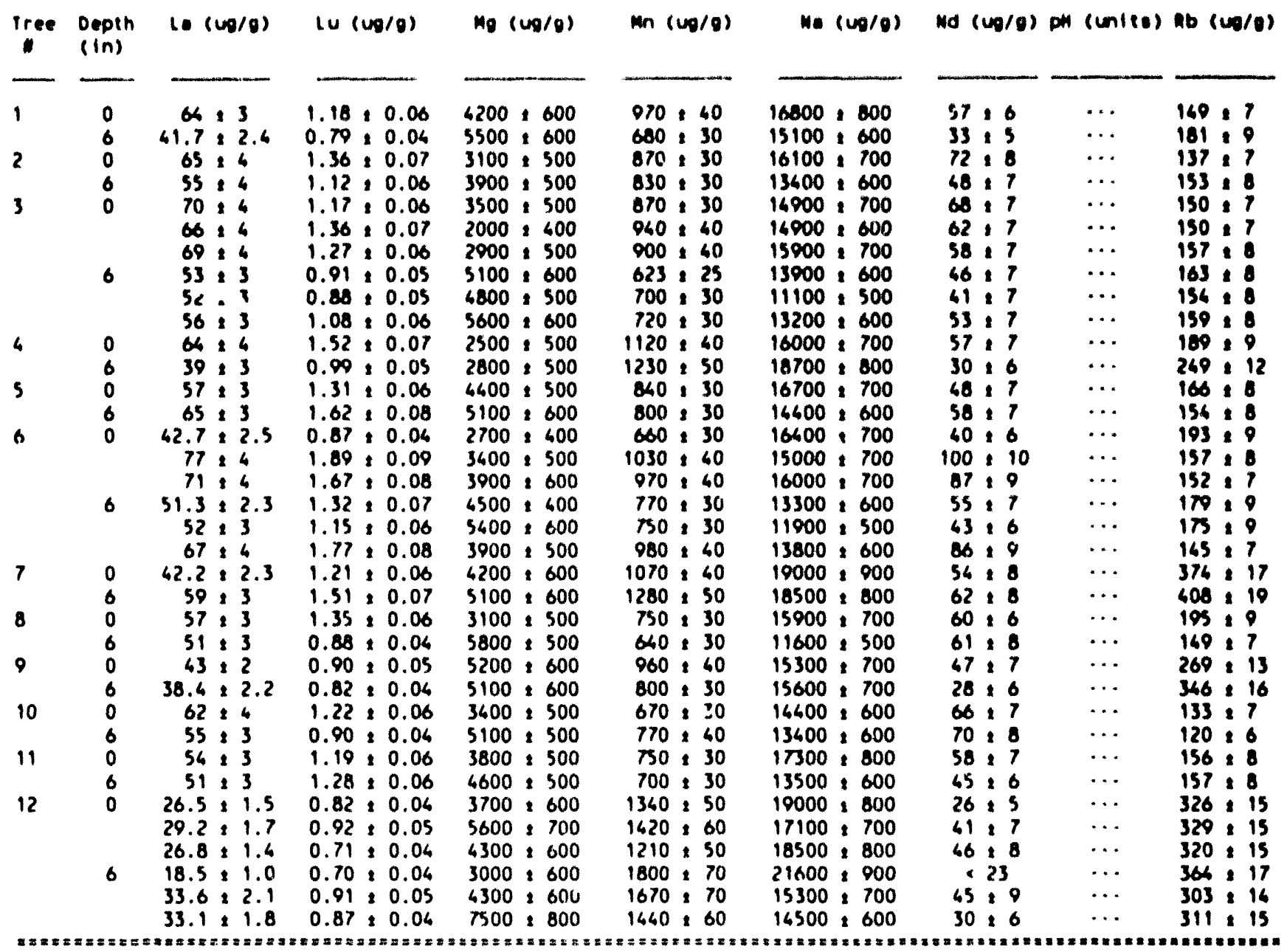

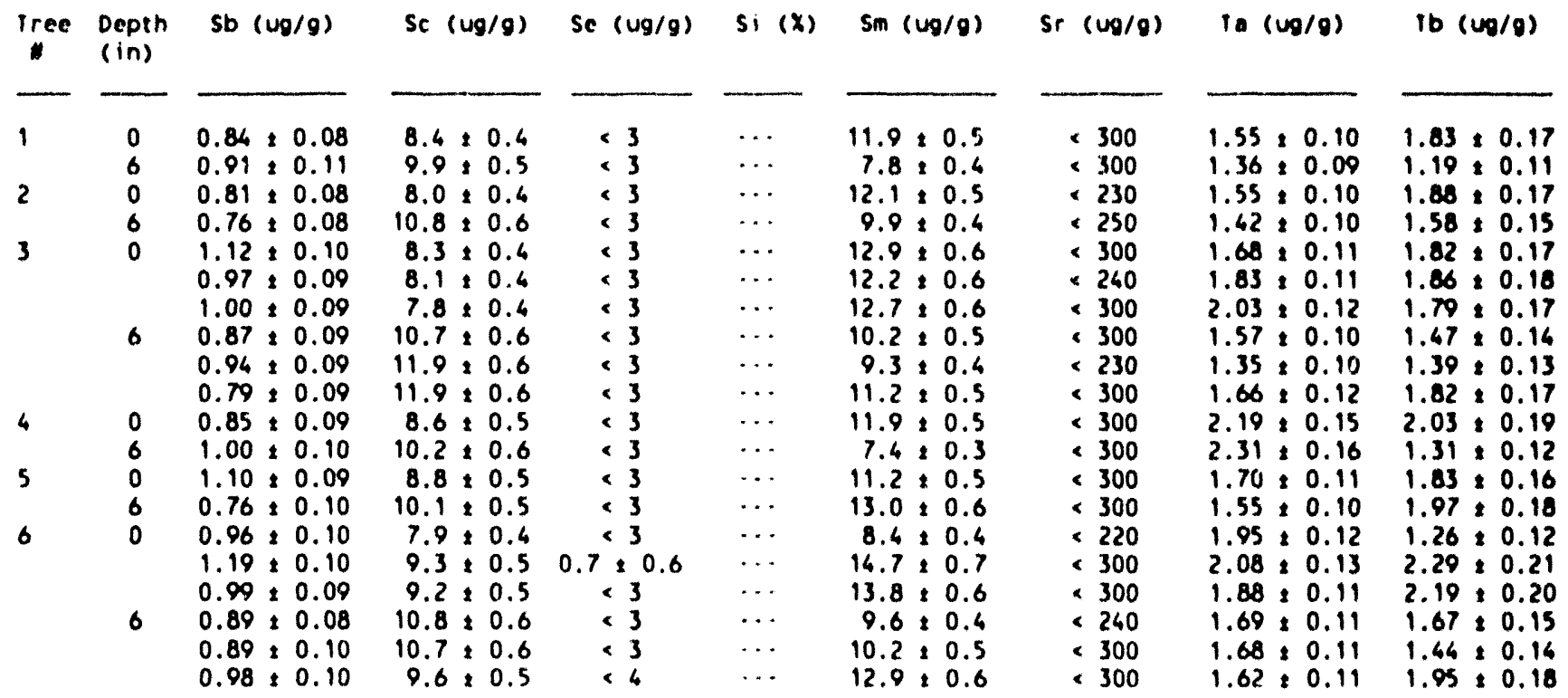




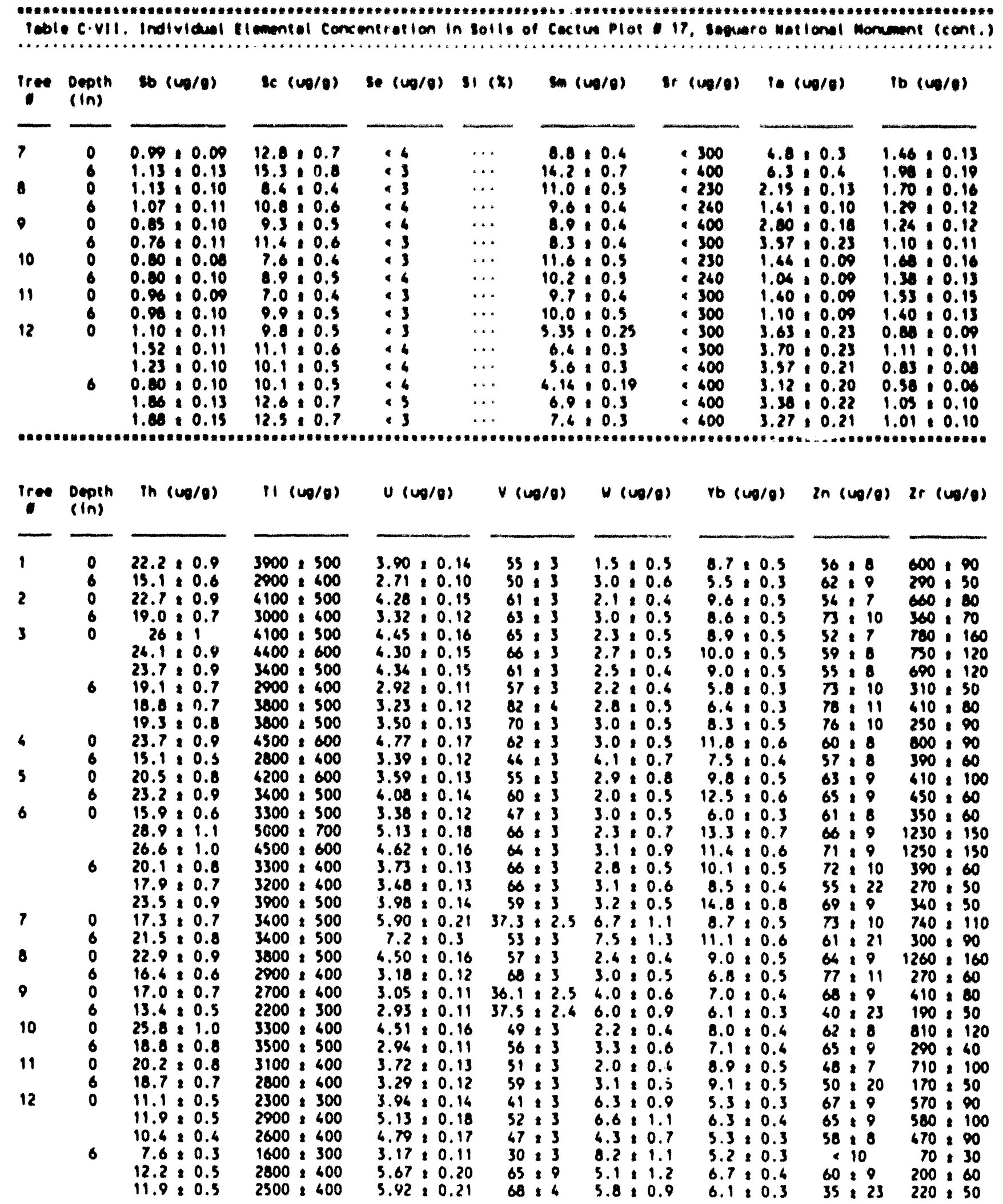




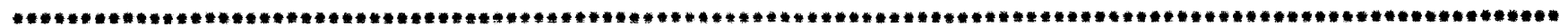

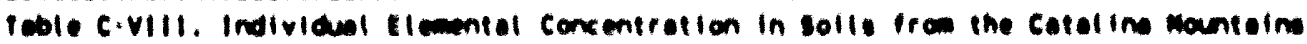

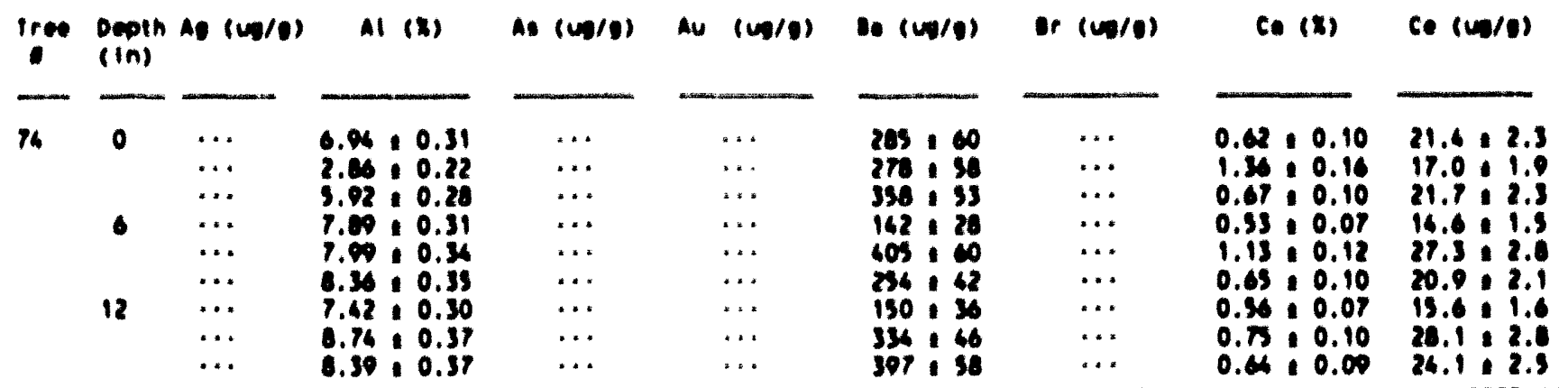

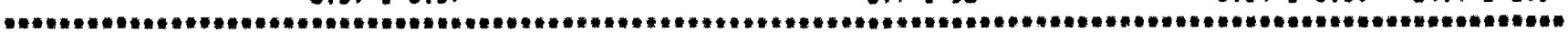

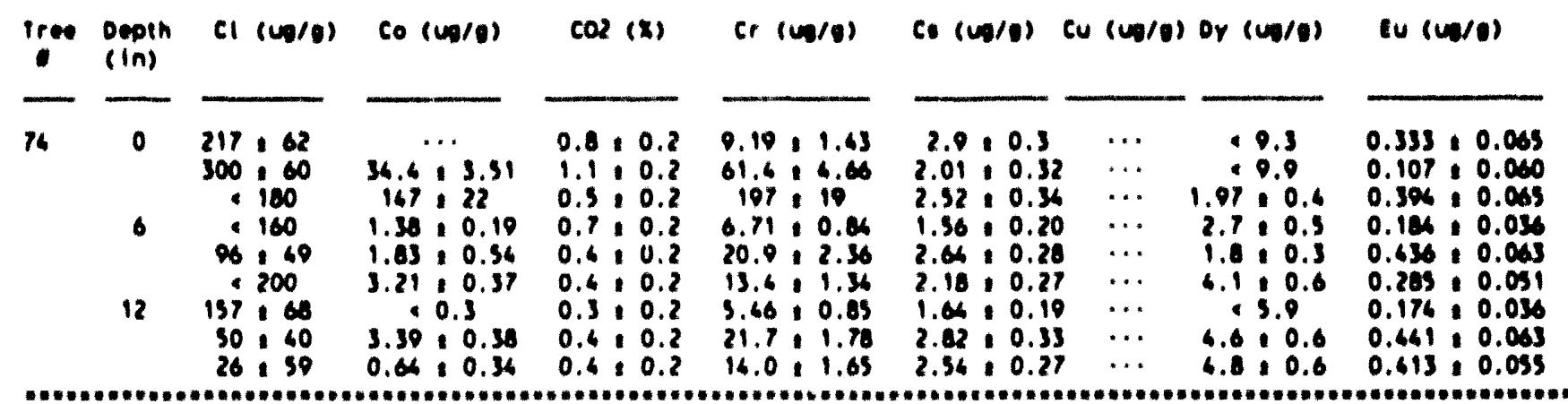

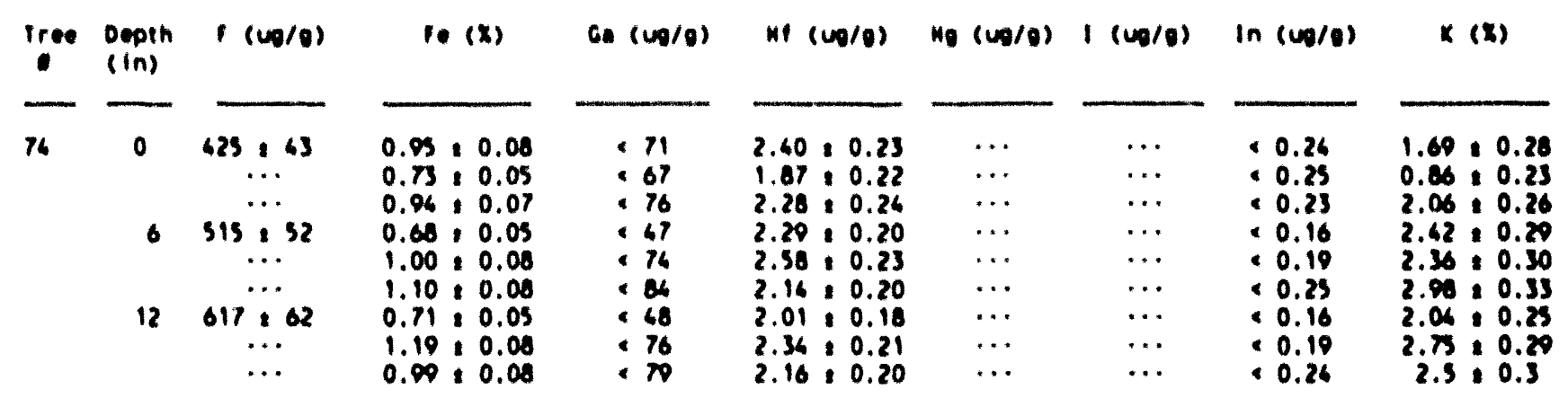




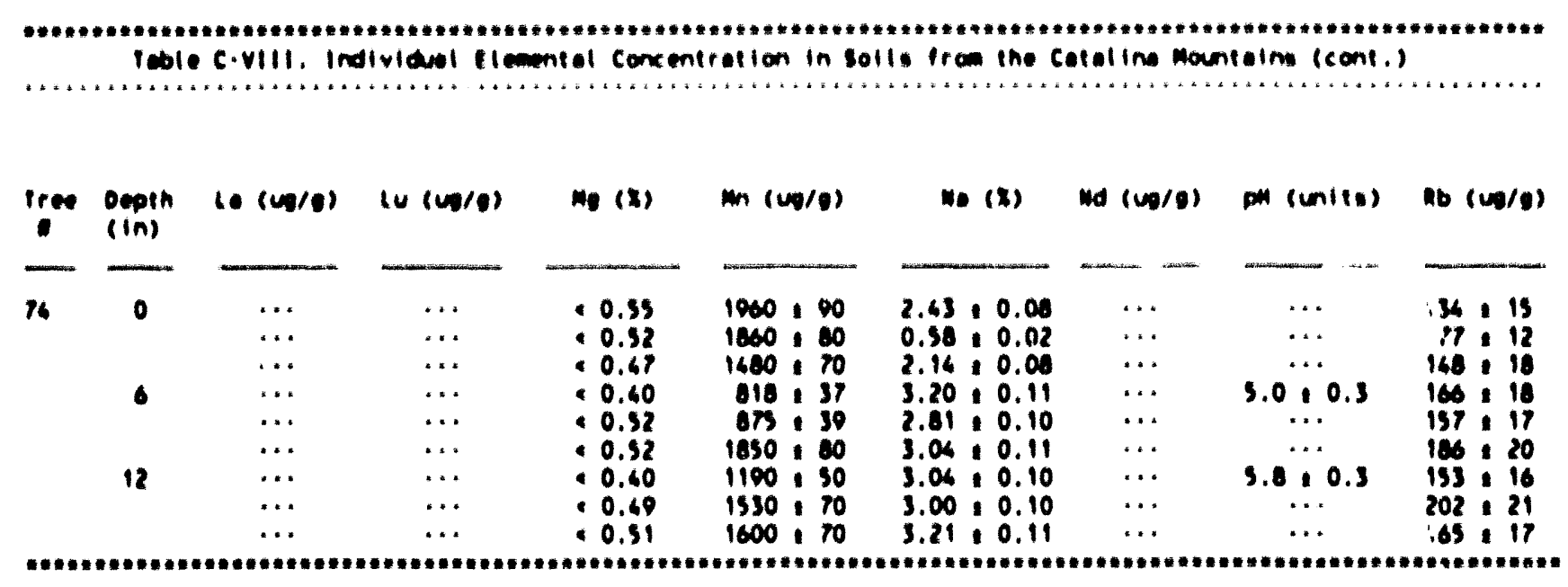

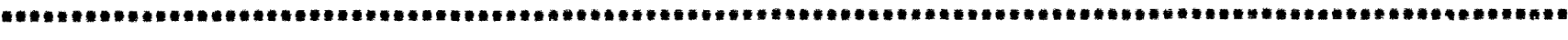

\begin{tabular}{|c|c|c|c|c|c|c|c|c|c|}
\hline Iree & $\begin{array}{l}\text { Depln } \\
\text { (In) }\end{array}$ & $s b(v 0 / \theta)$ & sc $\left(\omega_{0} / \theta\right)$ & $80(\omega 0 / 0)$ & $11(8)$ & $\ln (\omega 0 / 0)$ & ir $(\omega 0 / \theta)$ & 1. $(\omega / 0)$ & ib $(00 / 0)$ \\
\hline 76 & 12 & $\begin{array}{l:l}0.89: 0.23 \\
0.87: 0.25 \\
1.03: 0.26 \\
0.61: 0.11 \\
0.57: 0.18 \\
0.36: 0.15 \\
0.20: 0.11 \\
0.52: 0.15 \\
0.20: 0.15\end{array}$ & $\begin{array}{l:l}3.63: 0.25 \\
2.35: 0.12 \\
3.29: 0.23 \\
2.60: 0.13 \\
6.15: 0.29 \\
6.21: 0.21 \\
2.80: 0.20 \\
4.00: 0.26 \\
6.26: 0.30\end{array}$ & $\begin{array}{l}\ldots \\
\ldots \\
\ldots \\
\ldots \\
\ldots \\
\ldots \\
\ldots\end{array}$ & $\begin{array}{r:l}27.1 & 1.8 \\
9.6 & 0.6 \\
23.9 & 1.9 \\
28 & ? \\
27.1 & 1.7 \\
20 & 3 \\
29.4 & 1.5 \\
28.6 & 1.4 \\
32.5 & 1.6\end{array}$ & $\begin{array}{l:l}2.97 & 0.56 \\
2.11 & 0.42 \\
1.03 & 0.61 \\
1.92 & 0.26 \\
3.16 & 0.56 \\
2.66 & 0.33 \\
2.76 & 0.45 \\
2.37 & 0.33 \\
3.56 & 0.63\end{array}$ & $\begin{array}{r}680 \\
480 \\
330 \\
270 \\
370 \\
360 \\
300 \\
390 \\
330\end{array}$ & $\begin{array}{l:l}1.69 & 0.30 \\
0.67 & 0.16 \\
3.00 & 0.63 \\
1.86 & 0.20 \\
1.56 & 0.27 \\
2.06 & 0.23 \\
2.00 & 0.33 \\
1.07 & 0.22 \\
1.70 & 0.27\end{array}$ & $\begin{array}{l}\ldots \\
\ldots \\
\ldots \\
\ldots \\
\ldots \\
\ldots \\
\ldots\end{array}$ \\
\hline
\end{tabular}

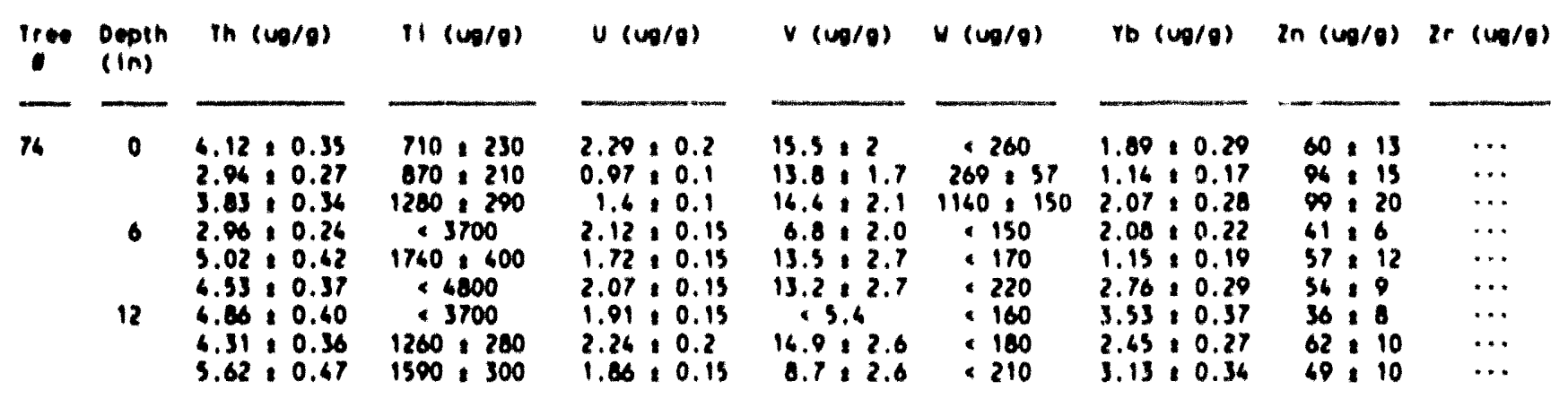




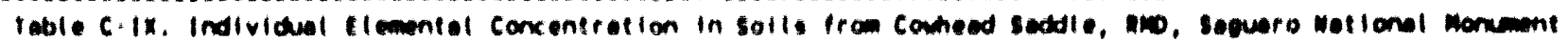

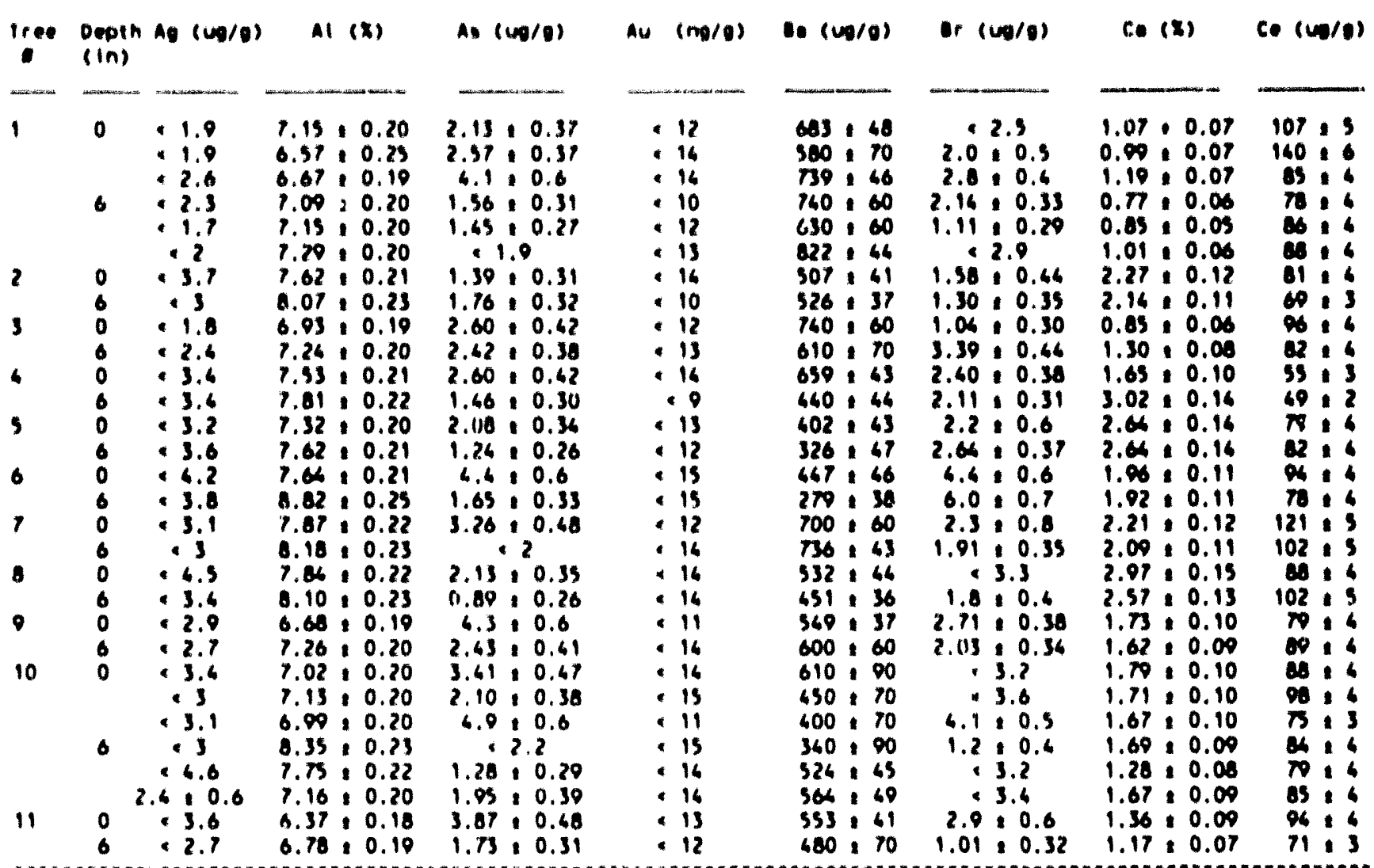

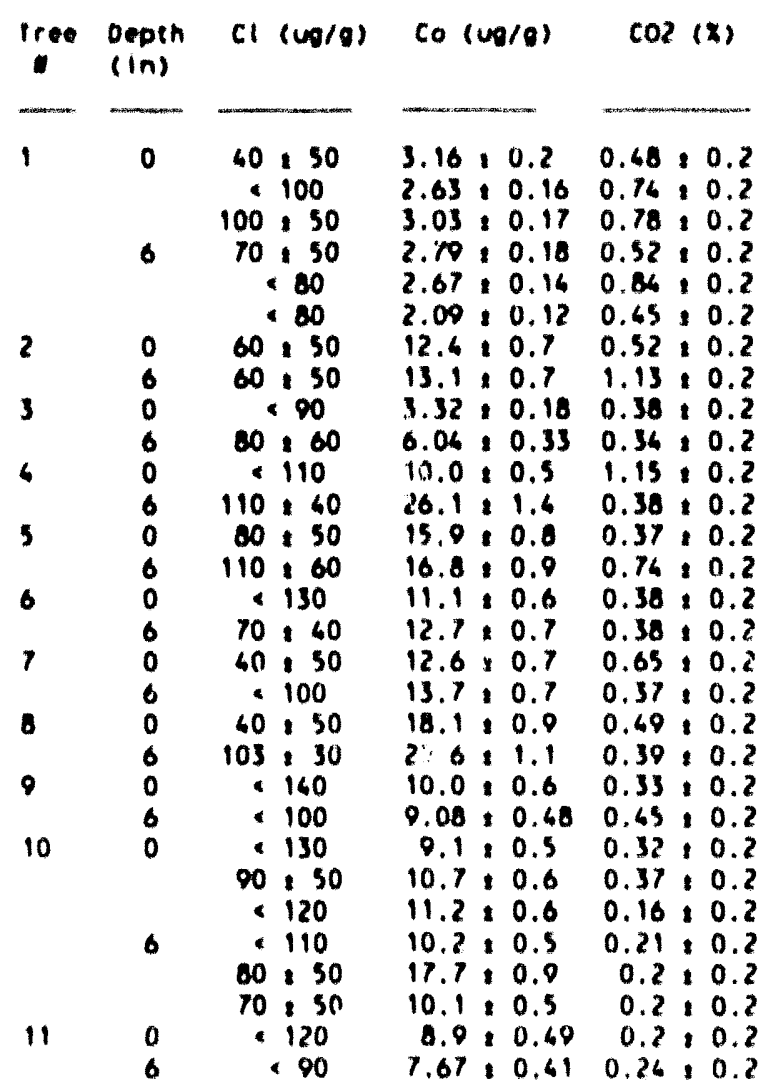

\section{$\operatorname{Cr}(0 q / 9)$}

$18.6: 1 . ?$ $16.3: 1.1$ $15.0 \div 1.1$ $17.1 \cdot 1.1$ $17.6: 1.1$ $22.2 \cdot 1.6$

$7.2: 0.8$ $11.2: 0.4$ $16.3: 1.1$ $22.7,1.5$ $18.0: 1.3$ $28.9,1.9$ $26.1,1.6$ $51.7: 3.2$ $16.6,1.3$ $28.8+1.9$ $16.8: 1.1$ $16.1,1.1$ $27.6 \times 1.9$ $31.3: 2.0$ $23.6: 1.6$ $25.1: 1.6$ $15.6,1.1$ $13.9: 1.1$ $19.3 \div 1.4$ $26.3: 1.6$ $31.8: 2.1$ $20.6: 1.6$ $27.1,1.8$ $20.9,1.9$
C. $(u g / \theta)$ Cu $(\omega g / \theta)$ or $(\omega p / \theta)$

Eu $(\omega 0 / 9)$

\begin{tabular}{|c|c|c|c|c|c|c|}
\hline $\begin{array}{l}3.22 \\
2.04 \\
2.60 \\
2.90 \\
3.11 \\
3.36 \\
5.21 \\
5.16 \\
2.79 \\
6.76 \\
4.10 \\
4.78 \\
7.66 \\
9.0 \\
7.33 \\
9.5 \\
4.94 \\
3.32 \\
3.05 \\
4.78 \\
4.20 \\
4.12 \\
3.28 \\
2.90 \\
4.12 \\
4.99 \\
6.82 \\
3.62 \\
4.62 \\
4.93\end{array}$ & $\begin{array}{l}10.21 \\
10.13 \\
0.19 \\
0.18 \\
0.20 \\
0.19 \\
0.3 \\
0.29 \\
0.16 \\
0.29 \\
0.26 \\
0.28 \\
10.64 \\
0.5 \\
0.62 \\
10.6 \\
10.33 \\
0.30 \\
0.30 \\
10.28 \\
10.25 \\
10.3 \\
10.20 \\
10.18 \\
10.25 \\
10.32 \\
10.40 \\
10.22 \\
10.30 \\
10.29\end{array}$ & 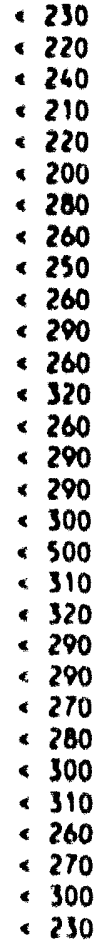 & $\begin{array}{r}11.3 \\
20.1 \\
10.6 \\
8.3 \\
8.8 \\
9.5 \\
10.5 \\
5.8 \\
11.6 \\
10.6 \\
7.7 \\
7.2 \\
6.5 \\
7.0 \\
6.3 \\
7.0 \\
6.8 \\
5.4 \\
6.3 \\
6.5 \\
7.3 \\
6.9 \\
12.8 \\
13.9 \\
10.6 \\
11.8 \\
7.8 \\
10.2 \\
7.9 \\
6.2\end{array}$ & $\begin{array}{l}0.9 \\
1.7 \\
0.8 \\
0.7 \\
0.7 \\
0.8 \\
0.9 \\
0.5 \\
0.9 \\
1.0 \\
0.7 \\
0.6 \\
0.6 \\
0.7 \\
0.6 \\
0.7 \\
0.0 \\
0.5 \\
0.6 \\
0.7 \\
0.7 \\
0.6 \\
1.1 \\
1.2 \\
0.9 \\
0.9 \\
0.7 \\
1.0 \\
1.7 \\
10.6\end{array}$ & $\begin{array}{l}1.28 \\
1.50 \\
1.19 \\
1.07 \\
1.06 \\
1.07 \\
1.69 \\
1.48 \\
1.15 \\
1.10 \\
1.03 \\
1.21 \\
1.45 \\
1.32 \\
1.50 \\
1.32 \\
1.80 \\
1.57 \\
1.78 \\
1.82 \\
1.27 \\
1.28 \\
1.36 \\
1.40 \\
1.31 \\
1.26 \\
1.30 \\
1.26 \\
1.27 \\
0.97\end{array}$ & $\begin{array}{l}0.08 \\
0.09 \\
0.08 \\
0.06 \\
0.07 \\
0.05 \\
0.09 \\
0.09 \\
0.07 \\
0.07 \\
0.07 \\
0.08 \\
0.09 \\
0.07 \\
0.08 \\
0.07 \\
0.12 \\
0.07 \\
0.09 \\
0.09 \\
0.09 \\
0.08 \\
0.08 \\
0.07 \\
0.09 \\
0.07 \\
0.08 \\
0.08 \\
0.06 \\
0.06\end{array}$ \\
\hline
\end{tabular}




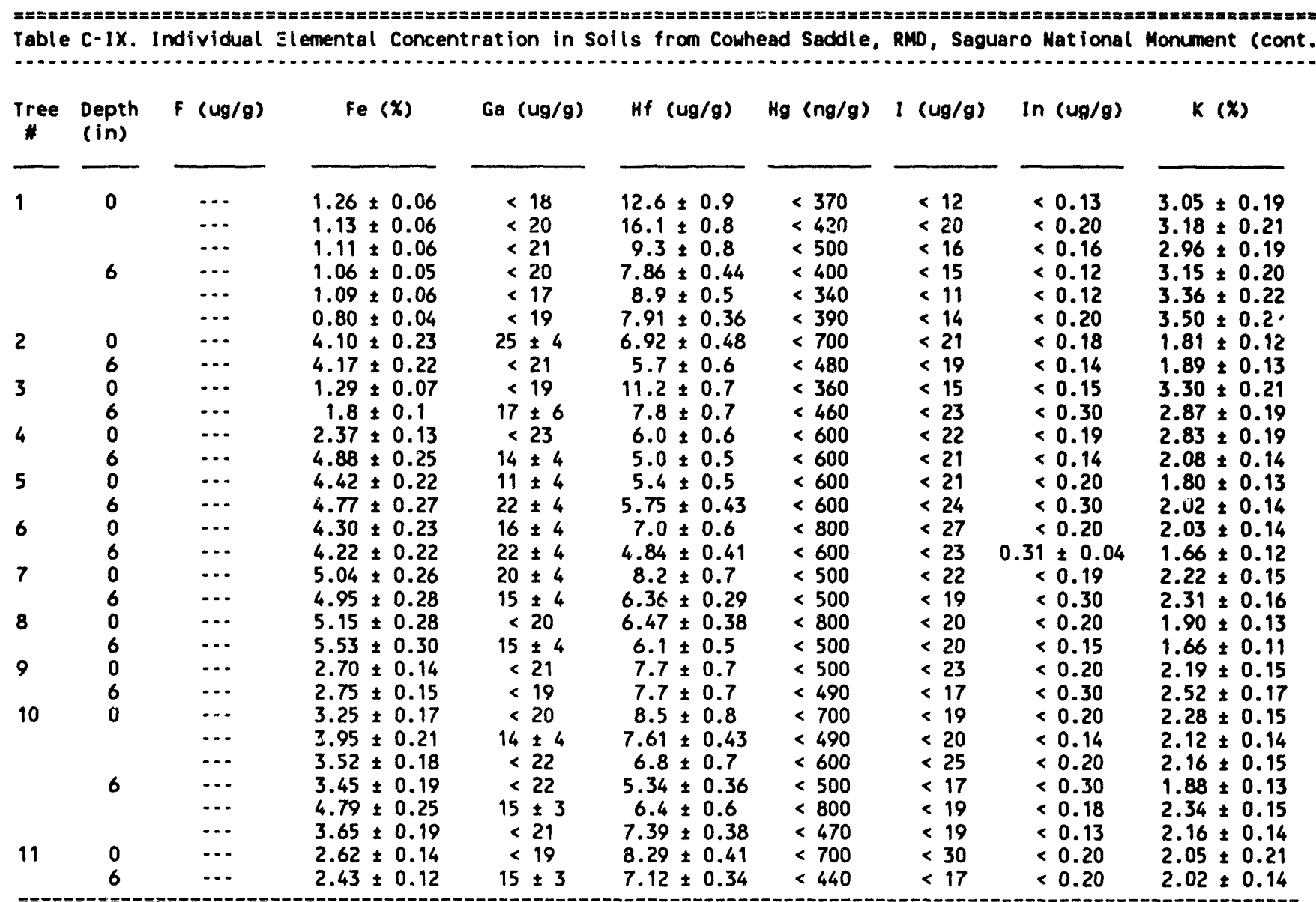

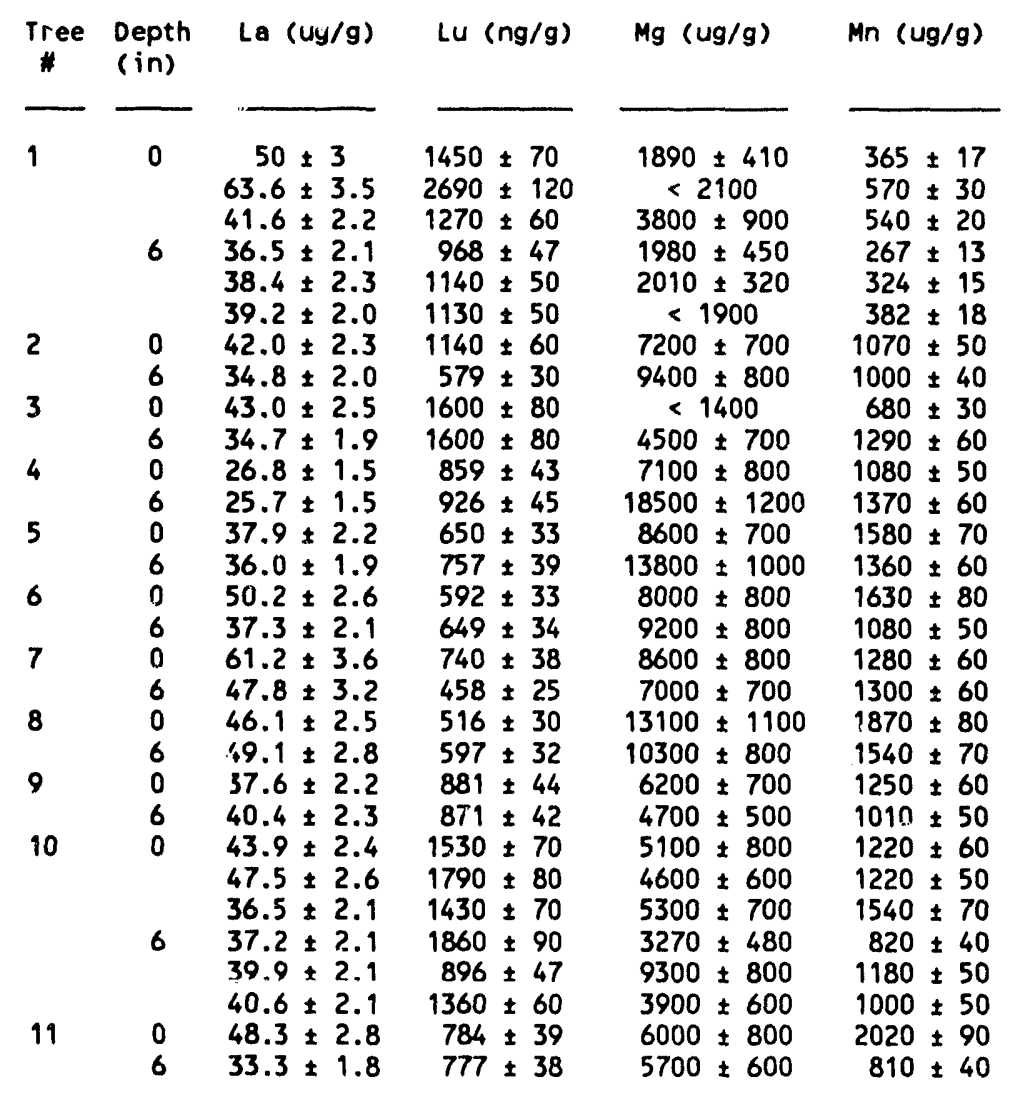

$\mathrm{Na}(\%)$ Nd (ug/g) pH (units)
$\mathrm{Rb}(u g / g)$

\begin{tabular}{|c|c|c|c|c|}
\hline $\begin{array}{l}1.98 \\
2.15 \\
1.95 \\
1.92 \\
1.92 \\
2.22 \\
2.27 \\
2.32 \\
2.03 \\
2.07 \\
2.11 \\
1.81 \\
1.88 \\
1.71 \\
2.1 \\
1.88 \\
2.23 \\
2.27 \\
2.11 \\
2.16 \\
1.95 \\
2.15 \\
2.39 \\
2.33 \\
1.98 \\
2.84 \\
1.95 \\
2.25 \\
1.84 \\
1.73\end{array}$ & $\begin{array}{ll} \pm & 0.08 \\
\pm & 0.10 \\
\pm & 0.08 \\
\pm & 0.08 \\
\pm & 0.08 \\
\pm & 0.10 \\
\pm & 0.08 \\
\pm & 0.10 \\
\pm & 0.09 \\
\pm & 0.09 \\
\pm & 0.09 \\
\pm & 0.08 \\
\pm & 0.08 \\
\pm & 0.08 \\
\pm & 0.1 \\
\pm & 0.08 \\
\pm & 0.09 \\
\pm & 0.10 \\
\pm & 0.10 \\
\pm & 0.09 \\
\pm & 0.08 \\
\pm & 0.09 \\
\pm & 0.11 \\
\pm & 0.10 \\
\pm & 0.08 \\
\pm & 0.12 \\
\pm & 0.07 \\
\pm & 0.09 \\
\pm & 0.08 \\
\pm & 0.07\end{array}$ & $\begin{array}{l}59 \pm 6 \\
85 \pm 9 \\
37 \pm 6 \\
35 \pm 5 \\
41 \pm 5 \\
42 \pm 5 \\
40 \pm 7 \\
34 \pm 6 \\
46 \pm 5 \\
49 \pm 7 \\
26 \pm 7 \\
27 \pm 5 \\
37 \pm 6 \\
37 \pm 9 \\
47 \pm 8 \\
37 \pm 6 \\
66 \pm 7 \\
56 \pm 7 \\
56 \pm 8 \\
43 \pm 6 \\
40 \pm 6 \\
45 \pm 7 \\
43 \pm 7 \\
42 \pm 5 \\
42 \pm 7 \\
46 \pm 7 \\
47 \pm 8 \\
36 \pm 5 \\
54 \pm 8 \\
27 \pm 4\end{array}$ & 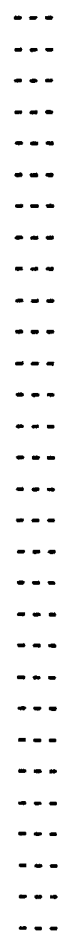 & $\begin{array}{r}130 \pm 9 \\
105 \pm 5 \\
150 \pm 7 \\
136 \pm 7 \\
135 \pm 6 \\
132 \pm 6 \\
99 \pm 6 \\
120 \pm 6 \\
138 \pm 7 \\
131 \pm 6 \\
120 \pm 6 \\
132 \pm 7 \\
142 \pm 7 \\
148 \pm 7 \\
146 \pm 8 \\
152 \pm 8 \\
132 \pm 7 \\
128 \pm 7 \\
111 \pm 6 \\
113 \pm 6 \\
133 \pm 7 \\
121 \pm 6 \\
114 \pm 6 \\
119 \pm 6 \\
117 \pm 6 \\
127 \pm 6 \\
190 \pm 9 \\
134 \pm 7 \\
118 \pm 6 \\
130 \pm 7\end{array}$ \\
\hline
\end{tabular}




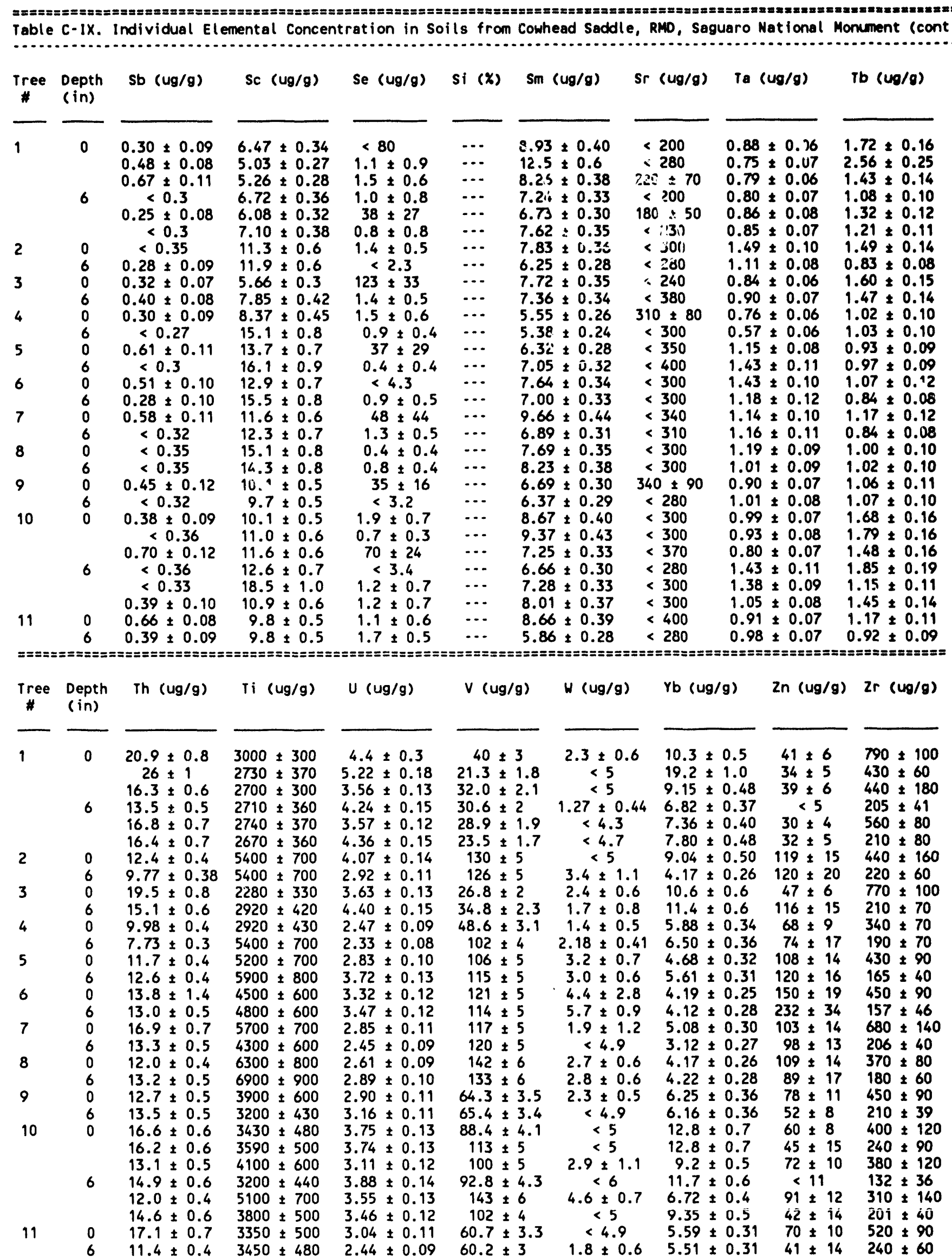




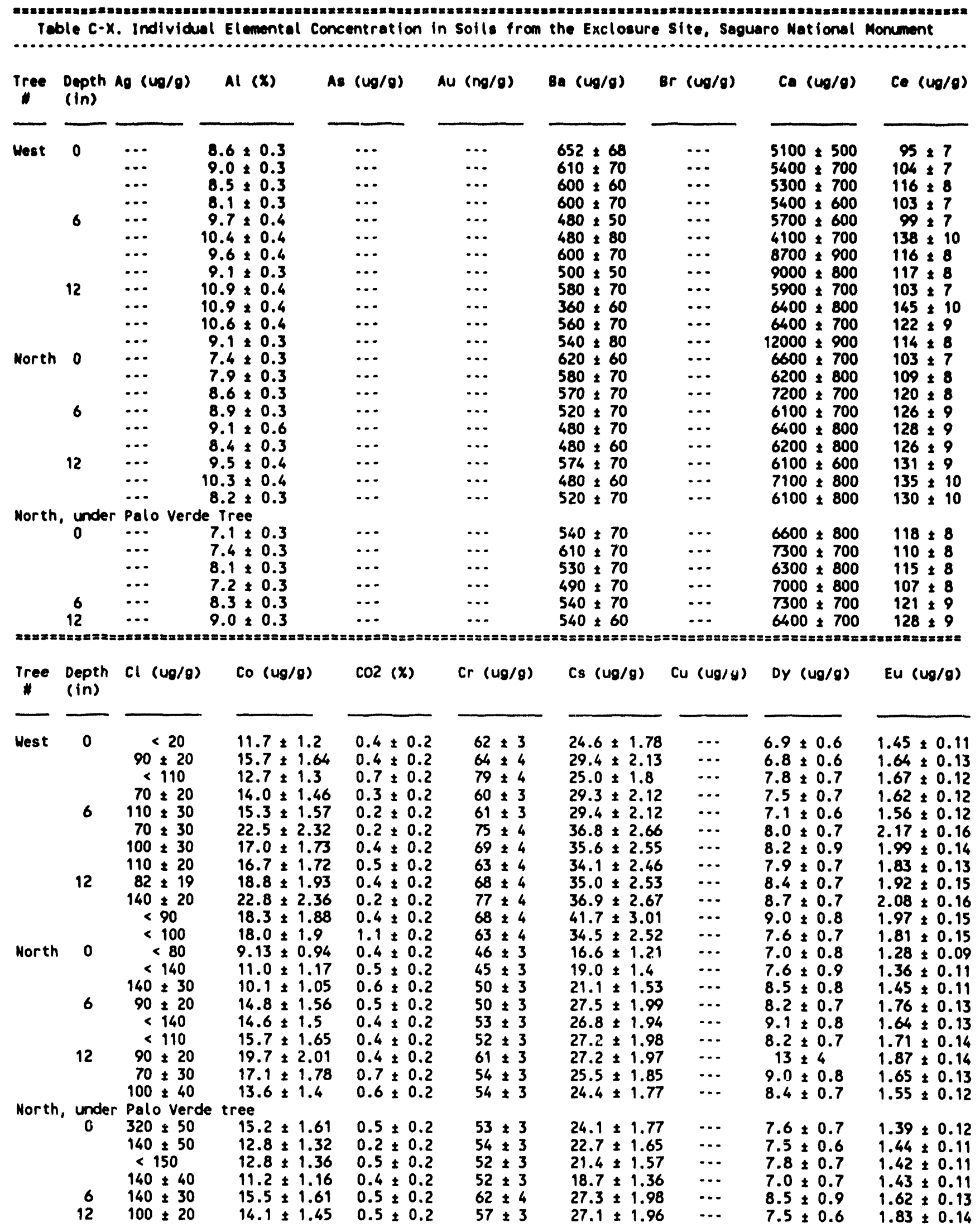




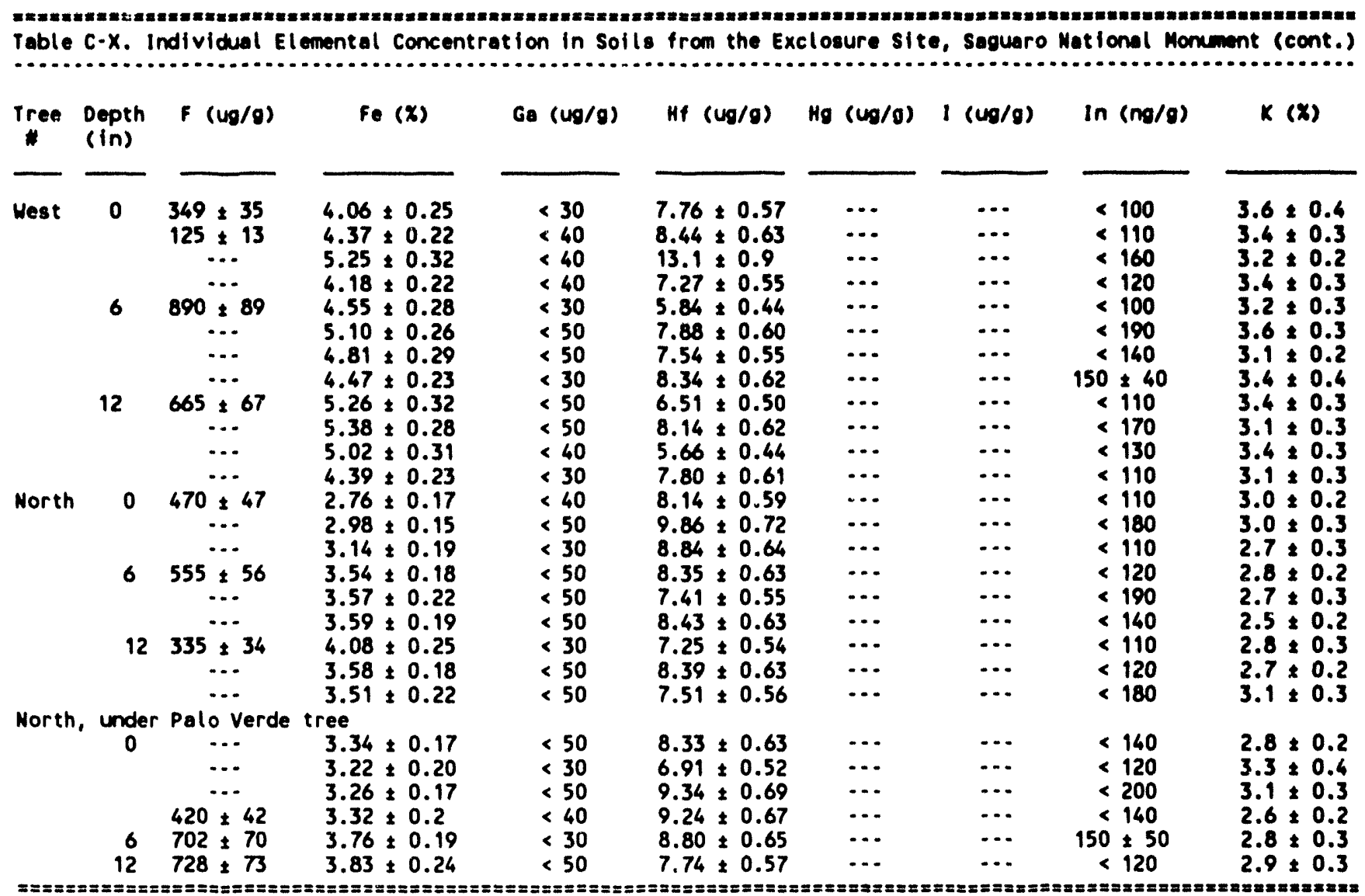

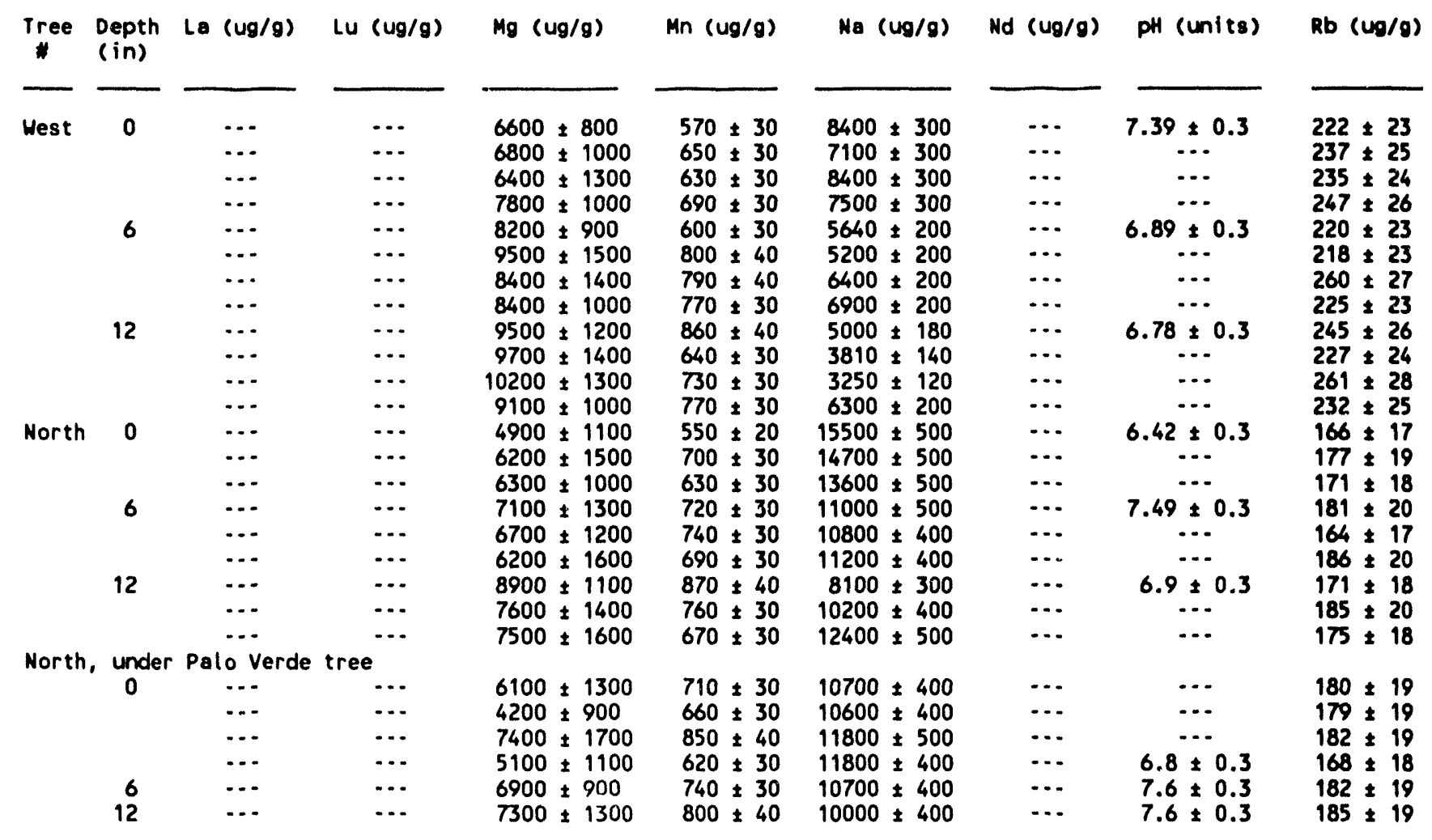




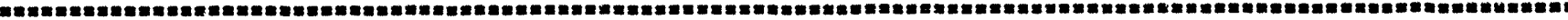
Table C-X. Individual Elemental Concentration in Solls from the Exclosure site, Saguaro Mational Monument (cont.)

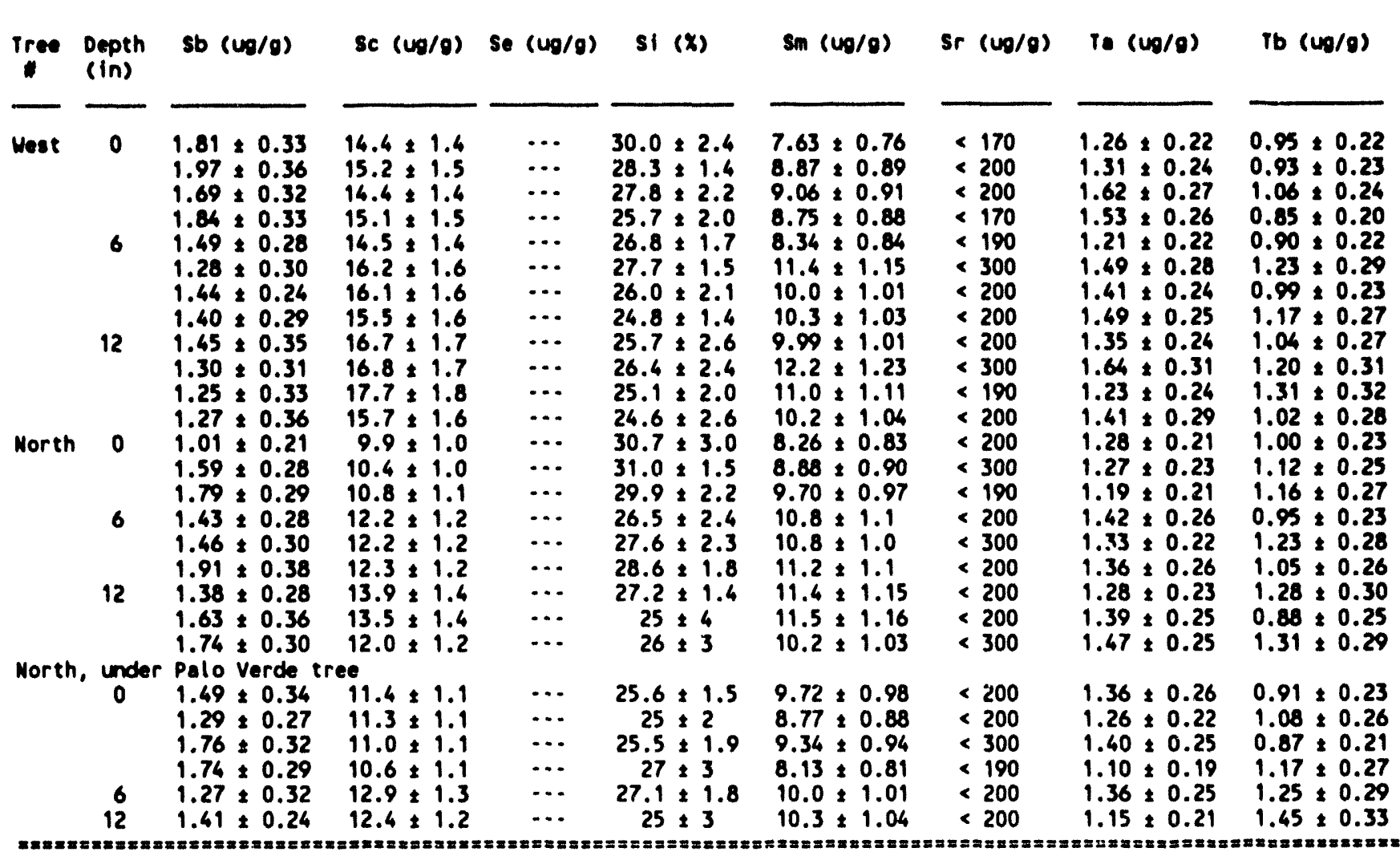

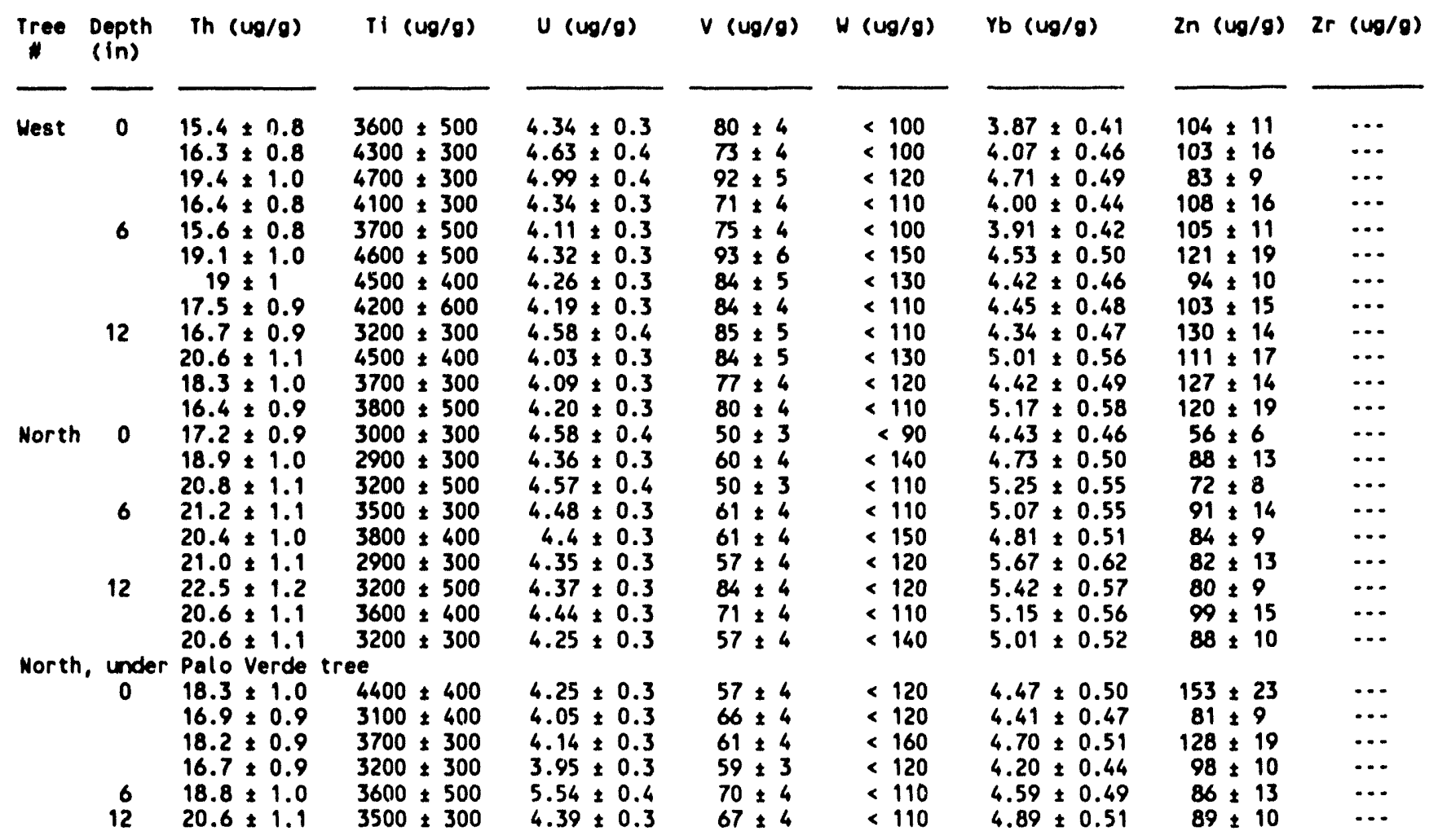




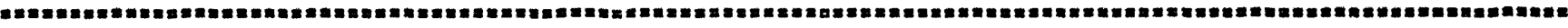
Table C-XI. Individual Elemental Concentration In Solls under Melen's Dome White Pinas, Saguaro Nationil Monument

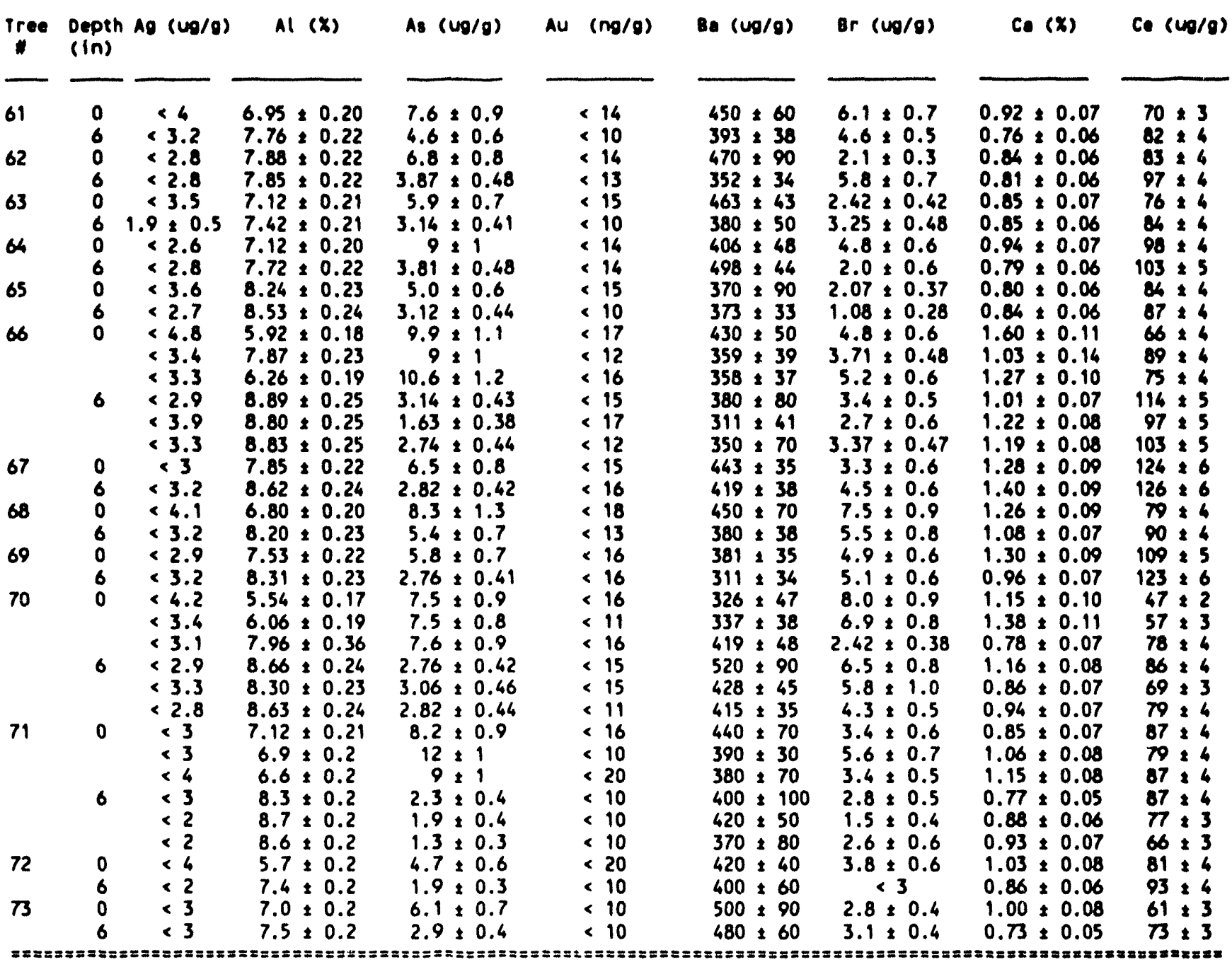

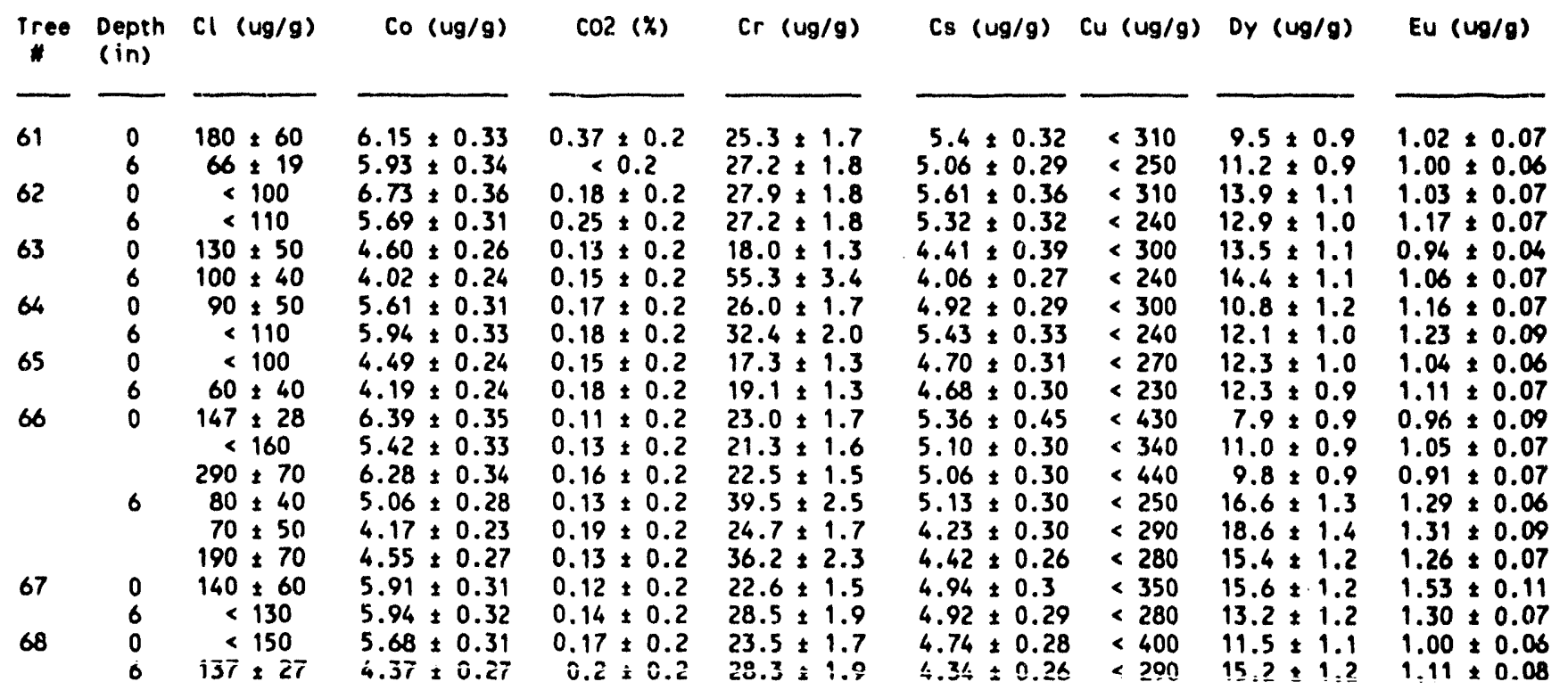


ioble C-Xl. Individual Elemental Concentration In Solls under Helen's Dome White Pines,

Soguaro National Monument (cont.)

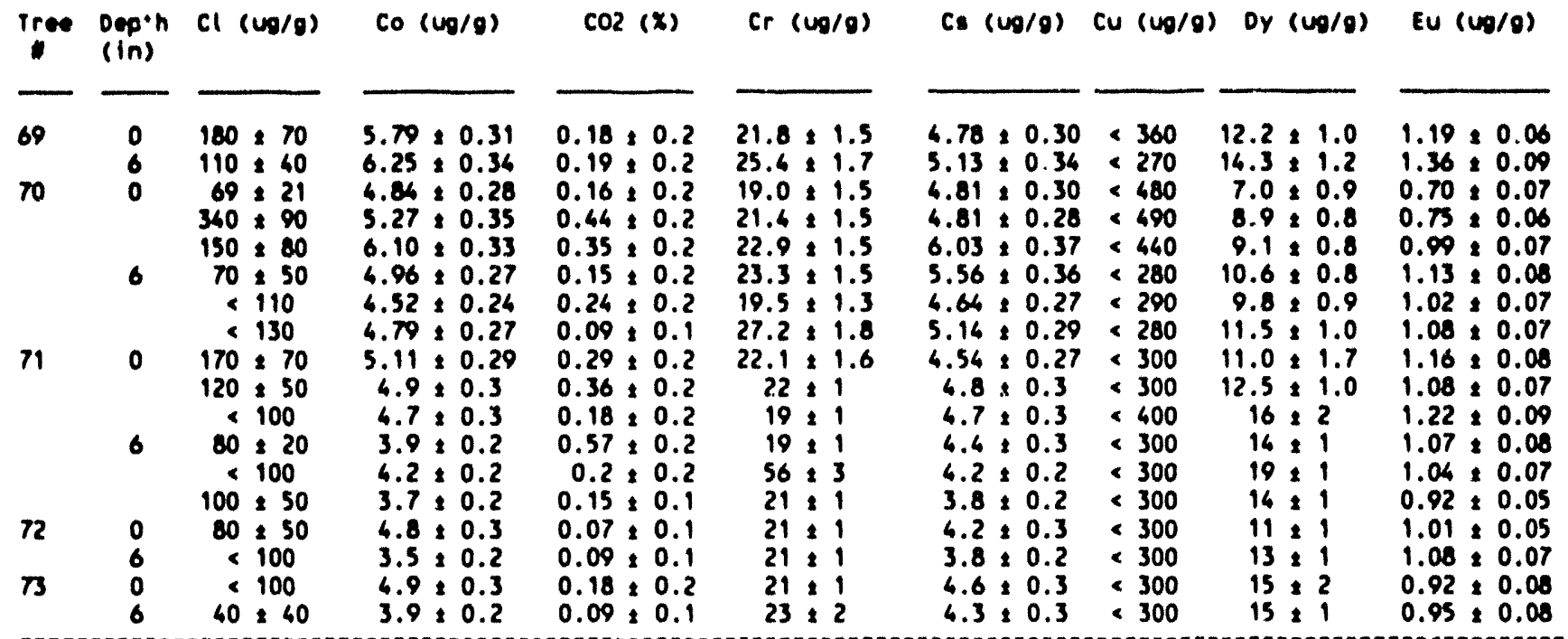

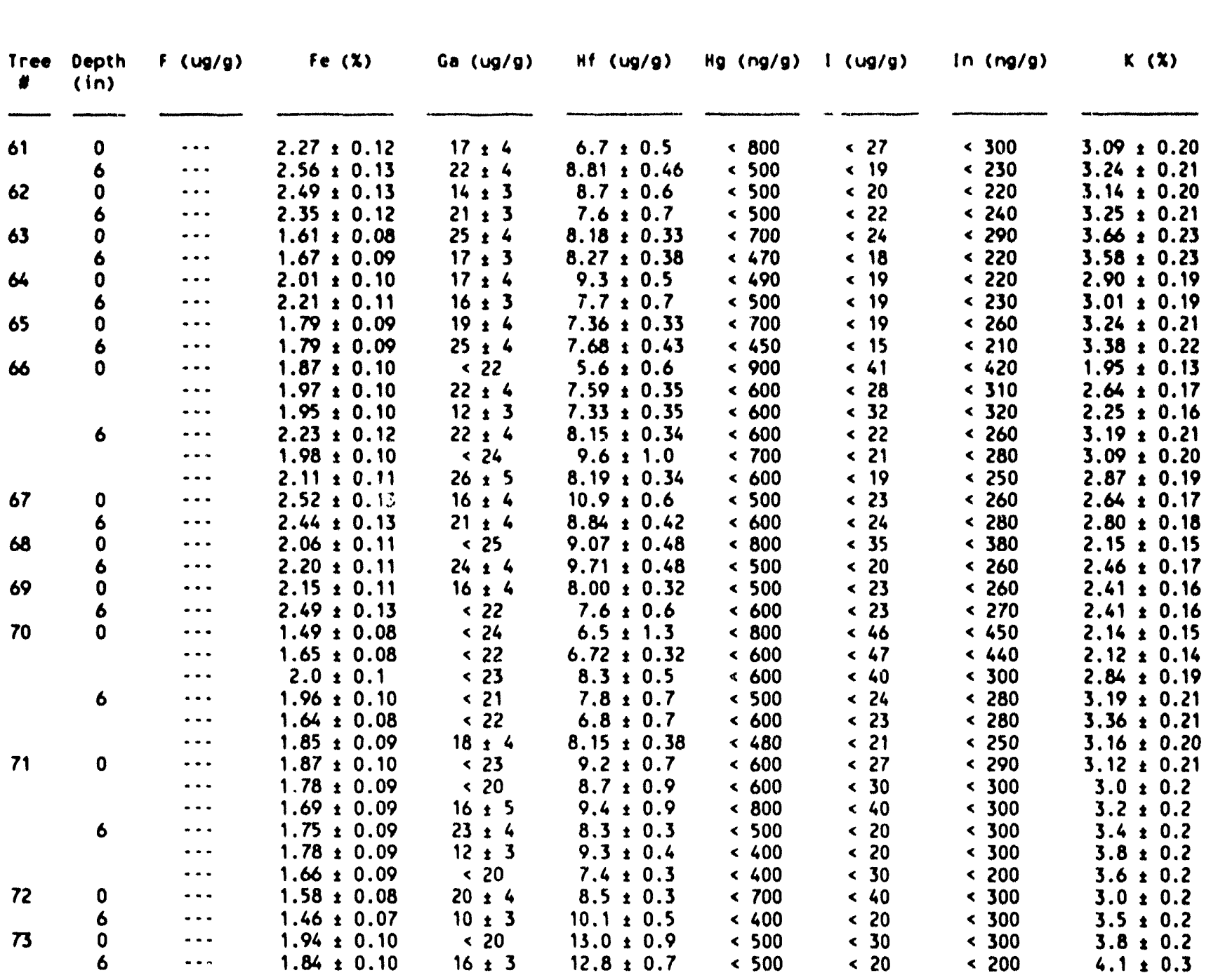




\begin{tabular}{|c|c|c|c|c|c|c|c|c|c|c|}
\hline Tree & $\begin{array}{l}\text { Depth } \\
\text { (In) }\end{array}$ & lo $(00 / 0)$ & lu $(n g / 0)$ & $H_{g}(u g / g)$ & $\operatorname{Mn}(\log / \theta)$ & Ne? & $(x)$ & Nd $(u g / g)$ & pH (unites) & $10(\omega p / g)$ \\
\hline 61 & $\begin{array}{l}0 \\
6 \\
0 \\
6 \\
0 \\
6 \\
0 \\
6 \\
0 \\
6 \\
0\end{array}$ & $\begin{array}{r}29.9: 1.6 \\
36.3: 2.1 \\
35.5: 2.2 \\
40.8: 2.2 \\
31.5: 1.8 \\
38.4: 2.2 \\
46.3: 2.7 \\
46.3: 2.3 \\
38.5: 2.1 \\
38.8: 2.2 \\
32.5: 1.7 \\
38.9: 2.1 \\
32.7: 1.8 \\
47.3: 2.5 \\
43.6: 2.4 \\
47.6: 2.7 \\
53.5: 3.2 \\
50.8: 2.8 \\
37.6: 2.0 \\
43.0: 2.2 \\
48.6: 2.9 \\
50.5: 2.7 \\
23.6: 1.3 \\
25.6: 1.5 \\
32.5: 1.8 \\
36: 2 \\
32.8: 1.8 \\
37.3: 2.1 \\
36.6: 2.1 \\
36: 2 \\
40: 2 \\
39: 2 \\
36: 2 \\
30: 2 \\
39: 2 \\
40: 2 \\
28: 2 \\
34: 2 \\
36\end{array}$ & 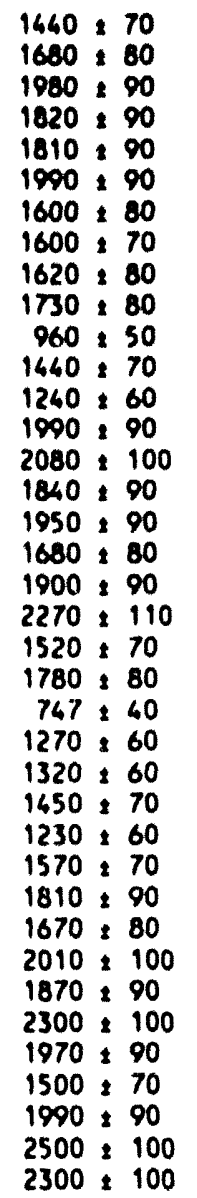 & 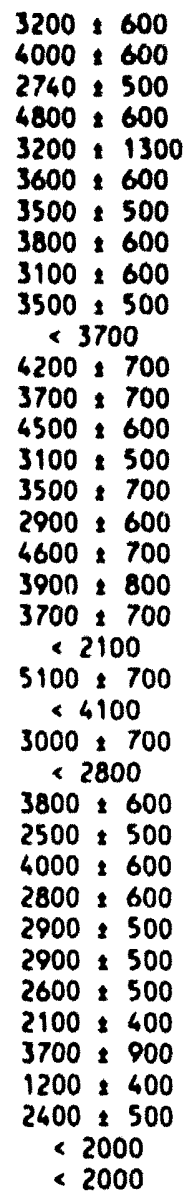 & $\begin{array}{r:}1360: 60 \\
886: 35 \\
1250: 60 \\
1110: 50 \\
1280: 50 \\
680: 30 \\
1000: 50 \\
580: 30 \\
740: 30 \\
640: 30 \\
2390: 110 \\
1710: 80 \\
2330: 100 \\
1640: 70 \\
860: 40 \\
610: 30 \\
1650: 70 \\
1120: 50 \\
2040: 90 \\
780: 40 \\
1590: 70 \\
790: 40 \\
3140: 140 \\
3770: 170 \\
2440: 110 \\
1140: 50 \\
1210: 50 \\
1170: 50 \\
1640: 70 \\
1300: 60 \\
1140: 50 \\
670: 30 \\
930: 40 \\
1160: 50 \\
1530: 70 \\
700: 30 \\
1760: 80 \\
770: 30\end{array}$ & $\begin{array}{l}1.37 \\
1.64 \\
1.61 \\
1.60 \\
1.76 \\
1.83 \\
1.70 \\
1.90 \\
1.99 \\
2.11 \\
1.29 \\
2.02 \\
1.52 \\
2.30 \\
2.44 \\
2.42 \\
2.12 \\
2.33 \\
2.02 \\
2.33 \\
2.10 \\
2.27 \\
1.28 \\
1.43 \\
1.99 \\
2.23 \\
2.08 \\
2.26 \\
1.94 \\
1.85 \\
2.15 \\
2.28 \\
2.32 \\
2.37 \\
1.98 \\
2.26 \\
1.40 \\
1.54\end{array}$ & 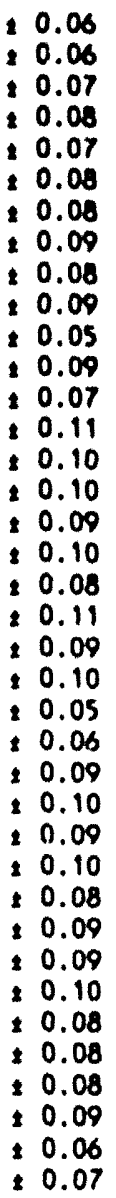 & $\begin{array}{l:l}37 & : \\
35 & 6 \\
36 & 6 \\
73 & 9 \\
38 & 7 \\
32 & 5 \\
50 & 7 \\
63 & 8 \\
39 & 7 \\
30 & 5 \\
37 & 9 \\
38 & 7 \\
40 & 7 \\
60 & 9 \\
53 & 8 \\
46 & 6 \\
64 & 8 \\
72 & 9 \\
42 & 8 \\
47 & 6 \\
65 & 8 \\
66 & 8 \\
25 & 8 \\
21 & 6 \\
33 & 8 \\
40 & 7 \\
32 & 6 \\
26 & 6 \\
41 & 7 \\
35 & 5 \\
42 & 6 \\
31 & 4 \\
36 & 4 \\
21 & 3 \\
24 & 4 \\
35 & 4 \\
25 & 5 \\
26 & 3\end{array}$ & 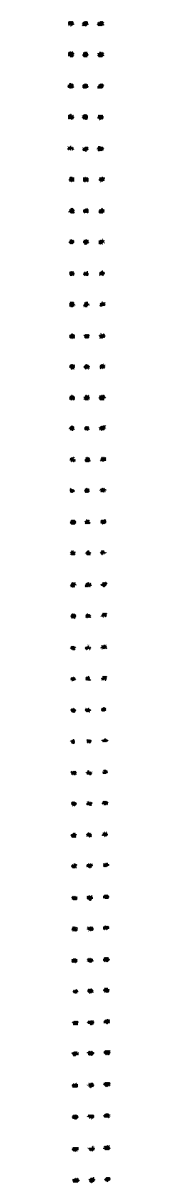 & 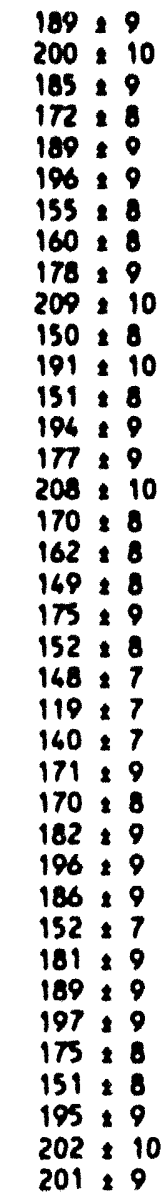 \\
\hline
\end{tabular}

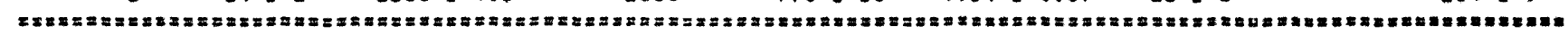

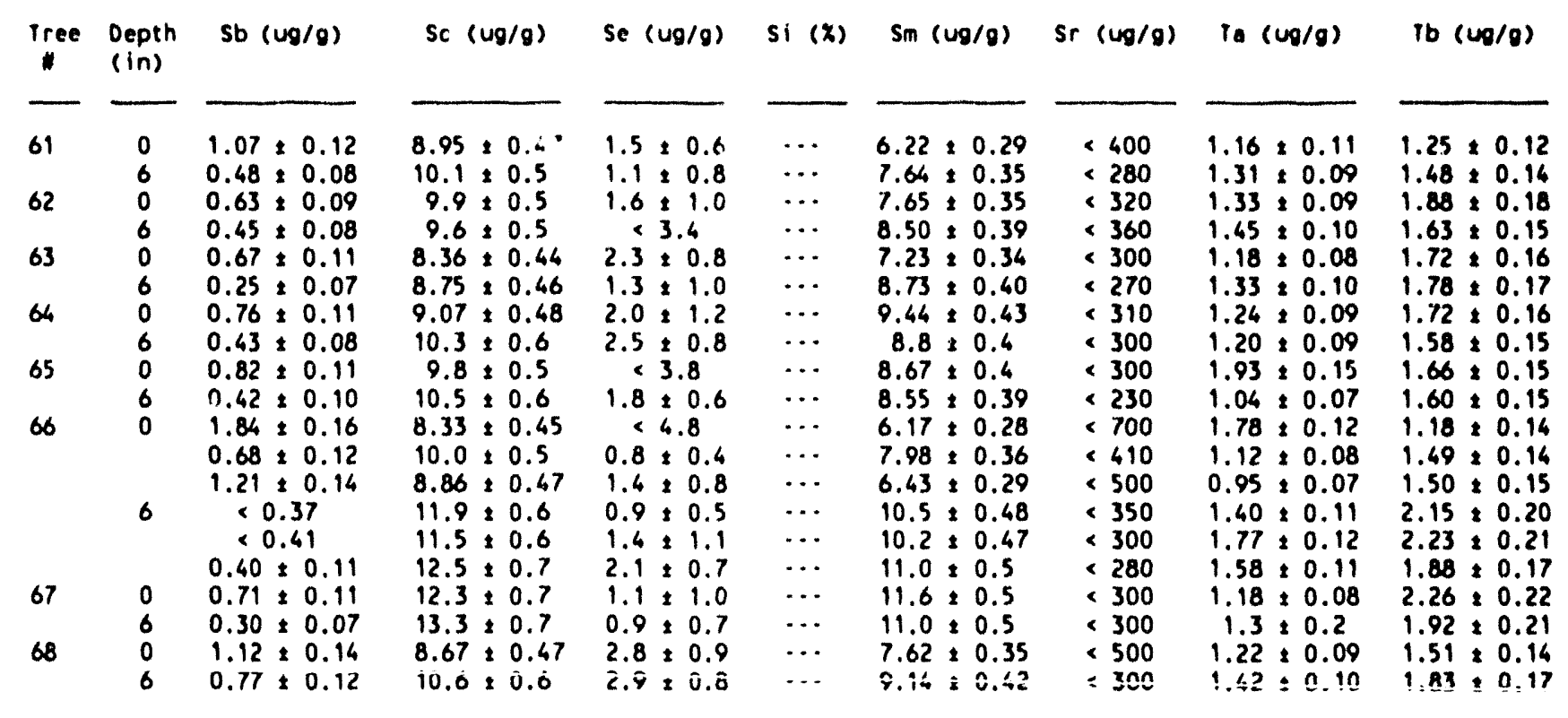




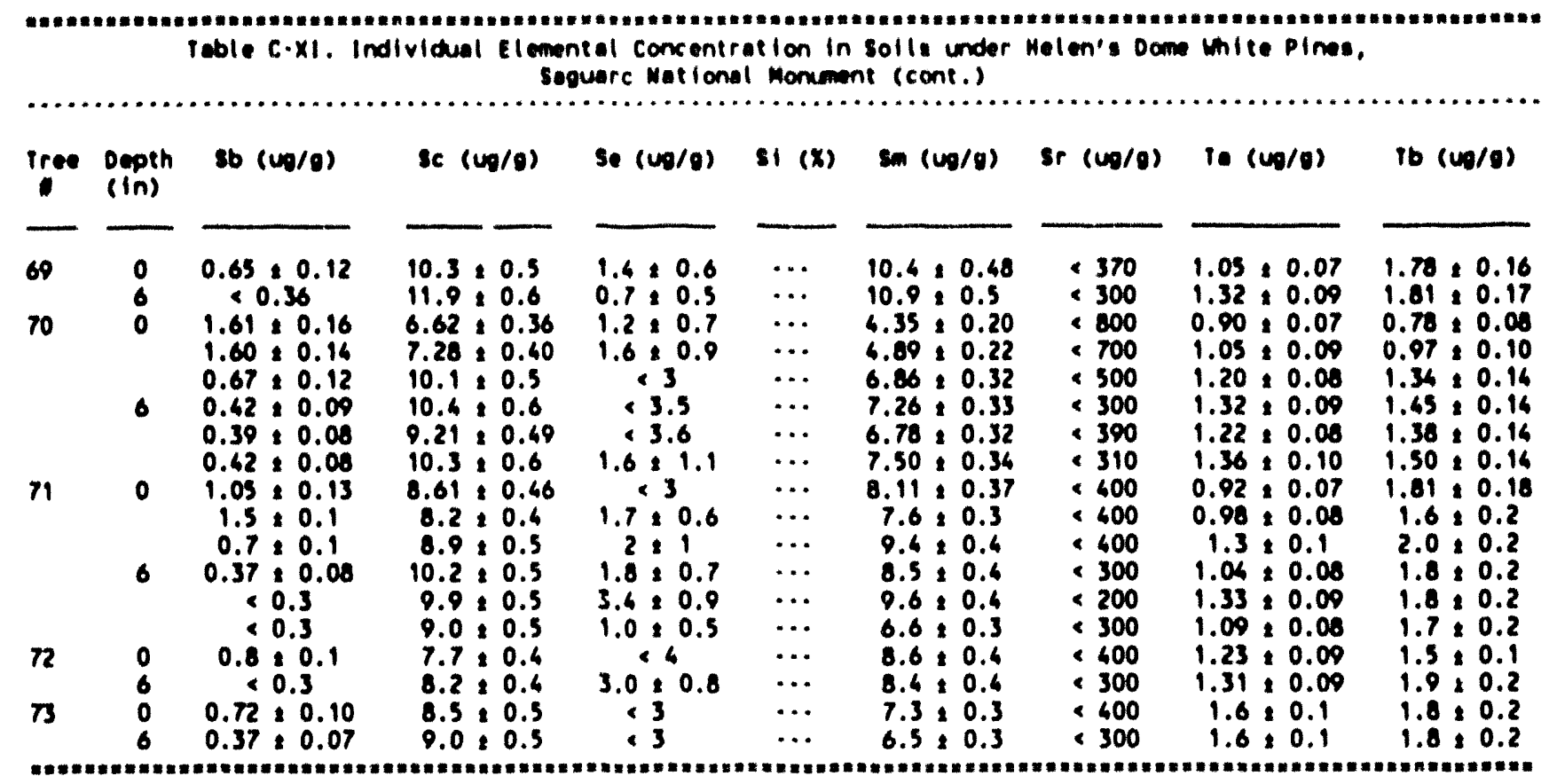

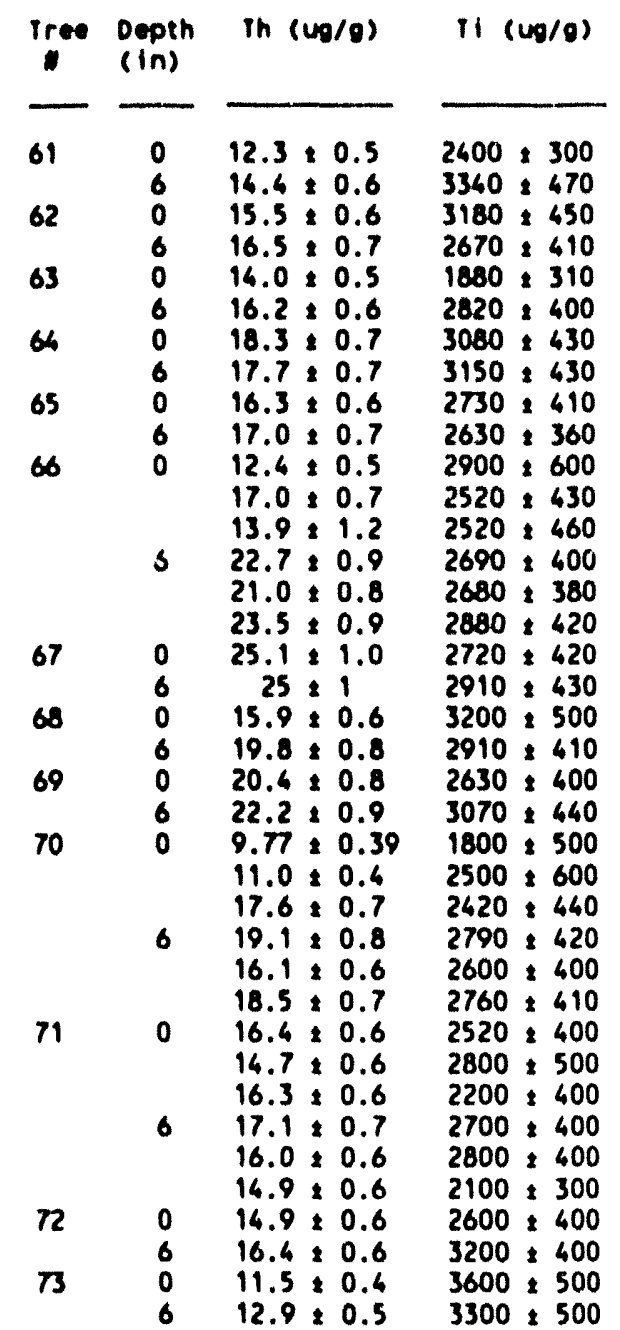

$U(u g / g)$

$2.85 \div 0.11$

$3.78 \div 0.14$

$4.71 \div 0.17$

$4.97 \div 0.17$

$3.40=0.13$

$4.96=0.17$

$4.83 \div 0.17$

$3.72 \div 0.13$

$5.88=0.21$

$3.88 \div 0.14$

$4.98 \div 0.18$

$3.30 * 0.12$

$3.03 \div 0.12$

$4.74 \div 0.17$

$4.90 \div 0.17$

$4.66 \div 0.17$

$6.30: 0.15$

$4.34 \div 0.16$

$3.51: 0.13$

$4.33 \div 0.16$

$4.23=0.15$

$4.37 \div 0.16$

$2.95: 0.12$

$3.49 \neq 0.13$

$3.53 \div 0.13$

$3.70 \div 0.13$

$3.44 \div 0.12$

$3.87 \div 0.14$

$3.17 \div 0.12$

$3.4 \div 0.1$

$4.8 \div 0.2$

$4.2 \div 0.1$

$4.8 \div 0.2$

$3.6 \div 0.1$

$3.4 \div 0.1$

$4.6 \div 0.2$

$3.8 \div 0.1$

$v(00 / 9)$

$v(u g / g)$

Yo $(40 / 0)$

$\ln (40 / 9)$

$\operatorname{2r}(00 / 9)$

$40.8: 3.0$

$48.7 \div 2.8$

$49.2 \times 3.2$

$42.0 \div 2.6$

$29.9 \div 2.6$

$27.9 \div 2.2$

$41.1 \div 2.8$

$39.4 \div 2.4$

$38.8 \div 2.7$

$33.2 \div 2.2$

$33.8 \div 3.8$

$32.9 \div 3.0$

$26.9 \div 3.4$

$30.9 \div 2.4$

$32.2 \div 2.5$

$\begin{array}{r:l}30.2 & 2.4 \\ 37 & 3\end{array}$

$36.2 \div 2.7$

$38.1 \div 3.6$

$40.5 \div 2.5$

$36.9+2.9$

$30.5=2.8$

$26.6=3.7$

$33.2 \times 4.7$

$34.0 \div 3.5$

$37.2 \div 2.7$

$26.9 \div 2.4$

$35.2 \times 2.9$

$38.3 \div 3.5$

34,3

$23 \div 2$

$29 \div 2$

$23+2$

$22 \div 2$

$27 \div 2$

$33 \div 2$

$36 \div 3$

$30+2$

< 4.8

$2.1 * 0.7$

C 4.7

$<4.5$

$<4.9$

$<4.7$

$<4.9$

$<4.9$

$<5$

$<4.8$

$2.1 \div 0.7$

$<6$
$<6$

$4.4 \div 2.8$

$<6$

$3.6 \div 1.6$

$<5$

$<6$

< 6

< 6

$<6$

$6.0 \div 2.8$

$1.4 \div 0.6$ $+6$

$3.0 \div 2.6$ $<5$ $<5$

$2.9 \div 0.8$

$<6$

$3+2$

$2.5 \div 1.0$

< 5

$<5$

$<5$

$<6$

$<5$

$<5$

$2.3 \div 0.7$
$0.5 \times 0.5$

$11.6 \div 0.6$

$13.2: 0.7$

$12.3: 0.6$

$12.1: 0.7$

$16.4: 0.7$

$11.0: 0.6$

$11.0: 0.6$

$11.4 \div 0.6$

$11.6 \div 0.6$

$7.18: 0.5$

$10.1+0.5$

$8.38: 0.47$

$16.6 \div 0.7$

$16.4 \div 0.8$

$14.0 \div 0.7$

$13.5: 0.7$

$11.9: 0.6$

$14.4: 0.7$

$16.1: 0.8$

$10.8: 0.6$

$12.3 \div 0.6$

$5.95: 0.35$ $9.6 \div 0.5$

$8.96 \div 0.69$ $10.1 \div 0.5$ $9.41: 0.5$

$12.0 \div 0.7$

$12.6: 0.7$

$12.1+0.6$

$14.7 \div 0.8$

$13.5 \div 0.7$

$16.4 \div 0.8$

$14.0 \div 0.7$

$10.3 \div 0.5$

$13.8 \div 0.7$

$17.3 \div 0.8$
$69 \div 10$

$32: 20$

$70: 10$

$52 \div 8$

$56: 8$

$25: 16$

$58 \div 0$

$56: 8$

$52: 7$

$28: 14$

$78 \div 11$

$72+24$

$106 \div 16$

$70 \div 10$

$55 \div 8$

$56 \div 19$

$76 \div 10$

$68: 10$

$83: 11$

$57 \div 20$

$87 \times 12$

$70 \div 10$

$82 \div 12$

$63+31$

$68 \div 9$

$<10$

$37 \div 6$

$68 \div 9$

$90 \div 12$

$80 \div 10$

$60 \div 9$

$30 \div 20$

$49 \div 7$

$44 \div 6$

$36 \div 6$

$20 \div 20$

$80 \div 10$

$<9$
340,80

$190: 45$

$490 \div 100$

$197 \div 41$

$340 \div 140$

$216: 46$

$610=150$

$230 \div 70$

$560 \div 110$

$200: 60$

$390 \div 100$

$212 \div 48$

$490 \div 110$

$160 \div 60$

$450: 90$

$209: 48$

$690: 110$

$280 \div 50$

$490=150$

$310: 60$

$530 \div 140$

$207 \div 42$

$250 \div 80$

$210=50$

$520 \div 100$

$213 \div 40$

$340 \div 70$

$520 \div 100$

$450 \div 90$

$260 \div 40$

$290 \div 50$

$260 \div 70$

$240 \div 40$

$210 \div 30$

$310 \div 50$

$390 \div 70$

$330 \div 50$

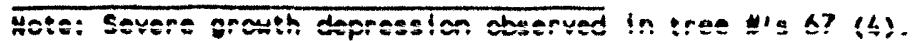




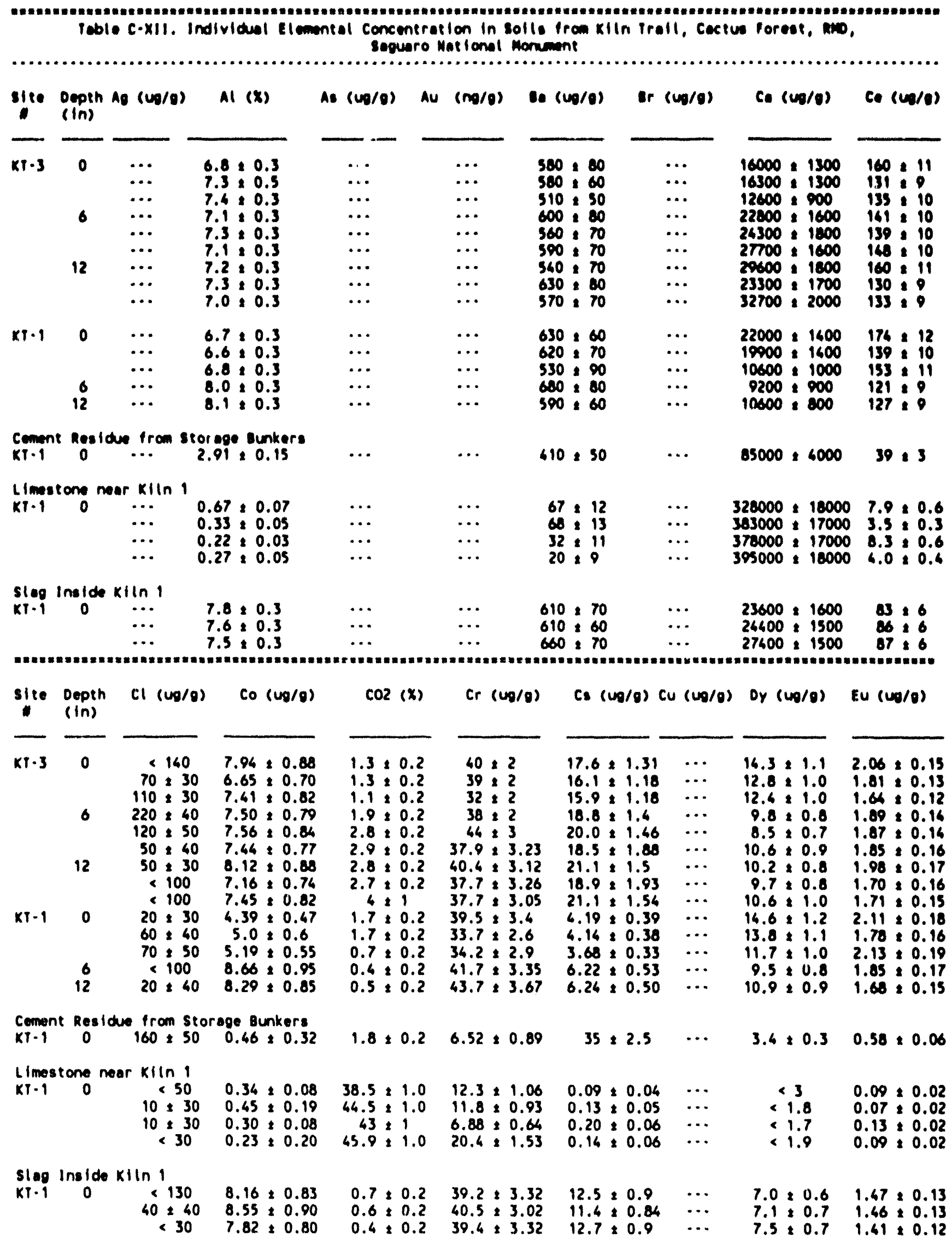




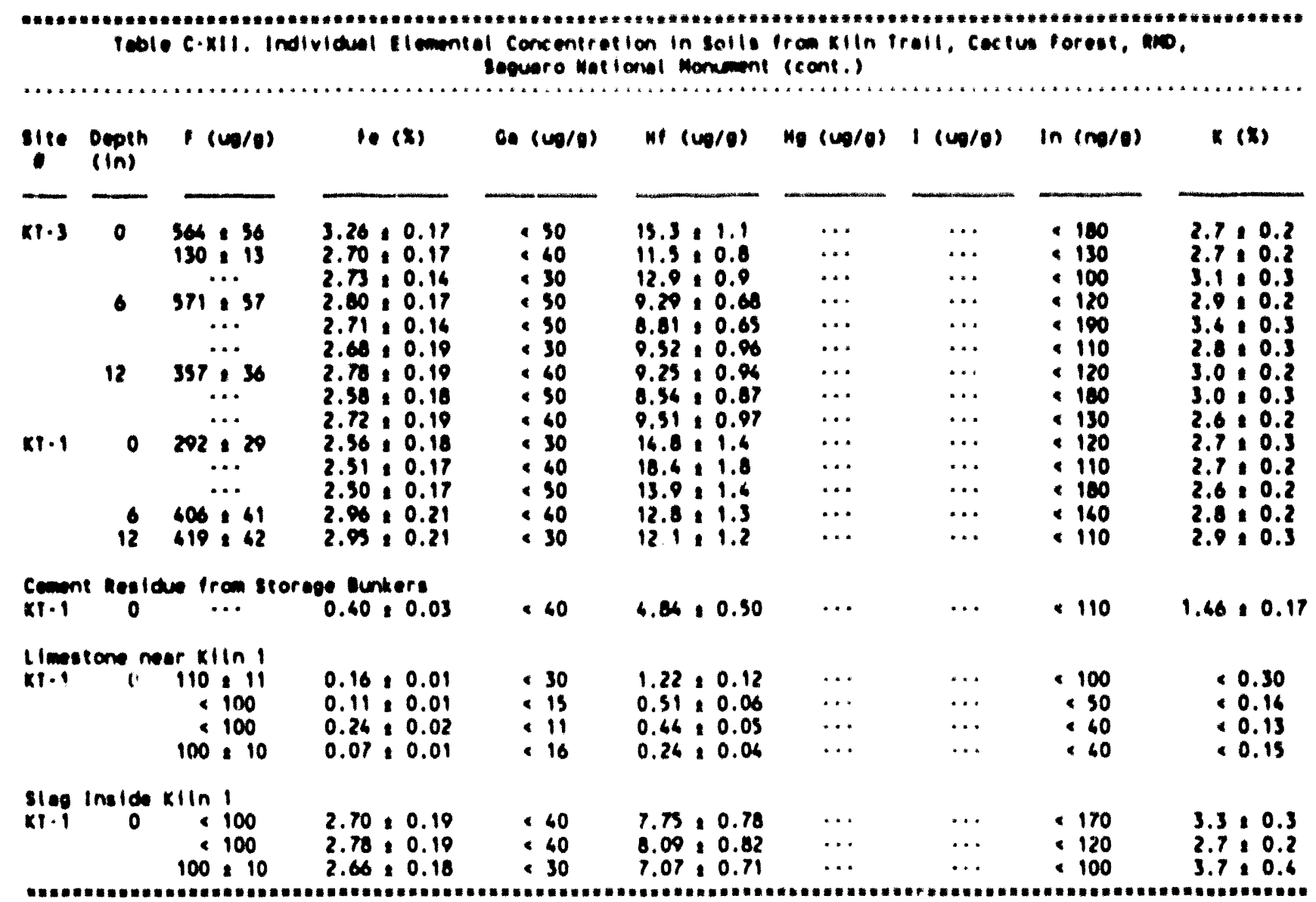

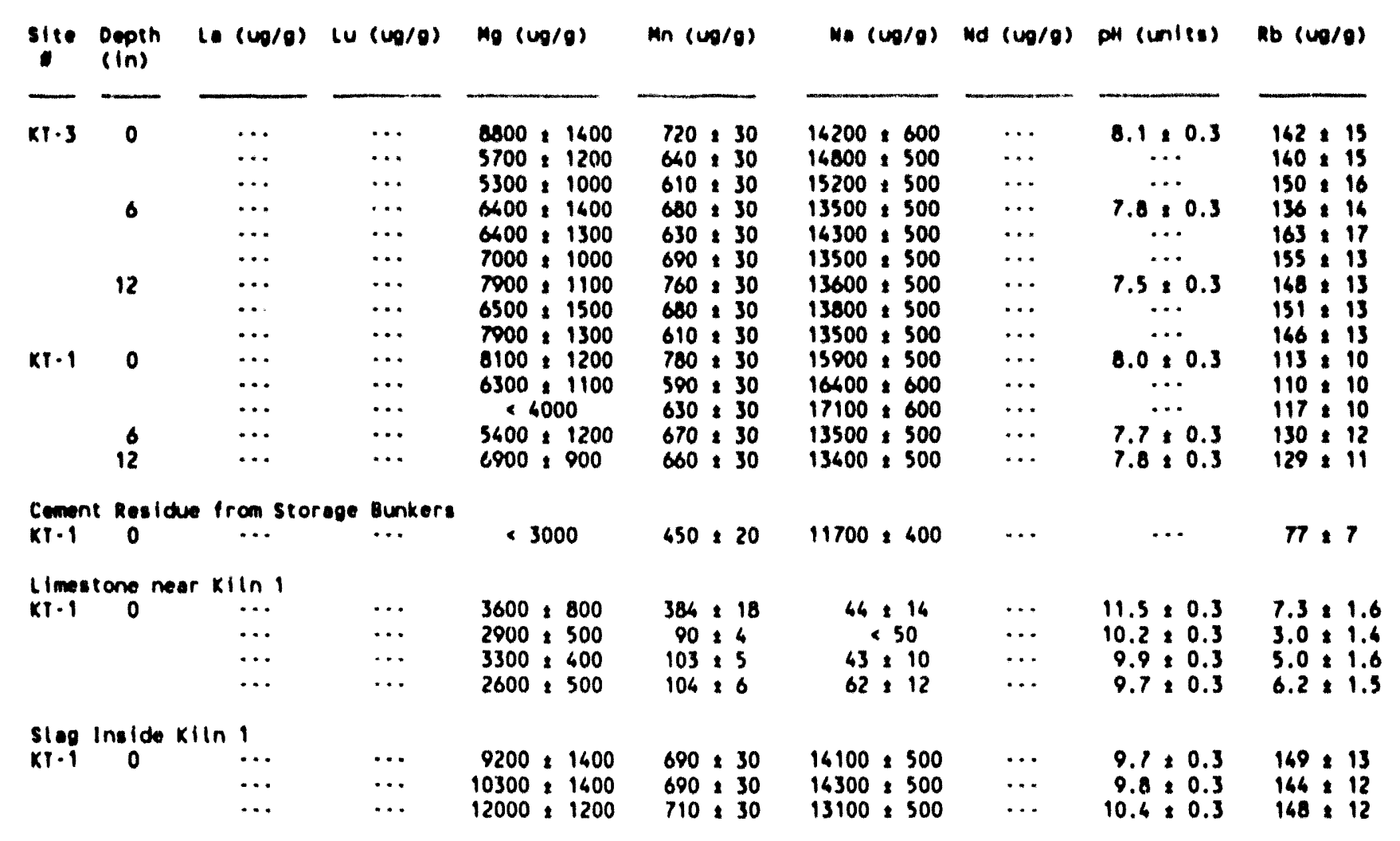




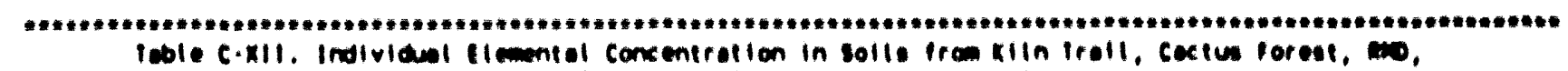
secuero vationat moniment (cont.)

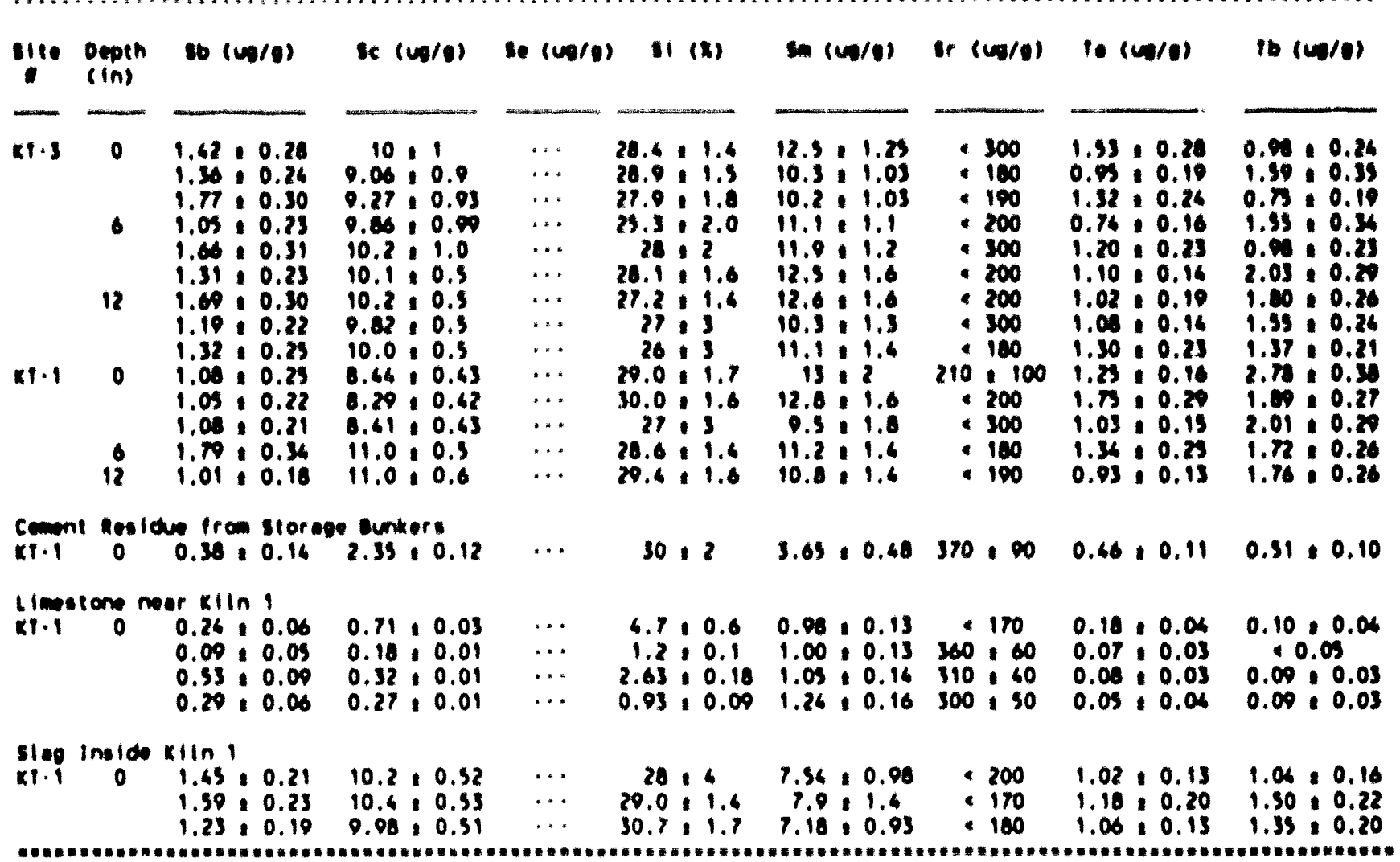

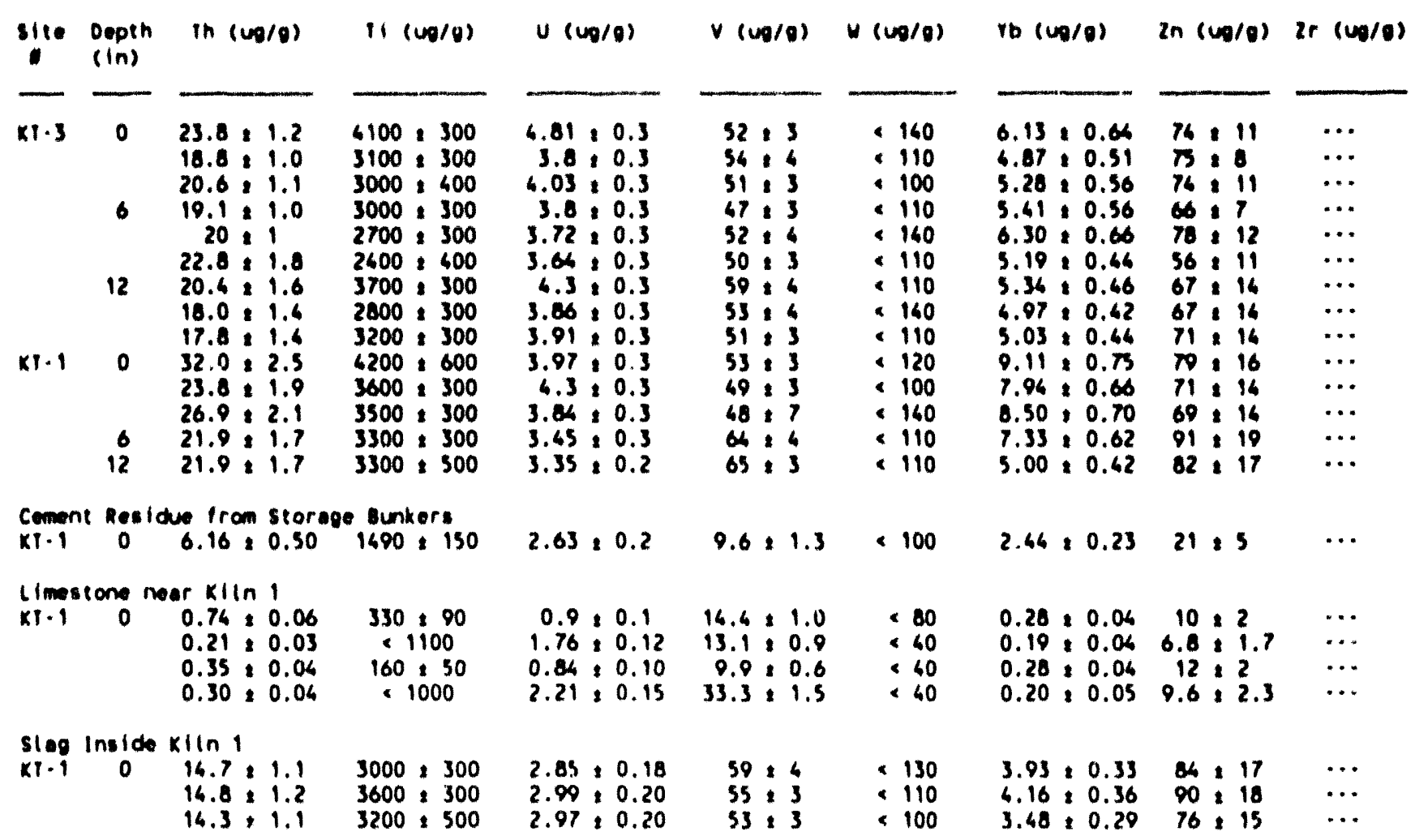




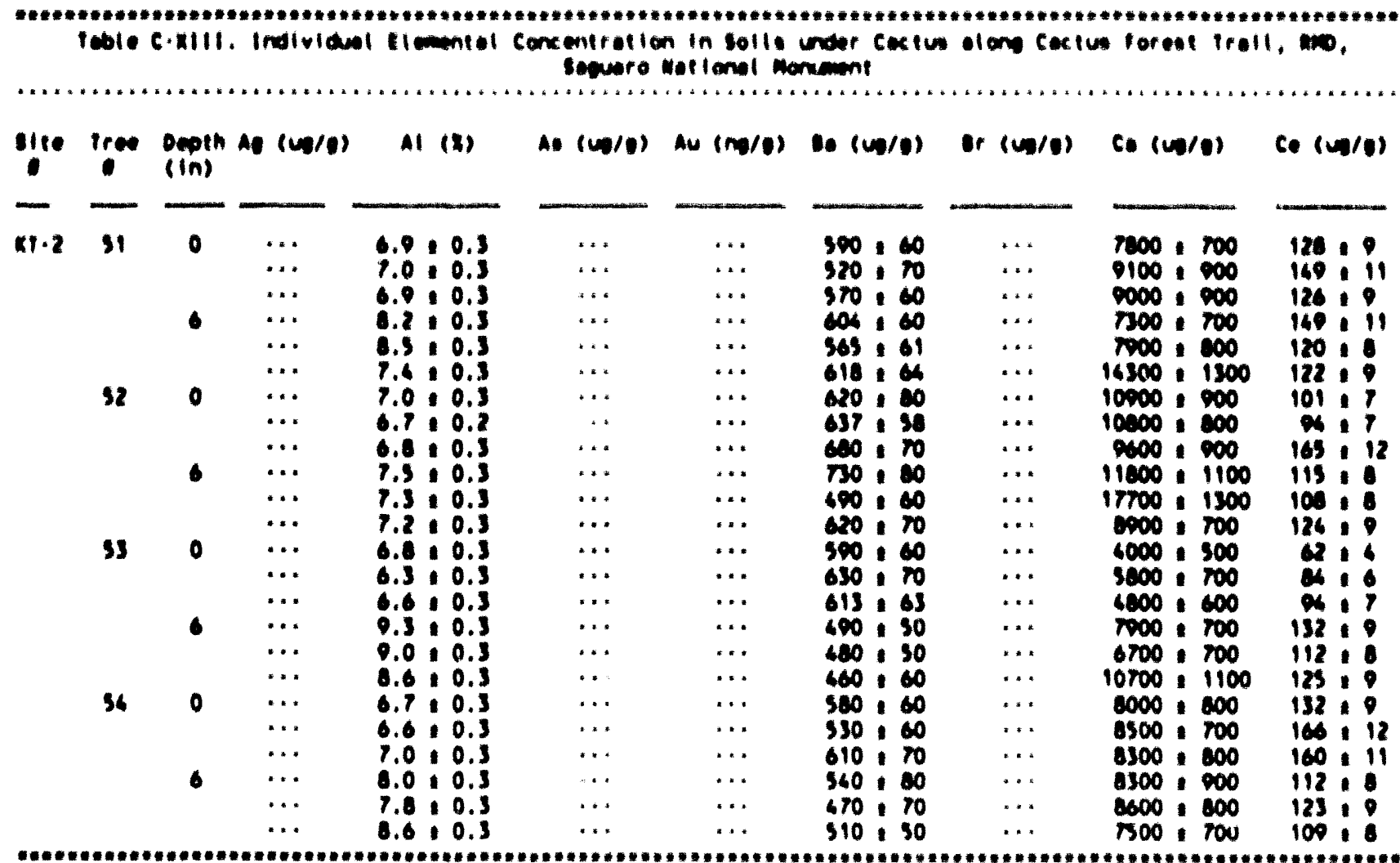

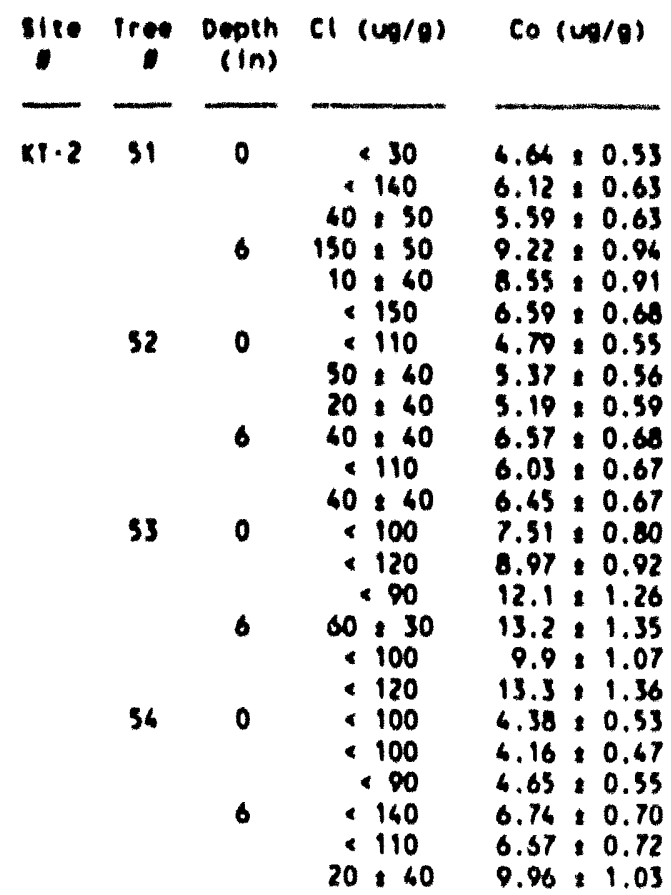

$\cos (x)$

$0.3: 0.2$ $0.5: 0.2$ $0.3: 0.2$ $0.1: 0.1$ $0.3: 0.2$

$0.9: 0.2$

$0.3: 0.2$

$0.2: 0.2$

$0.2: 0.2$

$0.4: 0.2$

$1.0: 0.2$

$0.2: 0.2$

$0.6: 0.2$

$0.3: 0.2$

$0.3: 0.2$

$0.2: 0.2$

$0.2: 0.2$

$0.6: 0.2$

$0.3: 0.2$

$0.3: 0.2$

$0.1: 0.2$

$0.2: 0.2$

$0.6: 0.2$

$0.4: 0.2$ $\operatorname{cr}(u s / \theta)$

$37.0 \div 2.8$ $40.5: 3.6$ $28.6: 2.3$ $63.0: 3.6$ $61.3 \cdot 3.1$

$31.9: 2.7$ $25.1: 2.0$ $27.1 \cdot 2.3$ $30.1: 2.6$ $30.6: 2.6$ $20.0 \times 2.3$ $32.7 \div 2.8$ $30.3 \div 2.9$ $46.0: 3.8$ $46.9 \cdot 3.5$ $52.8 \cdot 4.6$ $53.0: 4.1$ $34.7: 4.6$ $20.2: 2.3$ $29.9: 2.6$ $32.9: 2.7$

$33.3: 2.8$

$33.7: 2.7$ $30.0: 3.3$
C. $(00 / 0)$

Or $(w / \theta)$

lu $(\omega 0 / 0)$

$3.49,0.64$

$6.45: 0.51$

$0.33: 0.49$

$0.36: 0.64$

$0.04: 0.69$

$7.23: 0.56$

$5.36: 0.43$

$5.32,0.62$

$3.64: 0.63$

$5.53,0.42$

$5.91: 0.69$

$6.20,0.48$

$30.9 \cdot 2.21$

$31.9: 2.27$

$32.7 \& 2.34$

$90.6: 6.63$

$95.6: 6.79$

$62.6 \div 4.64$

$3.96: 0.37$

$3.55: 0.31$

$5.17: 0.46$

$5.73: 0.66$

$3.43 \cdot 0.63$

$7.36: 0.58$
$12: 1$

$15.6: 1.3$

$11.0,0.0$

$13.3,1.1$

$12.3: 1.0$

$13.6: 1.1$

$0.9: 0.9$

$12.2: 1.0$

$11.0: 1.1$

$11.2,0.9$

$10.1: 0.9$

$10.7: 0.9$

$5.0: 0.6$

$5.5: 0.5$

$6.4: 0.6$

$7.8: 0.7$

$0.0: 0.8$

$0.7: 0.7$

$0.7: 0.9$

$16.6: 1.6$

$15.6: 1.6$

$12.9: 1.0$

11.8 .1 .0

$0.2: 0.8$
$1.76 \cdot 0.15$ $1.90: 0.17$ $1.66: 0.16$ $2.08: 0.18$ $1.81 \cdot 0.16$ $1.81 \cdot 0.16$ $1.52: 0.13$ $1.32: 0.12$ $1.03: 0.16$ $1.69: 0.15$ $1.66: 0.16$ $1.81: 0.15$ $0.90: 0.09$ $1.11: 0.10$ $1.32: 0.12$ $1.70: 0.16$ $1.71: 0.15$ $2.07: 0.18$ $1.85: 0.16$ $2.02 \cdot 0.17$ $2.12: 0.18$ $1.60: 0.16$ $1.72 \div 0.15$ 


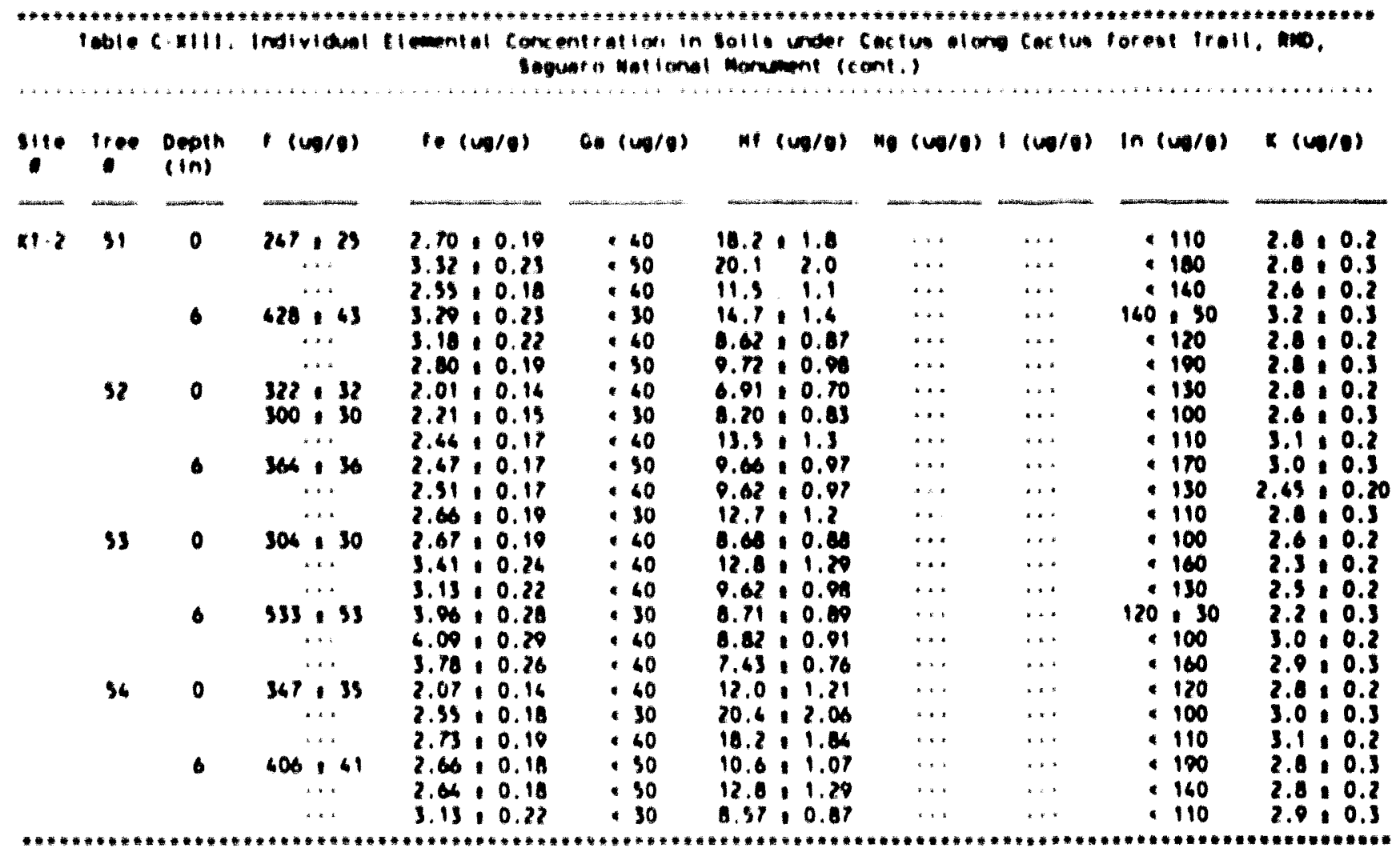

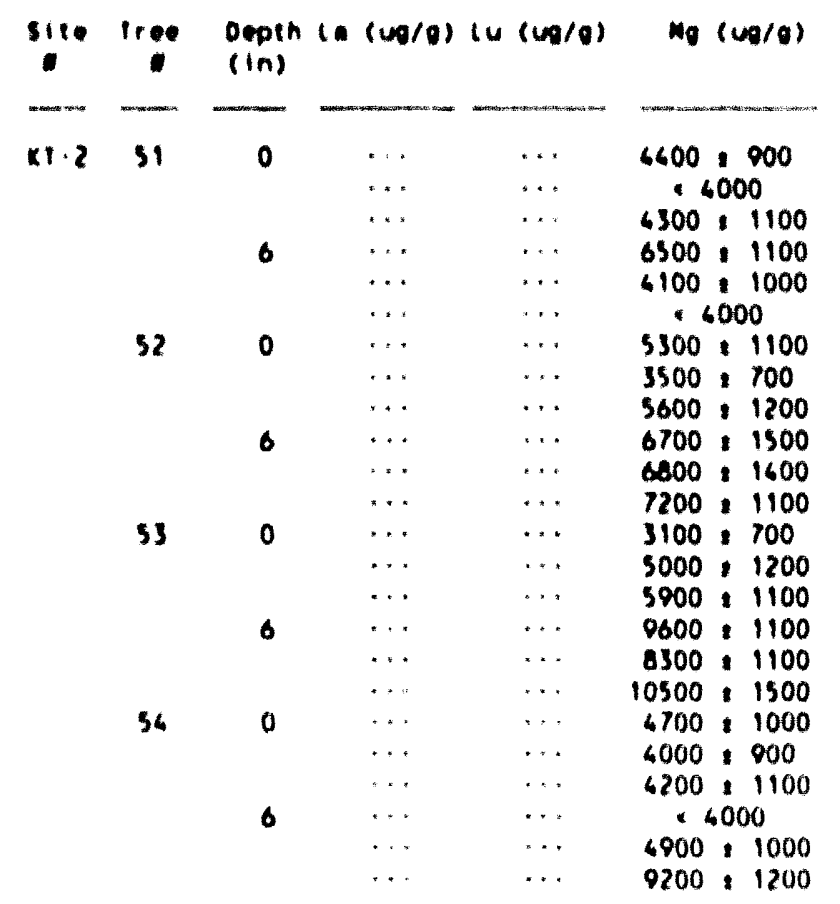

nn $(w / 9)$

490,20

$760: 3 \mathrm{~J}$

$760: 30$

770,30

$680: 30$

$600: 30$

570,30

$630: 30$

$630: 30$

$570: 30$

$580: 30$

$600: 30$

$670: 20$

$620: 30$

$730: 10$

$610: 30$

$4 \times 0: 20$

$020: 30$

$490: 20$

$610: 30$

$640: 30$

$660: 30$

$120: 30$

140,30 no $(00 / 0)$

18200,700

$16700: 600$

$16000: 600$

$13800: 500$

13200,500

15100,500

$17600: 600$

$16800: 600$

$16800: 600$

$17200: 600$

$15300: 500$

15500,500

$12600: 400$

$12000 \div 600$

$10500: 400$

$6700: 200$

7600,300

$7100: 300$

$17800 \div 600$

$17000 \div 600$

$16300: 600$

$15200 \div 600$

$15400 \div 500$

$13100: 400$
Md $($ up/o) py (un/es) ab (ug/g)

\begin{tabular}{|c|c|c|}
\hline$\cdots$ & $7.0: 0.3$ & $118 \cdot 10$ \\
\hline$\cdots$ & $\cdots$ & $126 \cdot 11$ \\
\hline$\cdots$ & $\cdots$ & $111 \cdot 10$ \\
\hline$\cdots$ & $5.3 \times 0.3$ & $136 \cdot 12$ \\
\hline$\cdots$ & $\cdots$ & $144: 12$ \\
\hline$\cdots$ & $\cdots$ & $126 \cdot 11$ \\
\hline$\cdots$ & $7.3: 0.3$ & $120 \cdot 10$ \\
\hline$\cdots$ & $\cdots$ & $116: 10$ \\
\hline$\cdots$ & $\cdots$ & $120 \cdot 11$ \\
\hline$\cdots$ & $0.0: 0.3$ & $121 \times 11$ \\
\hline$\cdots$ & $\cdots$ & $126: 11$ \\
\hline$\cdots$ & $\cdots$ & $127 \cdot 11$ \\
\hline$\cdots$ & $6.2: 0.3$ & $160 \cdot 16$ \\
\hline$\cdots$ & $\cdots$ & $161 \cdot 16$ \\
\hline$\cdots$ & $\cdots$ & $148: 13$ \\
\hline$\cdots$ & $6.6: 0.3$ & $186 \cdot 17$ \\
\hline$\cdots$ & $\cdots$ & $198 * 17$ \\
\hline$\cdots$ & $\cdots$ & $170 \div 15$ \\
\hline$\cdots$ & $5.7: 0.3$ & $121 \cdot 11$ \\
\hline$\cdots$ & $\cdots$ & $117 \cdot 11$ \\
\hline$\cdots$ & $\cdots$ & $109 \cdot 10$ \\
\hline$\cdots$ & $6.6: 0.3$ & $136 \cdot 12$ \\
\hline$\cdots$ & & $\begin{array}{l}126: 11 \\
164: 13\end{array}$ \\
\hline
\end{tabular}


Table C-XIII. Individual Elemental Concentration in Soils under Cactus along Cactus forest Trail, RMD, Saguaro National Monument (cont.)

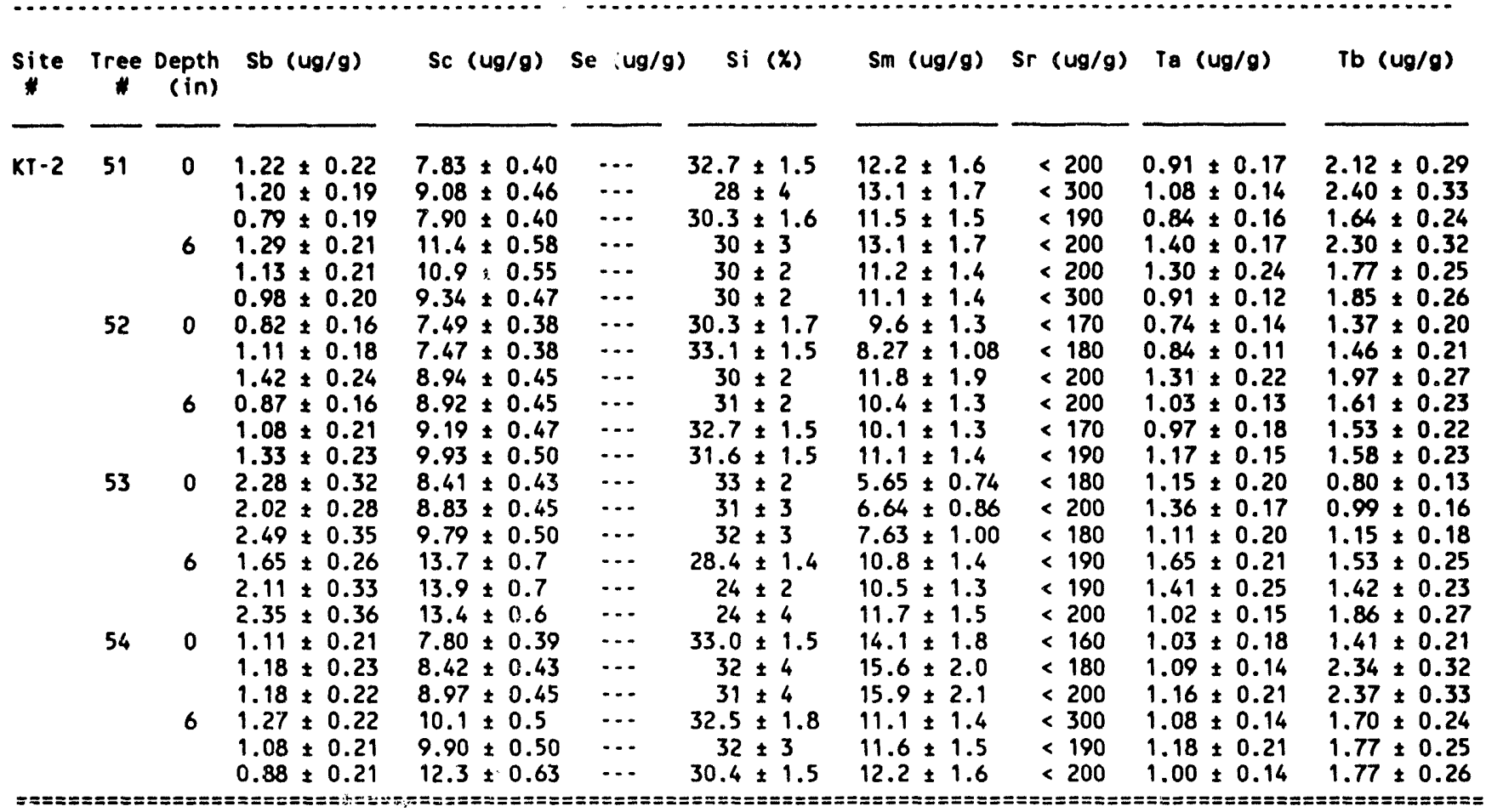

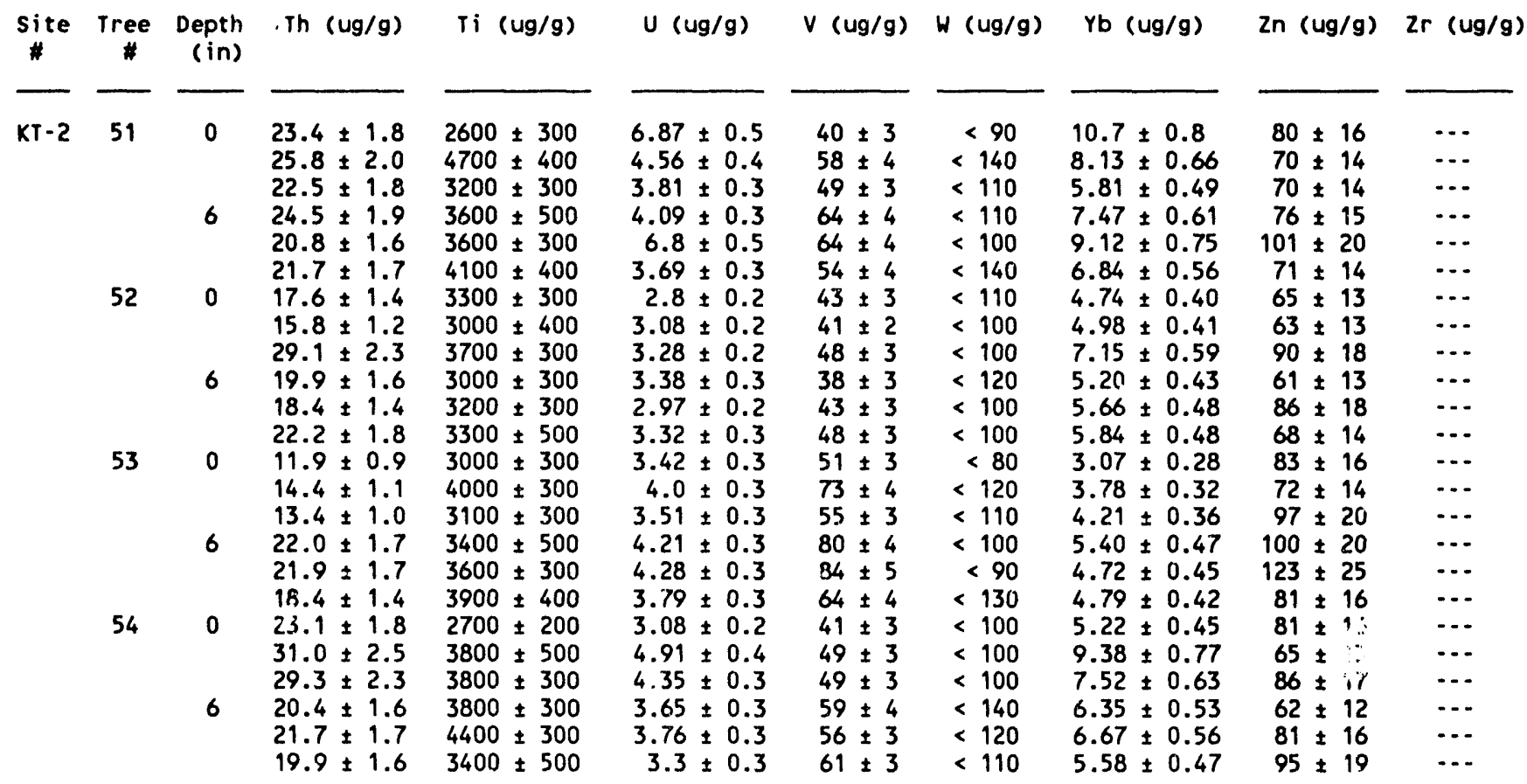

Note: Cacti 51 - 53 appeared to be relatively healthy individuals, while Cactus 54 exhibited substantial browning on the south side coup!ed yith genera! spine loss over the lower portion of the bole. 
Table C-XIV. Individual Elemental Concentration in Solls under North Slope Douglas Fir, RMD,

Saguaro National Monument

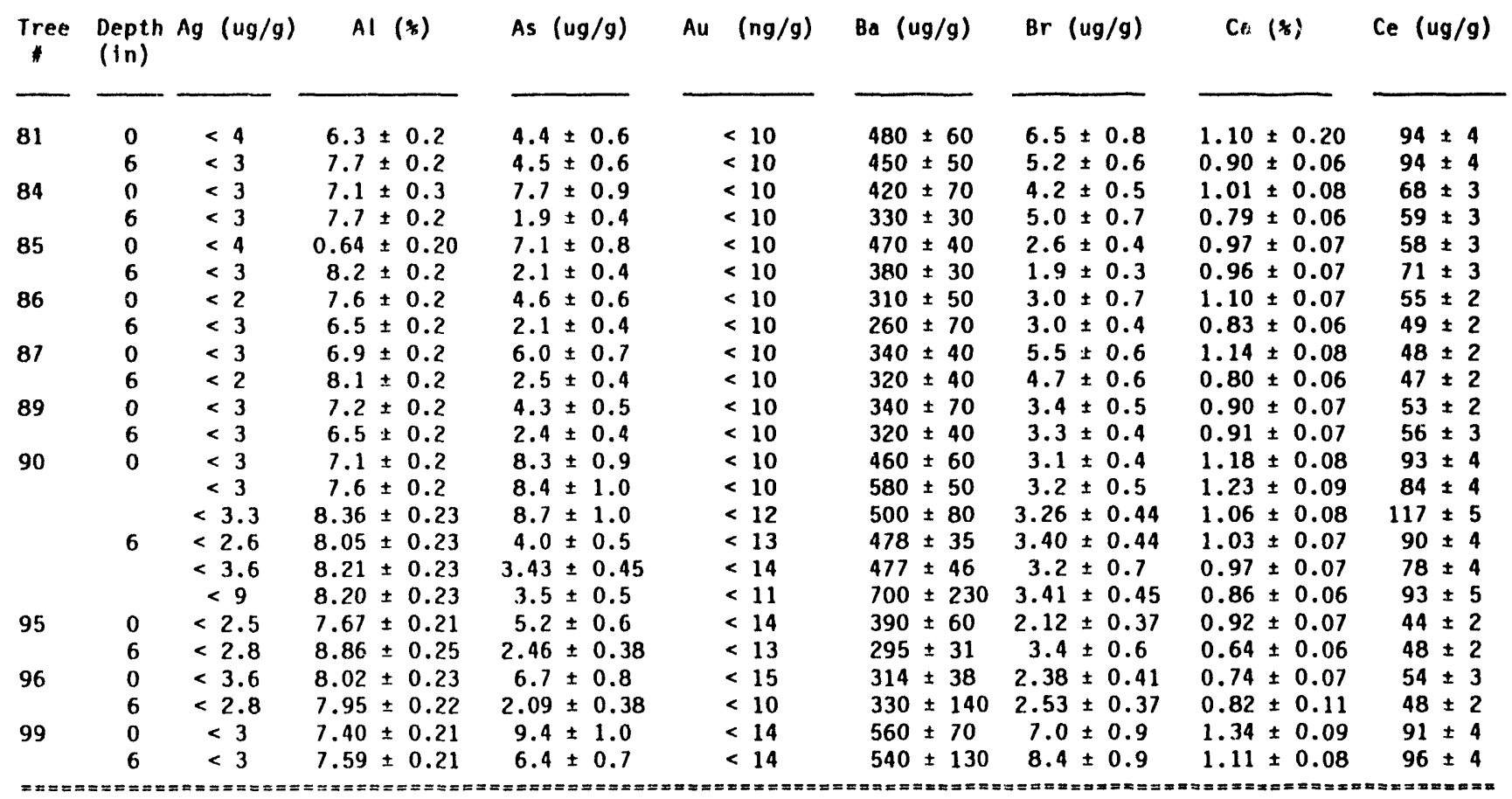

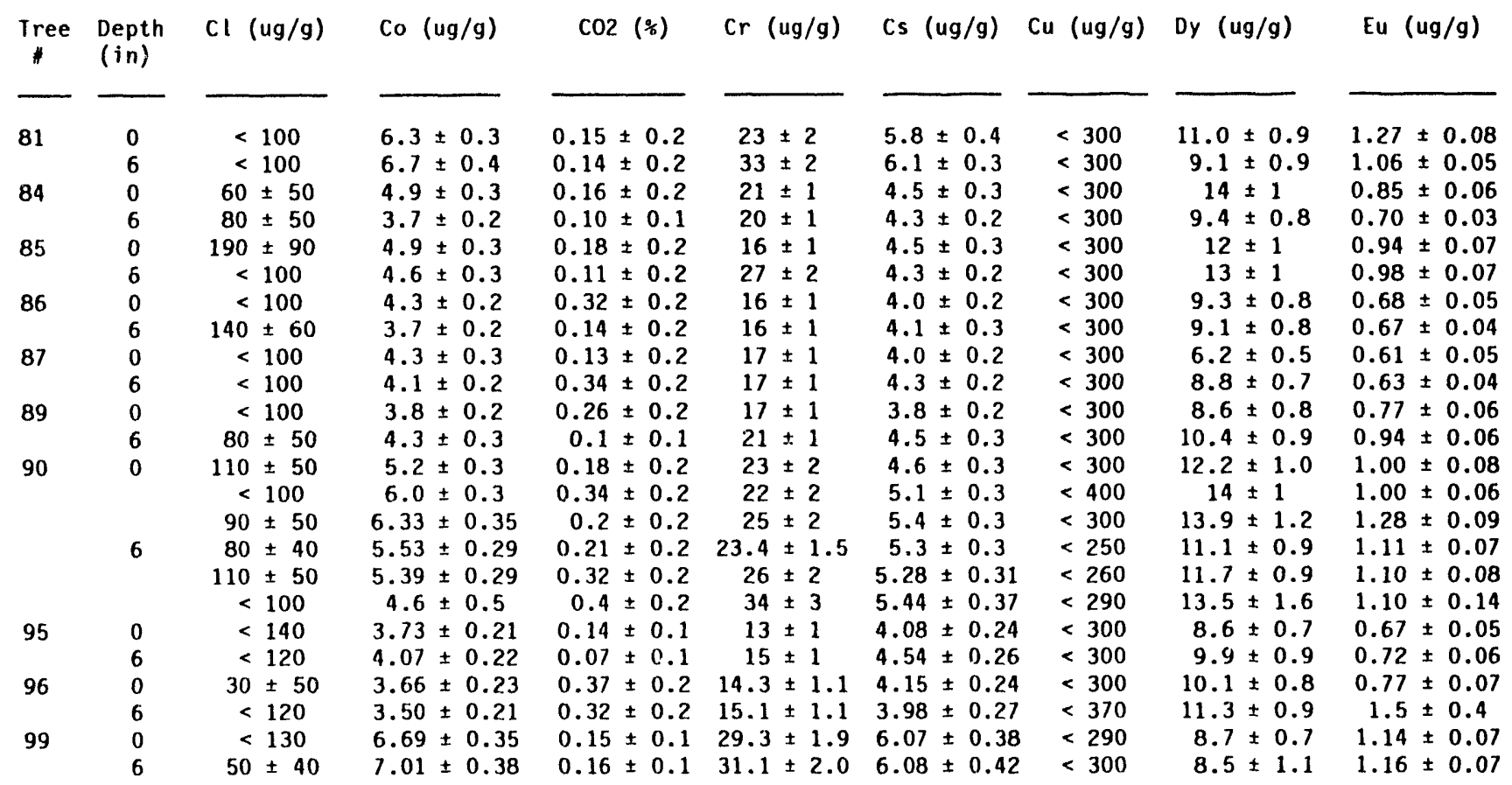




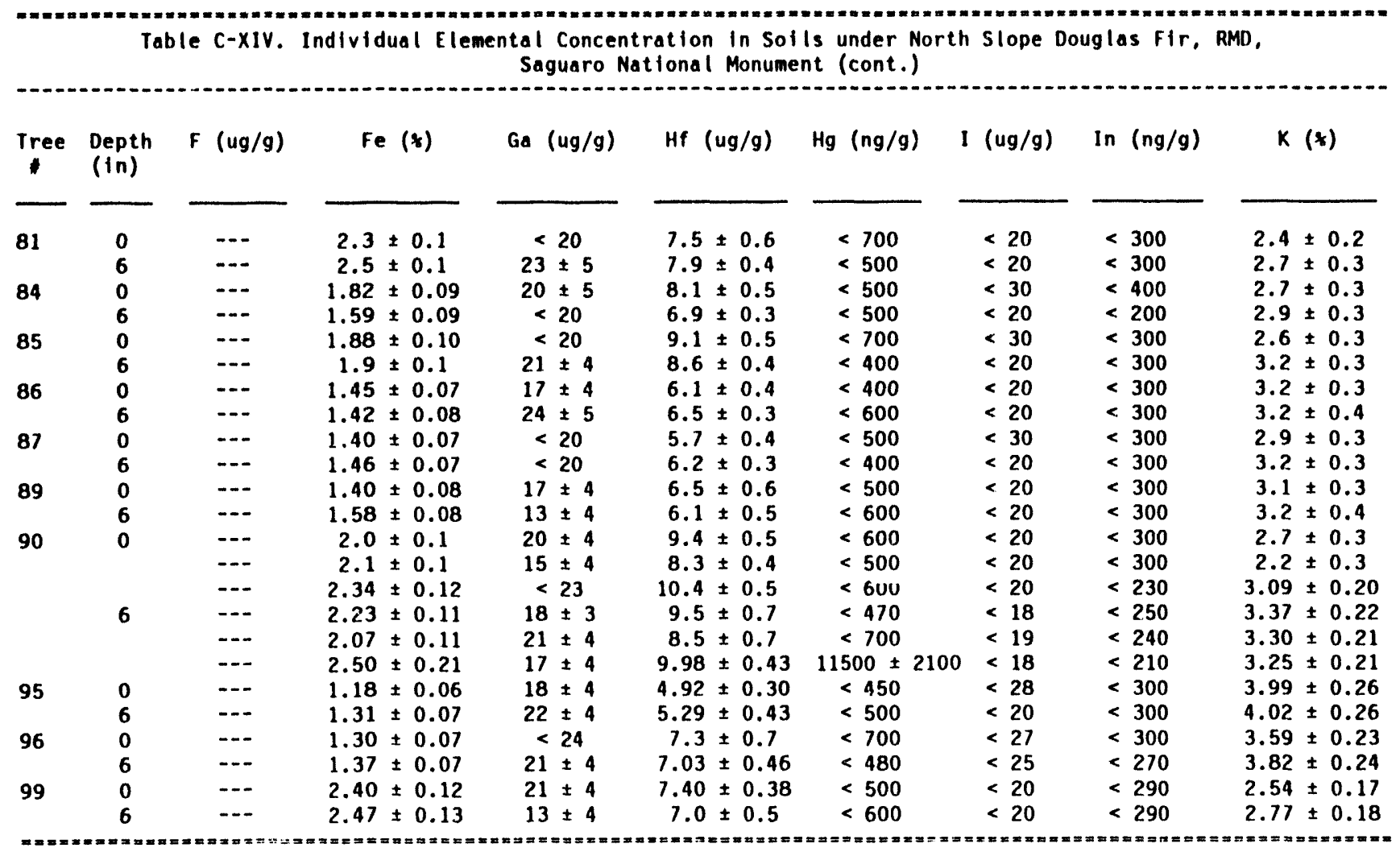

\begin{tabular}{|c|c|c|}
\hline Tree & $\begin{array}{l}\text { Depth } \\
\text { (in) }\end{array}$ & La $(u g / g)$ \\
\hline \multirow[t]{2}{*}{81} & 0 & $46 \pm 3$ \\
\hline & 6 & $13 \pm 2$ \\
\hline \multirow[t]{2}{*}{84} & 0 & $32 \pm 2$ \\
\hline & 6 & $28 \pm 2$ \\
\hline \multirow[t]{2}{*}{85} & 0 & $31 \pm 2$ \\
\hline & 6 & $35 \pm 2$ \\
\hline \multirow[t]{2}{*}{86} & 0 & $26 \pm 1$ \\
\hline & 6 & $24 \pm 1$ \\
\hline \multirow[t]{2}{*}{87} & 0 & $20 \pm 1$ \\
\hline & 6 & $22 \pm 2$ \\
\hline \multirow[t]{2}{*}{89} & 0 & $25 \pm 1$ \\
\hline & 6 & $29 \pm 2$ \\
\hline \multirow[t]{5}{*}{90} & 0 & $40 \pm 2$ \\
\hline & & $\begin{array}{l}38 \pm 2 \\
49 \pm 3\end{array}$ \\
\hline & 6 & $38 \pm 2$ \\
\hline & & $36 \pm 2$ \\
\hline & & $44 \pm 2$ \\
\hline \multirow[t]{2}{*}{95} & 0 & $19 \pm 1$ \\
\hline & 6 & $19 \pm 1$ \\
\hline \multirow[t]{2}{*}{96} & 0 & $24 \pm 1$ \\
\hline & 6 & $22 \pm 1$ \\
\hline \multirow[t]{2}{*}{99} & 0 & $38 \pm 2$ \\
\hline & 6 & $41 \pm 2$ \\
\hline
\end{tabular}

Lu (ng/g)

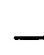

$1140 \pm 60$

$1050 \pm 50$

$2000 \pm 90$

$1540 \pm 70$

$1510 \pm 70$

$1950 \pm 90$

$1300 \pm 60$

$1430 \pm 70$

$960 \pm 50$

$1270 \pm 60$

$1220 \pm 60$

$1450 \pm 70$

$1680 \pm 80$

$1550 \pm 70$

$1580 \pm 80$

$1540 \pm 70$

$1460 \pm 70$

$2060 \pm 100$

$1580 \pm 70$

$1930 \pm 90$

$1780 \pm 90$

$2380 \pm 110$

$868 \pm 43$

$1060 \pm 50$
$M g(u g / g) \quad M n(u g / g)$

$3500 \pm 500$

$5400 \pm 700$

$<3000$

$2900 \pm 700$

$2700 \pm 600$

$2300 \pm 500$

$2900 \pm 600$

$2100 \pm 400$

$1900 \pm 500$

$3400 \pm 600$

$<2000$

$<2000$

$3300 \pm 600$

$3500 \pm 700$

$3400 \pm 600$

$3500 \pm 1000$

$3300 \pm 600$

$3260 \pm 480$

$<3000$

$2100 \pm 600$

$<2200$

$<2200$

$6400 \pm 800$

$4200 \pm 600$
Na $(\%)$

Nd $(u g / g)$

Nd $(u g / g)$

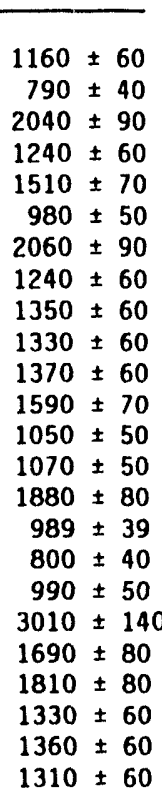

$2.96 \pm 0.08$
$1.94 \pm 0.08$
$1.89 \pm 0.08$
$2.14 \pm 0.10$
$2.14 \pm 0.09$
$2.38 \pm 0.10$
$2.10 \pm 0.09$
$2.25 \pm 0.09$
$1.94 \pm 0.08$
$2.30 \pm 0.10$
$2.16 \pm 0.10$
$2.13 \pm 0.09$
$1.88 \pm 0.08$
$1.99 \pm 0.09$
$2.11 \pm 0.09$
$2.04 \pm 0.07$
$2.07 \pm 0.09$
$2.19 \pm 0.09$
$2.04 \pm 0.09$
$2.21 \pm 0.10$
$2.18 \pm 0.09$
$2.18 \pm 0.09$
$1.71 \pm 0.07$
$1.82 \pm 0.08$

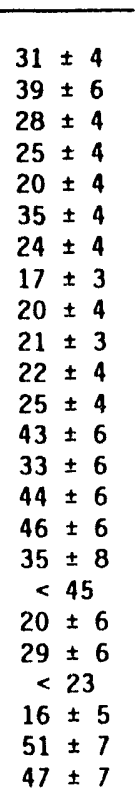



$144 \pm 7$

$149 \pm 7$

$170 \pm 8$

$173 \pm 8$

$154 \pm 8$

$184 \pm 9$

$\begin{array}{ll}-- & 185 \pm 9 \\ -- & 182 \pm 9\end{array}$

$\ldots 181 \pm 9$

$-2 \quad 198 \pm 9$

-- $169 \pm 8$

$--\quad 174 \pm 8$

$\ldots 2 \pm 8$

$--173 \pm 8$

$--193 \pm 10$

-- $193 \pm 9$

$\ldots \quad 179 \pm 9$

$--\quad 202 \pm 13$

$229 \pm 11$

$\ldots 233 \pm 11$

$--\quad 217 \pm 10$

$-\cdots \quad 254 \pm 12$.

$-160 \pm 8$

$\ldots 152 \pm 7$ 


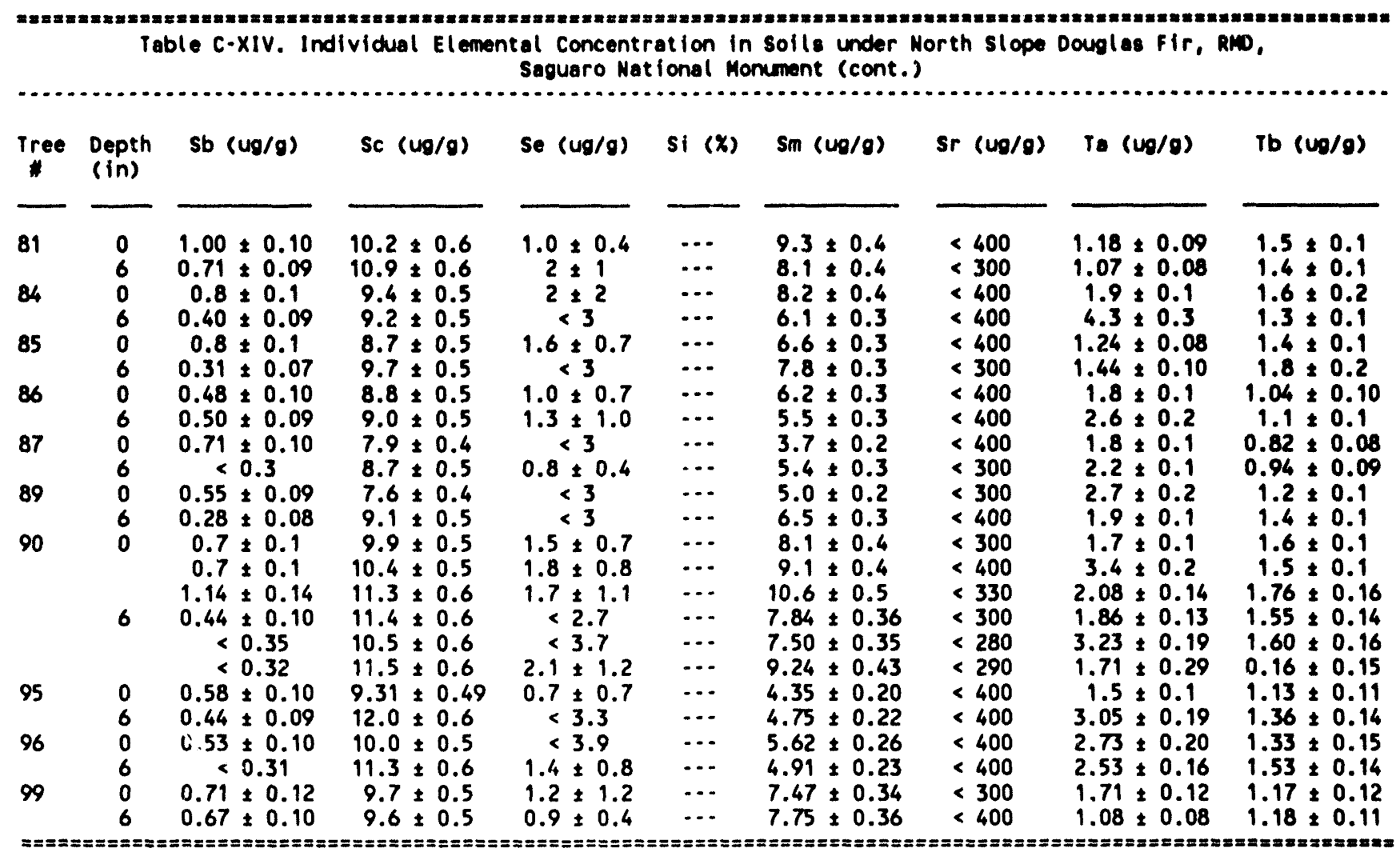

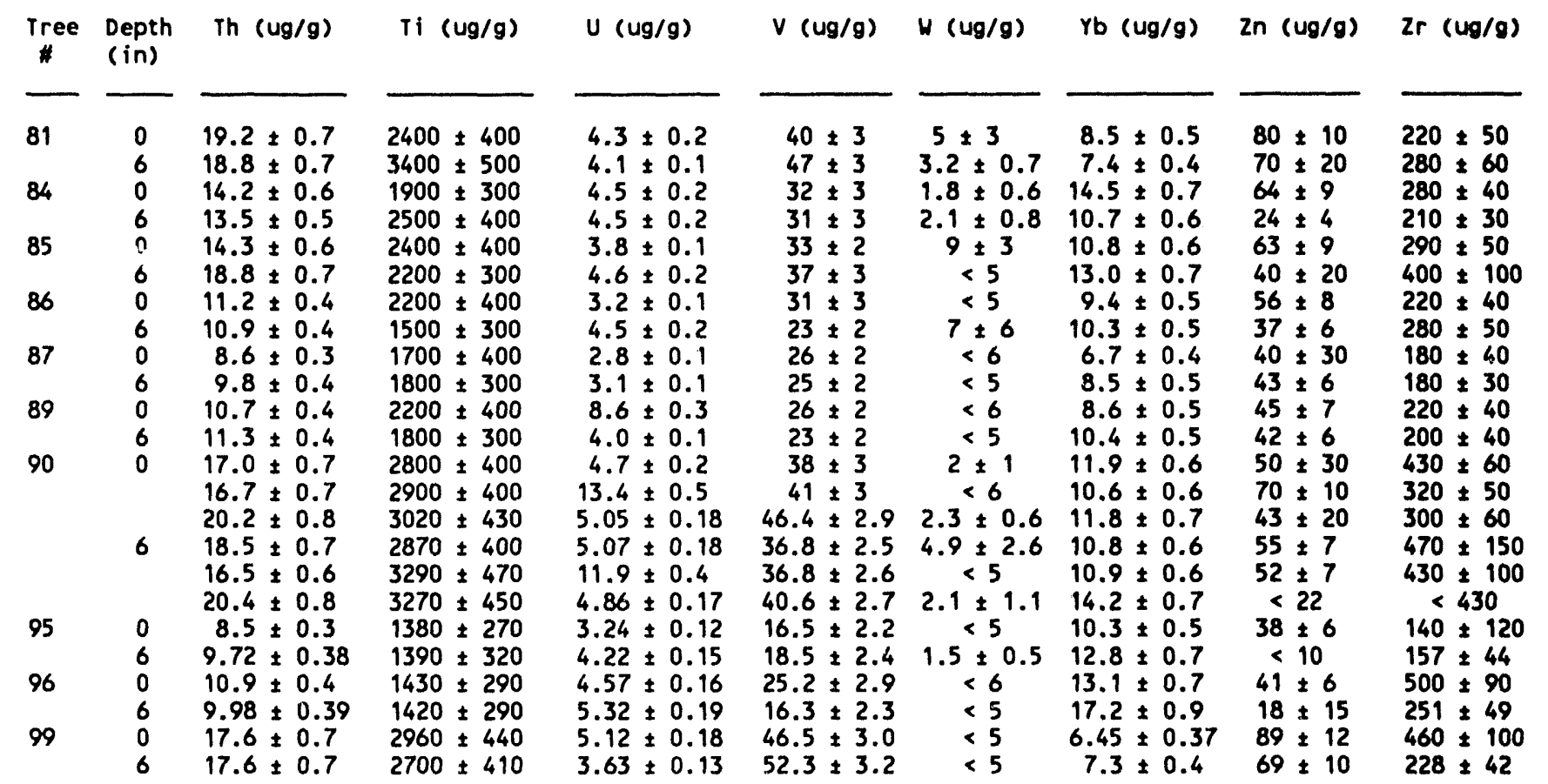




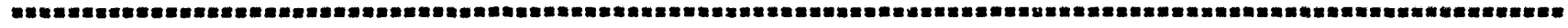
Table C-XV. Individual Elomentel Concentration in soils under Worth slope Ponderose Pine, Riro, Saguaro Mationel Monument

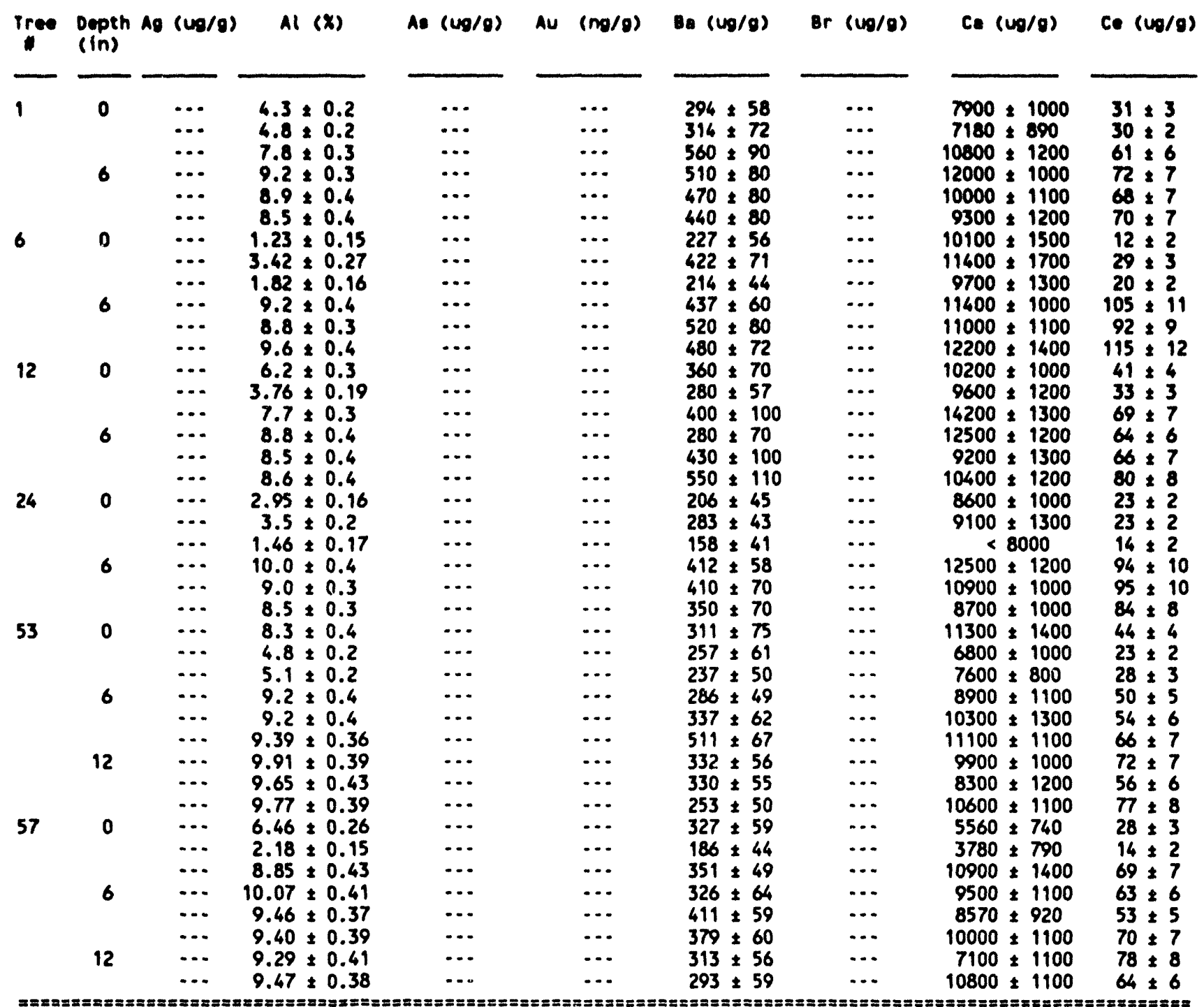

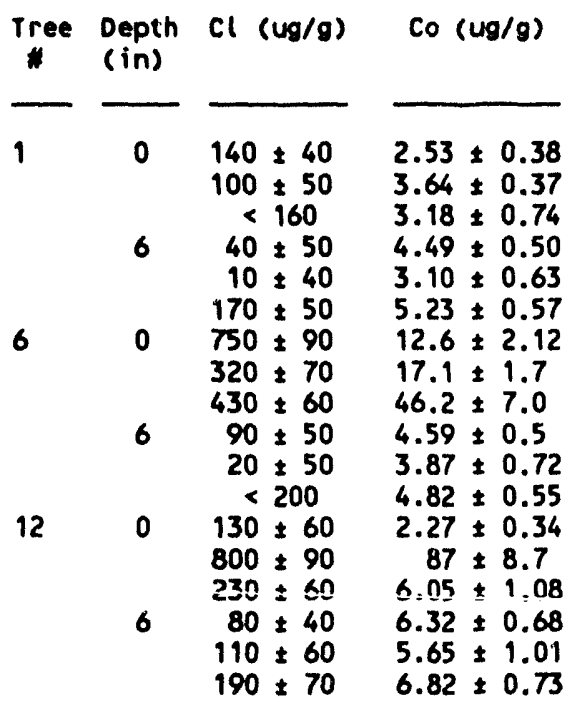

$\operatorname{CO2}(x) \operatorname{Cr}(u g / 9)$

$0.4 \pm 0.2$

$0.9 \pm 0.2$

$0.6 \pm 0.2$

$1.0 \pm 0.2$

$0.6 \pm 0.2$

$0.6 \pm 0.2$

$0.5 \pm 0.2$

$0.5 \pm 0.2$

$1.0 \pm 0.2$

$0.4 \pm 0.2$

$0.8 \pm 0.2$

$0.5 \pm 0.2$

$1.0 \pm 0.2$

$0.4 \pm 0.2$

$0.3 \pm 0.2$

$2.0 \pm 0.2$

$0.5 \pm 0.2$

$0.6 \pm 0.2$
$19.7 \pm 2.0$

$23.1 \pm 1.8$

$21.1 \pm 2.6$

$27.1 \pm 2.2$

$18.0 \pm 2.2$

$22.7 \pm 1.9$

$21.0 \pm 2.6$

$100 \pm 7$

$47.4 \pm 5.0$

$23.7 \pm 2.1$

$24.0 \pm 2.8$

$23.9 \pm 2.1$

$26.8 \pm 2.7$

$12.5 \pm 0.9$

$27.3 \pm 3.1$

$27.9 \pm 2.3$

$25.5 \pm 3.0$

$26.4 \pm 2.2$
Cs $(u g / g)$ Cu $(u g / g)$ Dy $(u g / g)$

Eu (ug/g)

\begin{tabular}{|c|c|c|c|}
\hline $\begin{array}{l}.87 \\
.75 \\
.65 \\
.63\end{array}$ & $\begin{array}{ll} \pm & 0.42 \\
\pm & 0.51 \\
\pm & 0.61 \\
\pm & 0.54 \\
\pm & 0.49 \\
\pm & 0.56 \\
\pm & 0.37 \\
\pm & 0.45 \\
\pm & 0.35 \\
\pm & 0.5 \\
\pm & 0.49 \\
\pm & 0.59 \\
\pm & 0.52\end{array}$ & $\begin{array}{l}\cdots \\
\cdots \\
\cdots \\
\cdots \\
\cdots \\
\cdots \\
\cdots \\
\cdots \\
\cdots \\
\cdots \\
\cdots \\
\cdots \\
\cdots \\
\cdots \\
\cdots \\
\cdots \\
\cdots\end{array}$ & $\begin{aligned} 3.2 & \pm 0.4 \\
4.7 & \pm 0.3 \\
6.3 & \pm 0.6 \\
10.2 & \pm 0.8 \\
9.9 & \pm 0.8 \\
8.2 & \pm 0.8 \\
& \leq 6.2 \\
& \leq 8.7 \\
1.6 & \pm 0.4 \\
& \leq 7 \\
9.9 & \pm 0.9 \\
12.7 & \pm 1.1 \\
19.8 & \pm 1.6 \\
3.7 & \pm 0.5 \\
8.4 & \pm 0.8 \\
8.5 & \pm 0.6 \\
5.2 & \pm 0.6 \\
10.4 & \pm 1.1\end{aligned}$ \\
\hline
\end{tabular}

$0.47 \pm 0.09$

$0.58 \pm 0.10$

$0.87 \pm 0.11$

$1.19 \pm 0.14$

$1.06 \pm 0.11$

$0.83 \pm 0.10$

$0.28 \pm 0.09$

$0.47 \pm 0.09$

$0.28 \pm 0.08$

$1.23 \pm 0.15$

$1.28 \pm 0.13$

$1.32 \pm 0.16$

$0.62 \pm 0.08$

$0.44 \pm 0.09$

$0.80 \pm 0.10$

0.89 $\div 0.11$

$0.82 \pm 0.10$

-85 . 


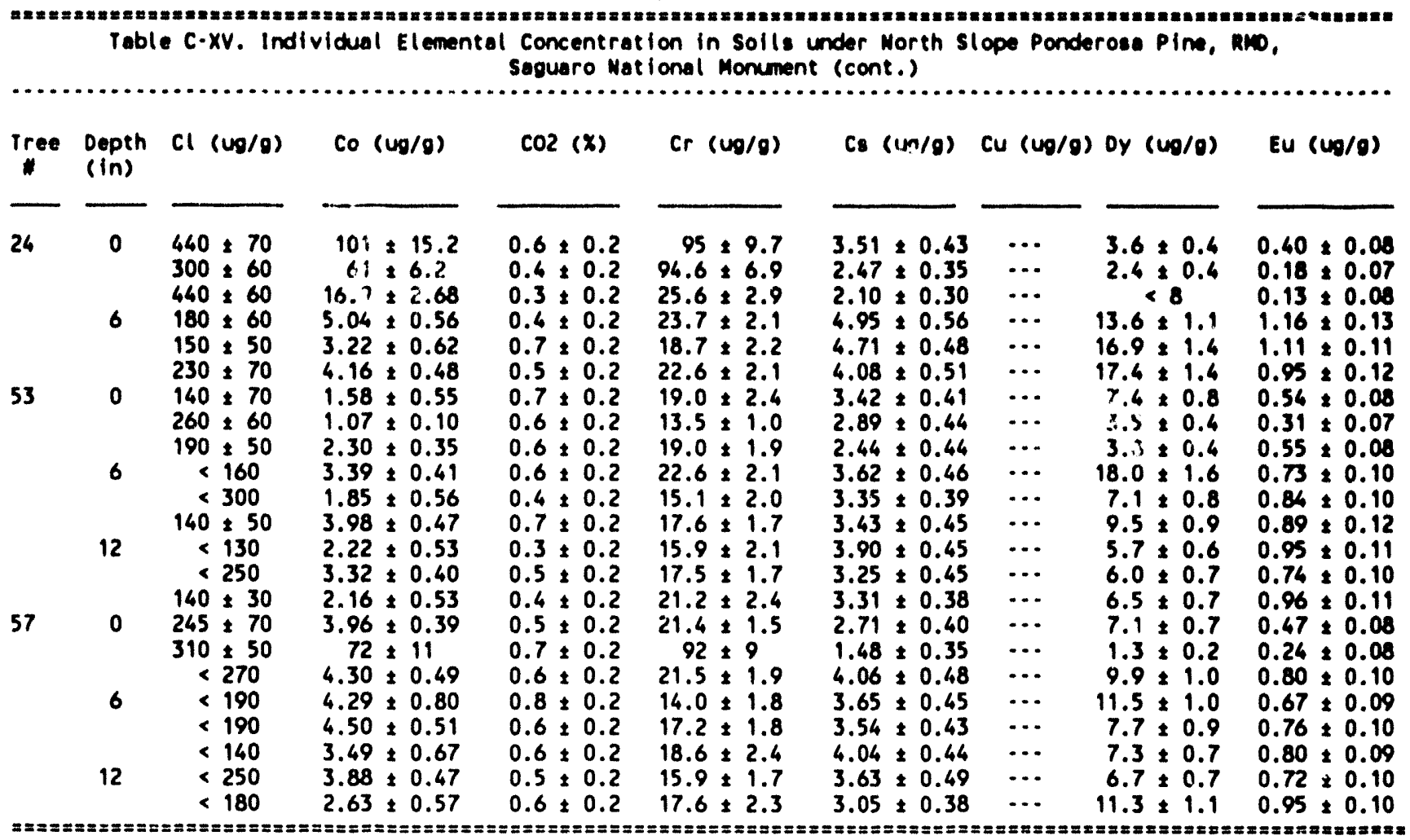

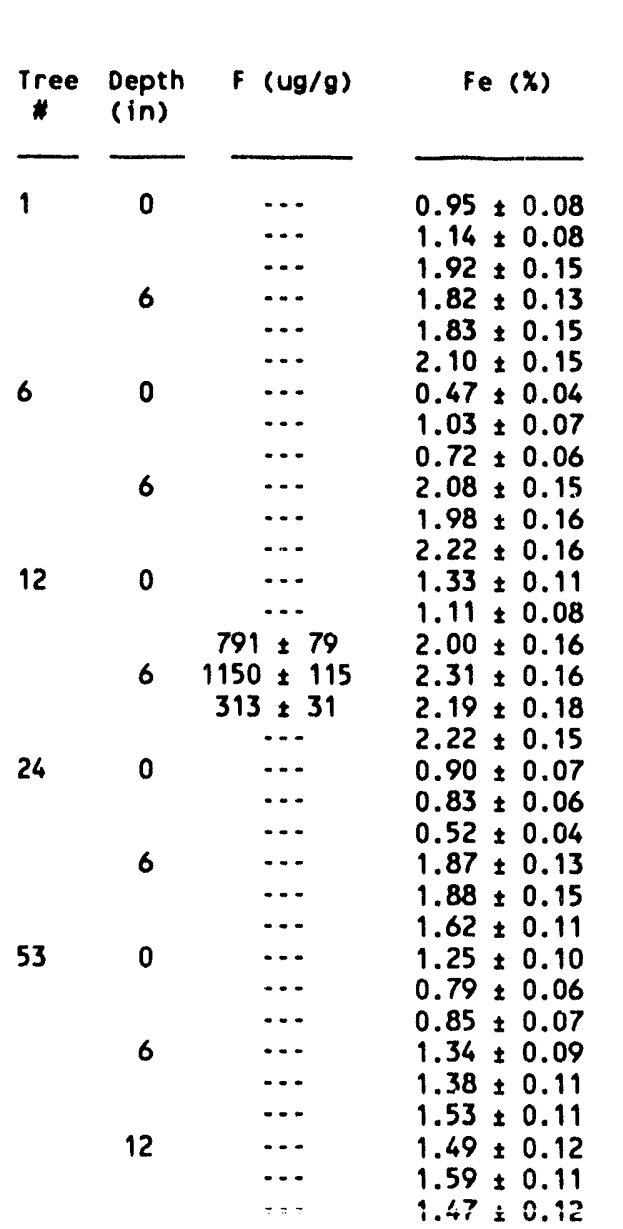

Ga $(u g / g)$

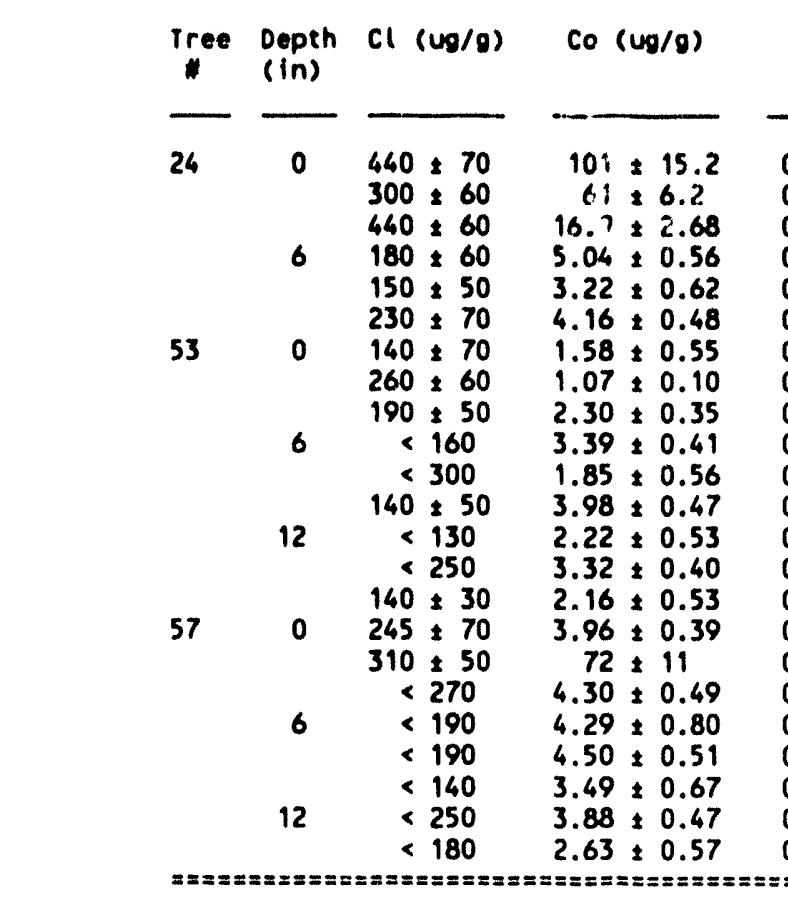

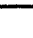

$<58$
$<50$
$<49$
$<40$
$<60$
$<70$
$<66$
$<85$

$<58$

$<50$

$<60$

$<70$

$<60$

$<53$

$<60$

$<70$

$<80$

$<80$

$<40$

$<80$

$<60$

$<70$

$<50$

$<70$

$<57$

$<57$

$<40$

$<80$

$<80$

$<54$

$<56$

$<85$

< só

\begin{abstract}
Hf $(u g / g)$
\end{abstract}

\begin{tabular}{l} 
\\
\hline $13.6 \pm 1.13$ \\
$17.8 \pm 1.81$ \\
$6.78 \pm 0.58$ \\
$8.73 \pm 0.72$ \\
$6.61 \pm 0.56$ \\
$7.66 \pm 0.63$ \\
$1.24 \pm 0.16$ \\
$2.89 \pm 0.27$ \\
$3.51 \pm 0.34$ \\
$9.36 \pm 0.77$ \\
$8.04 \pm 0.66$ \\
$7.74 \pm 0.64$ \\
$10.6 \pm 0.87$ \\
$2.42 \pm 0.26$ \\
$12.9 \pm 1.07$ \\
$5.82 \pm 0.49$ \\
$5.53 \pm 0.47$ \\
$6.69 \pm 0.56$ \\
$4.47 \pm 0.42$ \\
$2.71 \pm 0.25$ \\
$1.88 \pm 0.21$ \\
$7.61 \pm 0.63$ \\
$9.18 \pm 0.76$ \\
$6.35 \pm 0.53$ \\
$3.74 \pm 0.34$ \\
$3.67 \pm 0.33$ \\
$7.77 \pm 0.67$ \\
$6.47 \pm 0.54$ \\
$5.01 \pm 0.43$ \\
$4.04 \pm 0.36$ \\
$4.09 \pm 0.36$ \\
$5.98 \pm 0.51$ \\
$6.63 \pm 0.55$
\end{tabular}

$\mathrm{Hg}(u g / g)$ I (ug/g)

$\ln (n g / g)$

$K(x)$

\begin{tabular}{|c|c|c|}
\hline$\ldots$ & $\ldots$ & $<140$ \\
\hline$\cdots$ & $\ldots$ & $<150$ \\
\hline$\cdots$ & $\cdots$ & $<190$ \\
\hline$\cdots$ & $\cdots$ & $<150$ \\
\hline$\cdots$ & $\cdots$ & $<160$ \\
\hline$\cdots$ & $\cdots$ & $<300$ \\
\hline$\cdots$ & $\cdots$ & $<180$ \\
\hline$\cdots$ & $\cdots$ & $<300$ \\
\hline$\cdots$ & $\cdots$ & $<170$ \\
\hline$\cdots$ & $\cdots$ & $<170$ \\
\hline$\cdots$ & $\cdots$ & $<150$ \\
\hline$\cdots$ & $\cdots$ & $<300$ \\
\hline$\cdots$ & $\cdots$ & $<180$ \\
\hline$\cdots$ & $\cdots$ & $<190$ \\
\hline$\cdots$ & $\cdots$ & $<200$ \\
\hline$\cdots$ & $\cdots$ & $<180$ \\
\hline$\cdots$ & $\cdots$ & $<300$ \\
\hline$\cdots$ & $\cdots$ & $<200$ \\
\hline$\cdots$ & $\cdots$ & $<130$ \\
\hline$\ldots$ & $\cdots$ & $<190$ \\
\hline$\cdots$ & $\cdots$ & $<200$ \\
\hline$\cdots$ & $\cdots$ & $<200$ \\
\hline$\cdots$ & $\cdots$ & $<160$ \\
\hline$\cdots$ & $\cdots$ & $<170$ \\
\hline$\cdots$ & $\cdots$ & $<210$ \\
\hline$\cdots$ & $\cdots$ & $<180$ \\
\hline$\cdots$ & $\cdots$ & $<130$ \\
\hline$\cdots$ & $\cdots$ & $<200$ \\
\hline$\cdots$ & $\cdots$ & $<300$ \\
\hline$\cdots$ & $\cdots$ & $<180$ \\
\hline$\cdots$ & $\ldots$ & $<150$ \\
\hline$\cdots$ & $\cdots$ & $<320$ \\
\hline$\cdots$ & - . & $=200$ \\
\hline
\end{tabular}

$1.92 \div 0.28$

$2.12 \pm 0.21$

$2.64 \div 0.31$

$3.4 \div 0.4$

$3.6 \div 0.3$

$2.8 \div 0.3$

$<0.54$

$1.80 \pm 0.33$

$0.73 \pm 0.17$

$2.7 \pm 0.3$

$3.0 \pm 0.3$

$3.3 \pm 0.3$

$3.1 \pm 0.3$

$1.37 \pm 0.20$

$2.8 \pm 0.4$

$2.7 \pm 0.3$

$2.4 \pm 0.3$

$2.7 \pm 0.3$

$1.7 \div 0.2$

$1.3 \pm 0.2$

$<0.8$

$3.3 \pm 0.3$

$3.6 \pm 0.4$

$3.1 \pm 0.3$

$2.33 \pm 0.29$

$1.66 \pm 0.21$

$2.01 \pm 0.24$

$2.7 \pm 0.3$

$2.8 \pm 0.3$

$3.02 \pm 0.36$

$3.40 \pm 0.27$

$2.83 \pm 0.35$

$3.09+0.27$ 
Table C-XY. Individul Elementel Concentration In solls under North slope Ponderose Pine, Aro. Saguaro National Monument (cont.)

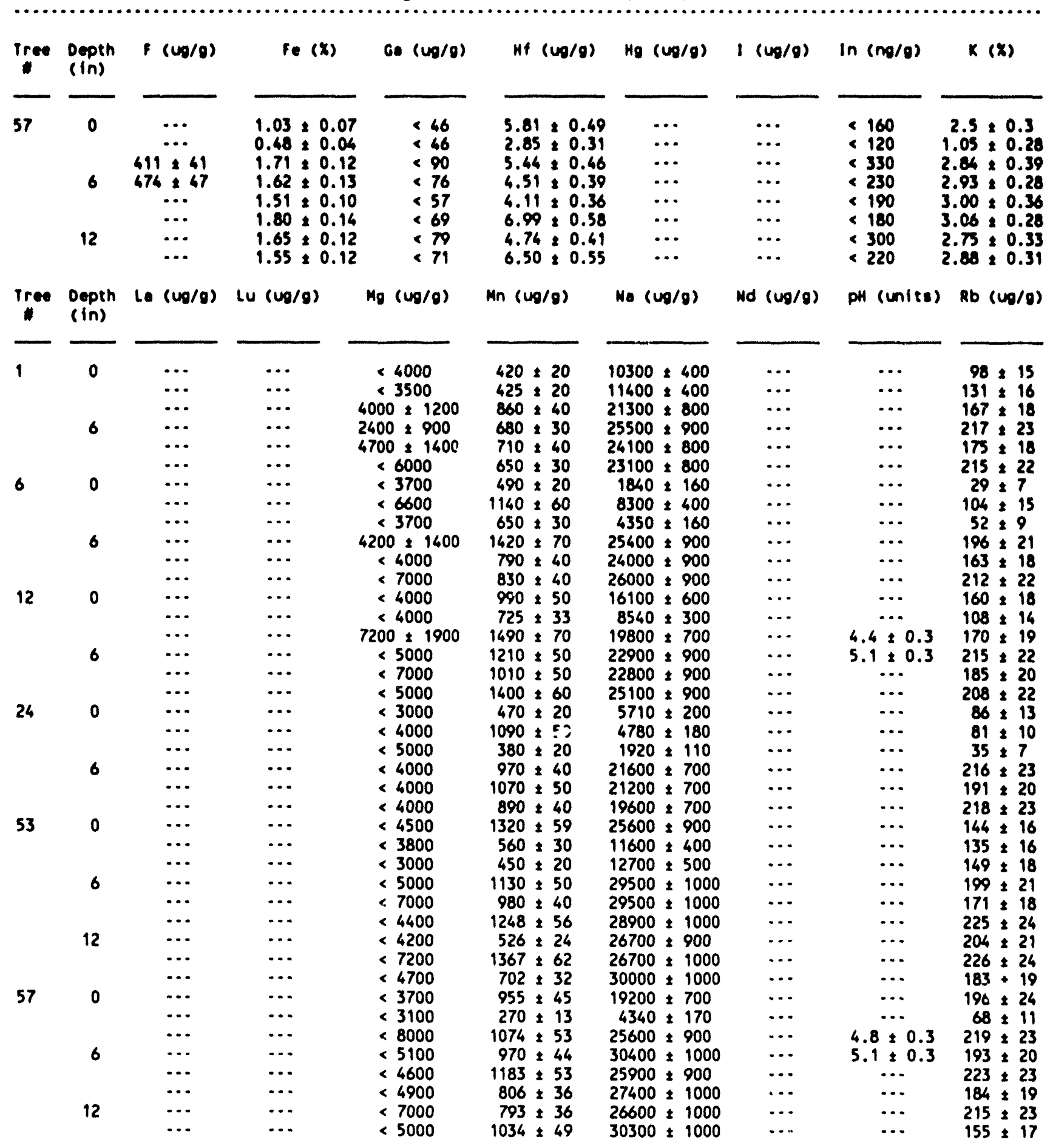




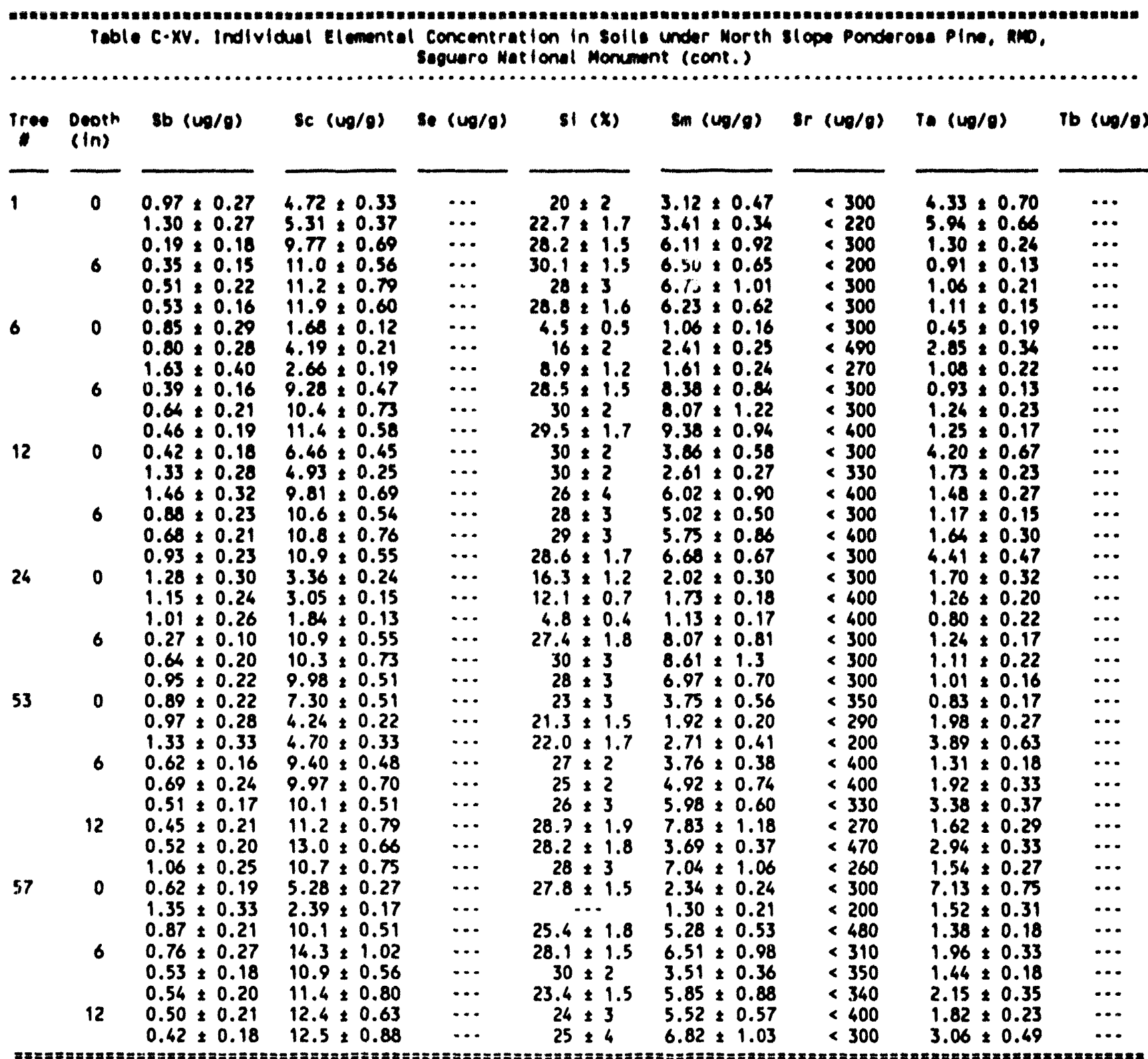

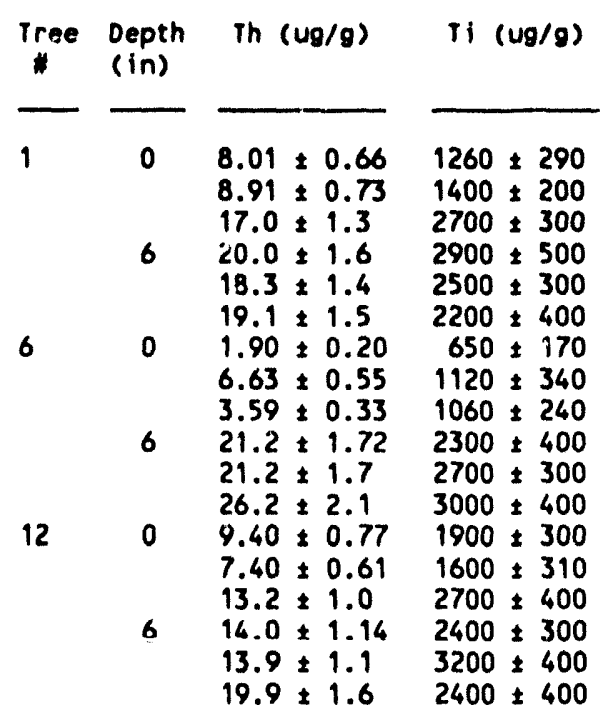

\begin{tabular}{|c|c|c|}
\hline$U(u g / g)$ & $V(u g / g)$ & $W(u g / g)$ \\
\hline $\begin{array}{l}2.05 \pm 0.2 \\
2.01 \pm 0.2 \\
4.55 \pm 0.3 \\
4.16 \pm 0.3 \\
3.58 \pm 0.3 \\
3.57 \pm 0.3 \\
0.63 \pm 0.1 \\
1.82 \pm 0.15 \\
1.07 \pm 0.1 \\
4.68 \pm 0.4 \\
5.17 \pm 0.4 \\
5.72 \pm 0.5 \\
2.58 \pm 0.2 \\
1.01 \pm 0.1 \\
3.67 \pm 0.3 \\
4.38 \pm 0.4 \\
7.62 \pm 0.6 \\
4.38 \pm 0.4\end{array}$ & $\begin{aligned} 14.6 & \pm 2.5 \\
16 & \pm 2 \\
32 & \pm 3 \\
34 & \pm 3 \\
33 & \pm 3 \\
24 & \pm 3 \\
9.6 & \pm 1.4 \\
17.8 & \pm 2.9 \\
17 & \pm 2 \\
36 & \pm 3 \\
28 & \pm 2 \\
42 & \pm 4 \\
22 & \pm 2 \\
20 & \pm 2 \\
37 & \pm 3 \\
36 & \pm 3 \\
36 & \pm 4 \\
35 & \pm 3\end{aligned}$ & $\begin{aligned} & 1700 \pm 200 \pm 400 \\
& 2900 \pm 400<170 \\
&<130 \\
&<130 \\
&<180 \\
&<230 \\
& 1990 \pm 230 \\
& 408 \pm 63 \\
&<180 \\
&<130 \\
&<200 \\
& 1700 \pm 200 \\
& 840 \pm 50 \\
&<200 \\
&<160 \\
&<200 \\
&<200\end{aligned}$ \\
\hline
\end{tabular}

Yb (ug/g)

2n $(40 / g)$

2r (ug/g) 


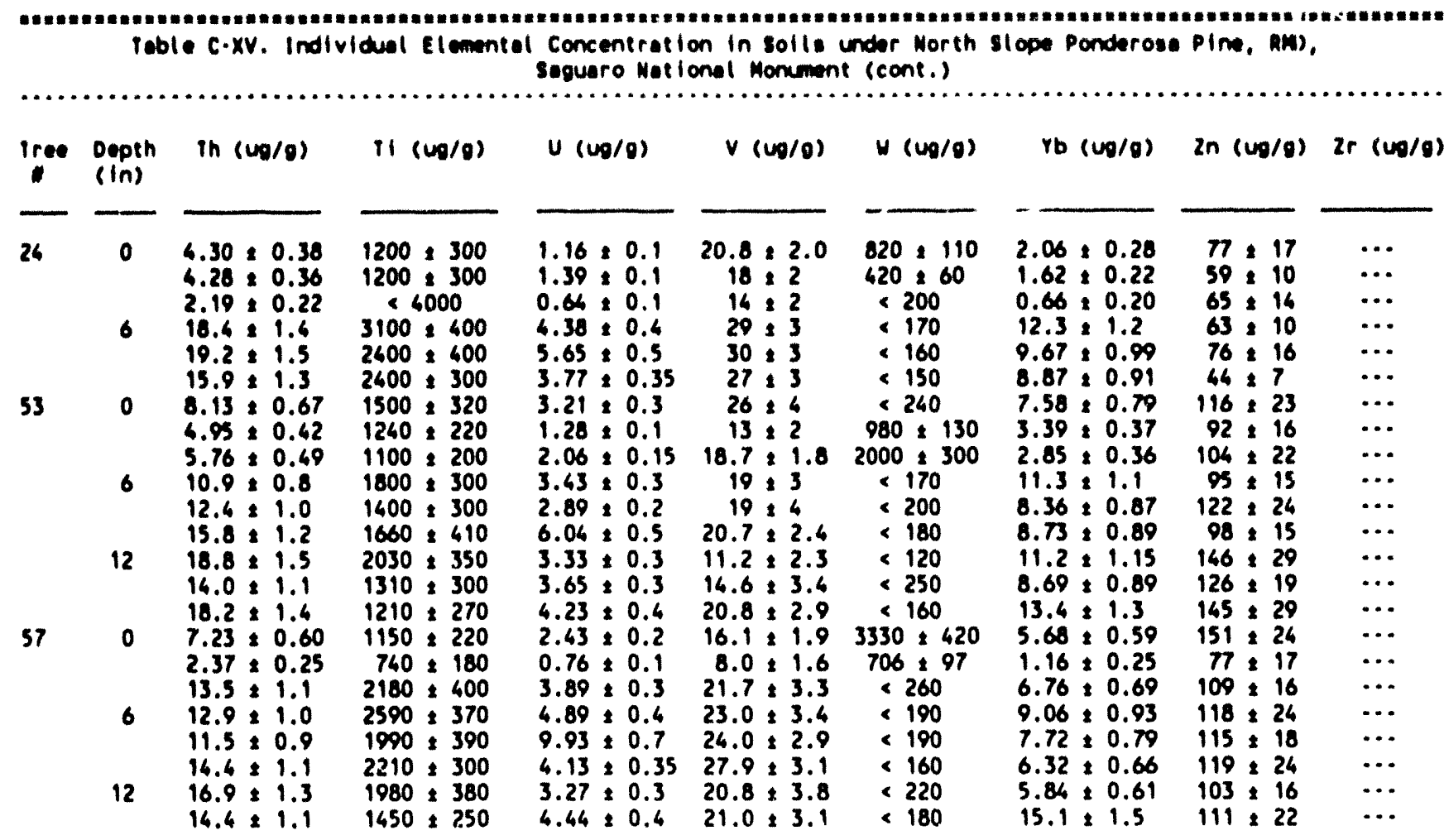

Note: Irees showing severe growth depression. 2, 2.5, 3, 4, 10, 11, 29, 32, 34, 34', 35, 36, (6). 


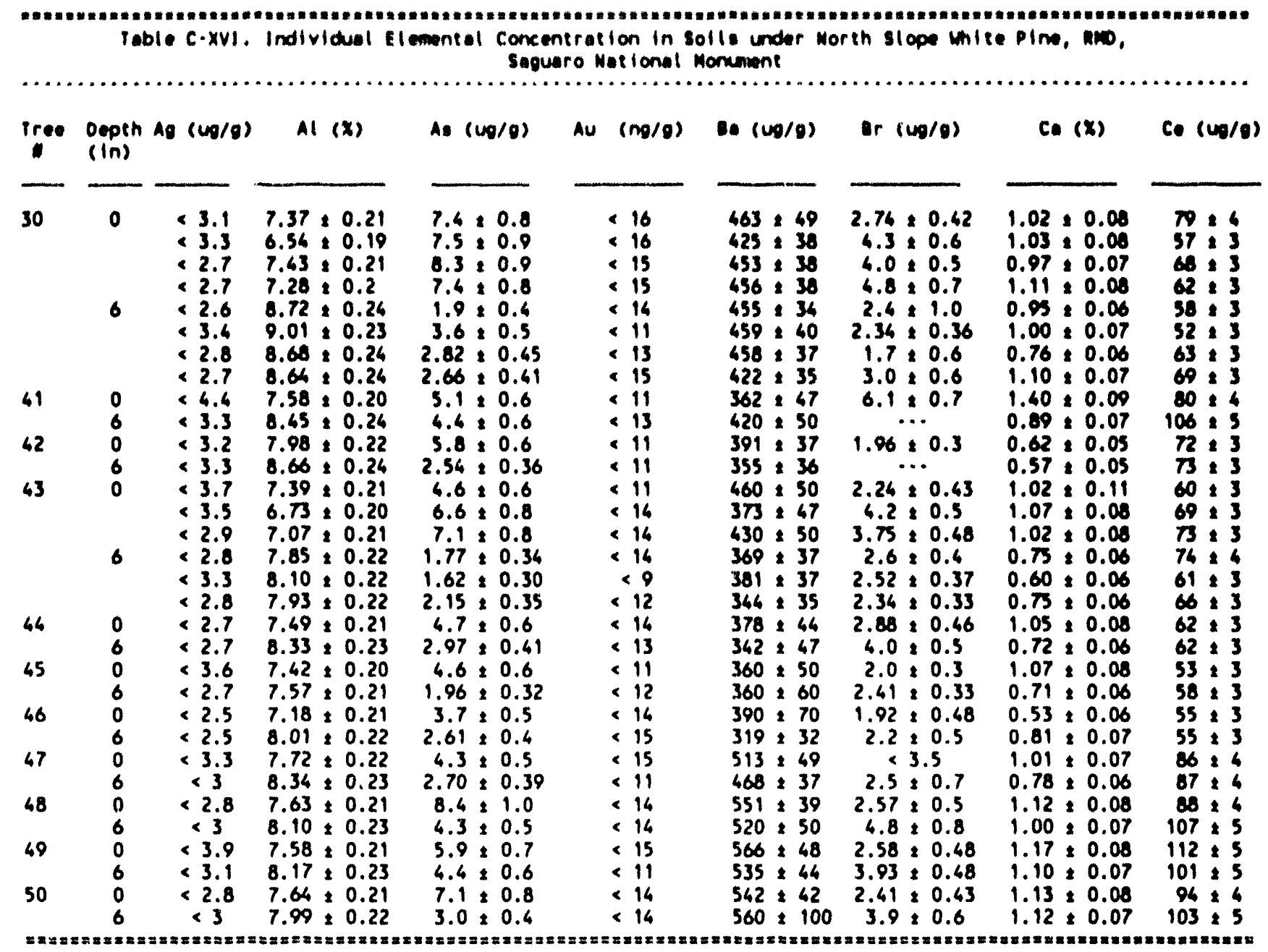

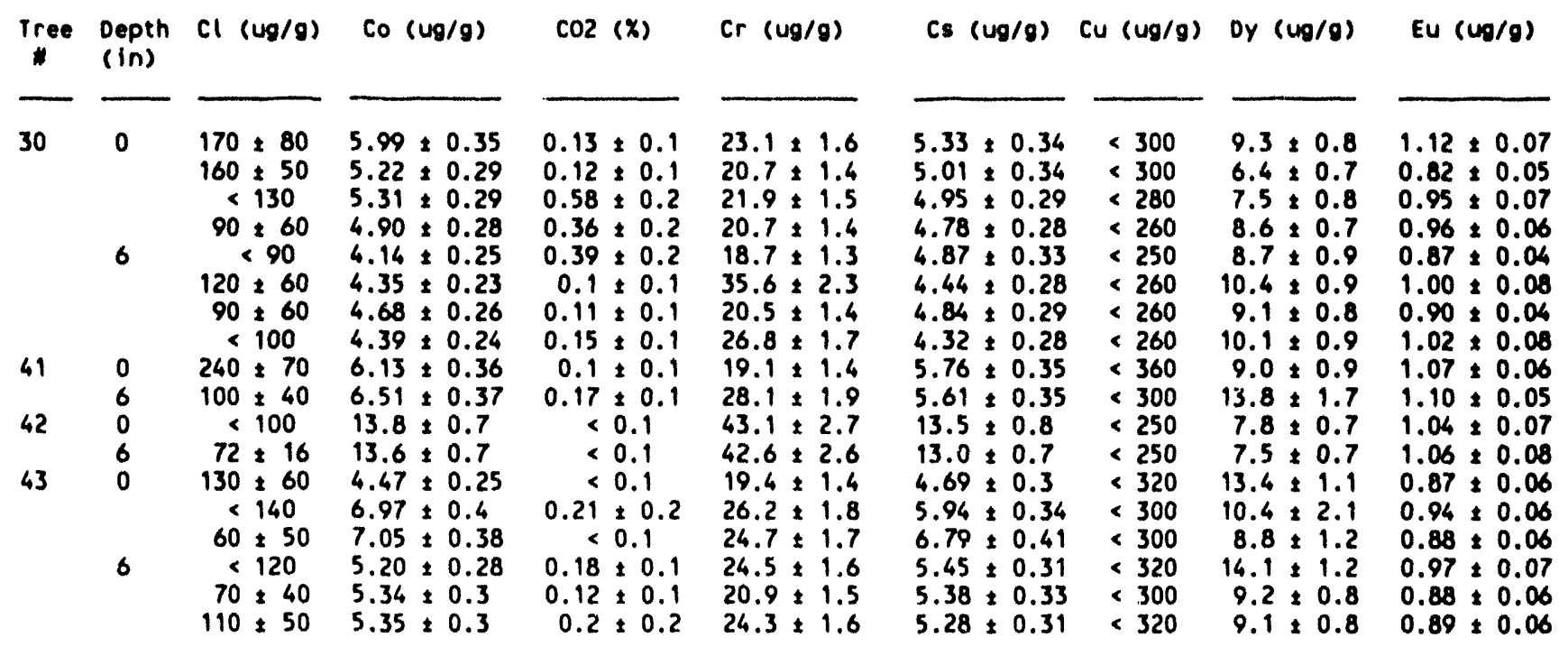


rable C.XYI. Indivitual tiementel Concentration in solle under Morth slope thlte pine, aro,

sequero Wational Monument (cont.)

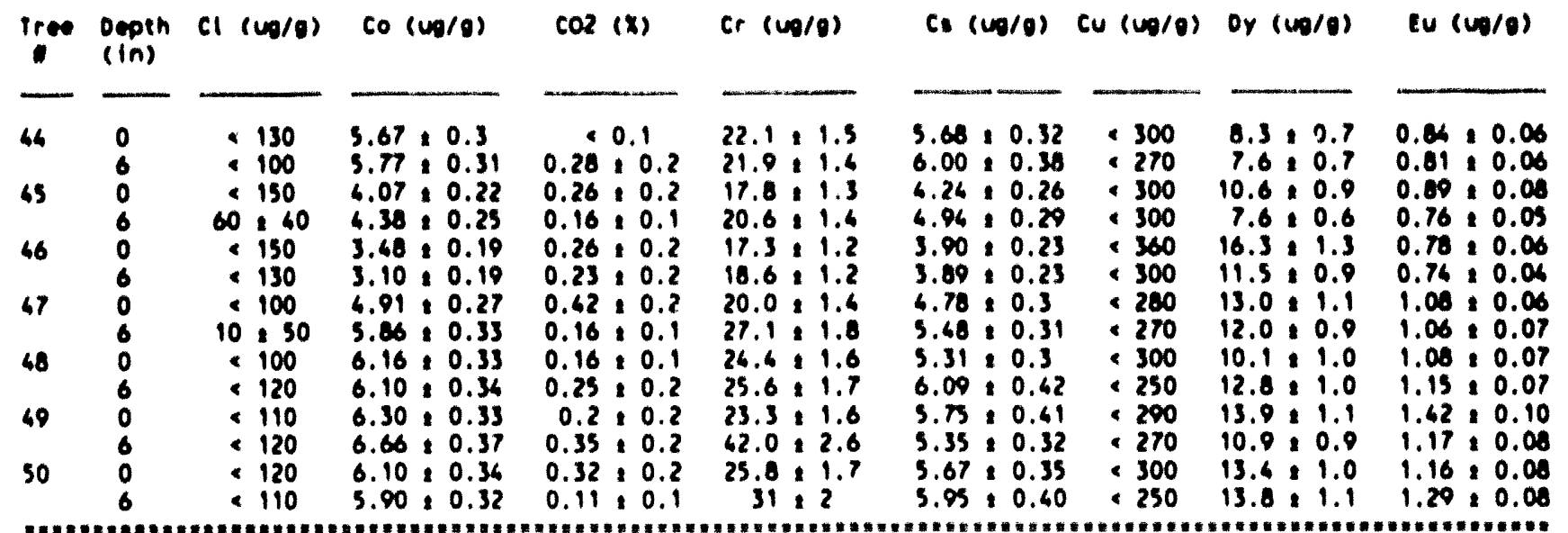

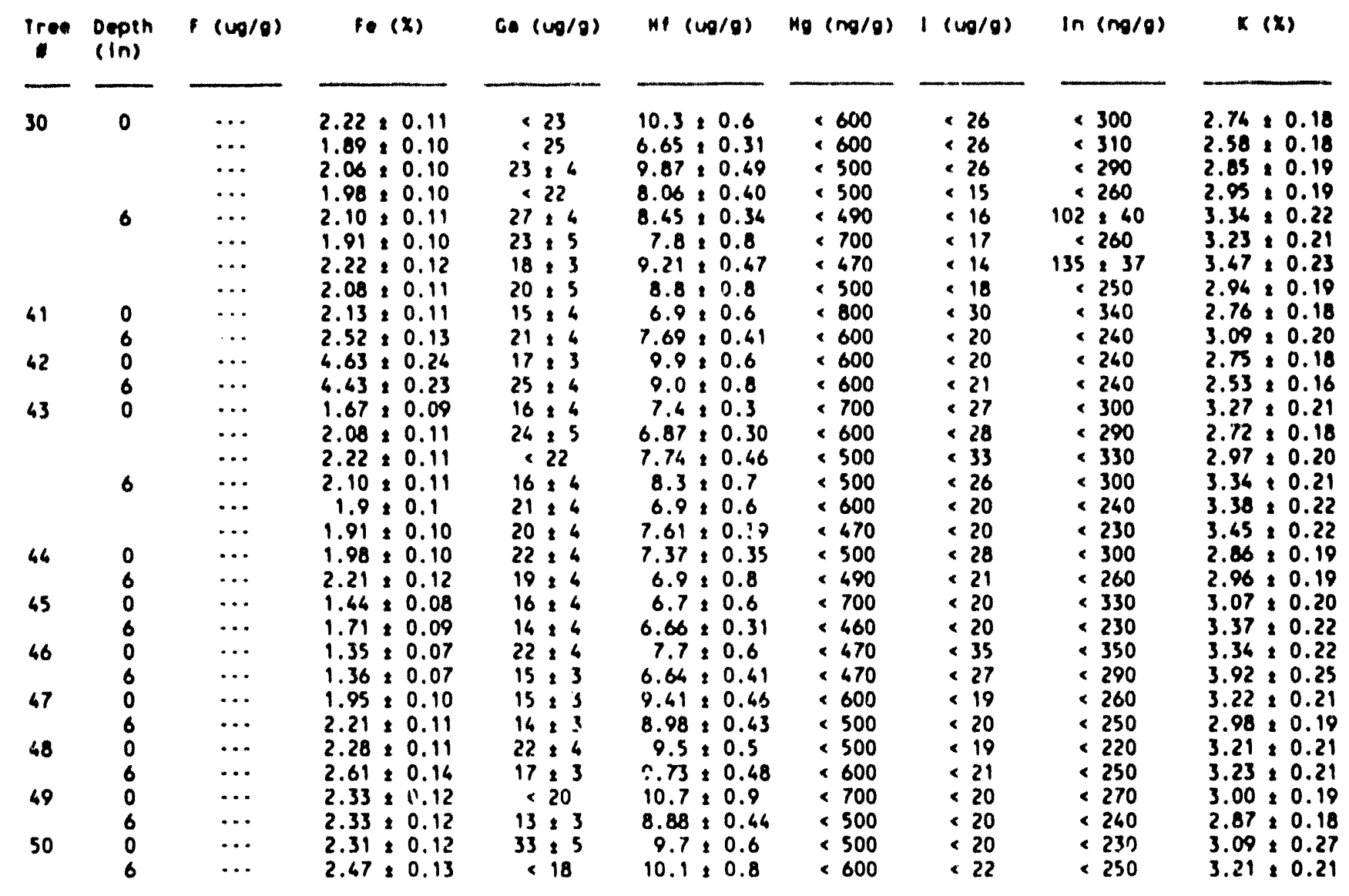




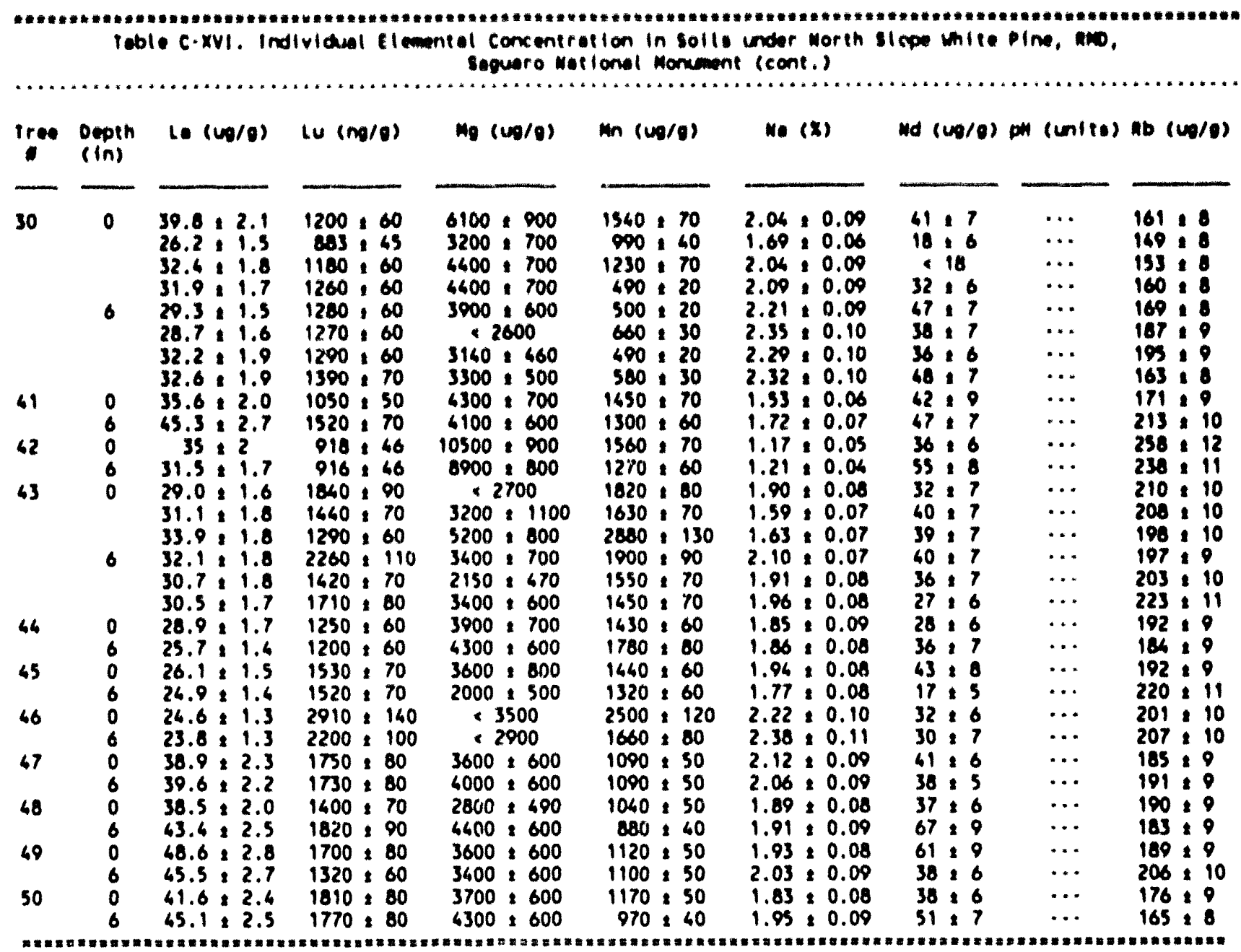

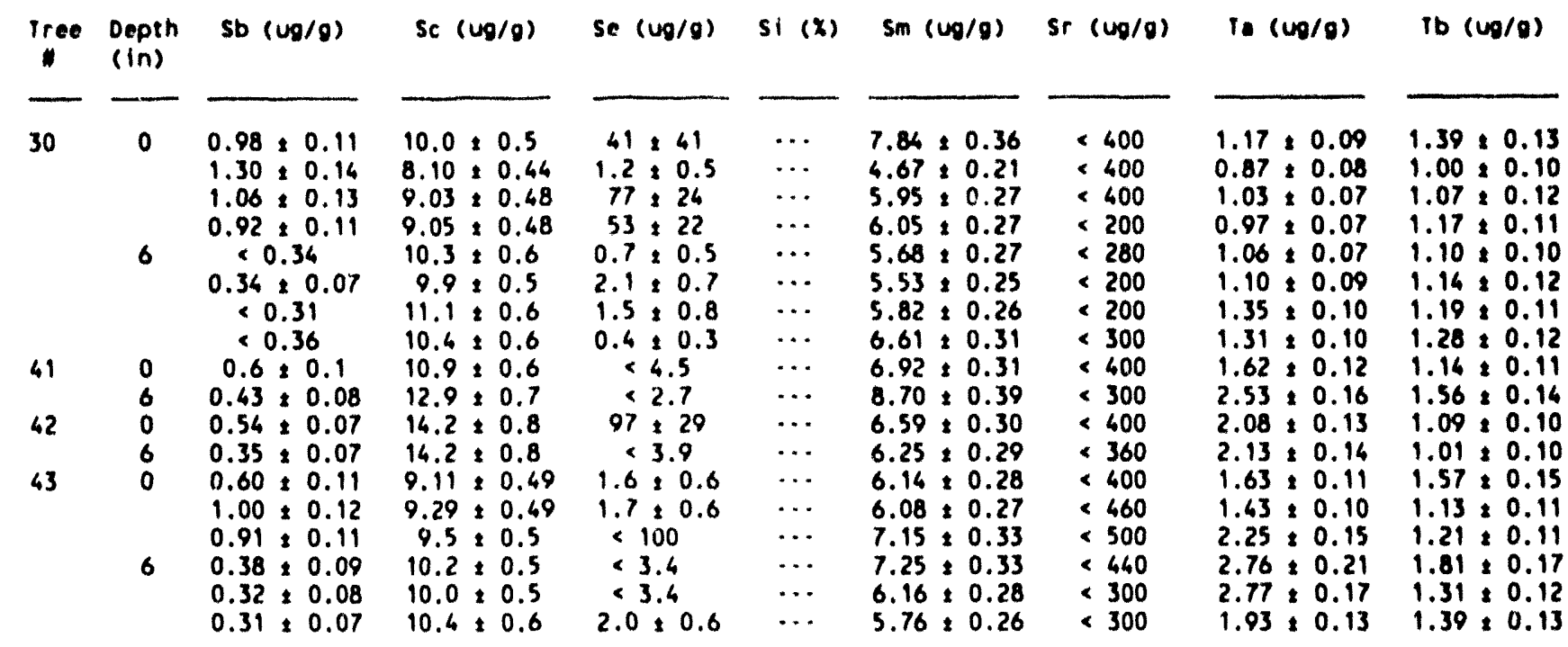




\begin{tabular}{|c|c|c|c|c|c|c|c|c|c|}
\hline iree & $\begin{array}{l}\text { Oepth } \\
\text { (In) }\end{array}$ & ib $(\omega / \theta)$ & se $(\omega / \theta)$ & se $(\omega \infty / \theta)$ & $81(3)$ & in $\left(w_{0} / g\right)$ & If $(\omega / \theta)$ & to $(u g / e)$ & ib $(\omega / \theta)$ \\
\hline $\begin{array}{l}46 \\
45 \\
46 \\
67 \\
48 \\
49 \\
50\end{array}$ & $\begin{array}{l}0 \\
6 \\
0 \\
6 \\
0 \\
0 \\
0 \\
6 \\
0 \\
6 \\
0 \\
6 \\
0 \\
6\end{array}$ & $\begin{array}{c}0.4: 0.10 \\
0.62: 0.08 \\
0.63: 0.10 \\
0.62: 0.10 \\
0.47: 0.10 \\
0.0 .33 \\
0.30: 0.13 \\
0.38: 0.09 \\
0.68: 0.12 \\
0.46: 0.09 \\
0.63: 0.12 \\
0.30: 0.09\end{array}$ & $\begin{array}{c:l}0.35: 0.5 \\
10.3: 0.5 \\
0.59: 0.66 \\
0.7: 0.5 \\
0.91: 0.67 \\
9.27: 0.69 \\
9.5: 0.5 \\
11.0: 0.6 \\
11.3: 0.6 \\
11.9: 0.6 \\
11.7: 0.6 \\
12.3: 0.7 \\
10.6: 0.6 \\
11.6: 0.6\end{array}$ & 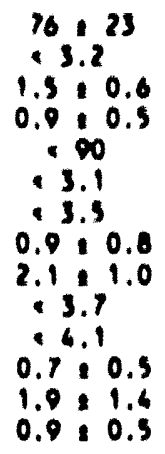 & $\begin{array}{l}\ldots \\
\ldots \\
\ldots \\
\ldots \\
\ldots \\
\ldots \\
\ldots \\
\ldots \\
\ldots \\
\ldots \\
\ldots\end{array}$ & $\begin{array}{l:l}5.63 & 0.23 \\
5.33 & 0.23 \\
5.69 & 0.26 \\
6.82 & 0.22 \\
3.09 & 0.28 \\
5.10 & 0.26 \\
8.09 & 0.37 \\
0.20 & 0.30 \\
7.67 & 0.35 \\
8.70 & 0.40 \\
10.0 & 0.5 \\
0.22 & 0.42 \\
8.48 & 0.30 \\
8.54 & 0.39\end{array}$ & $\begin{array}{l}400 \\
300 \\
300 \\
300 \\
-\mathbf{6 0 0} \\
460 \\
300 \\
300 \\
320 \\
3100 \\
300 \\
300 \\
320 \\
350\end{array}$ & $\begin{array}{l}1.00: 0.12 \\
2.37: 0.17 \\
2.16: 0.17 \\
2.18: 0.15 \\
1.09: 0.12 \\
2.05: 0.13 \\
1.69: 0.11 \\
2.04: 0.17 \\
1.61: 0.10 \\
1.79: 0.11 \\
1.66: 0.11 \\
1.73: 0.12 \\
1.61: 0.10 \\
1.61: 0.12\end{array}$ & $\begin{array}{l:l}1.16: 0.11 \\
0.00: 0.10 \\
1.30: 0.16 \\
1.16: 0.11 \\
1.91: 0.18 \\
1.53: 0.16 \\
1.70: 0.16 \\
1.55: 0.16 \\
1.64: 0.16 \\
1.62: 0.15 \\
1.07: 0.19 \\
1.30: 0.13 \\
1.76: 0.17 \\
1.73: 0.16\end{array}$ \\
\hline
\end{tabular}

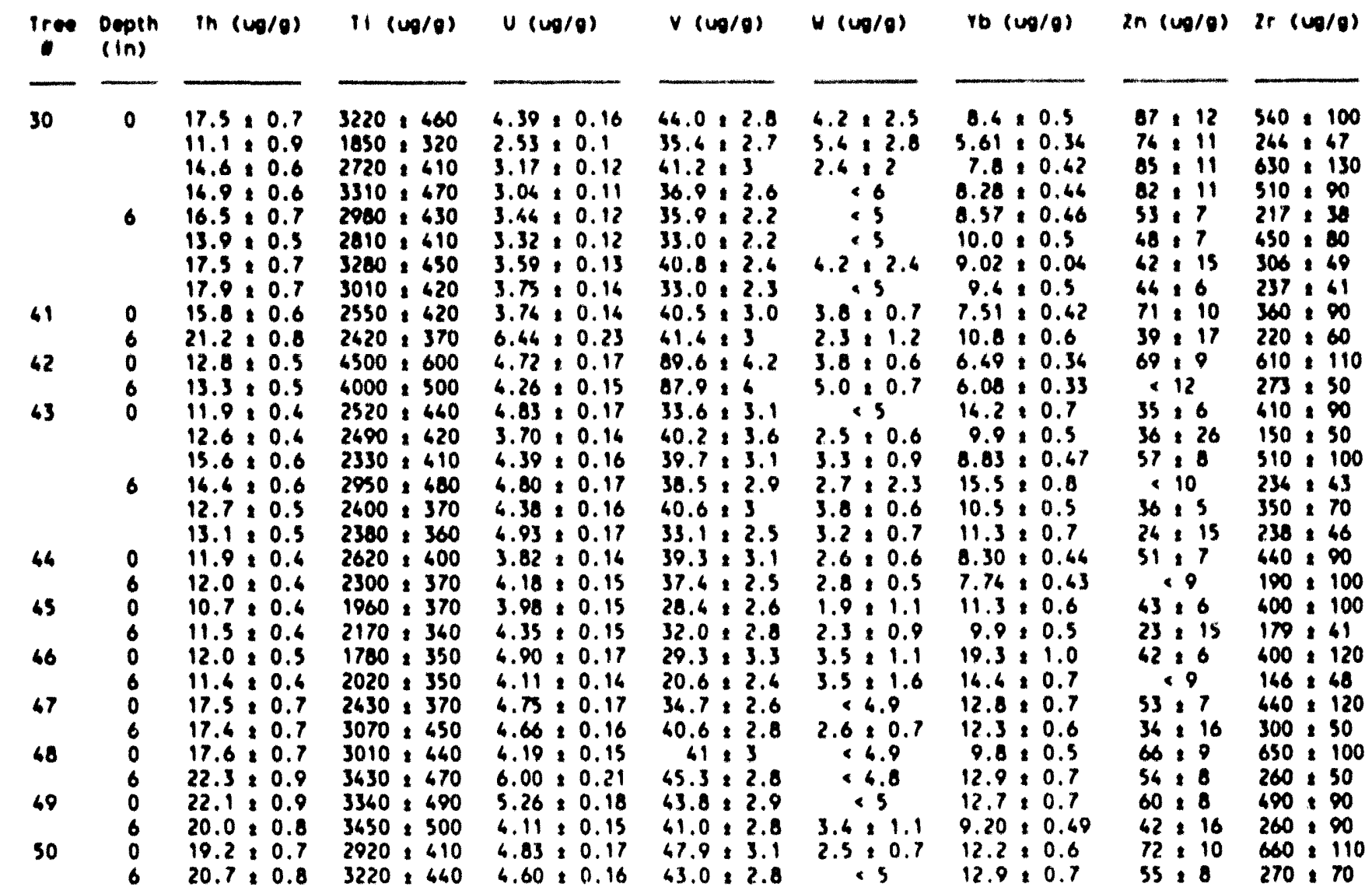




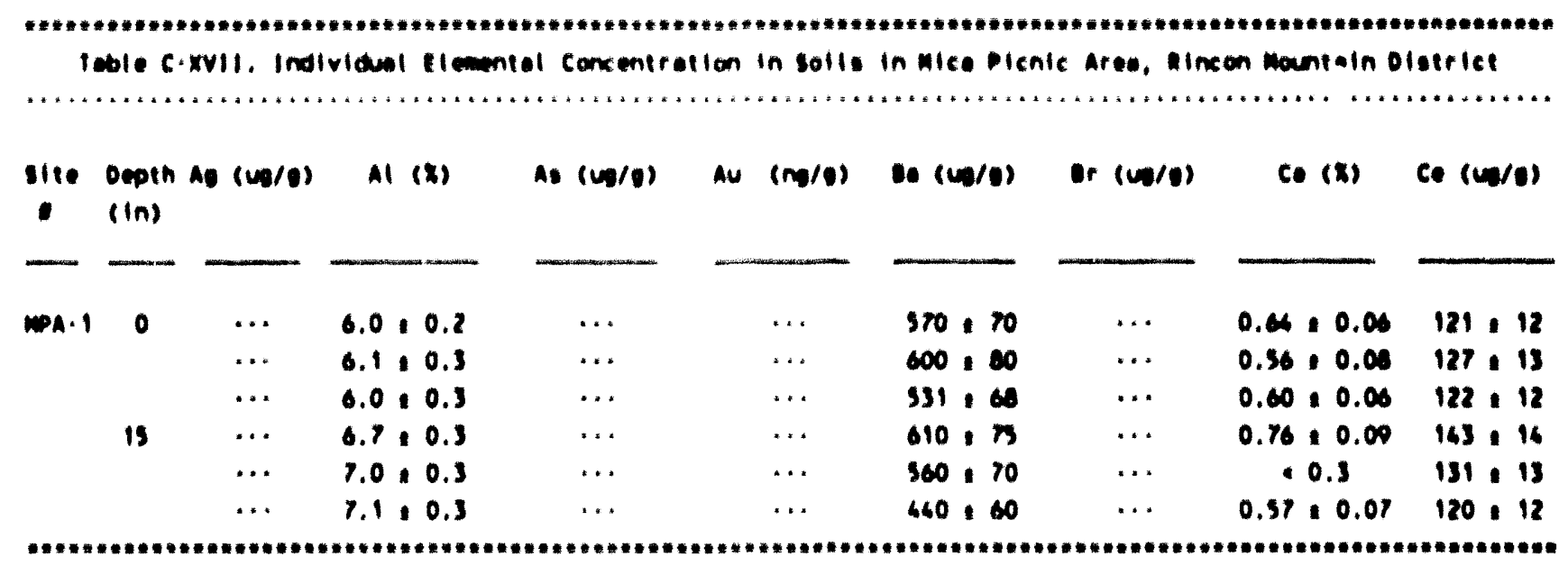

\begin{tabular}{|c|c|c|c|c|c|c|c|c|c|}
\hline sl1e & $\begin{array}{l}\text { oepth } \\
(\ln )\end{array}$ & Cl $(\omega / \theta)$ & $C_{0}\left(u_{0} / \theta\right)$ & $\cos (x)$ & $\operatorname{Cr}(\omega \infty / 0)$ & Co $(\omega) / g)$ & Cu $(\omega 0 / \theta)$ & Oy (ueles) & eu $(w / 0)$ \\
\hline \multirow[t]{6}{*}{ mPA. 1} & 0 & - 100 & $4.73: 0.40$ & $0.3: 0.2$ & $36.7: 2.63$ & $5.54: 0.58$ & $\cdots$ & $13.2 \cdot 1.1$ & $1.60: 0.17$ \\
\hline & & 140 & $5.20: 0.55$ & $0.6: 0.2$ & $35.8: 2.76$ & $5.75: 0.62$ & $\cdots$ & $19.0 \cdot 1.5$ & $1.73 * 0.18$ \\
\hline & & $10: 40$ & $5.84: 0.61$ & $0.4: 0.2$ & $46.5: 3.52$ & $5.65: 0.61$ & $\cdots$ & $15.2 \cdot 1.2$ & $1.67: 0.17$ \\
\hline & is & 100 & $6.17: 0.97$ & $0.3: 0.2$ & $39.8: 4.23$ & $7.1: 0.6$ & $\cdots$ & $13.3 \cdot 0.1$ & $1.73 \cdot 0.15$ \\
\hline & & $\cdot 120$ & $7.67 \cdot 1.21$ & $0.6: 0.2$ & $42.1: 6.31$ & $0.16: 0.76$ & $\cdots$ & $16.7 \cdot 1.3$ & $1.7 \mathrm{*}: 0.16$ \\
\hline & & 150 & $0.26: 1.3$ & $0.5: 0.2$ & $40.8: 6.46$ & $0.21: 0.73$ & $\cdots$ & $15.3 \cdot 1.3$ & $1.56: 0.16$ \\
\hline
\end{tabular}

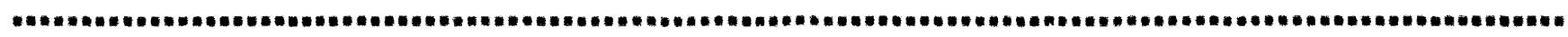

\begin{tabular}{|c|c|c|c|c|c|c|c|c|c|}
\hline site & $\begin{array}{l}\text { Depth } \\
\text { (in) }\end{array}$ & $1(100 / 0)$ & $f e(x)$ & Ga $(\log / \theta)$ & NI $(\log / \theta)$ & Ho $(\omega 0 / \theta)$ & $1(00 / 0)$ & $\ln (\omega 0 / g)$ & $x(x)$ \\
\hline \multirow[t]{6}{*}{$M P A=1$} & 0 & $\cdots$ & $3.06: 0.21$ & 430 & $17.7: 1.6$ & $\ldots$ & $\cdots$ & 0.10 & $3.1 \cdot 0.5$ \\
\hline & & $\cdots$ & $3.08: 0.21$ & c 50 & $16.7: 1.3$ & $\cdots$ & $\cdots$ & c 0.10 & $2.7: 0.3$ \\
\hline & & $\cdots$ & $4.23: 0.30$ & .30 & $22.9 \cdot 1.8$ & $\cdots$ & $\cdots$ & 0.11 & $2.8: 0.6$ \\
\hline & 15 & $\cdots$ & $3.87: 0.31$ & .60 & $16.6: 1.3$ & $\cdots$ & $\cdots$ & 0.16 & $3.0: 0.2$ \\
\hline & & $\cdots$ & $3.83: 0.31$ & - 50 & $17.1: 1.4$ & $\cdots$ & $\cdots$ & 0.16 & $2.6: 0.2$ \\
\hline & & $\cdots$ & $3.59=0.29$ & $<50$ & $16.5: 1.3$ & $\cdots$ & $\cdots$ & 0.13 & $2.0: 0.3$ \\
\hline
\end{tabular}

Note: Ihis orea has many dead and dying waguaro cacti 


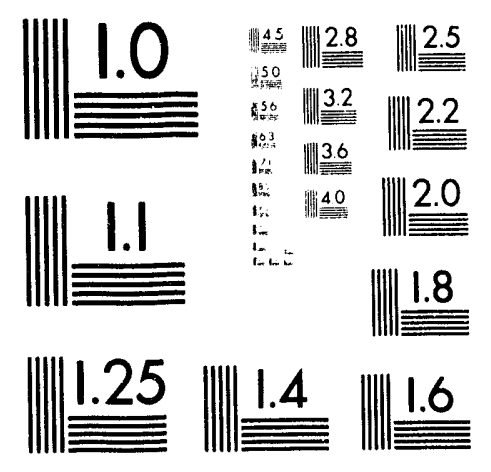



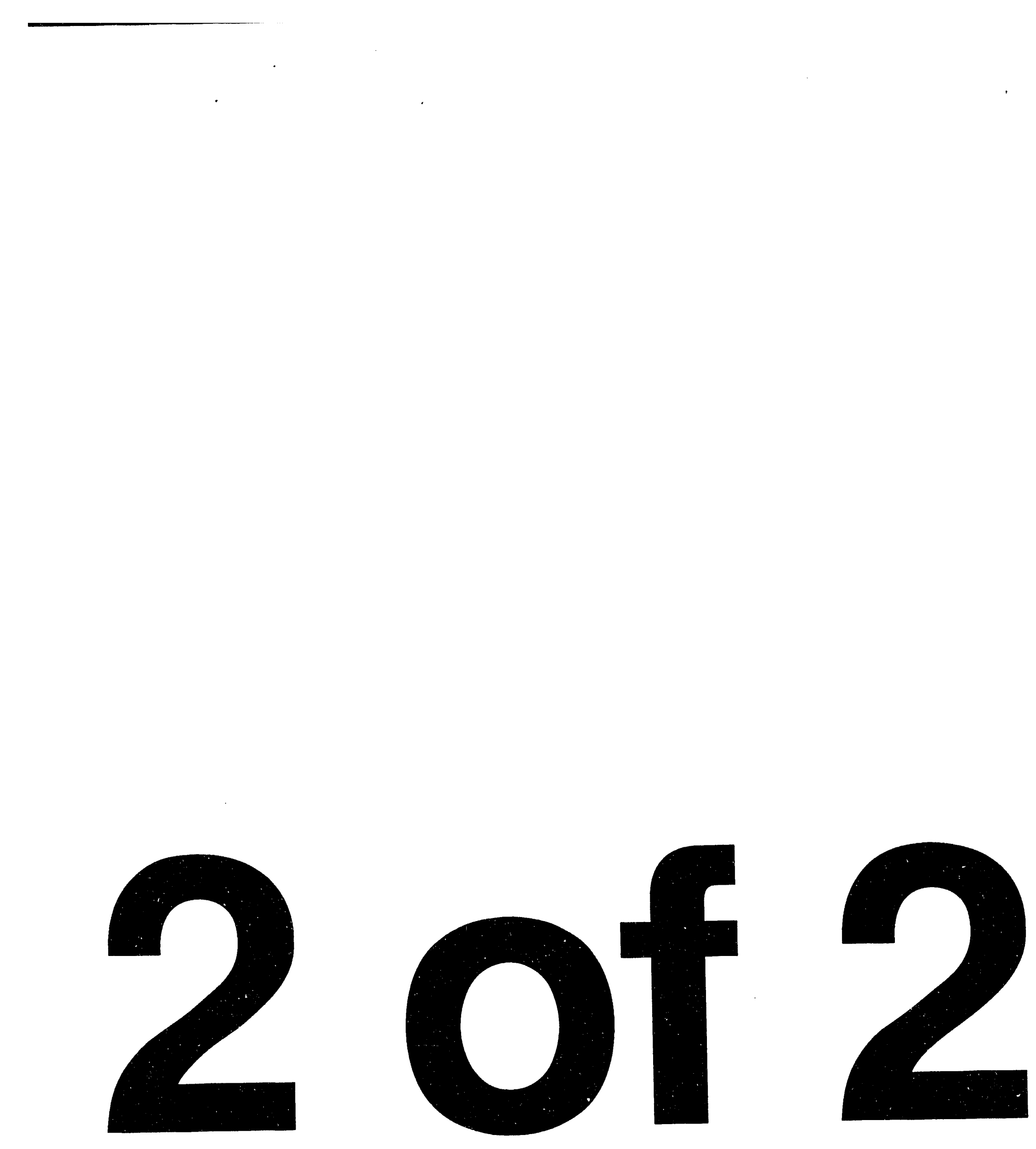


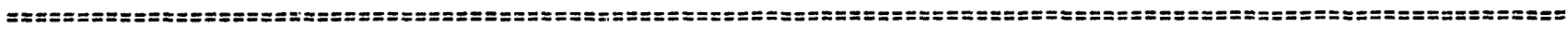

Table C-XVIl. Individual Elemental Concentration in Soils in Mica Picnic Area, Rincon Mountain District (cont.)

\begin{tabular}{|c|c|c|c|c|c|c|c|c|c|}
\hline $\begin{array}{c}\text { Site } \\
\end{array}$ & $\begin{array}{l}\text { Depth } \\
\text { (in) }\end{array}$ & La (ug/g) & Lu (ug/g) & $M g(n g / g)$ & Mn $(u g / g)$ & $\mathrm{Na}(x)$ & Hd $(u g / g)$ & pH (units) & $R b(u g / g)$ \\
\hline \multirow[t]{6}{*}{ MPA-1 } & 0 & $\ldots$ & $\ldots$ & $<2000$ & $720 \pm 30$ & $1.41 \pm 0.05$ & $\cdots$ & $\cdots$ & $150 \pm 17$ \\
\hline & & $\cdots$ & $\cdots$ & $<4000$ & $880 \pm 40$ & $1.37 \pm 0.05$ & $\cdots$ & $\cdots$ & $156 \pm 17$ \\
\hline & & $\ldots$ & $\cdots$ & $<2000$ & $880 \pm 40$ & $1.38 \pm 0.05$ & $\cdots$ & $\cdots$ & $143 \pm 16$ \\
\hline & 15 & $\ldots$ & $\ldots$ & $4800 \pm 1300$ & $1060 \pm 50$ & $1.32 \pm 0.05$ & $\cdots$ & $\ldots$ & $137 \pm 15$ \\
\hline & & $\cdots$ & $\cdots$ & $<3000$ & $910 \pm 40$ & $1.13 \pm 0.05$ & $\cdots$ & $\cdots$ & $137 \pm 15$ \\
\hline & & $\ldots$ & $\cdots$ & $<3000$ & $1040 \pm 50$ & $1.21 \pm 0.04$ & $\cdots$ & $\cdots$ & $146 \pm 16$ \\
\hline
\end{tabular}

\begin{tabular}{|c|c|c|c|c|c|c|c|c|c|}
\hline $\begin{array}{c}\text { Site } \\
\#\end{array}$ & $\begin{array}{l}\text { Depth } \\
\text { (in) }\end{array}$ & Sb (ug/g) & Sc $(u g / g)$ & Se (ug/g) & Si $(\%)$ & $\operatorname{Sm}(u g / g)$ & $\operatorname{Sr}(u g / g)$ & Ta $(u g / g)$ & Tb $(u g / g)$ \\
\hline \multirow[t]{6}{*}{ MPA-1 } & 0 & $1.41 \pm 0.20$ & $7.94 \pm 0.40$ & $\cdots$ & $34.6 \pm 1.6$ & $13.3 \pm 1.3$ & $<190$ & $1.23 \pm 0.14$ & $\cdots$ \\
\hline & & $1.47 \pm 0.22$ & $7.85 \pm 0.40$ & $\cdots$ & $32 \pm 2$ & $14.0 \pm 1.4$ & $<300$ & $1.19 \pm 0.15$ & $\cdots$ \\
\hline & & $1.54 \pm 0.22$ & $8.99 \pm 0.46$ & $\cdots$ & $33 \pm 2$ & $13.3 \pm 1.3$ & $<200$ & $1.76 \pm 0.20$ & $\cdots$ \\
\hline & 15 & $1.67 \pm 0.31$ & $9.39 \pm 0.66$ & $\cdots$ & $31 \pm 2$ & $14.2 \pm 2.1$ & $<300$ & $1.64 \pm 0.27$ & $\cdots$ \\
\hline & & $1.37 \pm 0.29$ & $11.1 \pm 0.78$ & $\ldots$ & $31 \pm 2$ & $13.0 \pm 1.9$ & $<200$ & $1.41 \pm 0.25$ & $\ldots$ \\
\hline & & $1.18 \pm 0.27$ & $9.54 \pm 0.67$ & $\cdots$ & $30 \pm 2$ & $10.3 \pm 1.9$ & $<300$ & $8.86 \pm 1.35$ & $\cdots$ \\
\hline
\end{tabular}

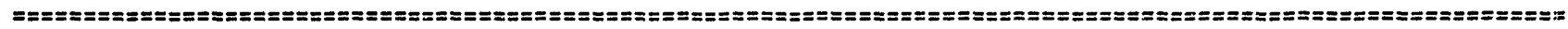

\begin{tabular}{|c|c|c|c|c|c|c|c|c|c|}
\hline $\begin{array}{c}\text { Site } \\
*\end{array}$ & $\begin{array}{l}\text { Depth } \\
\text { (in) }\end{array}$ & Th $(u g / g)$ & $\mathrm{Ti}(u g / g)$ & $U(u g / g)$ & $V(u g / g)$ & $W(u g / g)$ & $\mathrm{Yb}(u g / g)$ & $\ln (u g / g)$ & $2 r(u g / g)$ \\
\hline \multirow[t]{6}{*}{$M P A-1$} & 0 & $25.4 \pm 2.0$ & $4000 \pm 600$ & $3.78 \pm 0.3$ & $59 \pm 3$ & $<110$ & $11.0 \pm 1.1$ & $66 \pm 10$ & $\cdots$ \\
\hline & & $24.7 \pm 2.0$ & $3700 \pm 300$ & $4.33 \pm 0.4$ & $57 \pm 4$ & $<140$ & $11.5 \pm 1.1$ & $63 \pm 10$ & $\cdots$ \\
\hline & & $23.1+1.8$ & $5200 \pm 700$ & $4.99 \pm 0.4$ & $76 \pm 4$ & $<110$ & $15.5 \pm 1.5$ & $63 \pm 10$ & $\ldots$ \\
\hline & 15 & $25.6 \pm 2.0$ & $4600 \pm 400$ & $3.75 \pm 0.3$ & $61 \pm 4$ & $<130$ & $10.6 \pm 1.0$ & $68 \pm 14$ & $\cdots$ \\
\hline & & $22.9 \pm 1.8$ & $4800 \pm 400$ & $3.72 \pm 0.3$ & $61 \pm 3$ & $<140$ & $10.2 \pm 1.0$ & $82 \pm 16$ & $\cdots$ \\
\hline & & $20.6 \pm 1.6$ & $4300 \pm 300$ & $3.86 \pm 0.3$ & $59 \pm 3$ & $<120$ & $9.42 \pm 0.97$ & $84 \pm 17$ & $\cdots$ \\
\hline
\end{tabular}

Note: This is an area of many dead and dying saguaro cact $i$ 


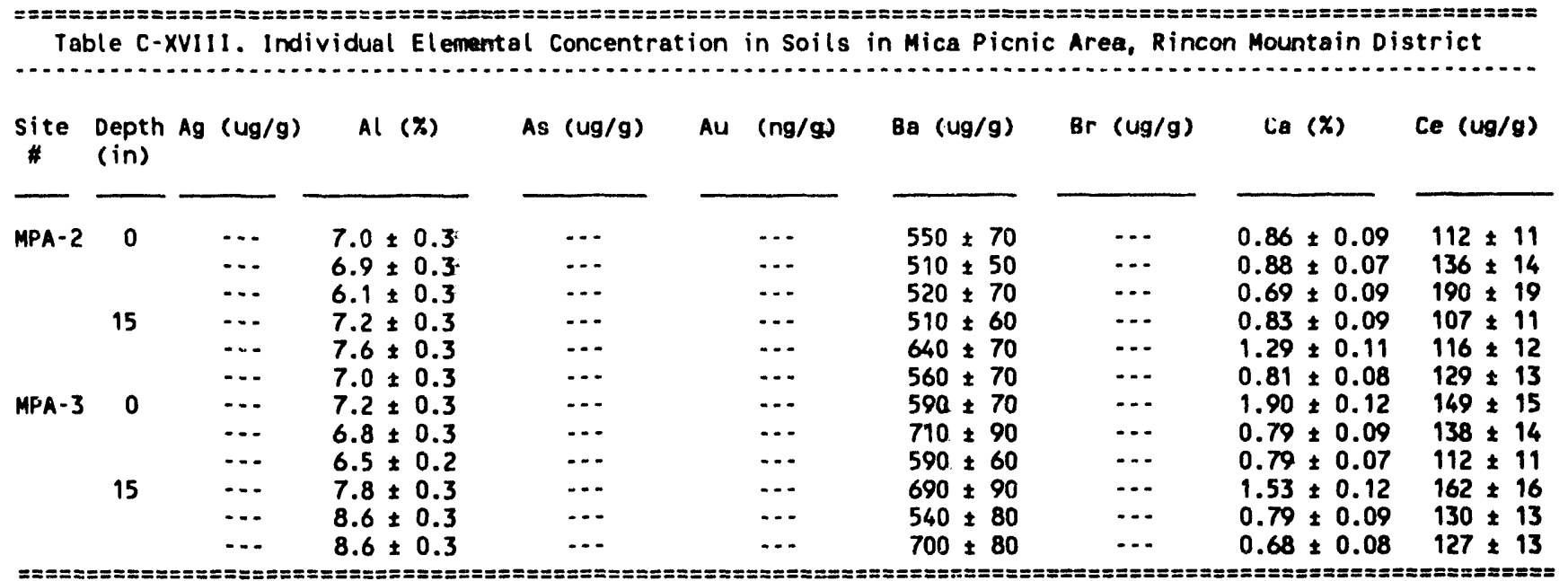

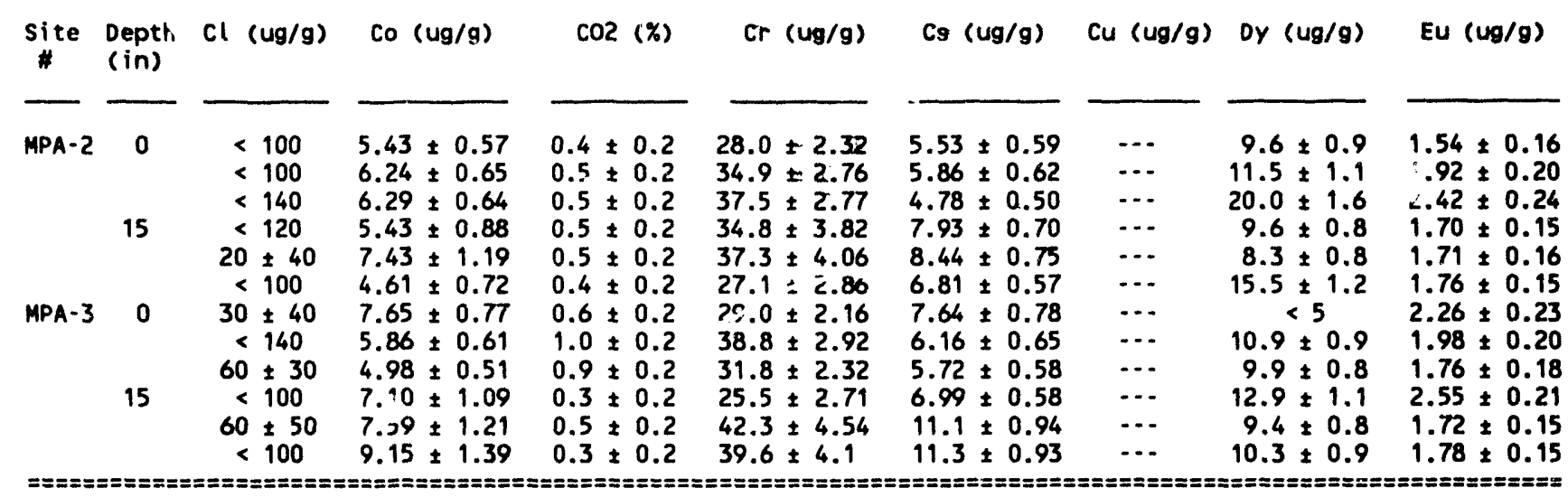

\begin{tabular}{|c|c|c|c|c|c|c|c|c|c|}
\hline $\begin{array}{c}\text { site } \\
\#\end{array}$ & $\begin{array}{l}\text { Depth } \\
\text { (in) }\end{array}$ & $F(u g / g)$ & $\mathrm{Fe}(x)$ & $\mathrm{Ga}(u g / g)$ & $H f(u g / g)$ & $\mathrm{Hg}(u g / g)$ & $1(u g / g)$ & $\ln (u g / g)$ & $K(x)$ \\
\hline \multirow[t]{6}{*}{ MPA-2 } & 0 & $\ldots$ & $2.32 \pm 0.16$ & $<40$ & $8.45 \pm 0.69$ & $\ldots$ & $\ldots$ & $<0.17$ & $2.7 \pm 0.2$ \\
\hline & & $\ldots$ & $2.8 \pm 0.2$ & $<30$ & $10.5 \pm 0.86$ & $\ldots$ & $\ldots$ & $<0.11$ & $2.7 \pm 0.3$ \\
\hline & & $\ldots$ & $5.67 \pm 0.40$ & $<50$ & $31.7 \pm 2.56$ & $\cdots$ & $\cdots$ & $<0.19$ & $3.0 \pm 0.3$ \\
\hline & 15 & $\cdots$ & $2.50 \pm 0.20$ & $<50$ & $10.5 \pm 0.86$ & $\cdots$ & $\cdots$ & $<0.14$ & $2.6 \pm 0.2$ \\
\hline & & $\cdots$ & $3.17 \pm 0.25$ & $<50$ & $9.13 \pm 0.76$ & $\cdots$ & $\cdots$ & $<0.12$ & $2.7 \pm 0.2$ \\
\hline & & $\ldots$ & $2.76 \pm 0.22$ & $<50$ & $11.3 \pm 0.91$ & $\cdots$ & $\ldots$ & $<0.15$ & $c .7 \pm 0.2$ \\
\hline \multirow[t]{6}{*}{ MPA-3 } & 0 & $\cdots$ & $3.12 \pm 0.22$ & $<30$ & $11.4 \pm 0.92$ & $\cdots$ & $\cdots$ & $<0.11$ & $2.8 \pm 0.3$ \\
\hline & & $\ldots$ & $3.05 \pm 0.21$ & $<50$ & $16.8 \pm 1.36$ & $\cdots$ & $\cdots$ & $<0.17$ & $3.0 \pm 0.2$ \\
\hline & & $\cdots$ & $2.51 \pm 0.17$ & $<30$ & $13.0 \pm 1.06$ & $\cdots$ & $\cdots$ & $<0.10$ & $2.7 \pm 0.3$ \\
\hline & 15 & $\ldots$ & $3.61 \pm 0.29$ & $<5 i$ & $13.5 \pm 1.09$ & $\cdots$ & $\cdots$ & $<0.13$ & $2.6 \pm 0.2$ \\
\hline & & $\cdots$ & $3.32 \pm 0.26$ & $<50$ & $12.2 \pm 1.01$ & $\cdots$ & $\cdots$ & $<0.16$ & $2.6 \pm 0.2$ \\
\hline & & & $3.21 \pm 0.26$ & $=50$ & $11.5 \pm 0.93$ & $\cdots$ & $\cdots$ & $<0.13$ & $2.9 \pm 0.2$ \\
\hline
\end{tabular}

Note: These wash sites contain many heal thy saguaro cact $i$ 


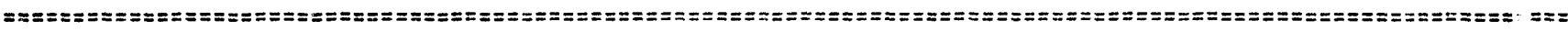
Table C-XVIII. Individual Elemental Concentration in Soils in Mica Picnic Area, Rincon Mountain District (cont.)

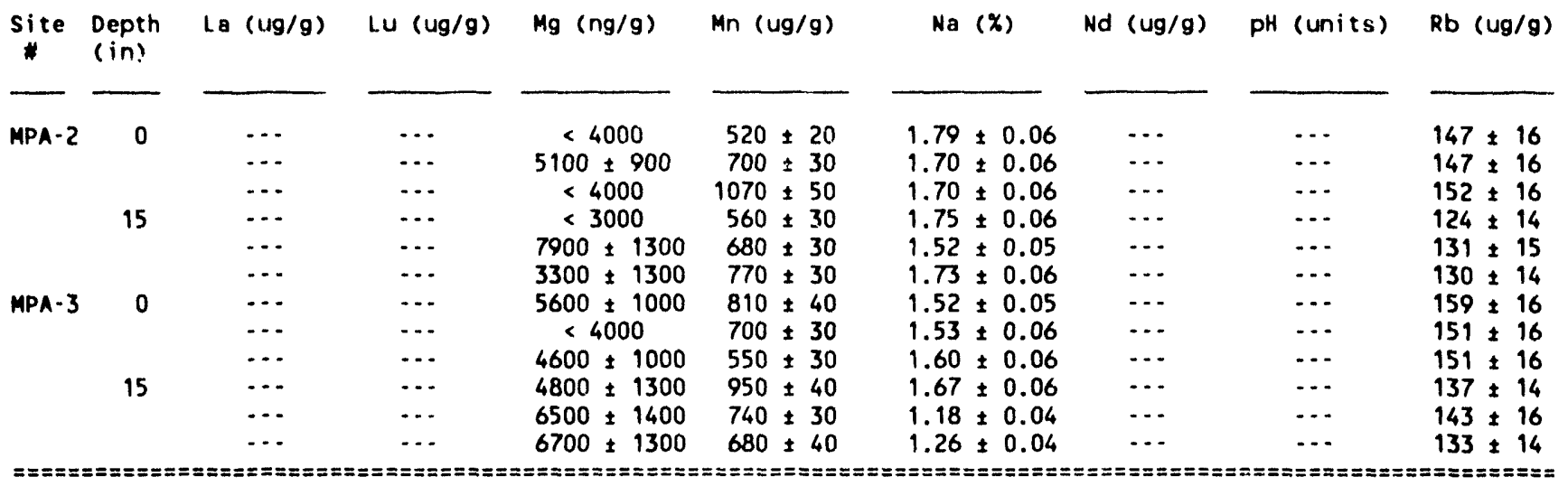

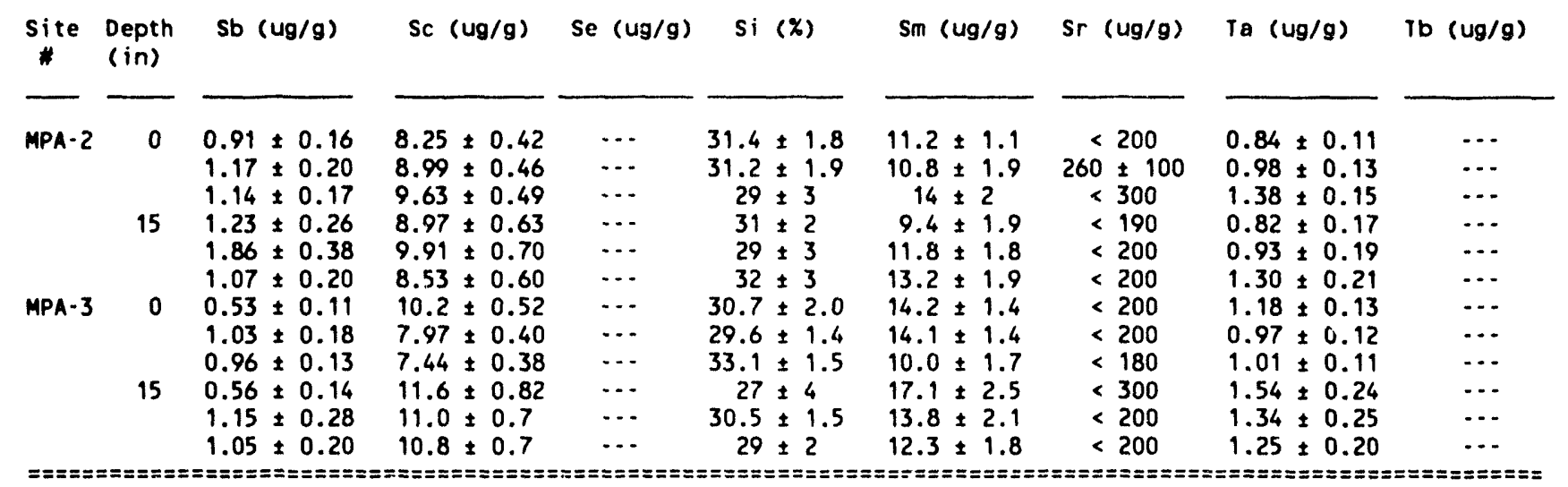

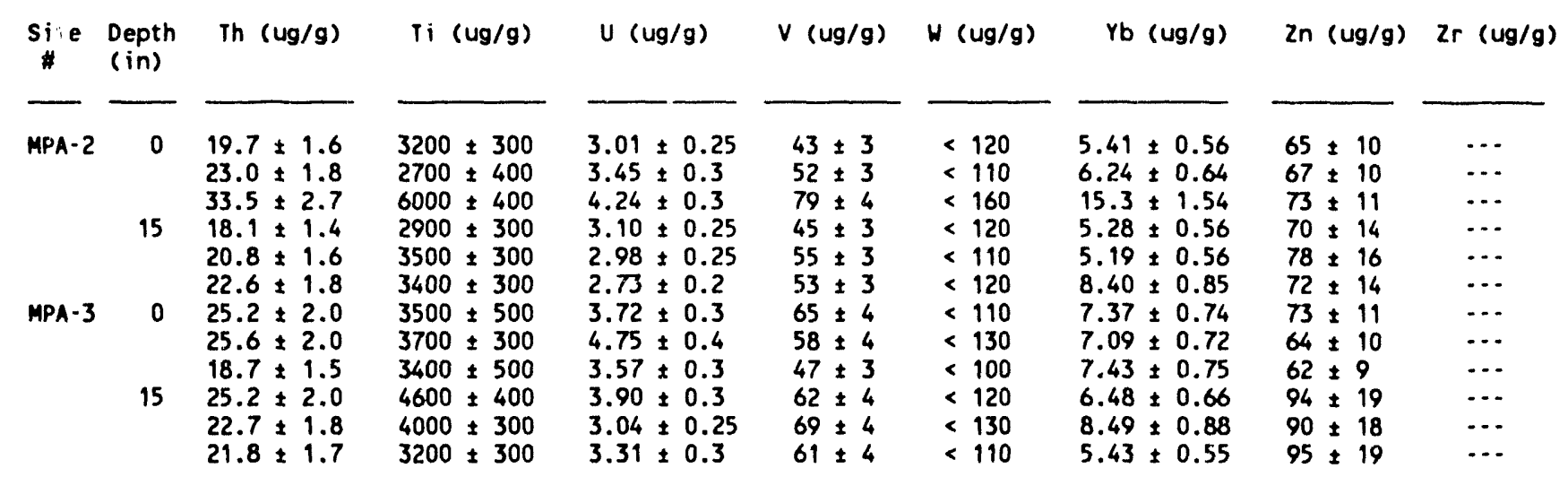

Note: These wash sites contain many heal thy saguaro cacti 


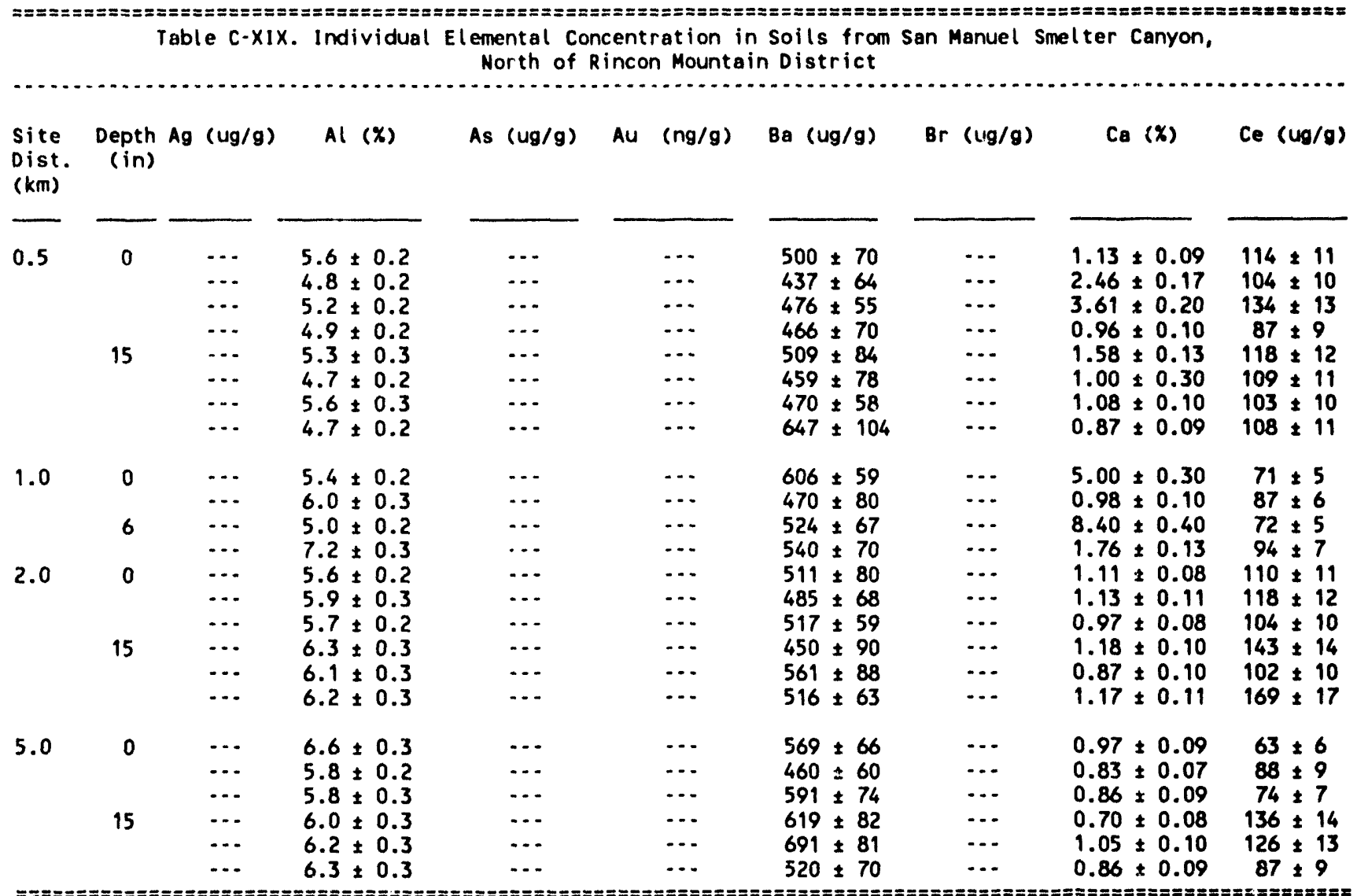

\begin{tabular}{|c|c|c|c|c|c|c|c|c|c|}
\hline $\begin{array}{l}\text { Site } \\
\text { Dist. } \\
\text { (km) }\end{array}$ & $\begin{array}{l}\text { Depth } \\
\text { (in) }\end{array}$ & $\mathrm{Cl}(u g / g)$ & Co (ug/g) & $\cos (x)$ & $\operatorname{Cr}(u g / g)$ & Cs $(u g / g)$ C & $\mathrm{Cu}(u g / g)$ & Dy (ug/g) & Eu (ug/g) \\
\hline 0.5 & 15 & $\begin{aligned} 50 & \pm 40 \\
< & 140 \\
10 & \pm 40 \\
< & 150 \\
< & 100 \\
50 & \pm 50 \\
< & 100 \\
20 & \pm 50\end{aligned}$ & $\begin{array}{l}17.7 \pm 1.8 \\
16.7 \pm 1.69 \\
14.4 \pm 1.45 \\
14.2 \pm 1.45 \\
17.7 \pm 2.7 \\
22.3 \pm 3.37 \\
14.1 \pm 2.13 \\
15.4 \pm 2.36\end{array}$ & $\begin{array}{l}0.3 \pm 0.2 \\
1.9 \pm 0.2 \\
3.2 \pm 0.2 \\
0.6 \pm 0.2 \\
0.5 \pm 0.2 \\
0.4 \pm 0.2 \\
0.6 \pm 0.2 \\
0.2 \pm 0.2\end{array}$ & $\begin{array}{l}79.6 \pm 5.91 \\
71.8 \pm 5.25 \\
57.5 \pm 4.15 \\
63.9 \pm 4.77 \\
66.6 \pm 7.07 \\
81.9 \pm 8.45 \\
68.4 \pm 6.97 \\
66.3 \pm 7.03\end{array}$ & $\begin{array}{l}8.92 \pm 0.96 \\
7.07 \pm 0.76 \\
6.80 \pm 0.70 \\
7.23 \pm 0.79 \\
8.44 \pm 0.79 \\
8.38 \pm 0.80 \\
9.34 \pm 0.78 \\
7.99 \pm 0.75\end{array}$ & $\begin{array}{l}\cdots \\
\cdots \\
\cdots \\
\cdots \\
\cdots \\
\cdots \\
\cdots \\
\cdots\end{array}$ & $\begin{array}{c}9.20 \pm 0.8 \\
10.0 \pm 0.9 \\
\leq 6 \\
10.9 \pm 1.0 \\
13.0 \pm 1.1 \\
10.4 \pm 1.0 \\
10.7 \pm 1.0 \\
12.3 \pm 1.0\end{array}$ & $\begin{array}{l}1.76 \pm 0.19 \\
1.65 \pm 0.18 \\
1.55 \pm 0.16 \\
1.73 \pm 0.18 \\
1.63 \pm 0.15 \\
1.66 \pm 0.15 \\
1.58 \pm 0.13 \\
1.98 \pm 0.18\end{array}$ \\
\hline 1.0 & $\begin{array}{l}0 \\
6\end{array}$ & $\begin{array}{r}120 \pm 30 \\
70 \pm 30 \\
100 \pm 20 \\
110 \pm 30\end{array}$ & $\begin{array}{l}12.0 \pm 1.23 \\
18.9 \pm 1.92 \\
13.9 \pm 1.47 \\
19.7 \pm 2.04\end{array}$ & $\begin{array}{l}5.6 \pm 0.6 \\
0.8 \pm 0.2 \\
9.9 \pm 1.0 \\
1.3 \pm 0.2\end{array}$ & $\begin{array}{l}44 \pm 2 \\
63 \pm 3 \\
48 \pm 3 \\
59 \pm 3\end{array}$ & $\begin{array}{l}6.55 \pm 0.51 \\
7.51 \pm 0.59 \\
6.87 \pm 0.57 \\
8.50 \pm 0.73\end{array}$ & $\begin{array}{l}\cdots \\
\cdots \\
\cdots \\
\cdots\end{array}$ & $\begin{array}{r}6.9 \pm 0.6 \\
7.4 \pm 0.7 \\
10.9 \pm 1.0 \\
9.4 \pm 0.8\end{array}$ & $\begin{array}{l}1.34 \pm 0.10 \\
1.61 \pm 0.12 \\
1.48 \pm 0.12 \\
1.62 \pm 0.13\end{array}$ \\
\hline 2.0 & 15 & $\begin{aligned}< & 110 \\
< & 150 \\
90 & \pm 40 \\
10 & \pm 40 \\
< & 140 \\
110 & \pm 70\end{aligned}$ & $\begin{array}{l}16.1 \pm 1.64 \\
13.8 \pm 1.41 \\
15.4 \pm 1.55 \\
14.5 \pm 2.22 \\
16.5 \pm 2.52 \\
14.7 \pm 2.22\end{array}$ & $\begin{array}{l}0.6 \pm 0.2 \\
0.4 \pm 0.2 \\
0.4 \pm 0.2 \\
0.3 \pm 0.2 \\
0.3 \pm 0.2 \\
0.2 \pm 0.2\end{array}$ & $\begin{array}{l}59.8 \pm 4.52 \\
54.5 \pm 4.17 \\
58.4 \pm 4.22 \\
57.8 \pm 6.19 \\
46.2 \pm 5.1 \\
56.6 \pm 5.78\end{array}$ & $\begin{aligned} 8.45 & \pm 0.89 \\
8.6 & \pm 0.92 \\
8.48 & \pm 0.87 \\
10.2 & \pm 0.91 \\
9.85 & \pm 0.89 \\
9.08 & \pm 0.76\end{aligned}$ & $\begin{array}{l}\ldots \\
\cdots \\
\cdots \\
\cdots \\
\cdots \\
\cdots\end{array}$ & $\begin{array}{c}<6 \\
9.0 \pm 0.9 \\
<5 \\
12.8 \pm 1.1 \\
14.2 \pm 1.2 \\
9.3 \pm 0.8\end{array}$ & $\begin{array}{l}1.78 \pm 0.19 \\
1.68 \pm 0.18 \\
1.75 \pm 0.18 \\
1.97 \pm 0.18 \\
1.72 \pm 0.16 \\
1.90 \pm 0.16\end{array}$ \\
\hline 5.0 & 15 & $\begin{aligned}< & 130 \\
30 & \pm 30 \\
< & 140 \\
80 & \pm 50 \\
20 & \pm 40 \\
< & 110\end{aligned}$ & $\begin{array}{l}9.58 \pm 0.97 \\
11.8 \pm 1.19 \\
11.0 \pm 1.13 \\
16.0 \pm 2.43 \\
16.0 \pm 2.41 \\
15.0 \pm 2.29\end{array}$ & $\begin{array}{l}0.4 \pm 0.2 \\
0.9 \pm 0.2 \\
0.5 \pm 0.2 \\
0.3 \pm 0.2 \\
0.4 \pm 0.2 \\
0.3 \pm 0.2\end{array}$ & $\begin{array}{l}33.3 \pm 2.5 \\
65.0 \pm 4.6 \\
47.0 \pm 3.5 \\
64.9 \pm 6.7 \\
61.9 \pm 6.3 \\
64.9 \pm 6.7\end{array}$ & $\begin{array}{l}7.01 \pm 0.73 \\
7.25 \pm 0.74 \\
8.04 \pm 0.86 \\
8.39 \pm 0.75 \\
9.61 \pm 0.8 \\
9.43 \pm 0.86\end{array}$ & $\begin{array}{l}\cdots \\
\cdots \\
\cdots \\
\cdots \\
\cdots \\
\cdots\end{array}$ & $\begin{aligned} 4.7 & \pm 0.4 \\
7.1 & \pm 0.6 \\
6.6 & \pm 0.6 \\
7.1 & \pm 0.6 \\
10 & \pm 2 \\
10.6 & \pm 0.9\end{aligned}$ & $\begin{array}{l}1.46 \div 0.15 \\
1.87: 0.19 \\
1.54: 0.17 \\
1.85 \pm 0.16 \\
1.79 \neq 0.15 \\
1.70 \div 0.15\end{array}$ \\
\hline
\end{tabular}




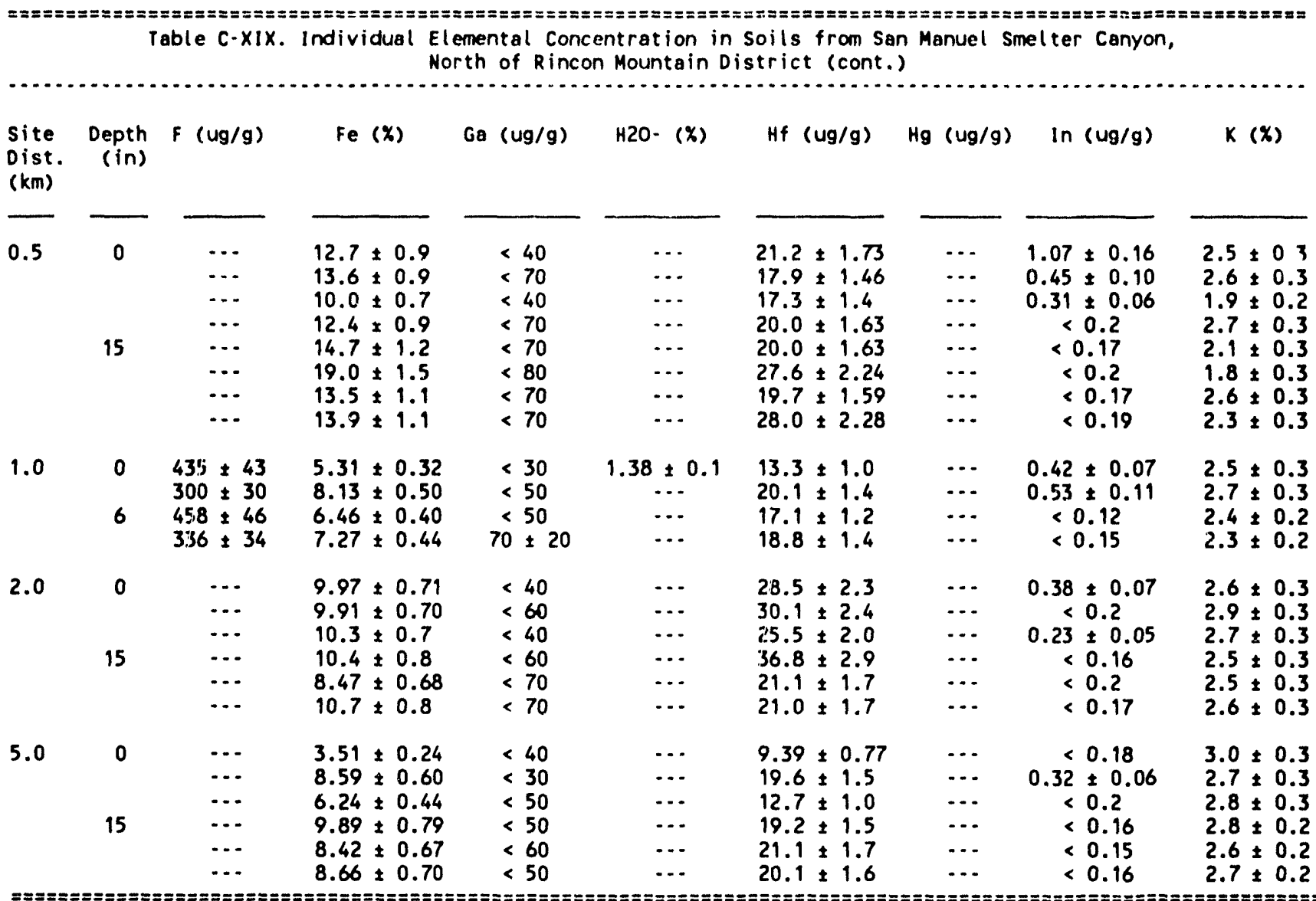

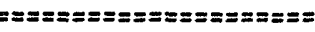

\begin{tabular}{|c|c|c|c|c|c|}
\hline $\begin{array}{l}\text { Site } \\
\text { Dist. } \\
\text { (km) }\end{array}$ & $\begin{array}{r}\text { Depth } \\
\text { (in) }\end{array}$ & La (ug/g) & $\operatorname{Lu}(u g / g)$ & $M g$ (ug/g) & $\operatorname{Mn}(u g / g)$ \\
\hline 0.5 & 0 & $\begin{array}{l}\cdots \\
\cdots \\
\cdots \\
\cdots \\
\cdots \\
\cdots \\
\cdots\end{array}$ & $\begin{array}{l}\cdots \\
\cdots \\
\cdots \\
\cdots \\
\cdots \\
\cdots \\
\cdots\end{array}$ & $\begin{array}{l}6500 \pm 1200 \\
<5000 \\
6800 \pm 1000 \\
<5000 \\
<4000 \\
<4000 \\
6700 \pm 1700 \\
6000 \pm 1300\end{array}$ & $\begin{array}{l}1380 \pm 60 \\
2590 \pm 110 \\
1920 \pm 80 \\
2310 \pm 100 \\
2610 \pm 110 \\
2910 \pm 130 \\
2140 \pm 100 \\
2260 \pm 100\end{array}$ \\
\hline 1.0 & $\begin{array}{l}0 \\
6\end{array}$ & $\begin{array}{l}\cdots \\
\cdots \\
\cdots \\
\cdots\end{array}$ & $\begin{array}{l}\cdots \\
\cdots \\
\cdots \\
\cdots\end{array}$ & $\begin{array}{l}9300 \pm 1000 \\
5600 \pm 1300 \\
9100 \pm 1300 \\
9800 \pm 1400\end{array}$ & $\begin{aligned} 890 & \pm 40 \\
1270 & \pm 60 \\
970 & \pm 40 \\
1100 & \pm 50\end{aligned}$ \\
\hline 2.0 & 15 & $\begin{array}{l}\cdots \\
\cdots \\
\cdots \\
\cdots \\
\cdots \\
\cdots\end{array}$ & $\begin{array}{l}\cdots \\
\cdots \\
\cdots \\
\cdots \\
\cdots\end{array}$ & $\begin{array}{c}<3000 \\
<5000 \\
6100 \pm 1100 \\
6000 \pm 1600 \\
<4000 \\
4400 \pm 1500\end{array}$ & $\begin{array}{l}1840 \pm 80 \\
1580 \pm 70 \\
1630 \pm 70 \\
1870 \pm 80 \\
2250 \pm 100 \\
1900 \pm 90\end{array}$ \\
\hline 5.0 & 15 & $\begin{array}{l}\cdots \\
\cdots \\
\cdots \\
\cdots \\
\cdots\end{array}$ & $\begin{array}{l}\cdots \\
\cdots \\
\cdots \\
\cdots \\
\cdots\end{array}$ & $\begin{array}{c}<4000 \\
3900 \pm 900 \\
<4000 \\
<4000 \\
5100 \pm 1300 \\
<4000\end{array}$ & $\begin{array}{r}640 \pm 30 \\
1300 \pm 60 \\
1240 \pm 60 \\
1220 \pm 50 \\
1560 \pm 70 \\
1230 \pm 60\end{array}$ \\
\hline
\end{tabular}

$\mathrm{Na}(\%) \quad \mathrm{Nd}(u g / g) \quad \mathrm{pH}$ (units) Rb (ug/g) 


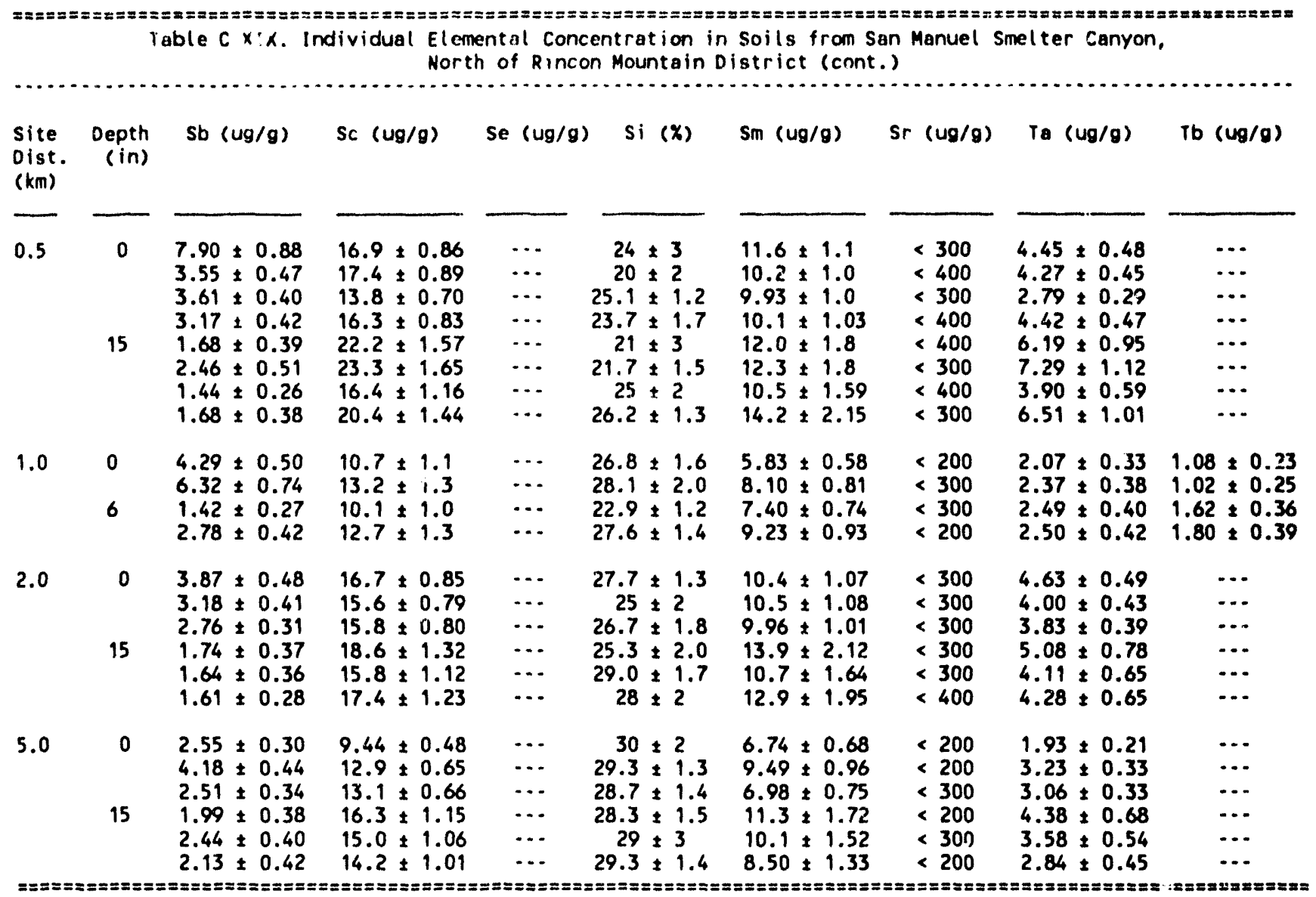

\begin{tabular}{|c|c|c|c|c|c|c|c|c|c|}
\hline $\begin{array}{l}\text { Site } \\
\text { Dist. } \\
\text { (km) }\end{array}$ & $\begin{array}{r}\text { Depth } \\
\text { (in) }\end{array}$ & Th (ug/g) & Ti $(x)$ & $U(u g / g)$ & $V($ (ug/g) & $W(u g / g)$ & $\mathrm{Yb}_{\mathrm{b}}(\mathrm{ug} / \mathrm{g})$ & $\operatorname{Zn}(u g / g)$ & $\operatorname{Zr}(\omega g / g)$ \\
\hline 0.5 & 15 & $\begin{array}{l}24.6 \pm 2.0 \\
17.7 \pm 1.4 \\
22.4 \pm 1.8 \\
28.2 \pm 2.2 \\
54.4 \pm 4.4 \\
21.8 \pm 1.7 \\
17.1 \pm 1.3 \\
30.5 \pm 2.4\end{array}$ & $\begin{array}{l}1.10 \pm 0.14 \\
1.96 \pm 0.11 \\
1.39 \pm 0.18 \\
1.84 \pm 0.10 \\
1.80 \pm 0.1 \\
2.24 \pm 0.12 \\
1.40 \pm 0.08 \\
1.85 \pm 0.10\end{array}$ & $\begin{array}{l}4.25 \pm 0.35 \\
3.95 \pm 0.3 \\
3.84 \pm 0.3 \\
3.69 \pm 0.3 \\
5.92 \pm 0.4 \\
5.71 \pm 0.4 \\
4.75 \pm 0.4 \\
5.84 \pm 0.4\end{array}$ & $\begin{array}{l}238 \pm 10 \\
331 \pm 14 \\
241 \pm 10 \\
308 \pm 13 \\
357 \pm 15 \\
419 \pm 18 \\
279 \pm 12 \\
364 \pm 15\end{array}$ & $\begin{array}{l}<150 \\
<200 \\
<160 \\
<200 \\
<180 \\
<200 \\
<170 \\
<190\end{array}$ & $\begin{array}{l}8.36 \pm 0.86 \\
7.73 \pm 0.79 \\
5.34 \pm 0.54 \\
7.52 \pm 0.77 \\
7.81 \pm 0.83 \\
9.20 \pm 0.95 \\
7.53 \pm 0.76 \\
9.74 \pm 1.01\end{array}$ & $\begin{array}{l}428 \pm 64 \\
234 \pm 35 \\
177 \pm 26 \\
154 \pm 23 \\
166 \pm 33 \\
190 \pm 38 \\
111 \pm 22 \\
154 \pm 31\end{array}$ & $\begin{array}{l}\cdots \\
\cdots \\
\cdots \\
\cdots \\
\cdots \\
\cdots \\
\cdots\end{array}$ \\
\hline 1.0 & $\begin{array}{l}0 \\
6\end{array}$ & $\begin{array}{l}12.1 \pm 0.6 \\
23.0 \pm 1.2 \\
14.0 \pm 0.7 \\
16.4 \pm 0.9\end{array}$ & $\begin{array}{l}0.82 \pm 0.11 \\
1.15 \pm 0.07 \\
0.94 \pm 0.05 \\
1.09 \pm 0.06\end{array}$ & $\begin{array}{r}2.83 \pm 0.2 \\
3.76 \pm 0.3 \\
3.54 \pm 0.3 \\
3.5 \pm 0.3\end{array}$ & $\begin{array}{l}115 \pm 5 \\
169 \pm 8 \\
142 \pm 6 \\
182 \pm 8\end{array}$ & $\begin{array}{l}<110 \\
<160 \\
<120 \\
<140\end{array}$ & $\begin{array}{l}4.60 \pm 0.47 \\
4.93 \pm 0.52 \\
4.22 \pm 0.47 \\
5.33 \pm 0.58\end{array}$ & $\begin{aligned} 205 & \pm 21 \\
243 & \pm 25 \\
94 & \pm 14 \\
149 & \pm 23\end{aligned}$ & $\begin{array}{l}\cdots \\
\cdots \\
\cdots \\
\cdots\end{array}$ \\
\hline 2.0 & 15 & $\begin{array}{l}23.2 \pm 1.8 \\
21.7 \pm 1.7 \\
44.7 \pm 3.6 \\
26.8 \pm 2.1 \\
26.1 \pm 2.1 \\
27.0 \pm 2.1\end{array}$ & $\begin{array}{l}1.45 \pm 0.19 \\
1.15 \pm 0.07 \\
1.26 \pm 0.16 \\
1.35 \pm 0.08 \\
1.26 \pm 0.07 \\
1.50 \pm 0.09\end{array}$ & $\begin{array}{l}4.36 \pm 0.35 \\
4.31 \pm 0.35 \\
4.28 \pm 0.35 \\
3.76 \pm 0.3 \\
4.35 \pm 0.3 \\
4.55 \pm 0.4\end{array}$ & $\begin{array}{l}231 \pm 10 \\
221 \pm 10 \\
245 \pm 10 \\
230 \pm 10 \\
187 \pm 8 \\
211 \pm 9\end{array}$ & $\begin{array}{l}<150 \\
<180 \\
<150 \\
<150 \\
<200 \\
<160\end{array}$ & $\begin{array}{l}7.81 \pm 0.80 \\
7.17 \pm 0.74 \\
7.30 \pm 0.74 \\
9.52 \pm 0.99 \\
6.56 \pm 0.70 \\
7.08 \pm 0.72\end{array}$ & $\begin{array}{l}172 \pm 26 \\
204 \pm 31 \\
169 \pm 25 \\
120 \pm 24 \\
104 \pm 21 \\
112 \pm 22\end{array}$ & $\begin{array}{l}\cdots \\
\cdots \\
\cdots \\
\cdots \\
\cdots\end{array}$ \\
\hline 5.0 & 15 & $\begin{array}{l}10.3 \pm 0.8 \\
32.1 \pm 2.5 \\
13.6 \pm 1.1 \\
21.0 \pm 1.7 \\
20.2 \pm 1.6 \\
13.5 \pm 1.1\end{array}$ & $\begin{array}{l}0.93 \pm 0.06 \\
1.48 \pm 0.19 \\
1.29 \pm 0.07 \\
1.52 \pm 0.08 \\
1.61 \pm 0.09 \\
1.28 \pm 0.07\end{array}$ & $\begin{array}{l}4.74 \pm 0.4 \\
3.26 \pm 0.3 \\
2.72 \pm 0.2 \\
3.47 \pm 0.3 \\
3.43 \pm 0.3 \\
4.35 \pm 0.35\end{array}$ & $\begin{array}{r}92 \pm 5 \\
216 \pm 9 \\
168 \pm 8 \\
217 \pm 9 \\
204 \pm 9 \\
180 \pm 8\end{array}$ & $\begin{array}{l}<120 \\
<130 \\
<160 \\
<150 \\
<150 \\
<150\end{array}$ & $\begin{array}{l}4.03 \pm 0.41 \\
6.23 \pm 0.63 \\
4.09 \pm 0.44 \\
5.32 \pm 0.56 \\
5.12 \pm 0.53 \\
5.515 \pm 0.59\end{array}$ & $\begin{array}{r}74 \pm 11 \\
118 \pm 17 \\
105 \pm 16 \\
103 \pm 20 \\
98 \pm 19 \\
140 \pm 28\end{array}$ & $\begin{array}{l}\cdots \\
\cdots \\
\cdots \\
\cdots \\
\cdots \\
\cdots\end{array}$ \\
\hline
\end{tabular}


Iable C-xx. Individual Elemental Concentration in Soils from Iucson Mountain District, Saguaro National Monument

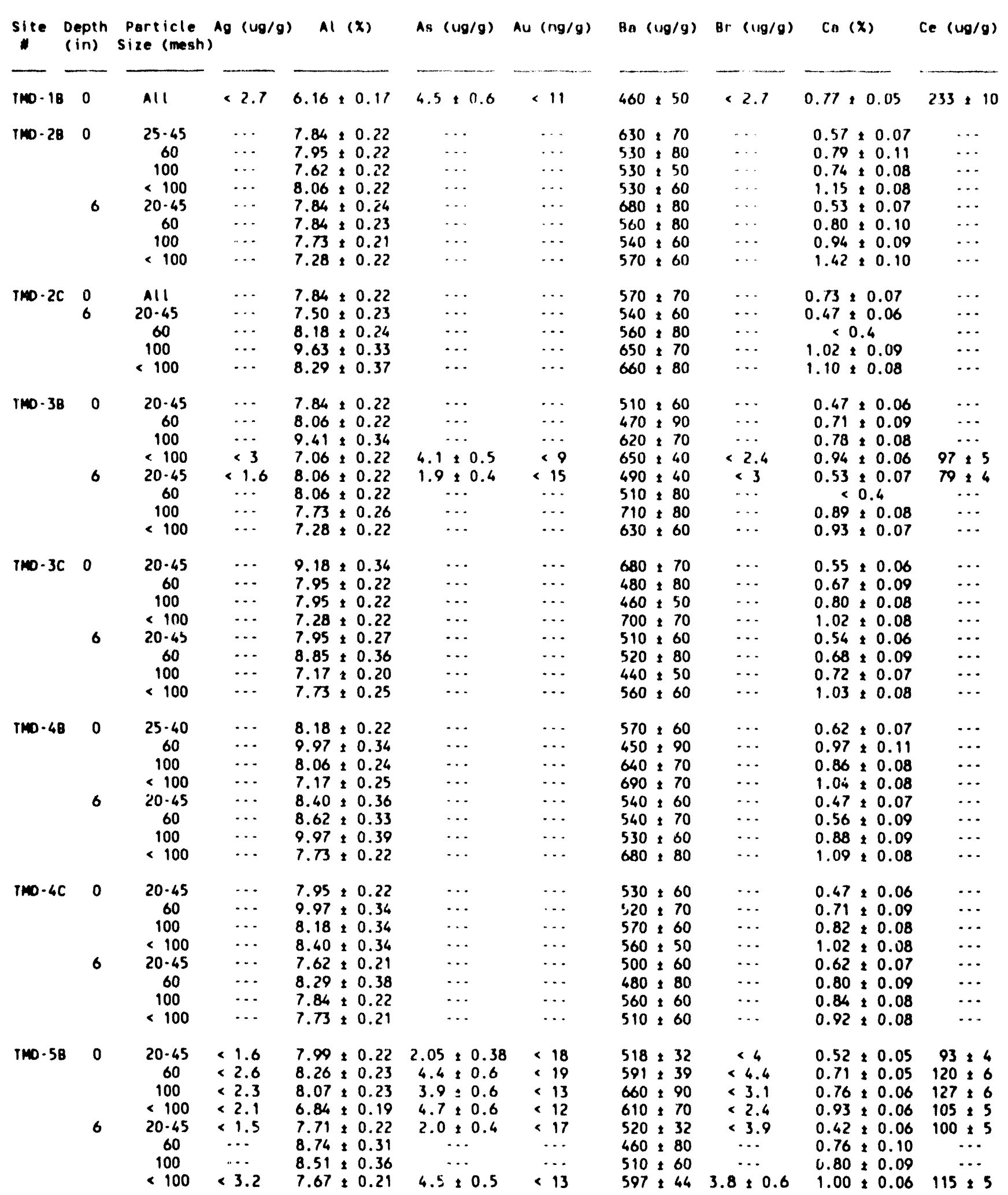




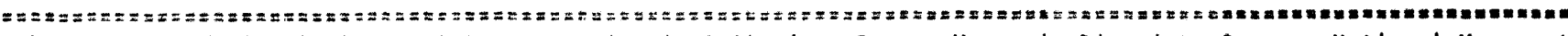
Table C.Xx. Individual Elemental Concentration in Soils from Tucson Mountain District, Saguaro National Monument (cont.)

\begin{tabular}{|c|c|c|c|c|c|c|c|c|c|c|c|c|c|}
\hline $\begin{array}{c}\text { site } \\
0\end{array}$ & $\begin{array}{l}\text { Depth } \\
\text { (in) }\end{array}$ & $\begin{array}{l}\text { Particle } \\
\text { size (mesh) }\end{array}$ & $\operatorname{Ag}(u g / g)$ & Al & $(x)$ & As ( & $(u g / g)$ & Au $(n g / \theta)$ & Ba $(u g / g)$ & $\operatorname{Br}(u g / g)$ & Ce & $(x)$ & Ce $(\omega 0 / 0)$ \\
\hline$T M O \cdot 5 C$ & 0 & $\begin{array}{c}20 \cdot 45 \\
60 \\
100 \\
<100 \\
20 \cdot 45 \\
60 \\
100 \\
<100\end{array}$ & $\begin{array}{l}\cdots \\
\cdots \\
\cdots \\
\cdots \\
\cdots \\
\cdots \\
\cdots\end{array}$ & $\begin{array}{l}7.95 \\
8.29 \\
7.95 \\
7.95 \\
7.62 \\
7.62 \\
7.17 \\
8.18\end{array}$ & $\begin{array}{l}+0.21 \\
+0.33 \\
+0.25 \\
+0.22 \\
+0.24 \\
+0.26 \\
+0.22 \\
+0.22\end{array}$ & & $\begin{array}{l}\ldots \\
\ldots \\
\ldots \\
\ldots \\
\ldots \\
\cdots \\
\cdots\end{array}$ & $\begin{array}{l}\ldots \\
\ldots \\
\ldots \\
\ldots \\
\ldots \\
\cdots \\
\ldots\end{array}$ & $\begin{array}{c}480: 60 \\
510: 80 \\
570: 60 \\
580: 70 \\
\cdots \\
490: 70 \\
470: 50 \\
540: 70\end{array}$ & $\begin{array}{l}\ldots \\
\cdots \\
\ldots \\
\cdots \\
\cdots \\
\cdots \\
\cdots\end{array}$ & $\begin{array}{l}0.58 \\
0.50 \\
0.68 \\
1.04 \\
0.72 \\
0.69 \\
0.94\end{array}$ & $\begin{array}{l}\quad 0.07 \\
+0.08 \\
+0.07 \\
0.08 \\
+0.09 \\
+0.07 \\
+0.08\end{array}$ & $\begin{array}{l}\ldots \\
\cdots \\
\ldots \\
\ldots \\
\ldots \\
\ldots \\
\ldots\end{array}$ \\
\hline TMD - 6 & 0 & $\begin{array}{c}20-45 \\
60 \\
100 \\
<100 \\
20-45 \\
60 \\
100 \\
<100\end{array}$ & $\begin{array}{l}<1.3 \\
<1.6 \\
<2 \\
<2 \\
<1.5 \\
<2.9 \\
<2.5\end{array}$ & $\begin{array}{l}6.82 \\
7.14 \\
6.93 \\
6.67 \\
6.80 \\
7.39 \\
7.28 \\
7.13\end{array}$ & $\begin{array}{l}\text { t } 0.19 \\
+0.20 \\
+0.17 \\
t 0.19 \\
+0.19 \\
+0.22 \\
+0.20 \\
+0.20\end{array}$ & $\begin{array}{r}1.79 \\
2.28 \\
4.2 \\
4.3 \\
3.18 \\
3.76 \\
5.7\end{array}$ & $\begin{array}{l}t 0.35 \\
t 0.38 \\
t 0.5 \\
t 0.5 \\
t 0.43 \\
\therefore \\
10.49 \\
10.6\end{array}$ & $\begin{array}{l}<11 \\
<14 \\
<15 \\
<12 \\
<14 \\
\cdots \\
<15 \\
<9\end{array}$ & $\begin{array}{l}529: 33 \\
504: 31 \\
580: 60 \\
700: 70 \\
525: 30 \\
410: 70 \\
628: 46 \\
658: 43\end{array}$ & $\begin{array}{l}2.8 \\
<2.8 \\
<3.6 \\
<2.3 \\
<3.2 \\
\cdots \\
\times 3.6 \\
<2.2\end{array}$ & $\begin{array}{r}0.53 \\
0.67 \\
0.69 \\
1.00 \\
0.47 \\
< \\
0.78 \\
0.90\end{array}$ & $\begin{array}{l}+0.06 \\
+0.05 \\
+0.05 \\
+0.06 \\
+0.04 \\
0.4 \\
+0.05 \\
+0.06\end{array}$ & $\begin{array}{r}48: 2 \\
82: 4 \\
117: 5 \\
85: 4 \\
61: 3 \\
\cdots \\
115: 5 \\
101: 5\end{array}$ \\
\hline TMD-7 & 0 & $\begin{array}{c}20 \cdot 45 \\
60 \\
100 \\
<100 \\
20 \cdot 45 \\
60 \\
100 \\
<100\end{array}$ & $\begin{array}{l}<1.8 \\
<2.5 \\
<3.4 \\
<2.5 \\
<1.7 \\
<2.5 \\
<3.5 \\
<2.7\end{array}$ & $\begin{array}{l}6.47 \\
6.51 \\
6.64 \\
6.58 \\
6.82 \\
6.81 \\
6.80 \\
7.26\end{array}$ & $\begin{array}{l}+0.18 \\
+0.18 \\
+0.19 \\
+0.18 \\
+0.19 \\
+0.19 \\
+0.19 \\
10.20\end{array}$ & $\begin{array}{r}2.73 \\
4.7 \\
4.9 \\
5.3 \\
2.84 \\
5.4 \\
5.9 \\
6.5\end{array}$ & $\begin{array}{l}10.40 \\
10.6 \\
10.6 \\
10.6 \\
10.43 \\
10.6 \\
10.7 \\
10.7\end{array}$ & $\begin{array}{l}<14 \\
<15 \\
<14 \\
<9 \\
<13 \\
<14 \\
<14 \\
<8\end{array}$ & $\begin{array}{l}551: 34 \\
514: 43 \\
630: 90 \\
600: 80 \\
554: 35 \\
480: 38 \\
600: 120 \\
566: 40\end{array}$ & $\begin{array}{c}<2.7 \\
<3.3 \\
<3.5 \\
1.1 \neq 0.3 \\
1.6: 0.7 \\
2.0: 0.5 \\
1.8: 0.3 \\
2.8 \neq 0.4\end{array}$ & $\begin{array}{l}0.57 \\
0.79 \\
0.82 \\
0.99 \\
0.49 \\
0.79 \\
0.71 \\
0.96\end{array}$ & $\begin{array}{l}+0.05 \\
+0.06 \\
+0.06 \\
+0.06 \\
+0.04 \\
+0.05 \\
+0.05 \\
+0.06\end{array}$ & $\begin{array}{r}104: 5 \\
134: 6 \\
126: 6 \\
102: 5 \\
96: 4 \\
126: 6 \\
130: 6 \\
99: 5\end{array}$ \\
\hline
\end{tabular}

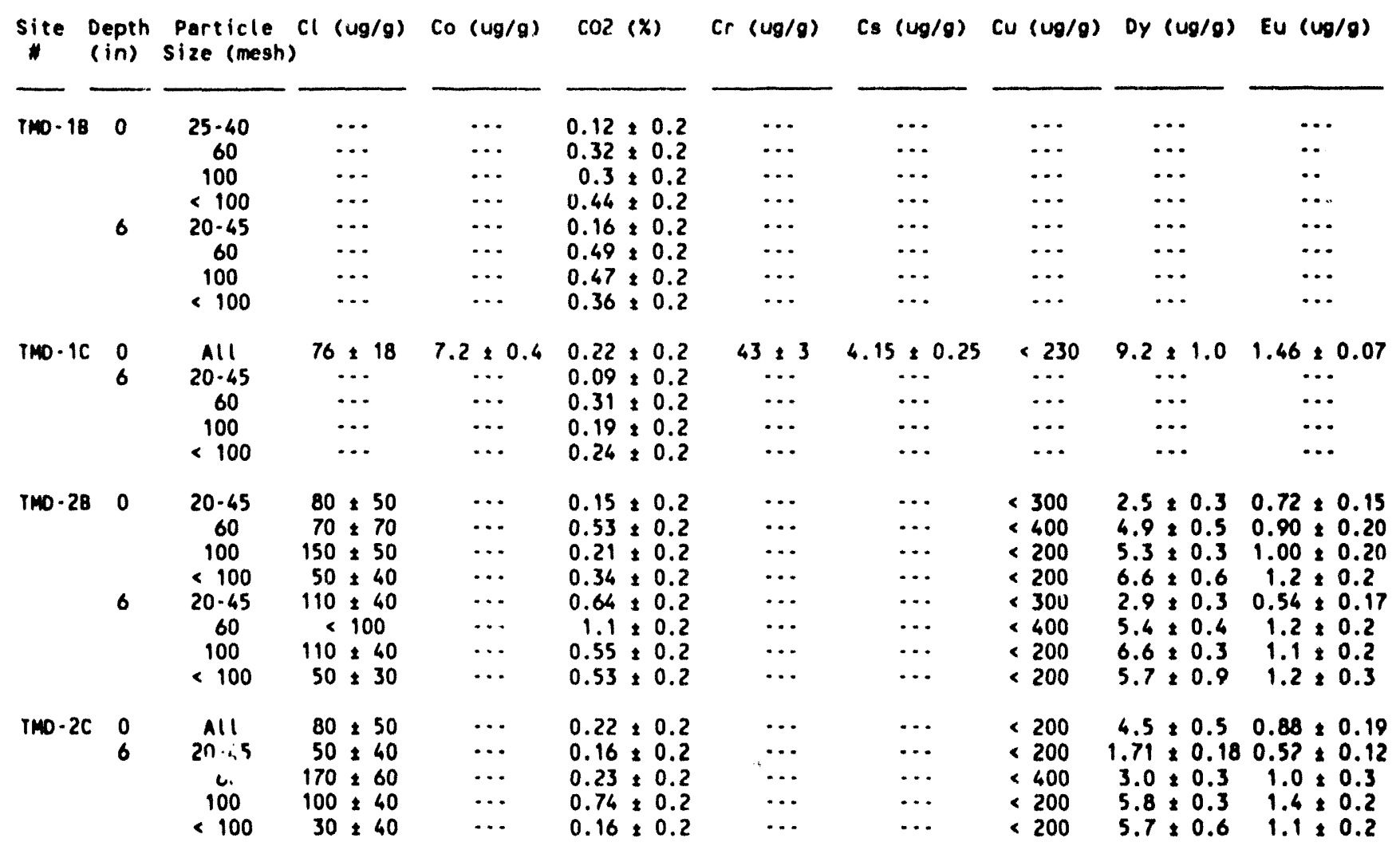




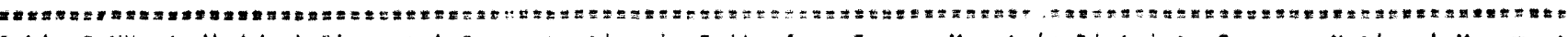
Iable C-XX. Individual Elemental Concentration in Solls from Tucson Mountain District, Saguaro Mational Monument (cont.)

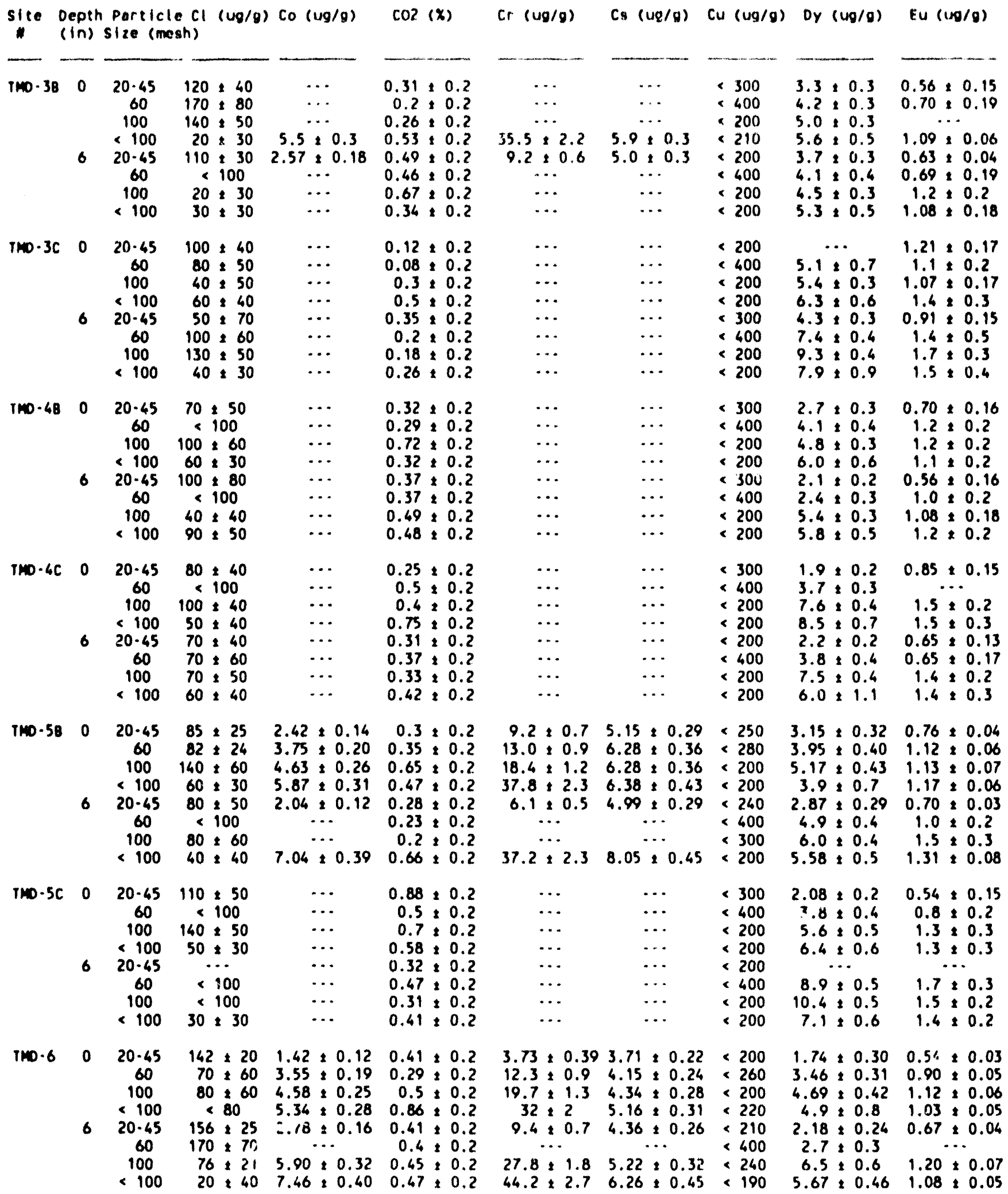




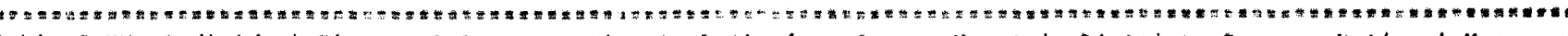
Iable C.XX. Individual Elemental Concentration in Solls from tucson Mountain District, Saguaro Natlonal Monument (cont.)

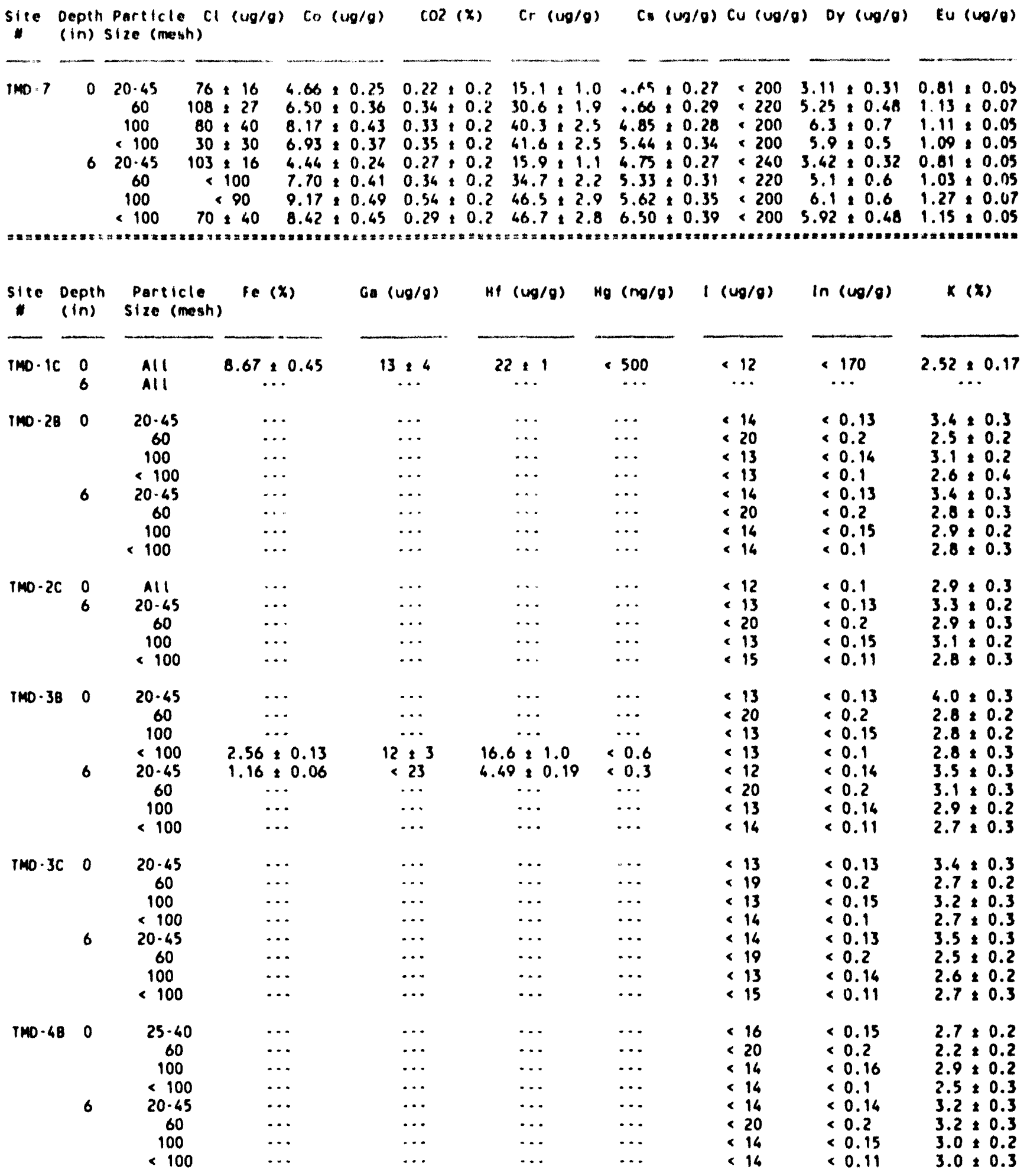




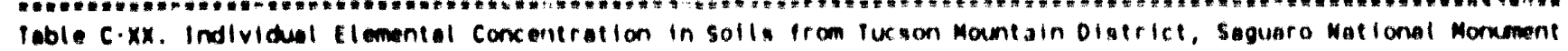
(cont.)

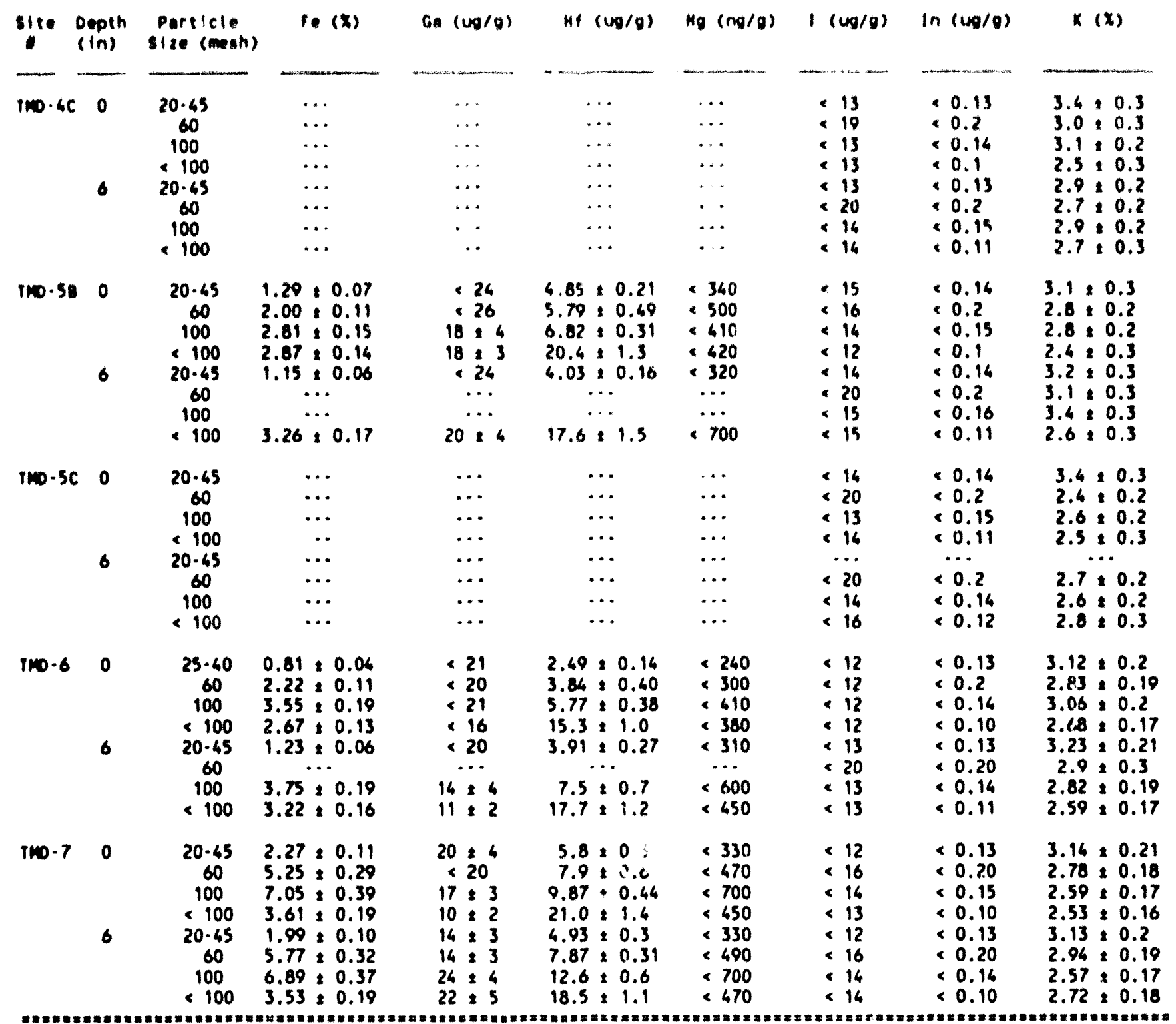

\begin{tabular}{|c|c|c|c|c|c|c|c|c|c|}
\hline site & $\begin{array}{l}\text { Depth } \\
(\text { in) }\end{array}$ & $\begin{array}{l}\text { Particle } \\
\text { size(mesh) }\end{array}$ & Lo (ug/g) & $\operatorname{lu}(u g / 3)$ & $M g(u g / g)$ & $\operatorname{Mn}(u g / g)$ & $\mathrm{Na}(\mathrm{X})$ & Nd $(u g / g)$ & $R b(u g / g$ \\
\hline $\begin{array}{l}\text { THO- } 18 \\
T M D-1 C \\
T H D-28\end{array}$ & $\begin{array}{ll}8 & 0 \\
& 6 \\
C & 0 \\
& 6 \\
& 6\end{array}$ & $\begin{array}{c}A 11 \\
A 11 \\
A 11 \\
A 11 \\
20-45 \\
60 \\
100 \\
<100 \\
20.45 \\
60 \\
100 \\
<100\end{array}$ & $\begin{array}{c}\cdots \\
\cdots \\
136 \\
\ldots \\
\cdots \\
\cdots \\
\cdots \\
\cdots \\
\cdots \\
\cdots \\
\cdots\end{array}$ & $\begin{array}{c}\ldots \\
\ldots \\
1050 \\
\ldots \\
\ldots \\
\ldots \\
\ldots \\
\ldots \\
\ldots \\
\ldots \\
\ldots \\
\ldots \\
\ldots\end{array}$ & $\begin{array}{c}\cdots \\
\cdots \\
1970 \neq 390 \\
\cdots \\
<3000 \\
<5000 \\
4100 \neq 1200 \\
4500 \pm 800 \\
33000 \pm 12000 \\
<5000 \\
<3000 \\
4300 \pm 700\end{array}$ & $\begin{array}{c}\cdots \\
389: 16 \\
\cdots \\
320 \div 15 \\
392 \div 19 \\
425 \div 20 \\
470 \div 20 \\
314 \div 16 \\
380 \div 30 \\
431: 20 \\
470 \div 20\end{array}$ & $\begin{array}{c}\cdots \\
\cdots \\
2.32 \div 0.10 \\
\cdots \\
2.87 \div 0.09 \\
2.88 \div 0.10 \\
2.35 \div 0.08 \\
1.79 \div 0.06 \\
2.77 \div 0.09 \\
2.86 \div 0.10 \\
2.35 \div 0.08 \\
1.68 \div 0.06\end{array}$ & $\begin{array}{c}\ldots \\
78 \\
\ldots \\
\ldots \\
\cdots \\
\cdots \\
\cdots \\
\cdots \\
\cdots \\
\cdots\end{array}$ & $\begin{array}{c}\ldots \\
\cdots \\
134 \\
\ldots \\
\ldots \\
\cdots \\
\cdots \\
\ldots \\
\ldots \\
\ldots \\
\ldots\end{array}$ \\
\hline
\end{tabular}




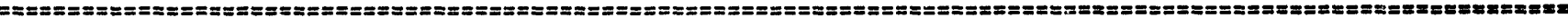
Table C-XX. Individual Elemental Concentration in Soils from Tucson Mountain District, Saguaro National Monument (cont.)

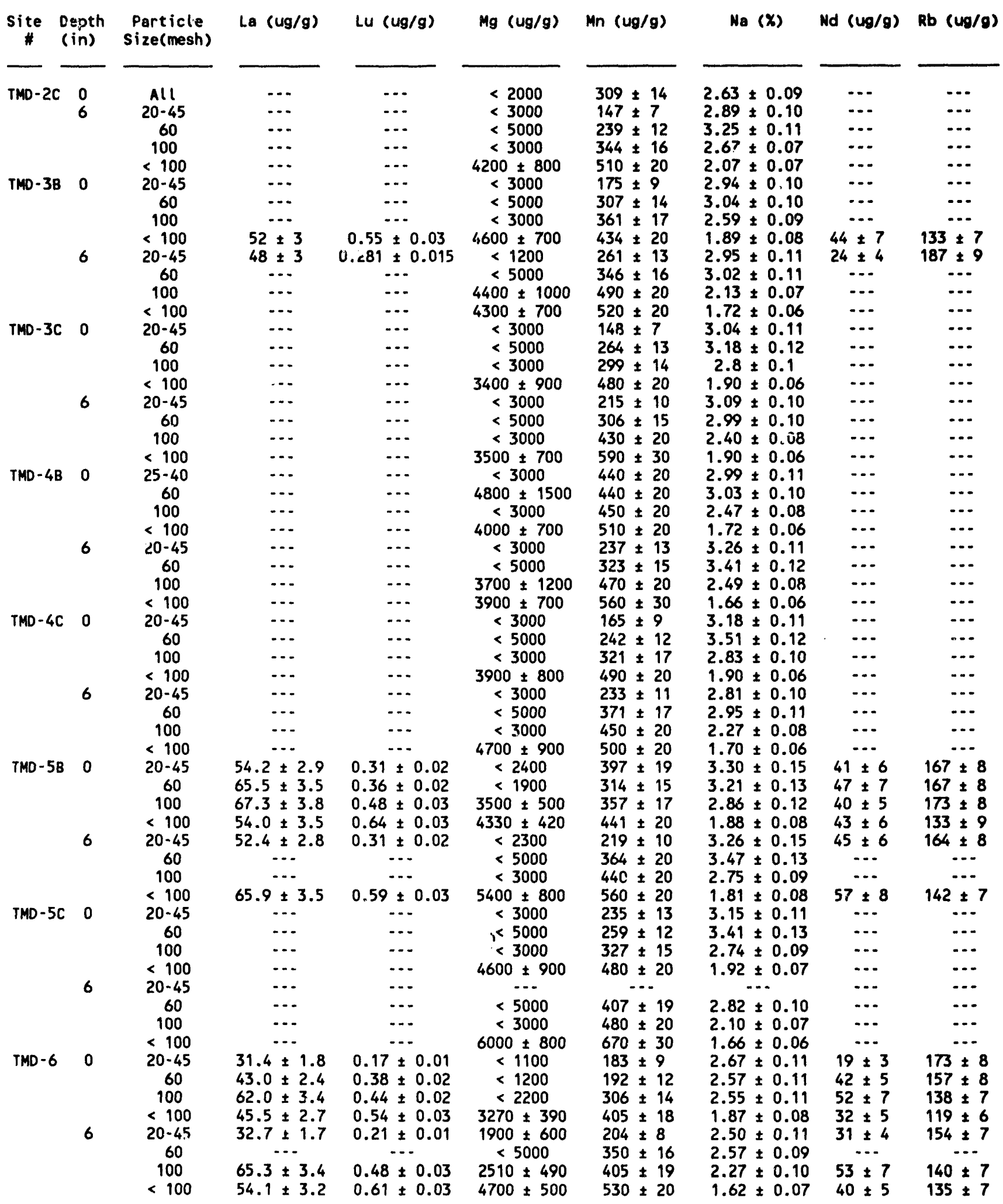




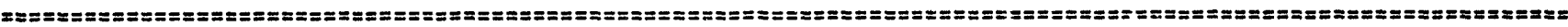
Table C-XX. Individual Elemental Concentration in Soils from Tucson Mountain District, Saguaro National Monument (cont.)

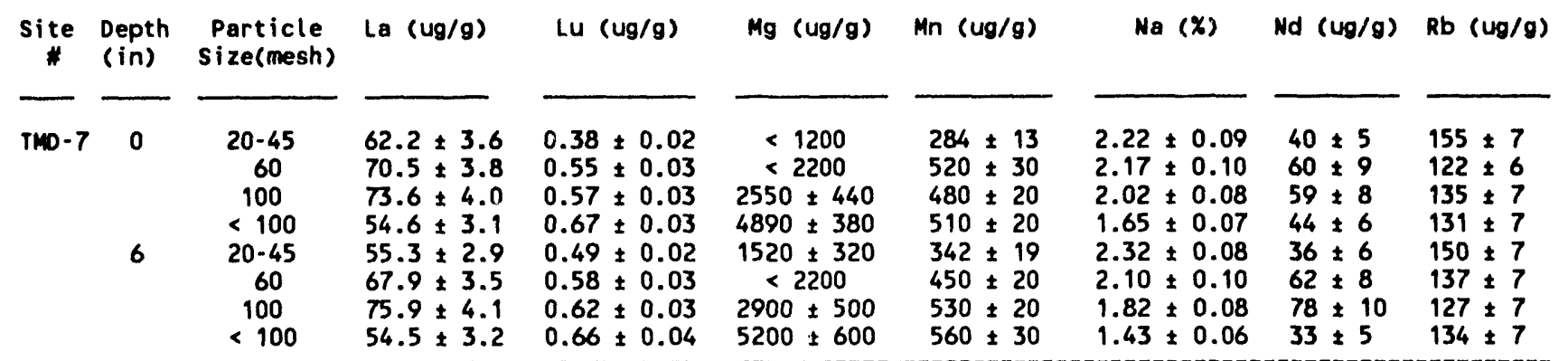

\begin{tabular}{|c|c|c|c|c|c|c|c|c|c|}
\hline $\begin{array}{cc}\text { Site } & 0 \\
\# & (\end{array}$ & $\begin{array}{l}\text { Depth } \\
\text { (in) }\end{array}$ & $\begin{array}{c}\text { Particle } \\
\text { size(mesh) }\end{array}$ & $\mathrm{Sb}(u g / g)$ & Sc $(u g / g)$ & Se $(u g / g)$ & Sm (ug/g) & Sr $(u g / g)$ & To (ug/g) & Tb $(1 \mathrm{gg} / \mathrm{g})$ \\
\hline \multirow[t]{2}{*}{ TMD - 1B } & 0 & All & $\cdots$ & $\cdots$ & $\cdots$ & $\cdots$ & $\cdots$ & $\cdots$ & $\cdots$ \\
\hline & 6 & All & $\cdots$ & $\cdots$ & $\ldots$ & $\cdots$ & $\cdots$ & $\cdots$ & $\ldots$ \\
\hline \multirow[t]{2}{*}{ TMD - IC } & 0 & All & $1.20 \pm 0.11$ & $6.42 \pm 0.34$ & $<2$ & $13.1 \pm 0.6$ & $<200$ & $4.32 \pm 0.28$ & $1.32 \pm 0.12$ \\
\hline & 6 & All & $\ldots$ & $\ldots$ & $\cdots$ & $\cdots$ & $\cdots$ & $\ldots$ & $\cdots$ \\
\hline \multirow[t]{8}{*}{$T M D-2 B$} & 0 & $20-45$ & $\cdots$ & $\cdots$ & $\cdots$ & $\cdots$ & $<200$ & $\cdots$ & $\cdots$ \\
\hline & & 60 & $\cdots$ & $\cdots$ & $\cdots$ & $\cdots$ & $<300$ & $\cdots$ & $\cdots$ \\
\hline & & 100 & $\cdots$ & $\cdots$ & $\cdots$ & $\cdots$ & $<190$ & $\cdots$ & $\cdots$ \\
\hline & & $<100$ & $\cdots$ & $\cdots$ & $\cdots$ & $\cdots$ & $<200$ & $\cdots$ & $\ldots$ \\
\hline & 6 & $20-45$ & $\cdots$ & $\cdots$ & $\cdots$ & $\cdots$ & $<200$ & $\cdots$ & $\cdots$ \\
\hline & & 60 & $\cdots$ & $\cdots$ & $\cdots$ & $\cdots$ & $330 \pm 150$ & $\cdots$ & $\cdots$ \\
\hline & & 100 & $\cdots$ & $\cdots$ & $\cdots$ & $\cdots$ & $<200$ & $\cdots$ & $\cdots$ \\
\hline & & $<100$ & $\cdots$ & $\cdots$ & $\cdots$ & $\cdots$ & $<200$ & $\cdots$ & $\cdots$ \\
\hline \multirow[t]{5}{*}{$T M D-2 C$} & 0 & All & $\cdots$ & $\cdots$ & $\cdots$ & $\cdots$ & $<190$ & $\cdots$ & $\cdots$ \\
\hline & 6 & $20-45$ & $\cdots$ & $\cdots$ & $\cdots$ & $\cdots$ & $260 \pm 80$ & $\cdots$ & $\cdots$ \\
\hline & & 60 & $\cdots$ & $\cdots$ & $\cdots$ & $\cdots$ & $<300$ & $\cdots$ & $\cdots$ \\
\hline & & 100 & $\cdots$ & $\cdots$ & $\cdots$ & $\cdots$ & $<190$ & $\cdots$ & $\cdots$ \\
\hline & & $<100$ & $\cdots$ & $\cdots$ & $\cdots$ & $\cdots$ & $<200$ & $\cdots$ & $\cdots$ \\
\hline \multirow[t]{7}{*}{ TMD - 3B } & 0 & $20-45$ & $\cdots$ & $\cdots$ & $\cdots$ & $\cdots$ & $<200$ & $\cdots$ & $\cdots$ \\
\hline & & 60 & $\cdots$ & $\cdots$ & $\cdots$ & $\cdots$ & $<300$ & $\cdots$ & $\cdots$ \\
\hline & & 100 & $\cdots$ & $\ldots$ & $\cdots$ & $\ldots$ & $<190$ & $\cdots$ & $\cdots$ \\
\hline & & $<100$ & $1.48 \pm 0.12$ & $7.3 \pm 0.4$ & $<3$ & $6.5 \pm 0.3$ & $390 \pm 100$ & $1.64 \pm 0.10$ & $0.78 \pm 0.08$ \\
\hline & 6 & $\begin{array}{c}20-45 \\
60\end{array}$ & $\begin{array}{c}0.91 \pm 0.11 \\
\ldots\end{array}$ & $\begin{array}{c}3.25 \pm 0.18 \\
\ldots\end{array}$ & $<1.7$ & $4.02 \pm 0.19$ & $\begin{array}{l}<200 \\
<300\end{array}$ & $1.58 \pm 0.10$ & $0.39 \pm 0.04$ \\
\hline & & $\begin{array}{r}60 \\
100\end{array}$ & $\cdots$ & $\ldots$ & $\cdots$ & $\cdots$ & $<190$ & $\ldots$ & $\ldots$ \\
\hline & & $<100$ & $\cdots$ & $\cdots$ & $\ldots$ & $\cdots$ & $<200$ & $\cdots$ & $\cdots$ \\
\hline \multirow[t]{8}{*}{$T M D-3 C$} & 0 & $20-45$ & $\cdots$ & $\cdots$ & $\cdots$ & $\cdots$ & $<200$ & $\cdots$ & $\cdots$ \\
\hline & & 60 & $\cdots$ & $\cdots$ & $\cdots$ & $\cdots$ & $<300$ & $\cdots$ & $\cdots$ \\
\hline & & 100 & $\cdots$ & $\cdots$ & $\cdots$ & $\cdots$ & $<190$ & $\cdots$ & $\cdots$ \\
\hline & & $<100$ & $\ldots$ & $\ldots$ & $\cdots$ & $\cdots$ & $<200$ & $\cdots$ & $\cdots$ \\
\hline & 6 & $20-45$ & $\cdots$ & $\cdots$ & $\cdots$ & $\cdots$ & $340 \pm 110$ & $\cdots$ & $\cdots$ \\
\hline & & 60 & $\cdots$ & $\cdots$ & $\cdots$ & $\cdots$ & $<300$ & $\cdots$ & $\cdots$ \\
\hline & & 100 & $\cdots$ & $\cdots$ & $\cdots$ & $\cdots$ & $<190$ & $\cdots$ & $\cdots$ \\
\hline & & $<100$ & $\cdots$ & $\cdots$ & $\cdots$ & $\cdots$ & $<200$ & $\cdots$ & $\cdots$ \\
\hline \multirow[t]{8}{*}{$T M D-4 B$} & 0 & $25-40$ & $\cdots$ & $\cdots$ & $\cdots$ & $\cdots$ & $<300$ & $\ldots$ & $\ldots$ \\
\hline & & 60 & $\cdots$ & $\cdots$ & $\cdots$ & $\cdots$ & $<300$ & $\cdots$ & $\cdots$ \\
\hline & & 100 & $\cdots$ & $\cdots$ & $\cdots$ & $\cdots$ & $<300$ & $\cdots$ & $\cdots$ \\
\hline & & $<100$ & $\cdots$ & $\cdots$ & $\cdots$ & $\cdots$ & $230 \pm 80$ & $\cdots$ & $\cdots$ \\
\hline & 6 & $20-45$ & $\cdots$ & $\cdots$ & $\cdots$ & $\cdots$ & $<200$ & $\cdots$ & $\cdots$ \\
\hline & & 60 & $\cdots$ & $\cdots$ & $\cdots$ & $\cdots$ & $<300$ & $\cdots$ & $\ldots$ \\
\hline & & 100 & $\cdots$ & $\ldots$ & $\cdots$ & $\cdots$ & $300 \pm 110$ & $\cdots$ & $\cdots$ \\
\hline & & $<100$ & $\cdots$ & $\cdots$ & $\cdots$ & $\cdots$ & $<200$ & $\cdots$ & $\cdots$ \\
\hline \multirow[t]{8}{*}{$T M D-4 C$} & 0 & $20-45$ & $\cdots$ & $\cdots$ & $\cdots$ & $\cdots$ & $<200$ & $\cdots$ & $\cdots$ \\
\hline & & 60 & $\cdots$ & $\cdots$ & $\cdots$ & $\cdots$ & $<190$ & $\cdots$ & $\cdots$ \\
\hline & & 100 & $\cdots$ & $\cdots$ & $\cdots$ & $\cdots$ & $<180$ & $\cdots$ & $\cdots$ \\
\hline & & $<100$ & $\cdots$ & $\cdots$ & $\cdots$ & $\cdots$ & $270 \pm 90$ & $\cdots$ & $\cdots$ \\
\hline & 6 & $20-45$ & $\cdots$ & $\cdots$ & $\cdots$ & $\cdots$ & $<200$ & $\cdots$ & $\cdots$ \\
\hline & & 60 & $\cdots$ & $\cdots$ & $\cdots$ & $\cdots$ & $<300$ & $\cdots$ & $\ldots$ \\
\hline & & 100 & $\cdots$ & $\cdots$ & $\cdots$ & $\cdots$ & $260 \pm 80$ & $\cdots$ & $\cdots$ \\
\hline & & $<100$ & $\cdots$ & $\cdots$ & $\cdots$ & $\cdots$ & $400 \pm 110$ & $\cdots$ & $\cdots$ \\
\hline
\end{tabular}




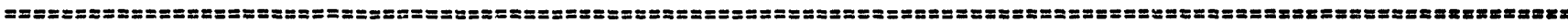
Table C-XX. Individual Elemental Concentration in Soils from Tucson Mountain District, Saguaro Mational Monument (cont.)

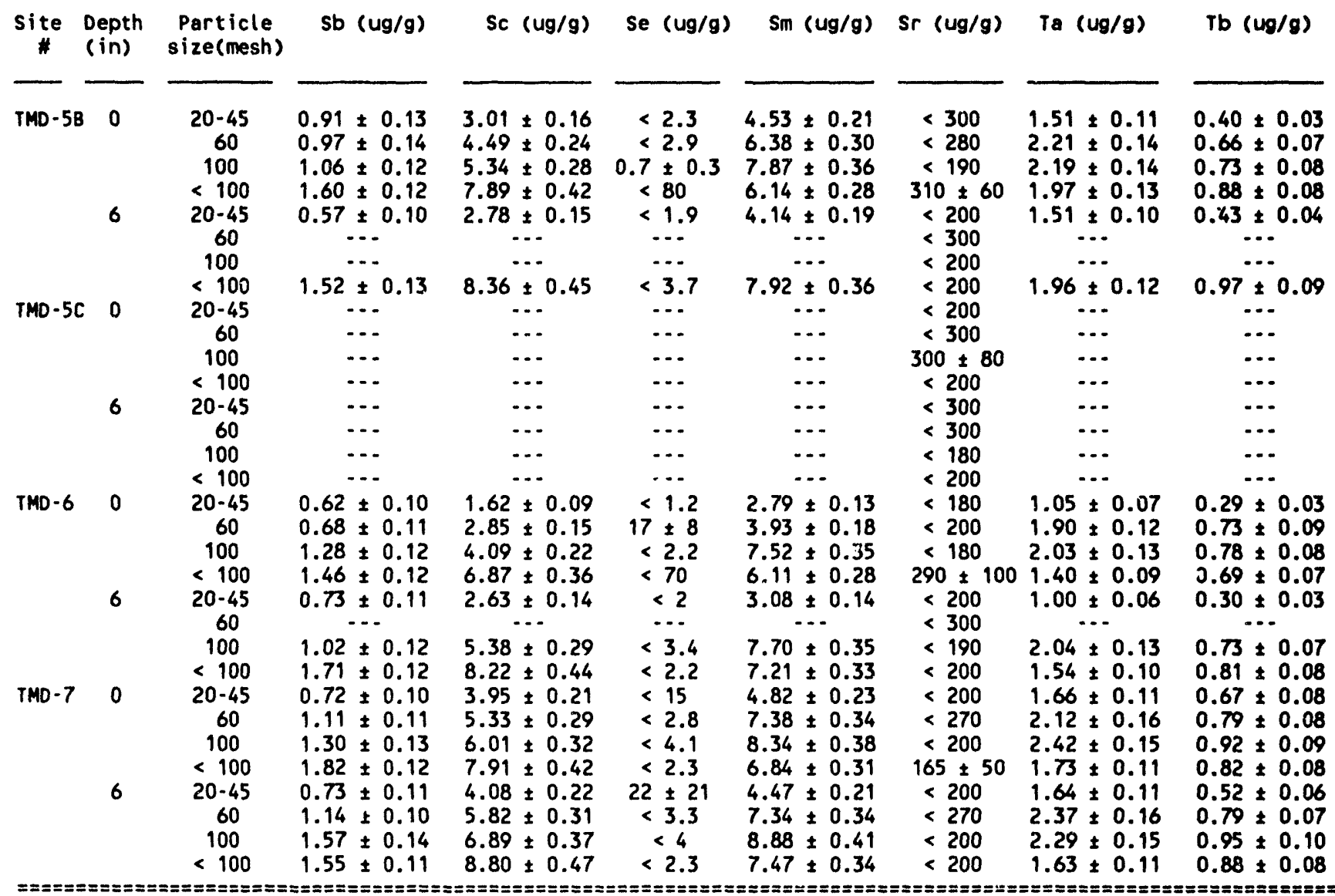

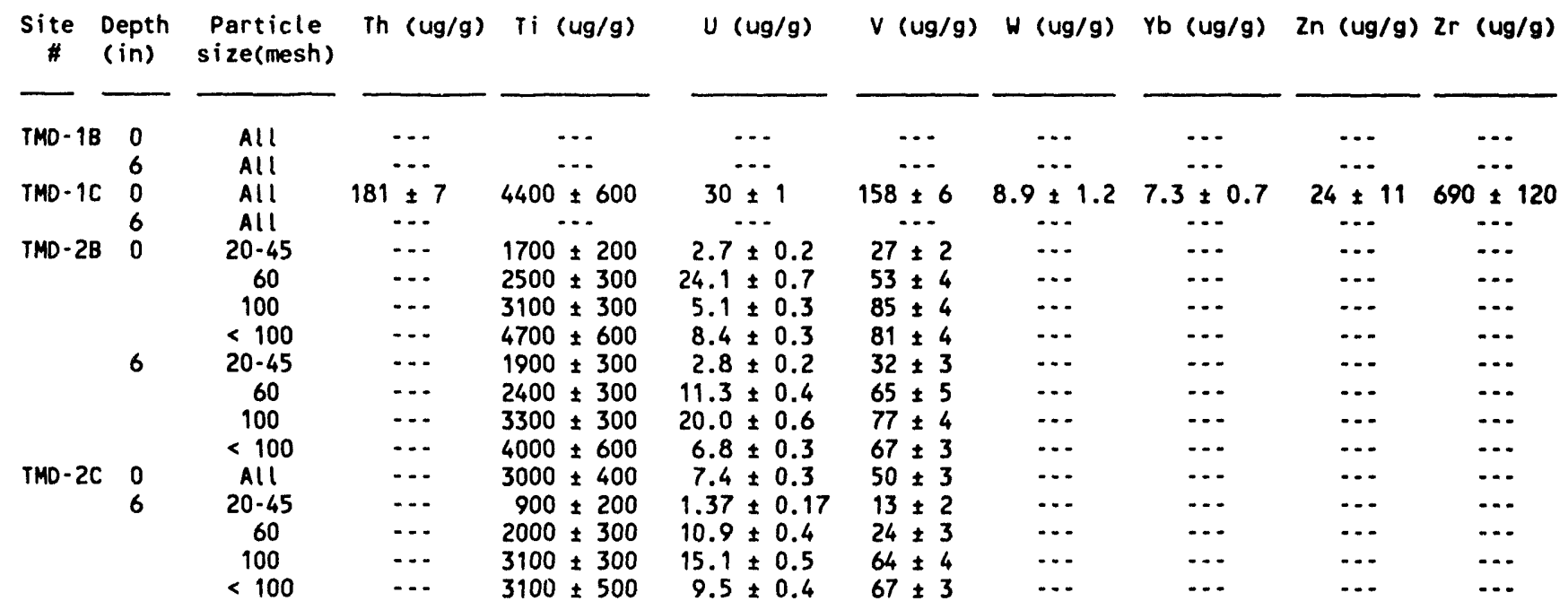




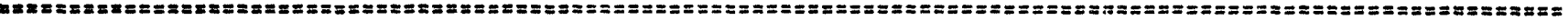
Table C.XX. Individual Elemental Concentration in Soils fron Tucson Mountain District, Saguaro National Monument (cont.)

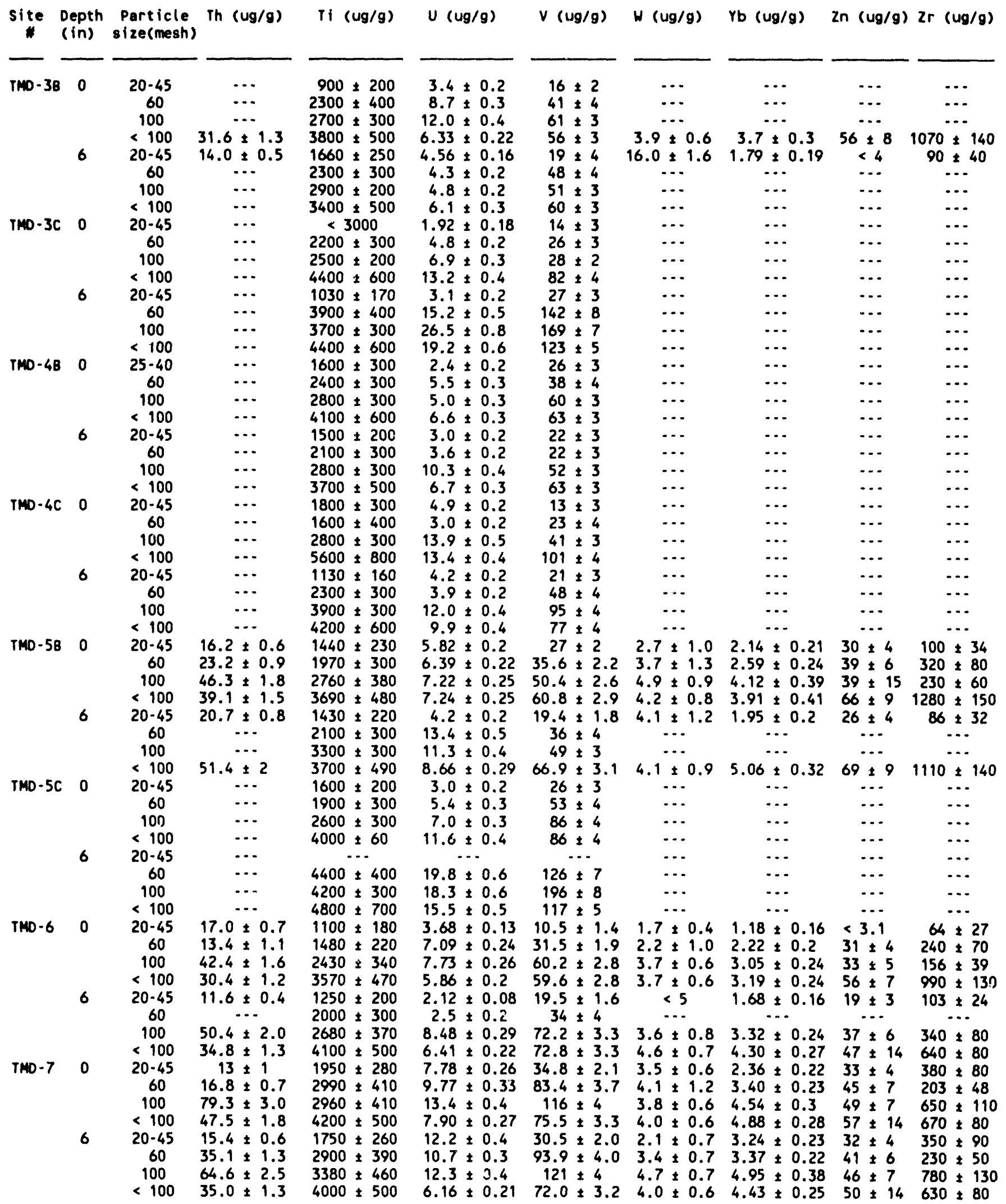




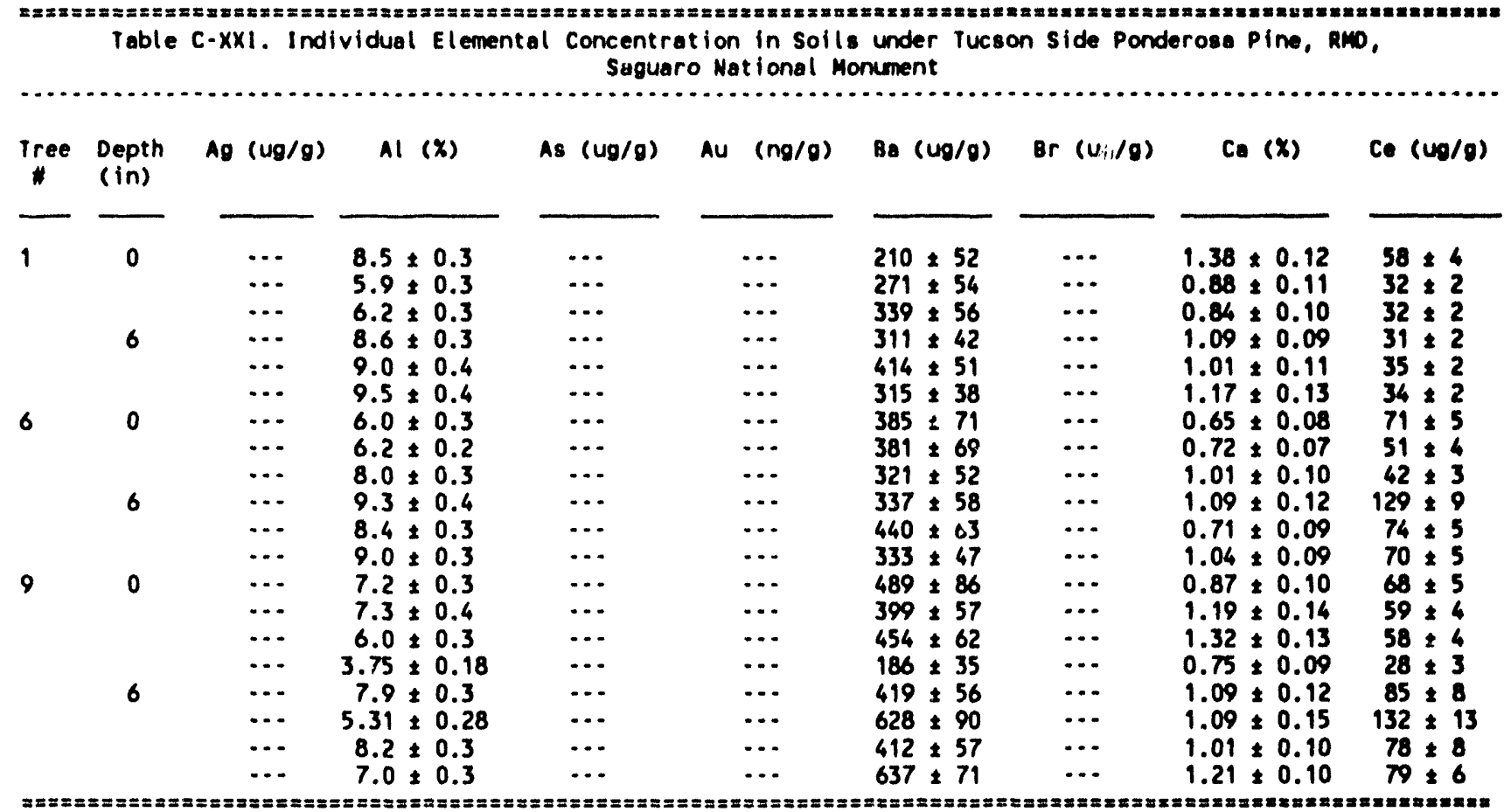

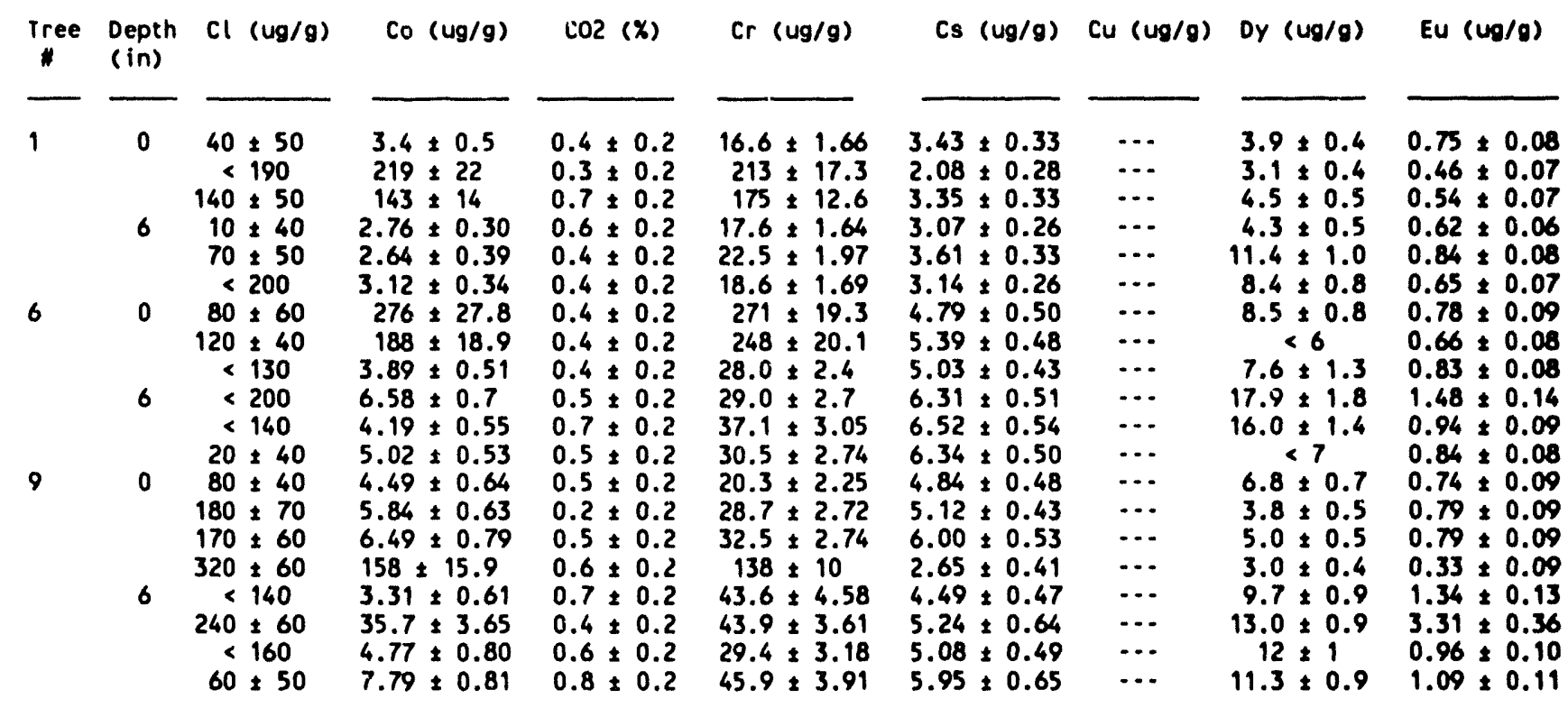




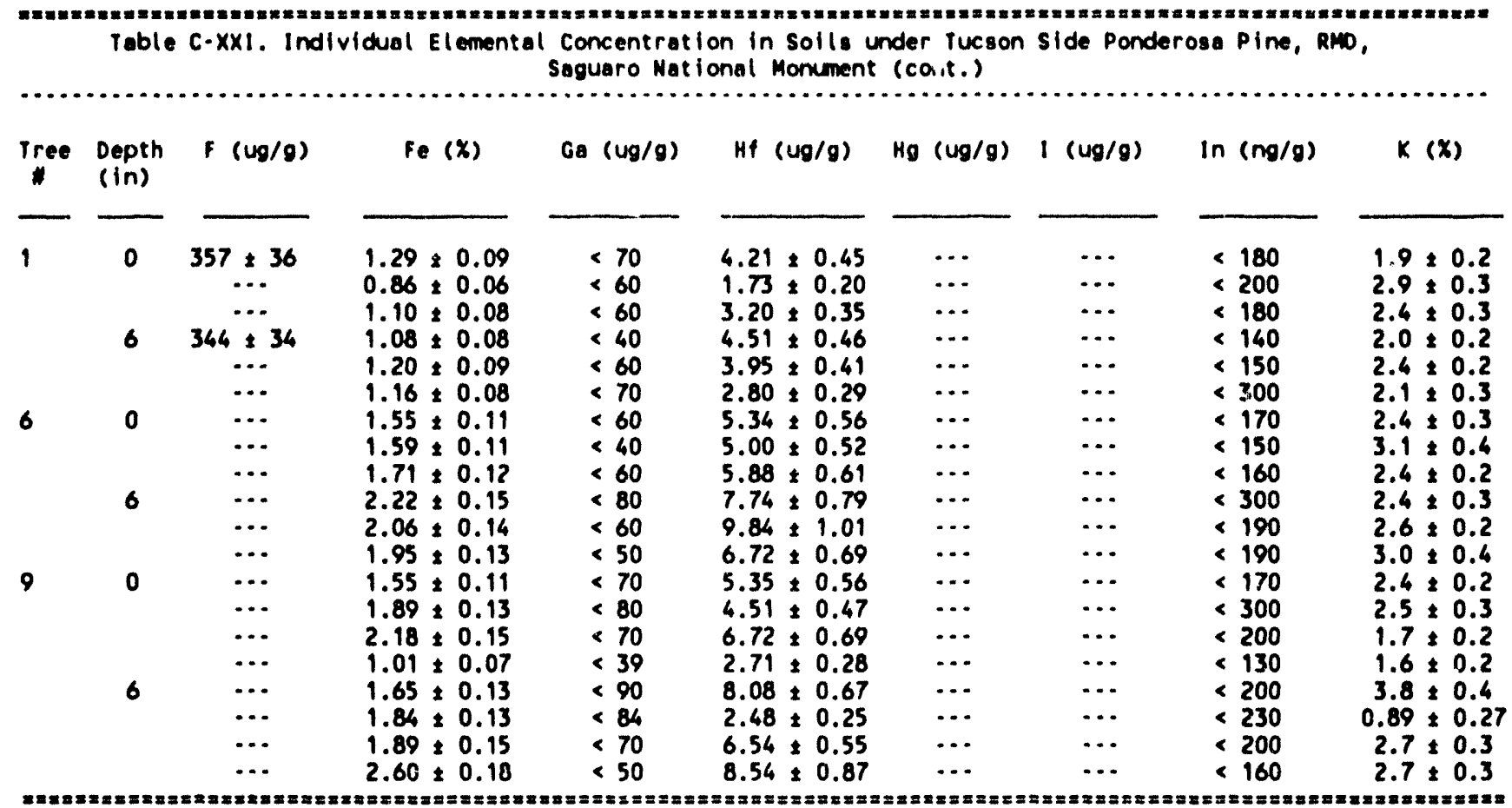

\begin{tabular}{|c|c|c|c|c|c|c|c|c|c|}
\hline $\begin{array}{c}\text { Tree } \\
\text { : }\end{array}$ & $\begin{array}{l}\text { Depth } \\
\text { (in) }\end{array}$ & La $(u g / g)$ & $\operatorname{Lu}(u g / g)$ & $M g(u g / g)$ & Mn (ug/g) & $\mathrm{Na}(u g / g)$ & Nd (ug/g) & pH (units) & Rb $(4 g / g)$ \\
\hline \multirow[t]{6}{*}{1} & 0 & $\cdots$ & $\ldots$ & $<5000$ & $1290 \div 60$ & $33100 \div 1100$ & $\cdots$ & $5.1 \pm 0.3$ & $122 \div 11$ \\
\hline & & $\cdots$ & $\cdots$ & $<6000$ & $840 \pm 40$ & $20200 \div 700$ & $\cdots$ & $\ldots$ & $131=13$ \\
\hline & & $\cdots$ & $\cdots$ & $<4000$ & $1030 \pm 50$ & $20600 \div 700$ & $\cdots$ & $\cdots$ & $137 \div 16$ \\
\hline & 6 & $\cdots$ & $\cdots$ & $<4000$ & $820 \div 40$ & $33300 \pm 1100$ & $\cdots$ & $5.4 \pm 0.3$ & $122 \div 10$ \\
\hline & & $\cdots$ & $\cdots$ & $<4000$ & $740 \div 30$ & $32000 \div 1100$ & $\cdots$ & $\cdots$ & $124 \div 11$ \\
\hline & & $\cdots$ & $\cdots$ & $<6000$ & $1200 \div 60$ & $35100 \div 1200$ & $\cdots$ & $\cdots$ & $130 \div 11$ \\
\hline \multirow[t]{6}{*}{6} & 0 & $\cdots$ & $\cdots$ & $<4000$ & $1210 \div 50$ & $14400 \div 500$ & $\cdots$ & $\cdots$ & $163+16$ \\
\hline & & $\cdots$ & $\cdots$ & $<3000$ & $1150 \pm 50$ & $15400 \pm 500$ & $\cdots$ & $\cdots$ & $172 \pm 16$ \\
\hline & & $\cdots$ & $\cdots$ & $<4000$ & $1320 \pm 60$ & $25900 \pm 900$ & $\cdots$ & $\cdots$ & $163 \pm 14$ \\
\hline & 6 & $\cdots$ & $\cdots$ & $<7000$ & $1800 \simeq 80$ & $24600 \pm 900$ & $\cdots$ & $\cdots$ & $161 \div 14$ \\
\hline & & $\cdots$ & $\cdots$ & $<4000$ & $1390 \pm 60$ & $21100 \div 700$ & $\cdots$ & $\cdots$ & $183 \pm 16$ \\
\hline & & $\cdots$ & $\cdots$ & $<4000$ & $2300 \div 110$ & $24600 \div 800$ & $\cdots$ & $\cdots$ & $184+16$ \\
\hline \multirow[t]{8}{*}{9} & 0 & $\cdots$ & $\cdots$ & $<4000$ & $1210 \pm 60$ & $20500 \div 700$ & $\cdots$ & $\cdots$ & $172 \div 16$ \\
\hline & & $\cdots$ & $\cdots$ & $<7000$ & $1360 \pm 70$ & $17100 \neq 700$ & $\cdots$ & $\cdots$ & $147 \pm 13$ \\
\hline & & $\cdots$ & $\cdots$ & $4500 \div 1500$ & $1120 \div 50$ & $14800 \div 500$ & $\cdots$ & $\cdots$ & $139 \div 13$ \\
\hline & & $\cdots$ & $\cdots$ & $5300 \div 1300$ & $407 \div 19$ & $8700 \div 300$ & $\cdots$ & $\cdots$ & $122 \div 16$ \\
\hline & 6 & $\cdots$ & $\cdots$ & $<5000$ & $3110 \div 140$ & $24100 \div 800$ & $\cdots$ & $\cdots$ & $192 \pm 20$ \\
\hline & & $\cdots$ & $\cdots$ & $7100 \div 1500$ & $1690 \div 80$ & $3550 \div 140$ & $\cdots$ & $\cdots$ & $83+12$ \\
\hline & & $\cdots$ & $\cdots$ & $<4000$ & $2110 \pm 90$ & $24600 \div 800$ & $\cdots$ & $\cdots$ & $194 \div 20$ \\
\hline & & $\cdots$ & $\cdots$ & $6400 \pm 1200$ & $1390 \pm 60$ & $19000 \div 600$ & $\cdots$ & $\cdots$ & $169 \div 18$ \\
\hline
\end{tabular}




\begin{tabular}{|c|c|c|c|c|c|c|c|c|c|c|}
\hline $\begin{array}{c}\text { Tree } \\
\|\end{array}$ & $\begin{array}{l}\text { Depth } \\
\text { (In) }\end{array}$ & Sb $(u g / g)$ & $\operatorname{sc}(u g / g)$ & Se $(u g / g)$ & si $(x)$ & $\mathrm{Sm}$ & $(40 / g)$ & $\operatorname{sr}(u g / g)$ & $\operatorname{Ta}(u g / g)$ & Tb $(u g / g)$ \\
\hline
\end{tabular}

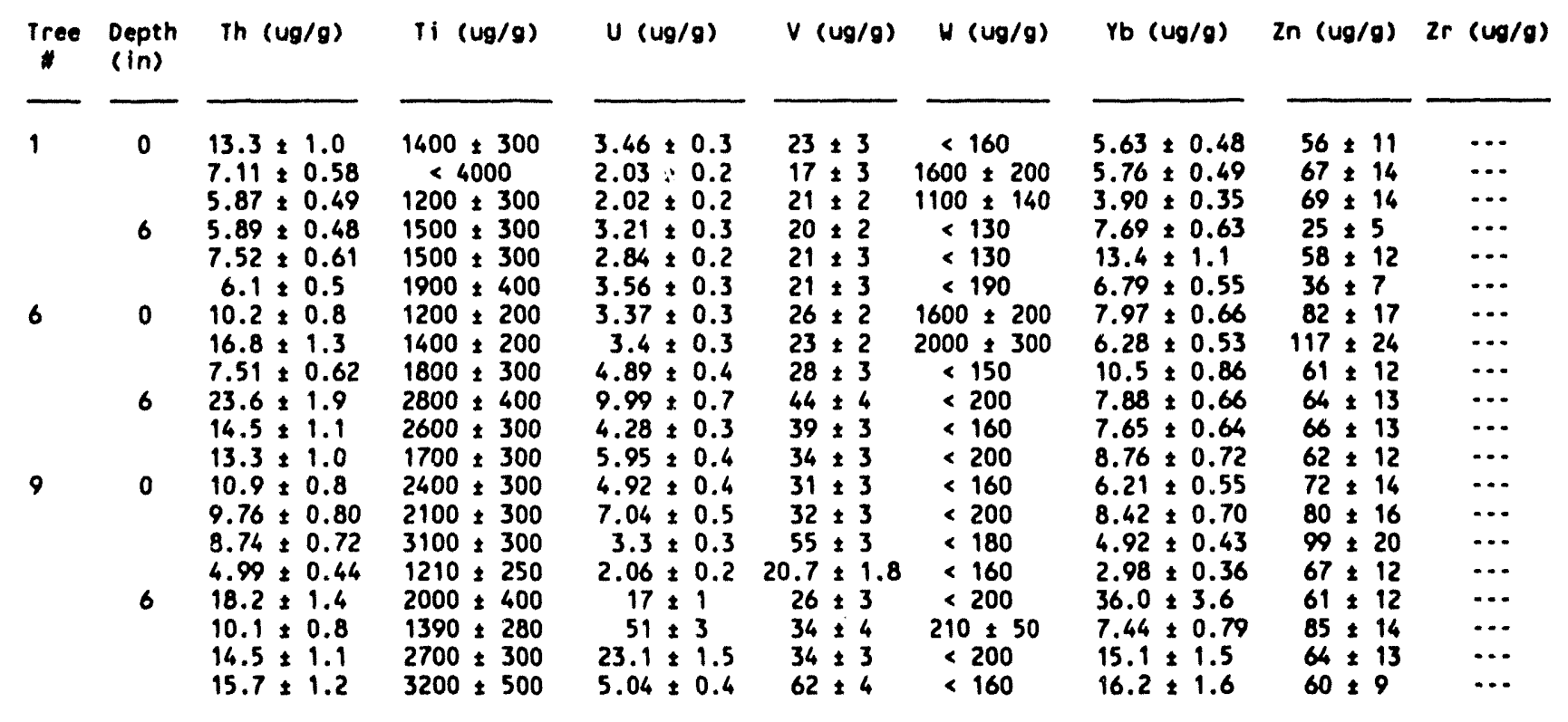

Note: Trees showing evidence of severe growth depression. 4, 6, 7, 8, 9.5 (4). 


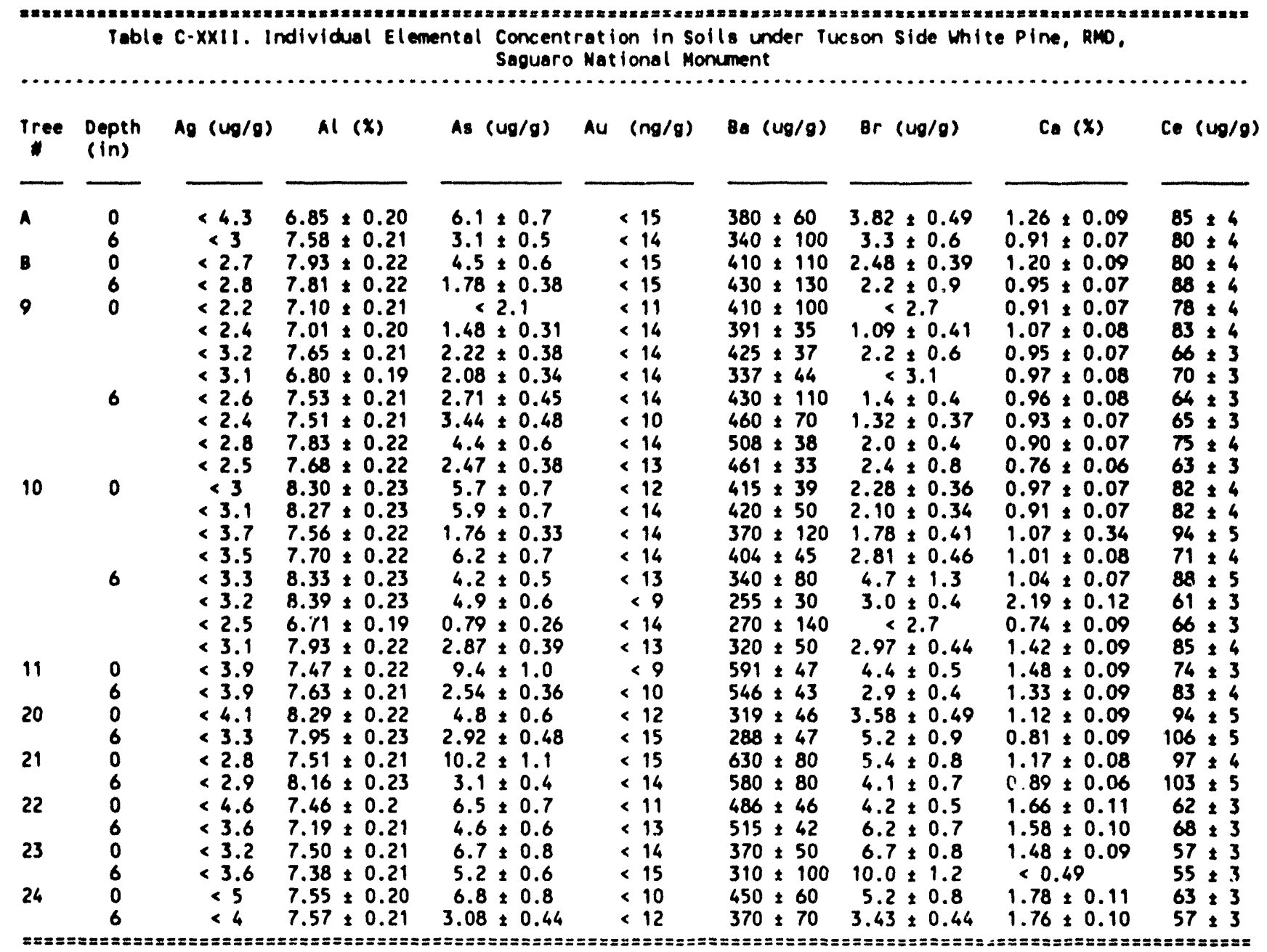

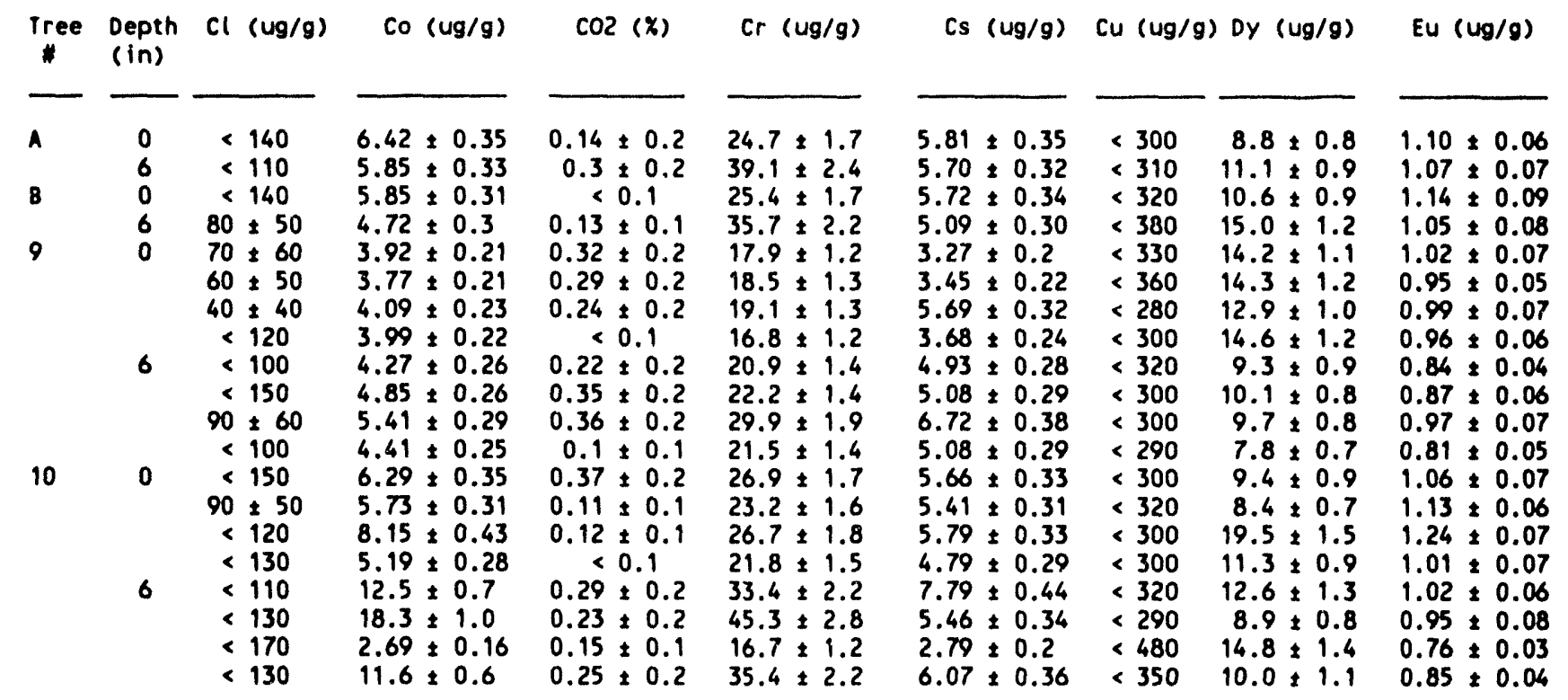


rable C-Xxil. Individual Elemental Concentration in Solls under rucson side White pine, Rino, Saguaro National Monument (cont.)

\begin{tabular}{|c|c|c|c|c|c|c|c|c|c|c|c|c|c|c|}
\hline Tree & $\begin{array}{l}\text { Depth } \\
\text { (in) }\end{array}$ & $\mathrm{Cl}$ & $(\log / \theta)$ & Co & (ug/g) & $\cos (x)$ & $\operatorname{cr}(40 / 0)$ & $\mathrm{Cs}$ & $(40 / 0)$ & $\mathrm{Cu}(u g / \theta)$ & Dy & $(\omega 0 / 0)$ & Eur & $(40 / 9)$ \\
\hline $\begin{array}{l}11 \\
20 \\
21 \\
? 2 \\
23 \\
24\end{array}$ & $\begin{array}{l}0 \\
6 \\
0 \\
6 \\
0 \\
6 \\
0 \\
6 \\
0 \\
6 \\
0 \\
6\end{array}$ & $\begin{array}{r}100 \\
100 \\
5 \\
40 \\
150 \\
6 \\
60 \\
160 \\
100 \\
<\end{array}$ & $\begin{array}{l}+50 \\
140 \\
160 \\
190 \\
130 \\
+50 \\
150 \\
120 \\
150 \\
150 \\
150 \\
100\end{array}$ & $\begin{array}{l}17.3 \\
20.6 \\
5.67 \\
5.51 \\
8.58 \\
8.19 \\
12.8 \\
12.7 \\
12.9 \\
11.3 \\
17.4 \\
18.5\end{array}$ & 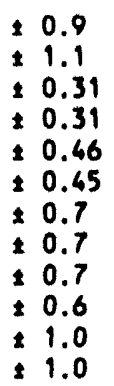 & $\begin{array}{r:r}0.16: 0.2 \\
0.43: 0.2 \\
0.45: 0.2 \\
0.2: 0.2 \\
0.51: 0.2 \\
0.07: 0.2 \\
0.22: 0.2 \\
0.18: 0.1 \\
0.25: 0.2 \\
0.4: 0.2 \\
0.41: 0.2 \\
0.53: 0.2\end{array}$ & $\begin{array}{l}56.2: 3.5 \\
57.1: 3.5 \\
20.5: 1.5 \\
27.1: 1.8 \\
40.7: 2.5 \\
44.8: 2.7 \\
33.4: 2.2 \\
48.1: 3.0 \\
29.3: 1.9 \\
38.0: 2.4 \\
31.2: 2.1 \\
34.7: 2.3\end{array}$ & $\begin{array}{l}12.8 \\
13.8 \\
5.18 \\
4.55 \\
8.03 \\
8.35 \\
7.60 \\
7.46 \\
7.70 \\
7.42 \\
8.9 \\
8.24\end{array}$ & $\begin{array}{ll}1 & 0.7 \\
1 & 0.8 \\
1 & 0.32 \\
1 & 0.3 \\
1 & 0.47 \\
1 & 0.47 \\
1 & 0.44 \\
1 & 0.44 \\
1 & 0.44 \\
1 & 0.45 \\
1 & 0.5 \\
1 & 0.47\end{array}$ & $\begin{array}{l}290 \\
<300 \\
\times 420 \\
\times \quad 500 \\
<280 \\
<260 \\
<300 \\
<370 \\
<280 \\
<300 \\
<300 \\
<290\end{array}$ & $\begin{array}{l}6.94 \\
5.15 \\
19.0 \\
17.4 \\
8.5 \\
7.5 \\
6.3 \\
4.4 \\
6.3 \\
6.7 \\
5.6 \\
5.16\end{array}$ & $\begin{array}{l}10.48 \\
10.46 \\
1.8 \\
1.5 \\
10.7 \\
10.7 \\
10.7 \\
10.6 \\
10.6 \\
10.7 \\
10.5 \\
10.68\end{array}$ & $\begin{array}{l}1.04 \\
1.18 \\
1.38 \\
1.30 \\
1.36 \\
1.46 \\
1.04 \\
1.08 \\
0.96 \\
0.86 \\
1.03 \\
0.99\end{array}$ & 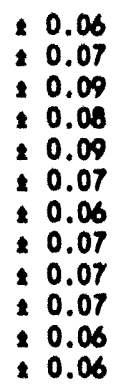 \\
\hline
\end{tabular}

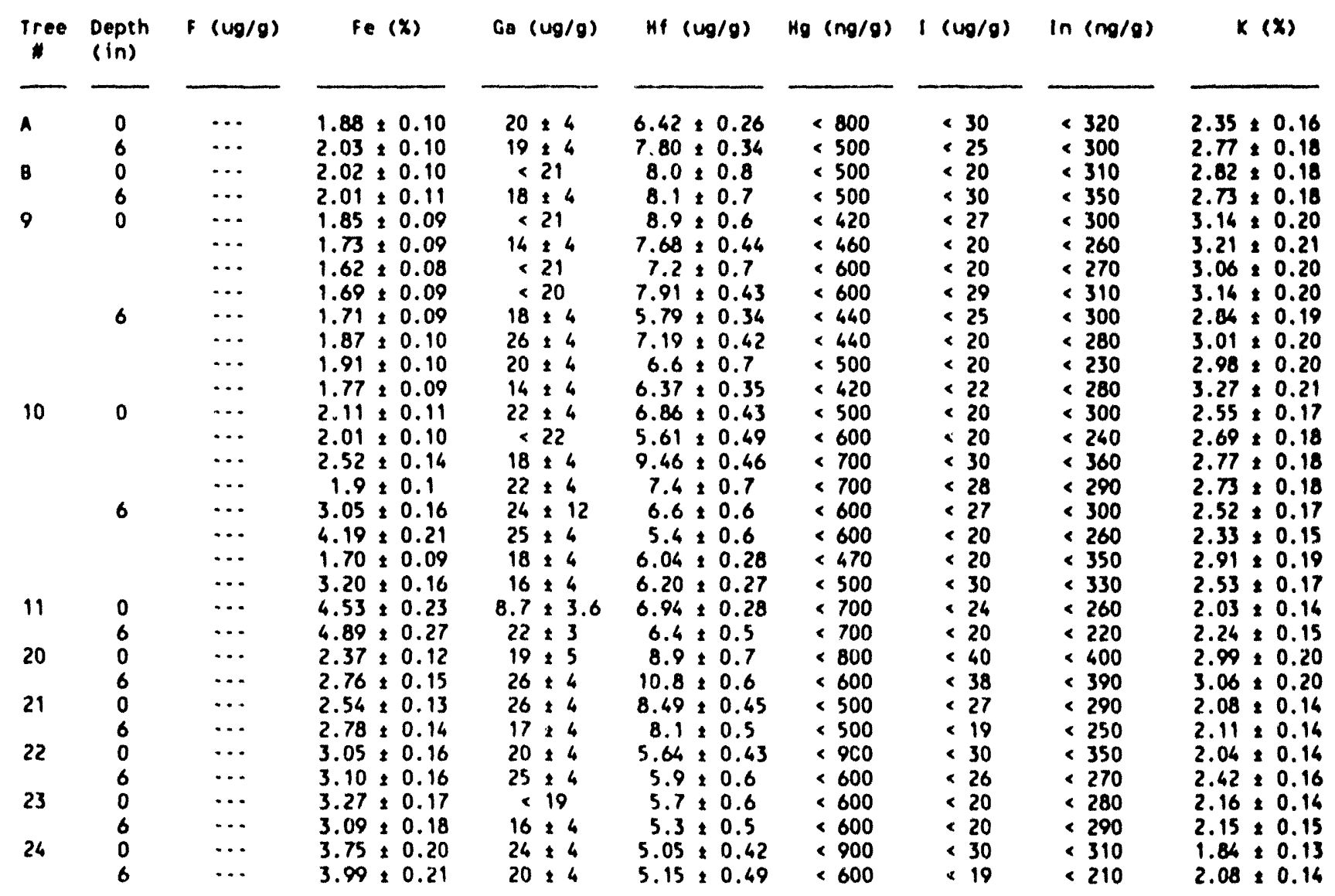




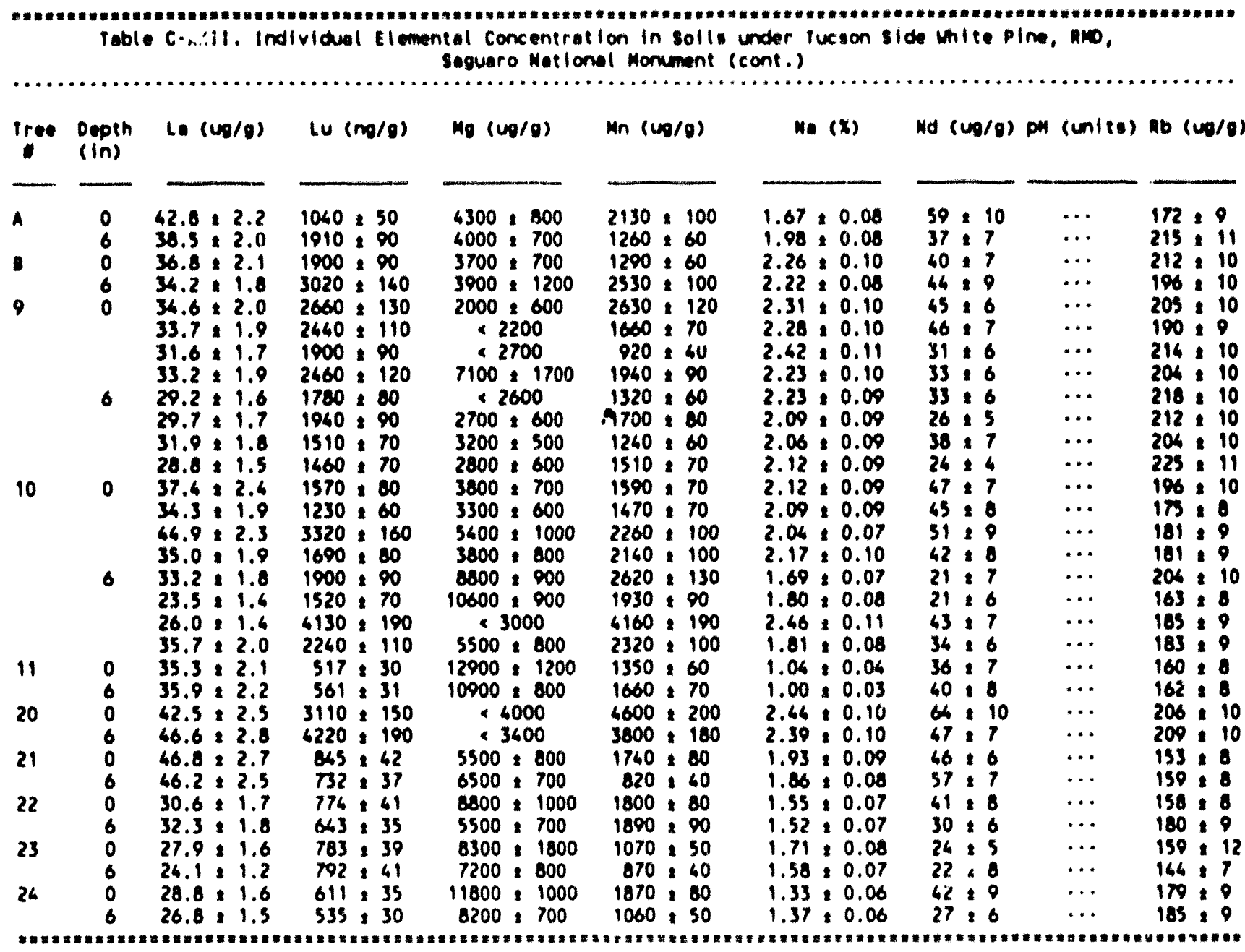

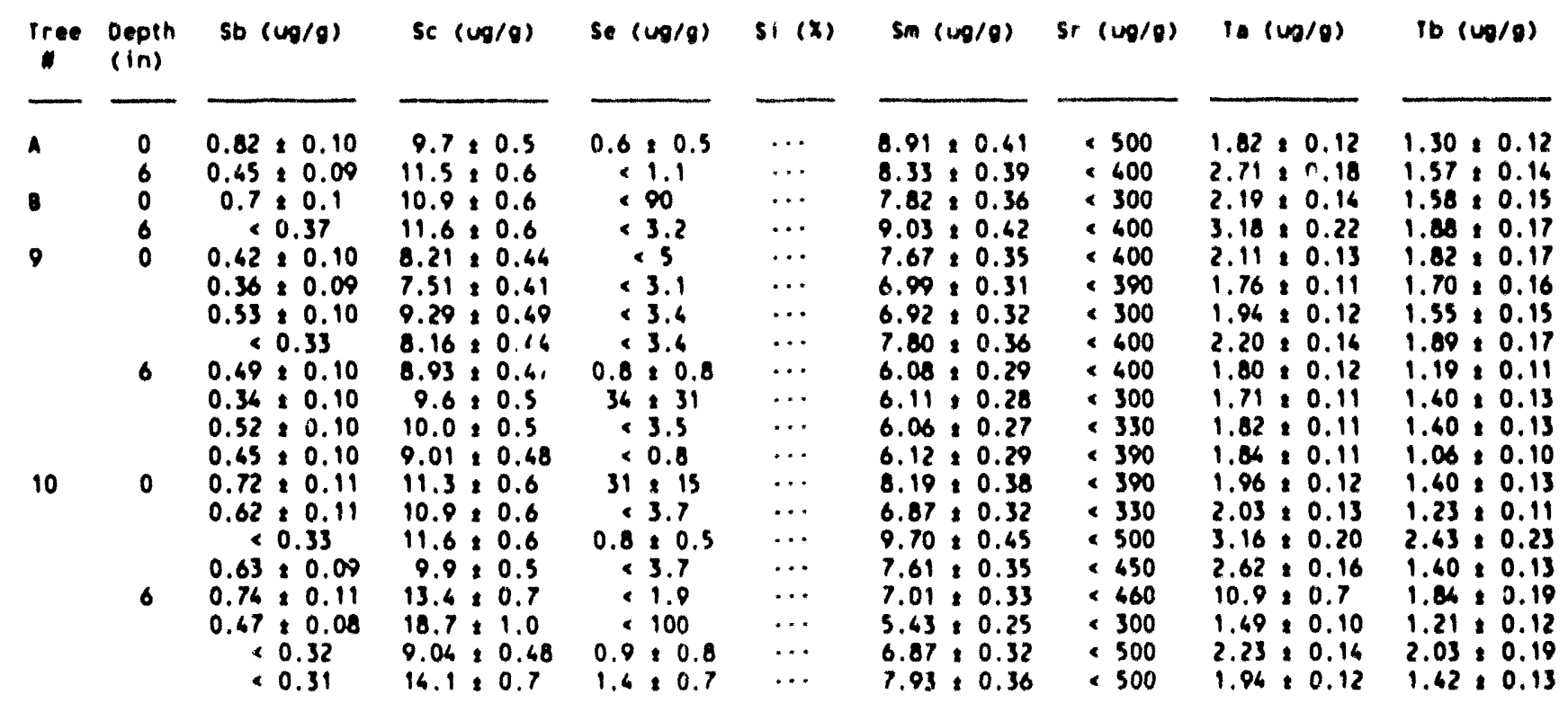




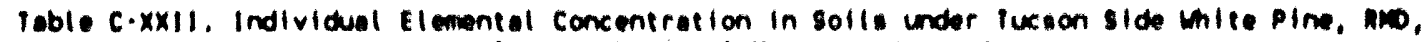
seguare vational Momment (cont.)

\begin{tabular}{|c|c|c|c|c|c|c|c|c|c|}
\hline iree & $\begin{array}{l}\text { Depth } \\
\text { (In) }\end{array}$ & $9 b(w / 0)$ & se $(\omega \rho / g)$ & se $(\omega 0 / 0)$ & $31(x)$ & $\operatorname{sen}\left(\log _{0} / 0\right)$ & $\operatorname{sr}(\omega \theta / \theta)$ & te $(\omega 0 / 0)$ & If $(\omega / \theta)$ \\
\hline $\begin{array}{l}20 \\
21 \\
22 \\
23\end{array}$ & $\begin{array}{l}0 \\
6 \\
0 \\
6 \\
0 \\
6 \\
0 \\
6 \\
0 \\
6 \\
0 \\
6\end{array}$ & $\begin{array}{r:l}0.91 & 0.10 \\
0.31 & 0.00 \\
: & 0.37 \\
0.07 & 0.36 \\
0.97 & 0.11 \\
0.35 & 0.11 \\
0.82 & 0.11 \\
0.61 & 0.00 \\
0.75 & 0.10 \\
0.50 & 0.11 \\
0.63 & 0.10 \\
0.45 & 0.08\end{array}$ & $\begin{array}{r:l}16.5 & 0.9 \\
18.2 & 1.0 \\
12.1 & 0.7 \\
12.9 & 0.7 \\
9.6 & 0.5 \\
10.1 & 0.5 \\
12.0 & 0.6 \\
11.9 & 0.6 \\
12.8 & 0.7 \\
11.8 & 0.6 \\
16.7 & 0.8 \\
15.6 & 0.8\end{array}$ & $\begin{array}{r:l}93 & 20 \\
1.2 & 0.3 \\
1.2 & 0.7 \\
2.4 & 1.3 \\
: 100 \\
3.3 \\
1.7 & 0.7 \\
0.8 & 0.4 \\
: 100 \\
1.9 & 0.8 \\
1 & 0.8\end{array}$ & $\begin{array}{l}\ldots \\
\ldots \\
\ldots \\
\ldots \\
\ldots \\
\ldots \\
\ldots \\
\ldots \\
\ldots \\
\ldots\end{array}$ & $\begin{array}{l:l}5.84: 0.26 \\
5.91: 0.27 \\
10: 0.48 \\
10.5: 0.47 \\
6.43: 0.39 \\
8.18: 0.38 \\
5.67: 0.26 \\
3.61: 0.26 \\
6.93: 0.22 \\
6.67: 0.22 \\
5.18: 0.23 \\
4.70: 0.21\end{array}$ & $\begin{array}{l}300 \\
360 \\
700 \\
600 \\
400 \\
300 \\
300 \\
420 \\
4300 \\
300 \\
450 \\
310\end{array}$ & $\begin{array}{l:l}1.18: 0.10 \\
1.26: 0.12 \\
2.71: 0.17 \\
5.32: 0.23 \\
1.33: 0.09 \\
1.21: 0.09 \\
1.33: 0.10 \\
1.26: 0.09 \\
1.53: 0.10 \\
1.30: 0.13 \\
1.39: 0.10 \\
1.40: 0.11\end{array}$ & $\begin{array}{l:l}0.86 & 0.00 \\
0.78 & 0.00 \\
2.64 & 0.25 \\
2.69 & 0.23 \\
1.18 & 0.11 \\
1.11 & 0.10 \\
0.85 & 0.10 \\
0.83 & 0.08 \\
0.87 & 0.09 \\
0.80 & 0.06 \\
0.91 & 0.10 \\
0.65 & 0.07\end{array}$ \\
\hline
\end{tabular}

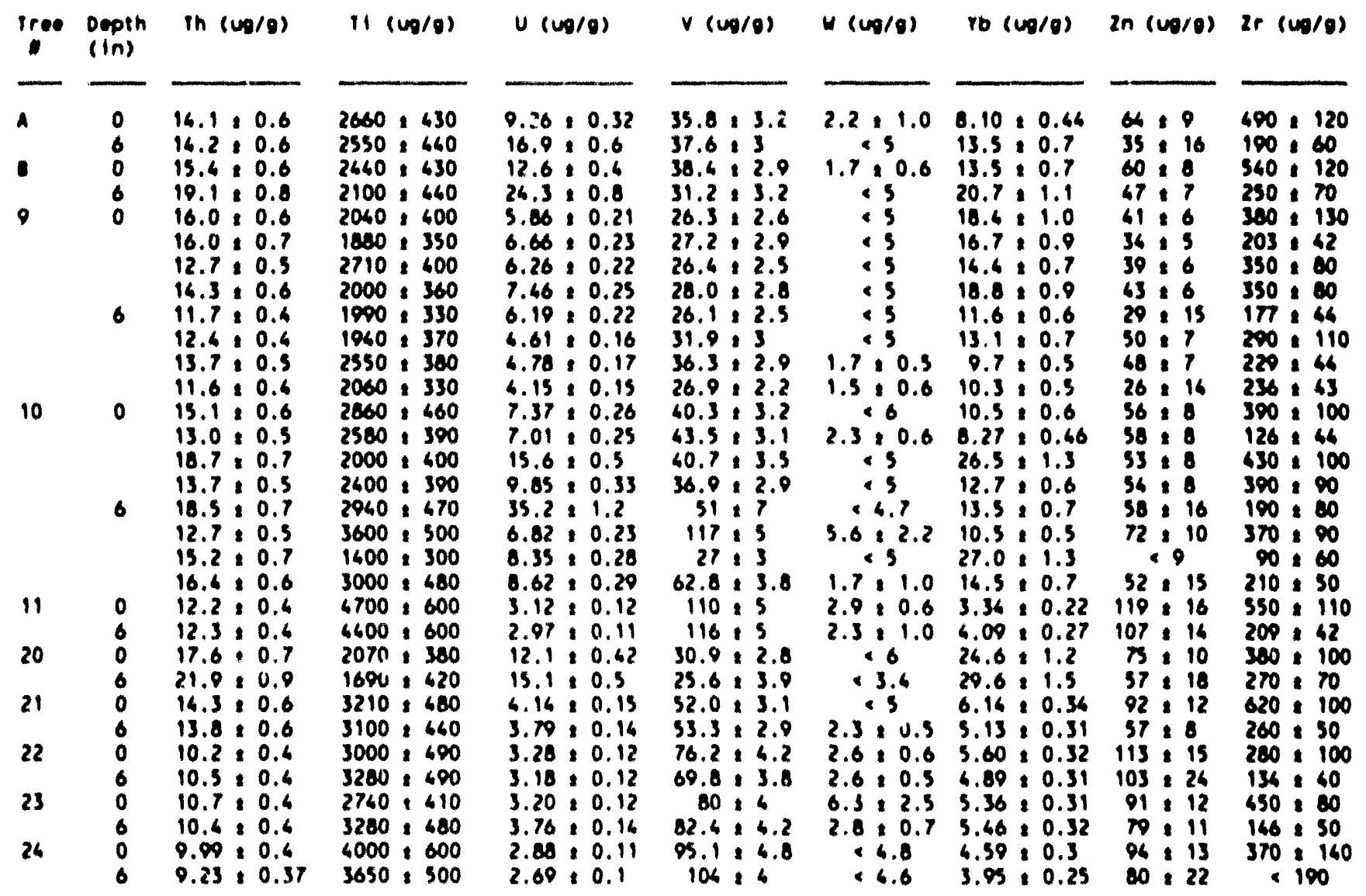




\begin{tabular}{|c|c|c|c|c|c|c|c|c|c|}
\hline Ires & $\begin{array}{l}\text { oppth } \\
(|n\rangle\end{array}$ & As $(u, \theta)$ & Al (x) & $A=(w / a)$ & Au $(n g / \theta)$ & ot $(w / g)$ & $\operatorname{le}(\omega, \theta)$ & $c e(x)$ & $\operatorname{co}(u / \theta)$ \\
\hline
\end{tabular}

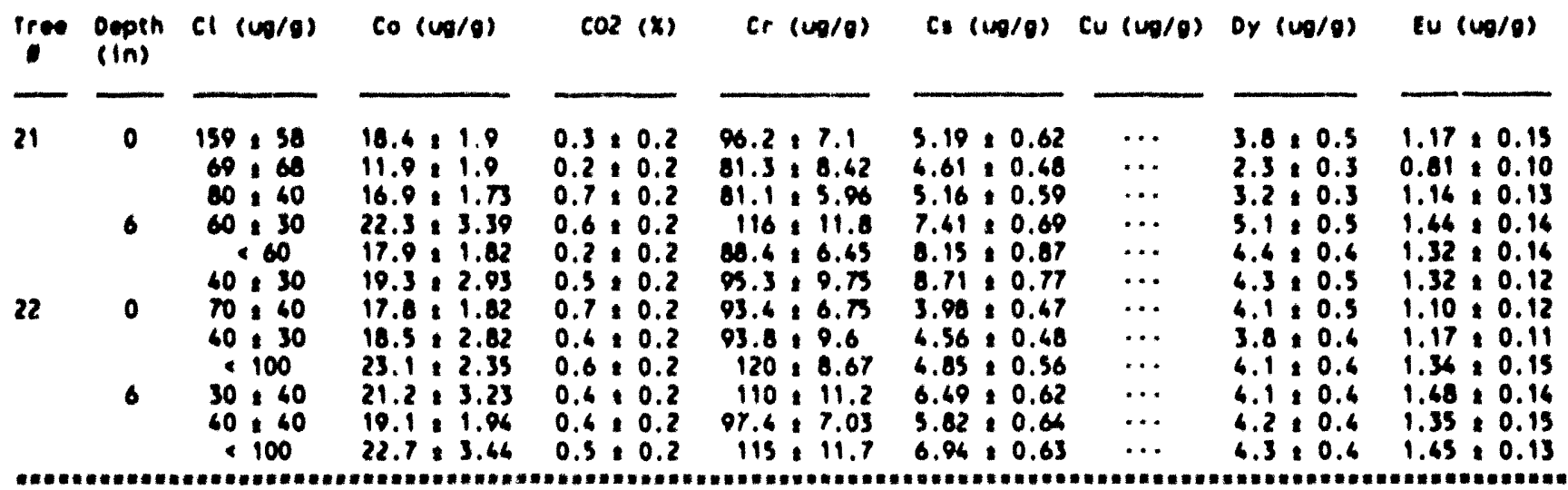

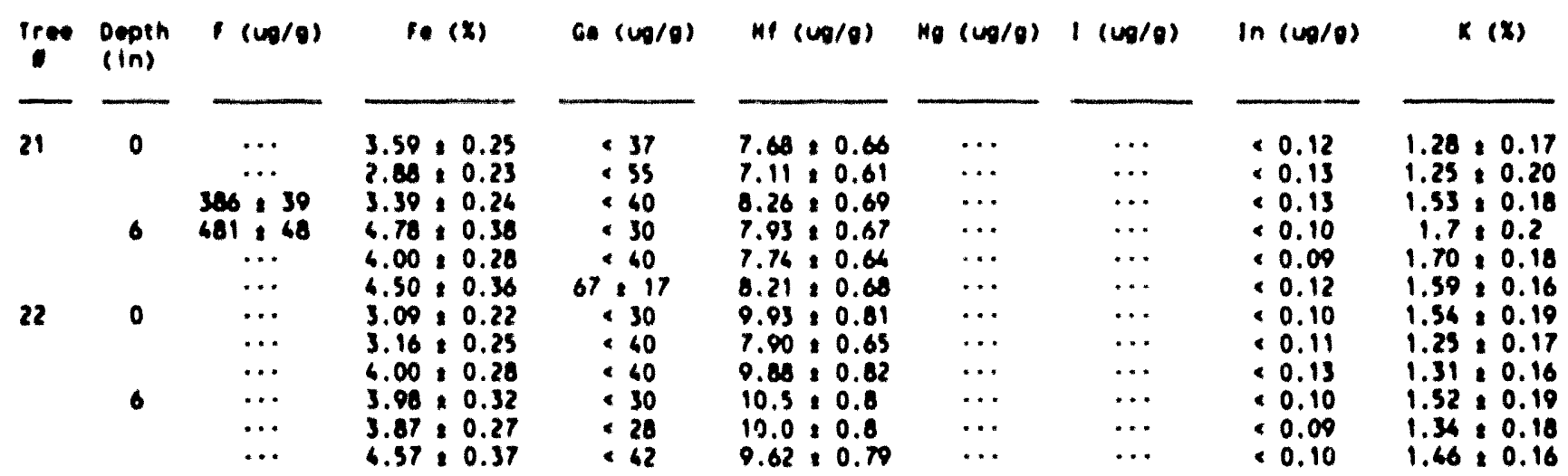


leble c-xxill. Individual Elemental Concentration in soils under Ponderose Pine, Wal nut Canyon Mational Morument, Az (cont.)

\begin{tabular}{|c|c|c|c|c|c|c|c|c|c|c|}
\hline Iree & $\begin{array}{l}\text { Depth } \\
\text { (In) }\end{array}$ & (1. $(\omega g / \theta)$ & $\operatorname{lu}(u g / g)$ & $\operatorname{Mg}(x)$ & $\operatorname{Mn}(\omega 0 / \theta)$ & No & $(x)$ & Nd $(u p / \theta)$ & pH (untes) & $0\left(v_{\theta} / \theta\right)$ \\
\hline \multirow[t]{6}{*}{21} & 0 & $\ldots$ & $\ldots$ & \multirow{12}{*}{$\begin{array}{l}0.10 \\
0.15 \\
0.13 \\
0.10 \\
0.09 \\
0.11 \\
0.09 \\
0.12 \\
0.11 \\
0.09 \\
0.11 \\
0.11\end{array}$} & \multirow{12}{*}{$\begin{array}{l:l}622 & 28 \\
590 & 27 \\
580 & 30 \\
680 & 30 \\
560 & 30 \\
580 & 30 \\
610 & 30 \\
660 & 30 \\
690 & 30 \\
630 & 30 \\
590 & 27 \\
649 & 30\end{array}$} & \multirow{6}{*}{$\begin{array}{l}0.45 \\
0.42 \\
0.42 \\
0.34 \\
0.29 \\
0.29\end{array}$} & \multirow{6}{*}{$\begin{array}{l}0.02 \\
0.02 \\
0.02 \\
0.01 \\
0.01 \\
0.01\end{array}$} & $\ldots$ & $\cdots$ & \multirow{12}{*}{ 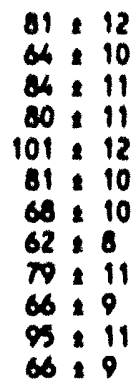 } \\
\hline & & $\ldots$ & $\ldots$ & & & & & $\ldots$ & $\ldots$ & \\
\hline & & $\ldots$ & $\ldots$ & & & & & $\ldots$ & $6.5: 0.3$ & \\
\hline & 6 & $\cdots$ & $\cdots$ & & & & & $\ldots$ & $6.2: 0.3$ & \\
\hline & & $\cdots$ & $\cdots$ & & & & & $\cdots$ & ... & \\
\hline & & $\ldots$ & $\ldots$ & & & & & $\ldots$ & $\ldots$ & \\
\hline \multirow[t]{6}{*}{22} & 0 & $\cdots$ & $\ldots$ & & & $0.59=$ & 0.02 & $\cdots$ & $\cdots$ & \\
\hline & & $\cdots$ & $\ldots$ & & & 0.53 & 0.02 & $\cdots$ & $\cdots$ & \\
\hline & & $\cdots$ & $\cdots$ & & & 0.59 & 0.02 & $\cdots$ & $\ldots$ & \\
\hline & 6 & $\cdots$ & $\cdots$ & & & $0.51=$ & 0.02 & $\cdots$ & $\cdots$ & \\
\hline & & $\cdots$ & $\cdots$ & & & 0.48 & 0.02 & $\cdots$ & $\cdots$ & \\
\hline & & $\cdots$ & $\ldots$ & & & 0.50 & 0.02 & $\cdots$ & $\cdots$ & \\
\hline
\end{tabular}

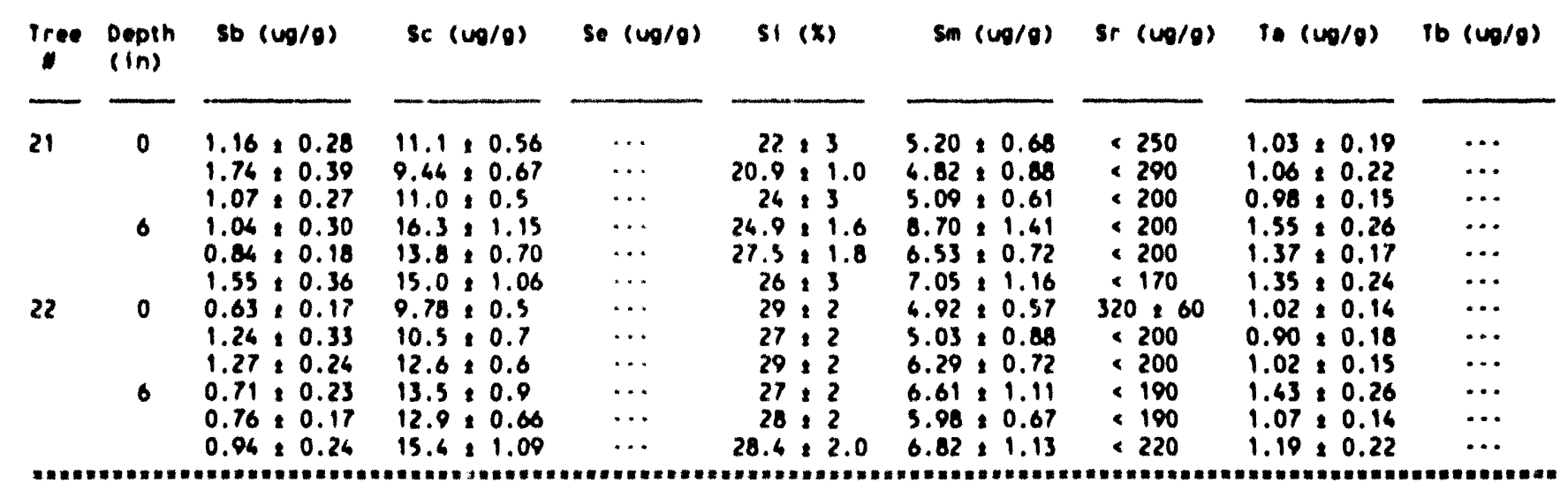

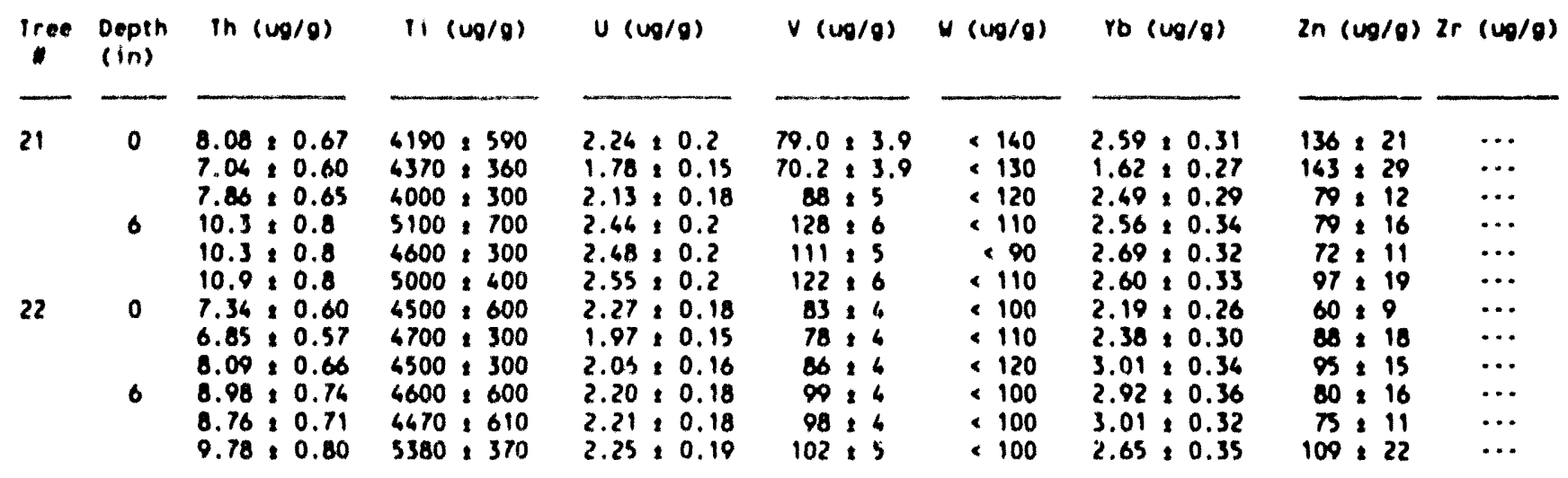



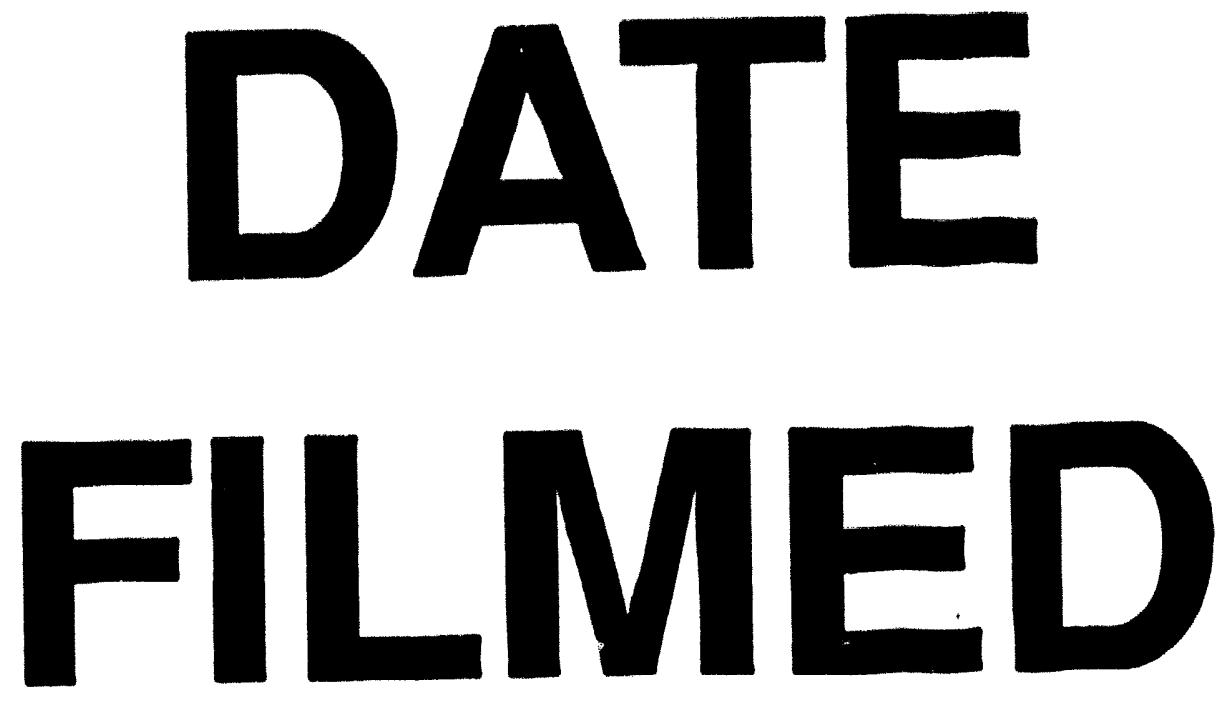

$10 / 15 / 93$
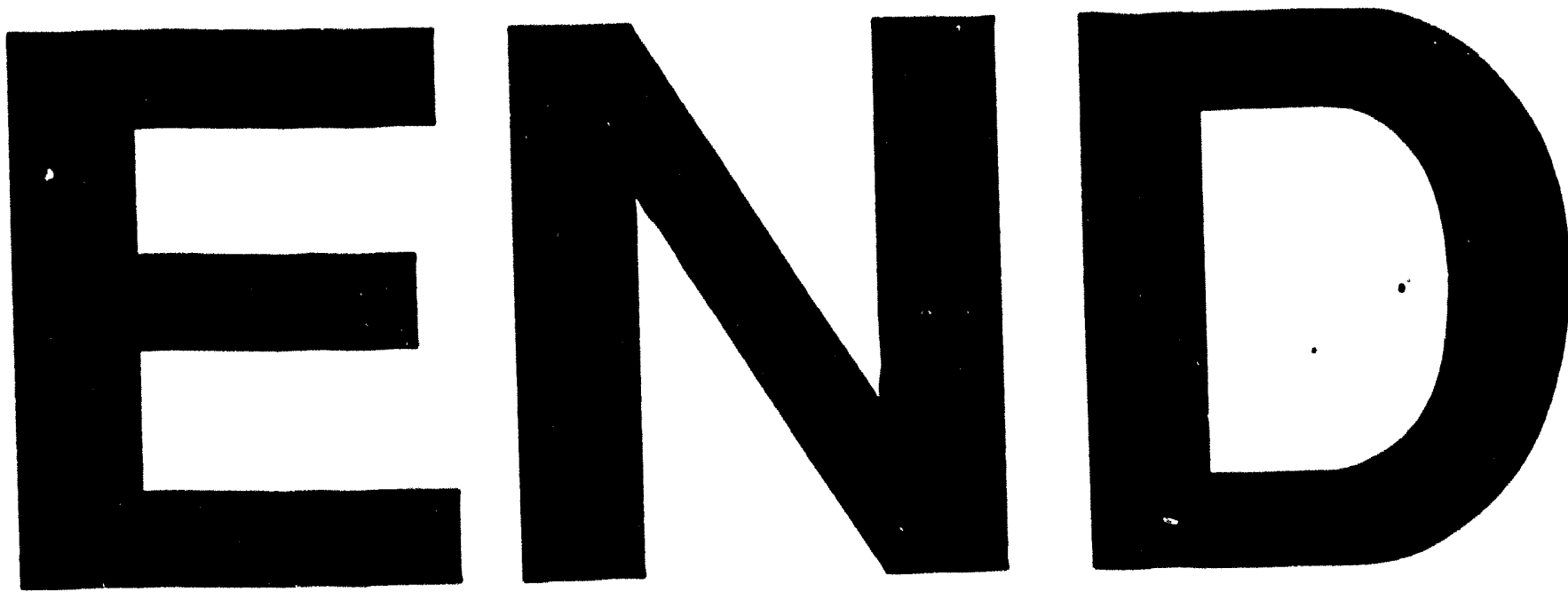